\title{
Dielectric Resonator Antenna Arrays in the EHF Band
}

\author{
by Mark Wyville
}

A thesis submitted to the Department of Electronics in conformity with the requirements for the degree of Master of Applied Science

\author{
Ottawa-Carleton Institute for Electrical Engineering \\ Department of Electronics \\ Carleton University \\ Ottawa, Ontario
}

Copyright (C2005, Mark Wyville 


$\begin{array}{ll}\begin{array}{l}\text { Library and } \\ \text { Archives Canada }\end{array} & \begin{array}{l}\text { Bibliothèque et } \\ \text { Archives Canada }\end{array} \\ \begin{array}{l}\text { Published Heritage } \\ \text { Branch }\end{array} & \begin{array}{l}\text { Direction du } \\ \text { Patrimoine de l'édition }\end{array} \\ \begin{array}{l}\text { 395 Wellington Street } \\ \text { Ottawa ON K1A ON4 }\end{array} & \begin{array}{l}\text { 395, rue Wellington } \\ \text { Ottawa ON K1A ON4 } \\ \text { Canada }\end{array}\end{array}$

Your file Votre référence

ISBN: 0-494-13453-4

Ourfile Notre référence

ISBN: 0-494-13453-4

NOTICE:

The author has granted a nonexclusive license allowing Library and Archives Canada to reproduce, publish, archive, preserve, conserve, communicate to the public by telecommunication or on the Internet, loan, distribute and sell theses worldwide, for commercial or noncommercial purposes, in microform, paper, electronic and/or any other formats.

The author retains copyright ownership and moral rights in this thesis. Neither the thesis nor substantial extracts from it may be printed or otherwise reproduced without the author's permission.
AVIS:

L'auteur a accordé une licence non exclusive permettant à la Bibliothèque et Archives Canada de reproduire, publier, archiver, sauvegarder, conserver, transmettre au public par télécommunication ou par l'Internet, prêter, distribuer et vendre des thèses partout dans le monde, à des fins commerciales ou autres, sur support microforme, papier, électronique et/ou autres formats.

L'auteur conserve la propriété du droit d'auteur et des droits moraux qui protège cette thèse. $\mathrm{Ni}$ la thèse ni des extraits substantiels de celle-ci ne doivent être imprimés ou autrement reproduits sans son autorisation.
In compliance with the Canadian

Privacy Act some supporting forms may have been removed from this thesis.

While these forms may be included in the document page count, their removal does not represent any loss of content from the thesis.
Conformément à la loi canadienne sur la protection de la vie privée, quelques formulaires secondaires ont été enlevés de cette thèse.

Bien que ces formulaires aient inclus dans la pagination, il n'y aura aucun contenu manquant.

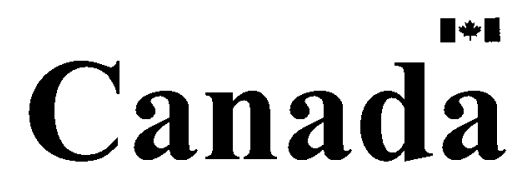




\begin{abstract}
Dielectric resonator antennas (DRA) are efficient radiators at high frequencies, however this desirable quality has generally been overshadowed by feed losses when used in an array. The goal of this research was to derive design guidelines for an array of DRA elements fed in series by a dielectric image guide (DIG). The DIG was chosen for its high frequency low-loss properties and the field configuration of its fundamental mode. The amplitude control was achieved by varying the distances between the DIG and the DRA's.
\end{abstract}

Coupled mode theory was used to approximate the coupling between the DIG and DRA's. This method was adequate for cases where the DIG supported a loosely bound fundamental mode. Several arrays with specific amplitude distributions were designed to validate the design guidelines.

A modification was introduced for DRA's to short out undesired radiating modes. Also, a series-fed array was designed with reduced frequency scanning. 


\section{Acknowledgements}

The commitments made by my supervisors, Aldo Petosa and Jim Wight, were greatly appreciated. Their supervision guided my work and immersed me in a research environment.

I wish to acknowledge the members of the antenna group at CRC for their insight ranging from practical issues to theoretical concepts to views on life during coffee breaks.

This work was made possible by financial contributions from NSERC, Nortel Networks, the Department of Graduate Studies and the Department of Electronics of Carleton University. 


\section{Contents}

1 Introduction 1

1.1 EHF Band . . . . . . . . . . . . . . . . . 3

1.1.1 Types of Antennas ............... 4

1.2 DRA Arrays . . . . . . . . . . . . . . . . 4

2 The Dielectric Resonator Antenna $\quad 7$

2.1 DRA Survey . . . . . . . . . . . . . . 7

2.1 .1 Survey Results . . . . . . . . . . . . . 10

2.2 Theory of Rectangular DRA's . . . . . . . . . . . 11

2.2.1 Resonant Frequency . . . . . . . . . . . . . 11

2.2 .2 Radiation ................... 13

2.2 .3 Mutual Coupling . . . . . . . . . . . . . 14

3 The Feed Network $\quad 16$

3.1 Transmission Line Survey . . . . . . . . . . . . . 16

3.1.1 Survey Results ................ 20

3.2 Characterization of the DIG . . . . . . . . . 21 
3.2.1 Characteristic Parameter . . . . . . . . . . 23

3.2 .2 Material Losses . . . . . . . . . . . . . . 26

3.2.3 Results for Specific DIG's . . . . . . . . . . . 31

3.3 Empire . . . . . . . . . . . . . . . . 32

3.4 Surface-Wave Launcher for the DIG . . . . . . . . . . . . . 36

3.4 .1 Launcher Survey . . . . . . . . . . . . . 37

3.4.2 Results for Specific Launchers . . . . . . . . . . . . 43

4 The DRA Array Fed by the DIG 47

4.1 The History of DIG Fed Arrays . . . . . . . . . . . . . 48

4.2 Coupling Between the DIG and the DRA - Analytical . . . . 49

4.2.1 Equivalent Circuit Model . . . . . . . . . . . . 50

4.2.2 Coupled Mode Theory . . . . . . . . . . . . 51

4.3 Coupling Between the DIG and the DRA - Simulation . . . 62

4.3 .1 Test $\operatorname{Setup} \ldots \ldots \ldots \ldots 6 . \ldots \ldots$

4.3 .2 Results ................... 70

4.4 Specific Amplitude Distributions . . . . . . . . . . . . 72

4.4.1 DRA Array with Uniform Amplitude

Distribution ............... 76

4.4.2 DRA Array with Chebyshev Amplitude

Distribution . . . . . . . . . . . . 79

4.4.3 DRA Array with Taylor Amplitude

Distribution ................. 82

4.5 The DRA Array Fed by Microstrip . . . . . . . . . . . 86 
4.5.1 Preliminary Design Considerations . . . . . . . . 87

4.5.2 Coupling Between Microstrip and the DRA Simulation . . . . . . . . . . . . . . . 91

4.5.3 Comparison to DIG Excited DRA Array . . . . . . . . 93

5 Array Performance Improvements $\quad 97$

5.1 Maximizing DRA Gain at $\theta=0^{\circ} \ldots \ldots . \ldots 98$

5.1.1 Parasitic DRA's . . . . . . . . . . . . . . 98

5.1 .2 Staggered DRA's ............... 101

5.1.3 Different Cross Sections Between DRA and DIG . . . 102

5.2 Reduction of Cross-Polarization . . . . . . . . . . 105

5.3 Reduction of Frequency Scanning . . . . . . . . . . 108

6 Conclusion $\quad 119$

A Mode-Matching Derivation - Two Parallel DIG's 122 


\section{List of Tables}

3.1 Design parameters for the DIG's . . . . . . . . . . 32

3.2 Transition losses at $30 \mathrm{GHz} \ldots \ldots . \ldots . \ldots 45$

4.1 Reflection due to presence of DRA at $30 \mathrm{GHz} \ldots \ldots 6$

4.2 Summary of losses for a single DRA for various cases . . . . 71

4.3 8-element array with uniform amplitude distribution . . . . 77

4.4 8-element array with Chebyshev amplitude distribution . . . . 80

4.5 16-element array with Taylor amplitude distribution . . . . . 85

4.6 Microstrip line dimensions . . . . . . . . . . . . . . 88

4.7 Summary of losses for a single DRA for various cases . . . . . 92

5.1 DRA parameters for array with reduced frequency scanning . 115

vii 


\section{List of Figures}

1.1 Layout of two DRA arrays (metallized objects are shaded) . . 3

1.2 Common antennas for EHF applications . . . . . . . . . 5

1.3 Directivity of array factors in array plane . . . . . . . . . . 6

2.1 Types of DRA's considered . . . . . . . . . . . . 8

2.2 Rectangular DRA with dimensions . . . . . . . . . . 11

2.3 Ideal normalized radiation pattern for $T E_{\delta 11}^{x}$ mode . . . . . 13

2.4 Two 2-element arrays showing physical gap between elements $\left(g_{1}\right.$ and $\left.g_{2}\right)$ and element spacing $(d) \ldots \ldots . \ldots 15$

3.1 Transmission lines considered for DRA array feed . . . . . 17

3.2 Electric field of fundamental DIG and DRA modes . . . . . . 21

3.3 Field profile of $E_{11}^{z}$ mode . . . . . . . . . . . . 22

$3.4 H_{x}$ around DIG's with similar propagation constants . . . . 25

3.5 Design parameters of DIG . . . . . . . . . . . 32

3.6 Dimensions of microstrip fed DRA array for software validation 33

3.7 S-parameters for microstrip fed DRA array . . . . . . . . . 34

3.8 Radiation patterns at $30 \mathrm{GHz}$ for microstrip fed DRA array . 34

viii 
3.9 DIG transitions . . . . . . . . . . . . . . . 37

3.10 New coaxial cable to DIG transition . . . . . . . . . . . . 39

3.11 Insertion loss for setup in Figure $3.10 \ldots . . . . . . . .39$

3.12 DIG without DRA's for characterization . . . . . . . . 43

3.13 S-parameters for DIG with two identical transitions (case $i$ ) . 45

3.14 Gain of the feeding port $($ case $i) \ldots \ldots 46$

4.1 E-field of fundamental modes (ground plane omitted) . . . . 50

4.2 Equivalent circuit for transmission line and resonator . . . . 50

4.3 Directional couplers constructed from DIG's . . . . . . . . 53

4.4 Power density along coupler . . . . . . . . . . . . . 54

4.5 Power along coupler . . . . . . . . . . . . . . 55

4.6 $E_{z}$ component of lowest order even and odd modes . . . . 55

4.7 Summation of even and odd modes . . . . . . . . . . 56

4.8 Typical coupling length curve . . . . . . . . . . 57

4.9 Cross-section of DRA near DIG with identical cross-sections . 58

4.10 Comparison between mode wavelengths and simulated resonant length ....................... 60

4.11 Power coupled from the DIG to a DRA . . . . . . . . . . . 61

4.12 Power budget used to calculate total losses (ground plane omitted) 62

4.13 Power budget used to isolate losses in simulation results . . . . 64

4.14 Manipulation of time domain data to isolate DRA reflection . 67

4.15 Comparison of simulation results to analytical approximation . 72 
4.16 Array fed by non-resonant DIG . . . . . . . . . . 74

4.17 8-element array with uniform amplitude distribution - ARPS . 78

4.18 8-element array with uniform amplitude distribution - Empire 78

4.19 Simulated gain with amplitude distribution in the H-plane . . 78

4.20 8-element array with Chebyshev amplitude distribution - ARPS 81

4.21 8-element array with Chebyshev amplitude distribution - Empire 82

4.22 Simulated gain with Chebyshev distribution in the H-plane . . 82

$4.23 \mathrm{CMT}$ results for DIG at $60 \mathrm{GHz} \ldots \ldots . \ldots . \ldots 84$

4.24 16-element array with Taylor amplitude distribution . . . . . 85

4.25 Microstrip line parameters . . . . . . . . . . . . 88

4.26 Orientations of DRA's with respect to microstrip line . . . . 88

4.27 Relevant H-field components of the microstrip line . . . . . . . 89

4.28 Radiation patterns of $E_{\theta}$ for a single DRA in the $\phi=0^{\circ}$ plane 90

4.29 Design variables for microstrip series feed DRA array . . . . 91

4.30 Radiation patterns for DRA's of different lengths for case $i i \quad$. 93

4.31 Radiation patterns for microstrip line fed DRA array at $30 \mathrm{GHz} 94$

4.32 Co-pol radiation patterns for microstrip line fed DRA array $\quad . \quad 95$

4.33 S-parameters for microstrip line fed DRA array . . . . . . 95

5.1 Effect of DIG loading on radiation pattern . . . . . . . 98

5.2 Lattice with parasitic DRA's . . . . . . . . . . . 99

5.3 Failed options for symmetric loading . . . . . . . 100 
5.4 Radiation patterns for parasitically loaded DRA in the $\phi=0^{\circ}$ plane . . . . . . . . . . . . . . . . . . . . 101

5.5 Lattices of 8-element arrays _. . . . . . . . . . . . 102

5.6 Radiation pattern for staggered DRA array in $\phi=0^{\circ}$ plane . . 103

$5.7 E_{z}$ component of even and odd modes for asymmetric DIG's . 104

5.8 Summation of even and odd modes for asymmetric coupler . . 105

5.9 Metal shorts . . . . . . . . . . . . . . . . . . 106

5.10 Radiation pattern in $\theta=0^{\circ}$ plane with x-pol reduction $\ldots .107$

5.11 X-pol reduction for Taylor array at $60 \mathrm{GHz} \ldots \ldots \ldots . . .108$

5.12 Frequency scanning properties of a linear array . . . . . . 110

5.13 Series-fed array employing phase shifters for each element . . . 111

5.14 Typical output phase for bandpass filters with $0^{\circ}$ input phase 112

5.15 General diagram of N-element array . . . . . . . . . . . . 114

5.16 Gain radiation patterns for microstrip fed DRA arrays _ . . 116

5.17 Beam peak location versus frequency $\ldots \ldots \ldots \ldots$

5.18 Gain in the $\phi=90^{\circ}$ plane in the $\theta=-2^{\circ}$ direction $\ldots \ldots 117$

A.1 Cross section of two DIG's in a waveguide . . . . . . . . 123 


\section{List of Abbreviations}

dBi decibels relative to isotropic

dB decibels

DRA dielectric resonator antenna

DIG dielectric image guide

FDTD finite difference time domain

GHz gigahertz

EHF extremely high frequency $(30-300 \mathrm{GHz})$

EM electromagnetic

E-field electric field

H-field magnetic field

1-D one dimensional

2-D two dimensional

3-D three dimensional

pml perfectly matched layer

CMT coupled mode theory

cpw coplanar waveguide

xii 


\section{Chapter 1}

\section{Introduction}

Designing a low-profile EHF antenna with high gain is a significant engineering challenge. This is primarily due to relatively large transmission line attenuation at high frequencies. Dielectric resonator antenna (DRA) arrays were conceptualized to meet this challenge over two decades ago. Shortfalls in the practical implementation led to arrays without standard amplitude distributions across the elements. DRA theory was not mature. Design rules were non-existent, therefore the antenna remained a concept and not a feasible option for antenna designers bounded by time constraints.

The motivation of this work was to make the DRA array a feasible option for antenna designers. The scope of this thesis was to generate design guidelines for the DRA array. These guidelines were to include the design of the feed network and the DRA's. 
One method of achieving high gain is to use a single high-gain element. Hornfed reflectors or lenses are most commonly used to realize high gain in the EHF range [1]. These systems are not low-profile since the feed is located at a fixed distance away from the reflector or lens.

A second method of realizing high gain is to use several low gain elements in an array. A common problem with arrays operating at EHF is high losses in the feeding mechanism [2]. In addition, the array elements must be efficient radiators at these high frequencies. Systems using microstrip or metallic waveguide components experience increasing conductive and surface roughness losses as the frequency increases, thereby degrading the gain of the system. Furthermore, waveguide and microstrip components decrease in size as the frequency of operation is pushed upward to suppress higher order modes. Small dimensions add complexity, hence increases the cost.

In this work, design guidelines were generated for a dielectric image guide (DIG) fed DRA array. These guidelines governed the parameters that could be varied to achieve specific phase and amplitude distributions across an array. This type of array was compared to a microstrip fed DRA array. Examples of these arrays are shown in Figure 1.1. The guidelines were applied to design arrays with standard amplitude distributions. 


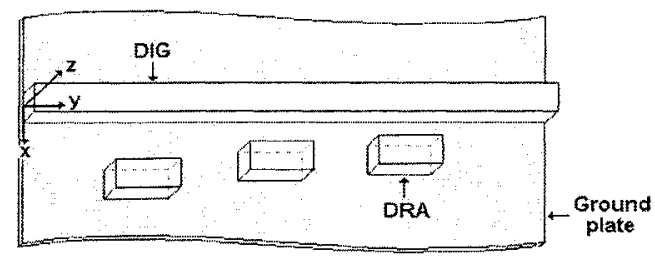

(a) DIG feed

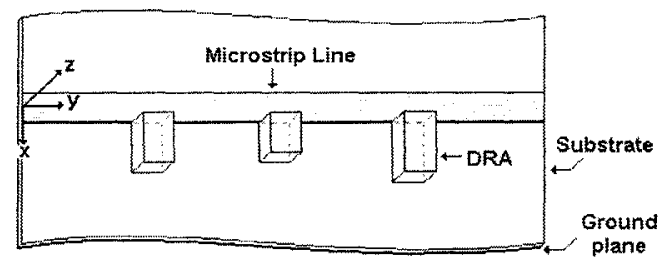

(b) Microstrip feed

Figure 1.1: Layout of two DRA arrays (metallized objects are shaded)

\section{$1.1 \quad$ EHF Band}

The EHF band is designated as $30-300 \mathrm{GHz}$ [3]. This frequency range is vastly unused [1]. Applications in this band can be classified into one of two categories: information communications or remote sensing [4]. Large bandwidth is the attractive feature of the EHF band for information communications. For remote sensing applications, a higher frequency corresponds to a higher angular resolution. The market for applications in the EHF band is significantly smaller than that in the frequency bands that are currently the heaviest populated [1].

Some of the applications that have been allocated within this band are $[1,3]$ :

- vehicle radar

- radio astronomy

- fixed satellite

- non-specific short range device

- wireless access systems
- space operations research

- public/private mobile

- ultra high speed wireless LAN

- motion sensors

- short range radar 


\subsubsection{Types of Antennas}

Applications in the EHF band use a variety of antennas. Some of these antennas are shown in Figure 1.2. As the frequency of operation increases, the performance of some of these antennas degrades. At high frequencies, surface roughness and conductor loss become significant for metallic components, and fabrication tolerances become stricter for waveguides and microstrip. Some applications require a low-profile, low-cost, high-gain antenna to operate in the EHF band. An array of efficient radiators with an efficient feed network and coarse fabrication tolerances would satisfy these requirements. To be considered as a feasible option by antenna designers, design guidelines should exist.

\subsection{DRA Arrays}

An array of DRA's can be used to produce high gain. An array must have a specific amplitude and phase distribution to either maximize the gain or control the sidelobe levels. To achieve the correct phase distribution, the elements were spaced according to the relative phase in the transmission line. The relative phase was a function of the feed line's guided wavelength. To achieve the correct amplitude distribution, each DRA was located at a specific distance away from the DIG to couple out the required amount of power.

A shortfall of several high frequency array designs reported in the literature 


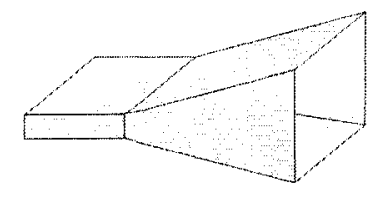

(a) Pyramidal horn

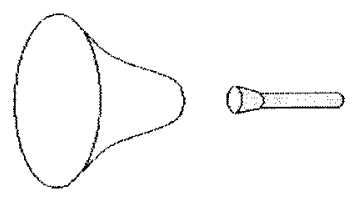

(c) Horn-fed lens

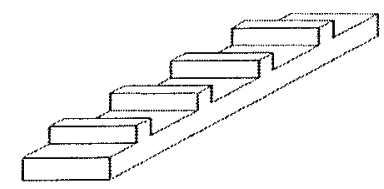

(e) Leaky-wave with dielectric steps

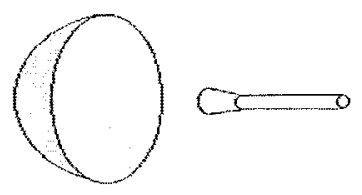

(b) Centre-fed reflector

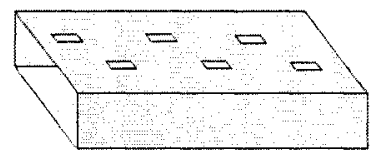

(d) Slotted waveguide

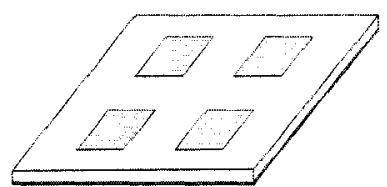

(f) Microstrip patch array

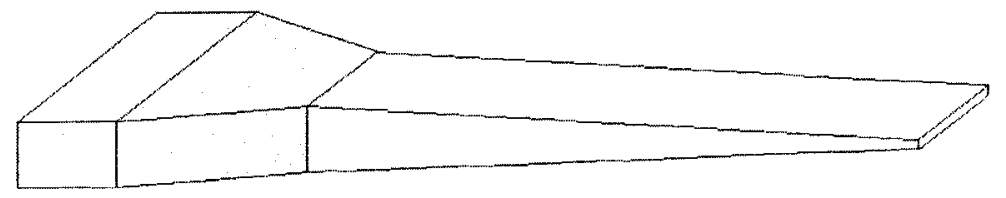

(g) Waveguide-fed dielectric rod with taper

Figure 1.2: Common antennas for EHF applications

was the lack of a standard amplitude distribution across the elements of the array $[5,6,7]$. The term standard distribution is used to describe common amplitude distributions that enhance an array's performance. An example is the uniform distribution for maximum gain [8].

Previous work in DRA arrays has generally positioned all radiating elements equidistant from the feed line $[5,6,7]$. Each element coupled out the same fraction of power from the feed line at the element's location. This situation 
resulted in an amplitude distribution in the form of a decaying exponential function $\left(A_{n}=A_{o} e^{-\alpha x_{n}}\right)$. In this equation, $A_{n}$ corresponds to the amplitude of element $n$, and $x_{n}$ is the position along the array, while the term $\alpha$ is arbitrary. As a comparison the array factors for a 16-element array with a uniform and an arbitrary exponential amplitude distribution are shown in Figure 1.3. The maximum directivity of the uniform distribution was $12.0 \mathrm{dBi}$ and that of the exponential distribution was $9.7 \mathrm{dBi}$. The $2.3 \mathrm{~dB}$ difference is the motivation to be able to excite the DRA elements in Figure 1.1 with specific amplitudes.

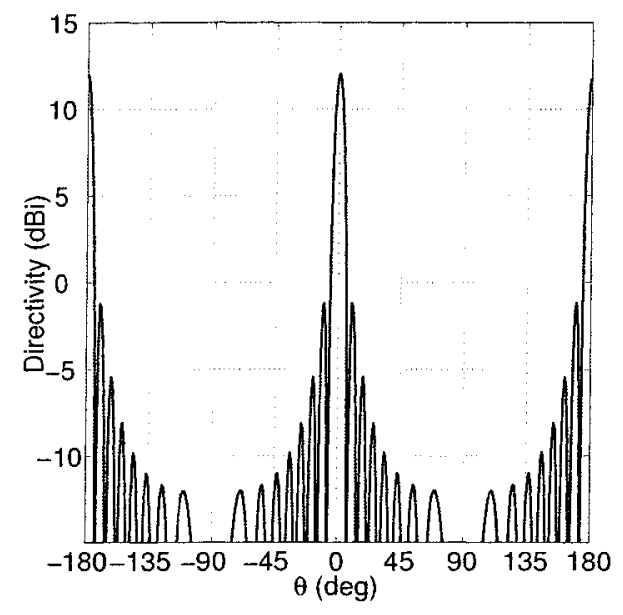

(a) Uniform amplitude distribution

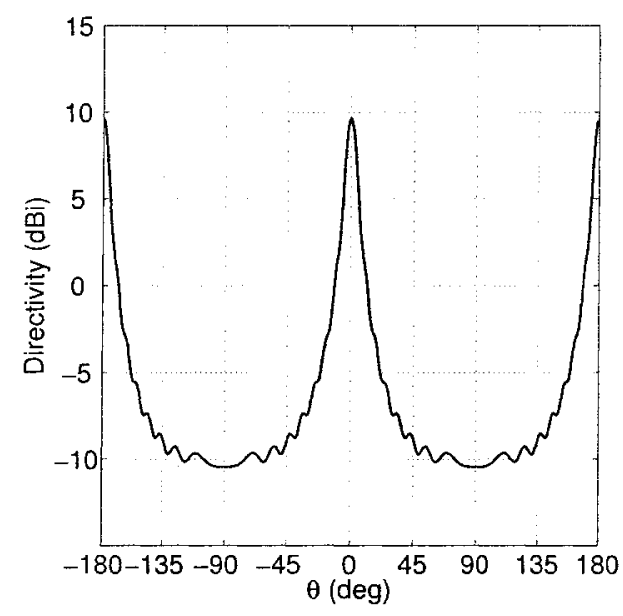

(b) Exponential amplitude distribution

Figure 1.3: Directivity of array factors in array plane

\section{Thesis Overview}

This thesis consists of four chapters bookended by the introduction and conclusion. The following two chapters provide a background of the relevant topics, and insight into the early design decisions. The latter two chapters contain the contributions and results. The design guidelines are summarized in the conclusion. 


\section{Chapter 2}

\section{The Dielectric Resonator}

\section{Antenna}

Dielectric resonator antennas have been shown to radiate efficiently in the EHF band [9]. These antennas exhibit less conductor loss than printed antennas and have a lower profile than lenses or reflectors fed by horns.

\subsection{DRA Survey}

DRA's are categorized based on their geometry. Figure 2.1 shows several types of DRA's. Each type of DRA will couple different amounts of energy out of a specific transmission line. The radiation modes for all DRA types are similar therefore the radiation patterns are similar. 


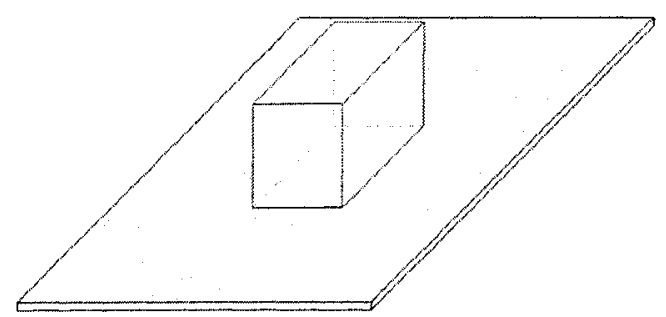

(a) Rectangular

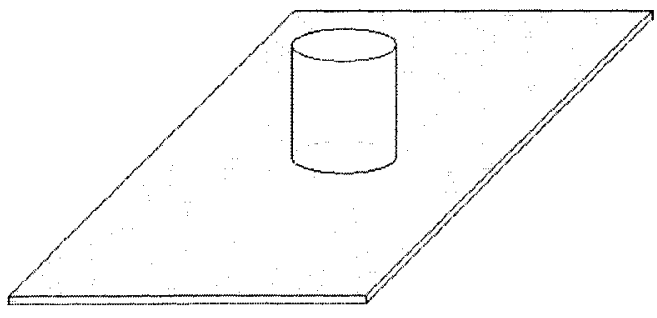

(c) Cylindrical

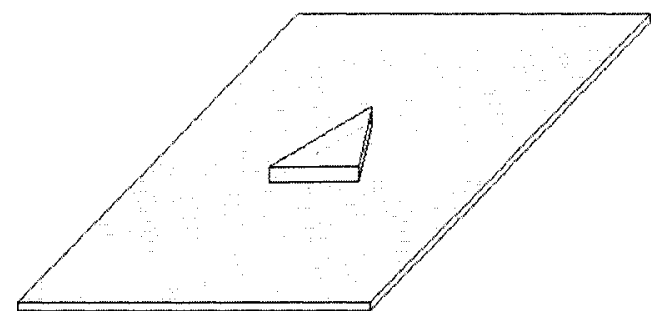

(b) Triangular

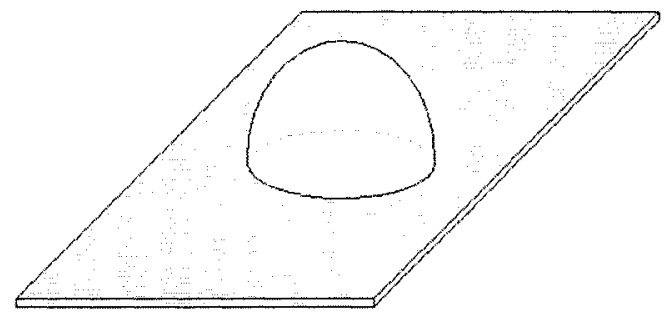

(d) Hemispherical

Figure 2.1: Types of DRA's considered

\section{Rectangular}

Rectangular DRA's are a practical option for the antenna designer since they offer both ease of fabrication, and there are two degrees of freedom in the design [10]. The drawback of this type of DRA is with regards to the analysis. Due to the sharp corners, it is difficult to perfectly match all of the boundary conditions therefore it does not have a closed form solution for the Green's function. The dielectric waveguide model is an approximation that is generally used to determine the resonant frequency of the rectangular DRA [10]. 


\section{Cylindrical}

A common geometry for the DRA is a cylinder [11]. This type of DRA is easy to manufacture from a dielectric rod. Due to it's rotational symmetry, it lends itself to a body of revolution analysis (BOR) [12]. After the material's permittivity is chosen, the resonant frequency depends on the radius and height. The major disadvantage of this type of DRA is that the ratio of radius to height cannot be arbitrary since the pattern shape is dependent on this value. This leaves one degree of freedom in the design, but with restrictions. Furthermore, mode degeneracy in cylindrical DRA's is present and can result in high cross-polarization levels [13].

\section{Triangular}

The size of the triangular DRA is smaller than the rectangular and cylindrical DRA for a specific dielectric constant [14]. This characteristic is not advantageous for this work since the frequencies are very high, therefore the size of the DRA's are already small, and if they are made smaller then fabrication will become more complex. The work done with triangular DRA's has been for low-profile equilateral triangles $[14,15]$, where the resonance frequency becomes a function of only the height and permittivity. This results in no degree of freedom in the design after either the permittivity or height have been selected. 


\section{Hemispherical}

Closed formed expressions exist for the Green's function of the hemispherical DRA [16]. This type of DRA is difficult to fabricate, therefore is not deemed practical for this work. Furthermore, there is no degree of freedom in the design, so it is not an attractive option compared to cylindrical and rectangular DRA's. It is more useful to study analytically since many of the trends are similar for other types of DRA's.

\subsubsection{Survey Results}

Rectangular DRA's were chosen for this work. For the DRA array there is some dependence on the characteristics of the feeding transmission line, therefore it is desirable to have the extra degrees of freedom for the DRA. Ease of fabrication is another driving factor to be cost effective. The closed form solutions that exist for the hemispherical DRA and the BOR analysis for the cylindrical DRA do not account for the loading that the transmission line applies to the DRA's, therefore the solutions may be inaccurate. The transmission lines considered for this work supported hybrid modes, therefore higher order modes in the DRA were excited. The dimensions of the rectangular DRA's can be adjusted to shift the resonant frequency of the undesired radiating modes away from the operating frequency. 


\subsection{Theory of Rectangular DRA's}

The dipole modes of the rectangular dielectric resonator are mathematically complex, so no closed-form solution exists. One technique that had been used to model this problem was by considering the resonator to be a truncated DIG [10]. Marcatili's method [17] was used to analyze the equivalent DIG. This method of analysis is referred to as the dielectric waveguide model (DWGM). The dimensions and axis are labelled on the DRA shown in Figure 2.2.

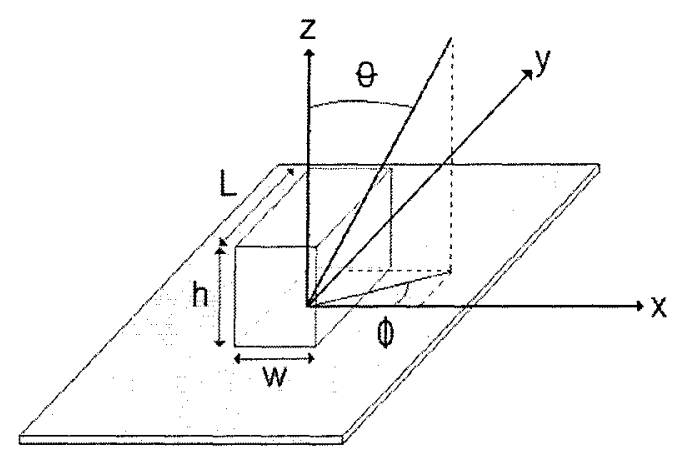

Figure 2.2: Rectangular DRA with dimensions

\subsubsection{Resonant Frequency}

The resonant frequency of the $T E_{\delta 11}^{x}$ mode is related to the dimensions and permittivity of the DRA by the relationships in (2.1)-(2.3) [10]. The $T E_{1 \delta 1}^{y}$ mode can be analyzed using the same equations, but $x$ must be swapped with $y$, and $w$ with $L$. An assumption that is part of the DWGM is that the fields are well confined in the $y$ and $z$ dimension. This assumption is applied by placing magnetic walls at the boundaries of the DRA that correspond to these 
dimensions. The transversal wavenumbers $\left(k_{y}\right.$ and $\left.k_{z}\right)$ are:

$$
k_{y}=\frac{\pi}{L} \quad k_{z}=\frac{\pi}{2 h}
$$

The general dispersion relationship is:

$$
k_{x}^{2}+k_{y}^{2}+k_{z}^{2}=\epsilon_{r} k_{o}^{2}
$$

From matching the boundary conditions and using Marcatili's approximation [17], the transcendental equation governing the wavenumber along the axis of propagation $\left(k_{x}\right)$ for the equivalent dielectric waveguide is:

$$
k_{x} \tan \frac{k_{x} w}{2}=\sqrt{\left(\epsilon_{r}-1\right) k_{o}^{2}-k_{x}^{2}}
$$

\section{Bandwidth}

The analytical method of calculating the bandwidth requires the quality factor $(Q)$ for the DRA. If the $Q$ of the DRA is high, then it will act like a resonator instead of a radiator and will reflect most of the energy back towards the generator. The impedance bandwidth of the DRA is not particularly useful in this work since the fields of the feeding mechanism change with frequency. It is useful to see the effect that the design parameters have on how efficiently the DRA radiates. An analytical approximation for $Q$ was derived in [10]. For maximum radiation efficiency, a low $Q$ is desirable. The plots of $Q$ are reported in [18] for different geometries and permittivities. These plots show 
the variation of the $Q$ with respect to $L / h$ and $w / h$, but in general a low permittivity results in a low $Q$.

\subsubsection{Radiation}

In this work the rectangular DRA was operated by exciting the $T E_{\delta 11}^{x}$ and $T E_{1 \delta 1}^{y}$ modes. These modes are magnetic dipole modes, and therefore were assumed to radiate like short magnetic dipoles. The radiation pattern in Figure 2.3 shows the ideal pattern for an $x$-oriented magnetic dipole in the principle planes. The $\theta$ and $\phi$ angles are defined in Figure 2.2, and an infinitely large ground plane approximation applies.

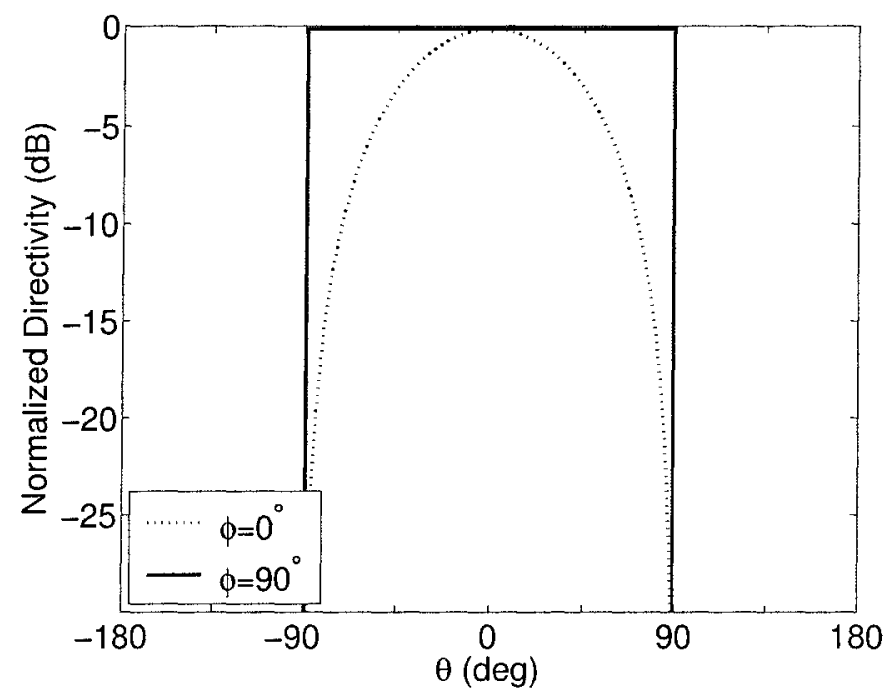

Figure 2.3: Ideal normalized radiation pattern for $T E_{\delta 11}^{x}$ mode 


\subsubsection{Mutual Coupling}

Mutual coupling in an array is the coupling of power between elements. Generally this type of coupling is undesirable. To reduce this effect the element spacing is increased. Mutual coupling effects are important to investigate in an array environment to determine the minimum element spacing. Studies of mutual coupling with DRA's have been reported $[19,20,21]$. In these papers, two elements were connected to separate ports. The mutual coupling was quantified by $s_{21}$, where port 1 was excited. The results showed that when the spacing between two elements was greater than $\frac{\lambda_{0}}{2}$ the $s_{21}$ was always better than $-12 \mathrm{~dB}$ regardless of whether the DRA's were oriented in the H-plane or E-plane.

Mutual coupling was not just a function of the element spacing, but also of the DRA parameters. Preliminary simulations showed that two elements would strongly couple when one of the DRA dimensions was large enough such that the actual gap between the elements became relatively small. Two arrays are shown in Figure 2.4 with equal element spacing but different DRA dimensions, and therefore exhibit different levels of mutual coupling. In this Figure the gap between the DRA's is smaller for array 1 than array $2\left(g_{1}<g_{2}\right)$, as a result it was found that the mutual coupling was stronger in array 1 than array 2 .

An unpleasant effect of mutual coupling in DRA arrays is the tendency to shiftup the resonant frequency. Furthermore, the coupling between elements degrades an array's amplitude distribution since each element is receiving power 


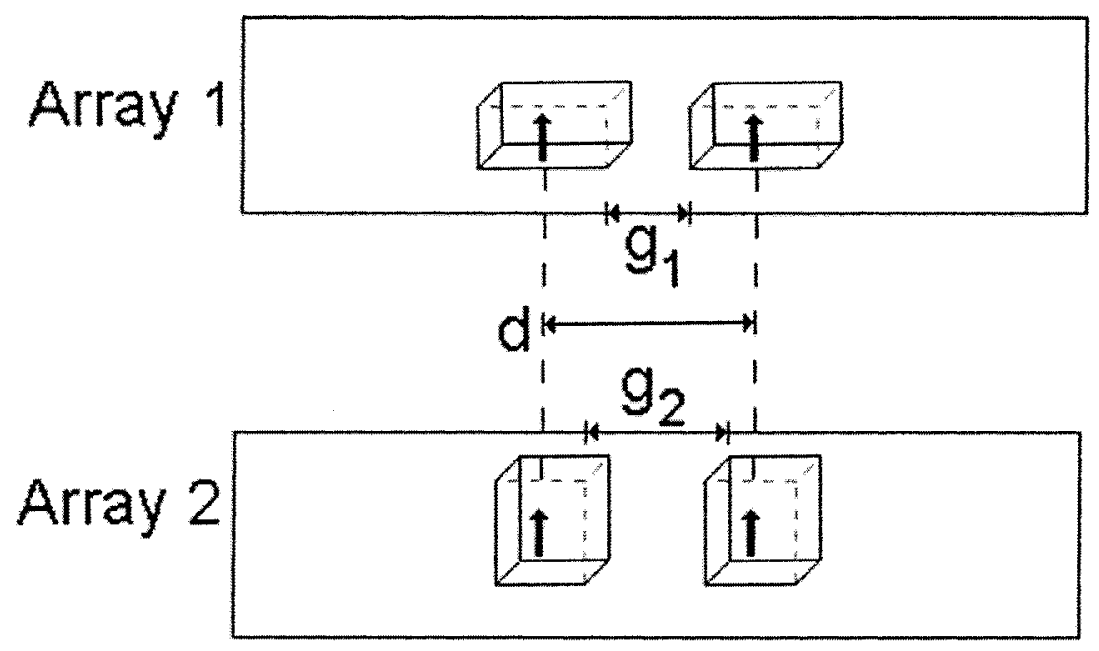

Figure 2.4: Two 2-element arrays showing physical gap between elements $\left(g_{1}\right.$ and $\left.g_{2}\right)$ and element spacing $(d)$

from sources other than the feeding line. Simulation results were relied upon to determine the minimum spacing between elements, since an analytical approach would not have been as efficient - timewise.

\section{Chapter Summary}

This chapter investigated different types of DRA's. The rectangular DRA was chosen for this thesis work. Methods to determine the resonant frequency, radiation pattern and bandwidth for the DRA's were discussed. The undesirable effects of mutual coupling between DRA's was investigated, and what is required to reduce this type of coupling. 


\section{Chapter 3}

\section{The Feed Network}

The task of the feed network was to adequately excite the DRA elements with minimal degradation to the gain of the array. For this task the feeding transmission line was required to support a loosely bound mode for good power coupling into the DRA's, and to exhibit low loss characteristics. A linear series feed was employed due to its superior loss characteristics for large arrays when compared to a corporate feed.

\subsection{Transmission Line Survey}

Several types of transmission lines were investigated for the series-fed DRA array. The characteristics that were considered to be most important for this work were low-loss, ease of fabrication and good coupling characteristics 
with a DRA located in proximity to the transmission line. To ensure the latter characteristic, only open waveguiding structures were considered. The transmission lines considered are shown in Figure 3.1.

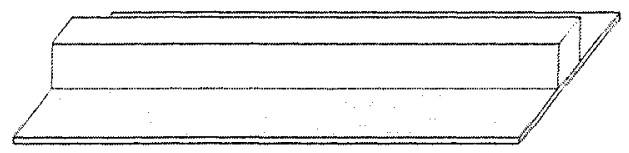

(a) DIG

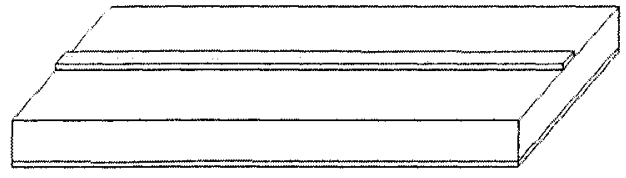

(c) Microstrip line

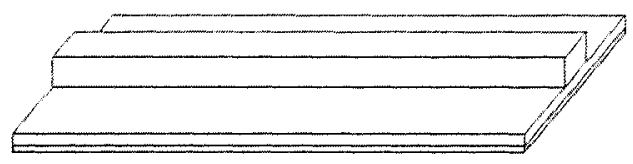

(b) Insular DIG

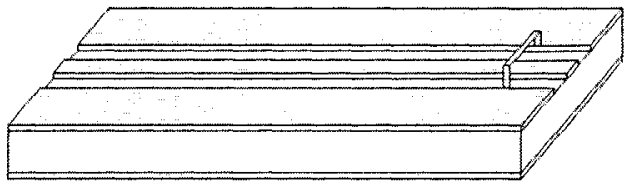

(d) CPW

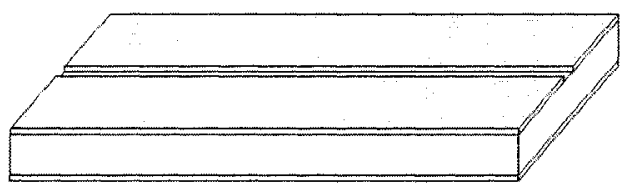

(e) Slot line

Figure 3.1: Transmission lines considered for DRA array feed

\section{Dielectric image guide}

The dielectric image guide (DIG) is a rectangular dielectric waveguide mounted on a ground plane. The DRA can be considered as a truncated DIG, and the fields of the fundamental modes of the DIG and DRA are similar. A DIG can be designed so that most of the energy propagates in the region outside of the guide, thereby reducing dielectric loss [22]. DIG's designed so that most of the energy propagates outside of the guide experience significant radiation losses at discontinuities. The two advantages that the ground plane brings to 
the DRA array are firstly, it confines the problem above the ground plane, ideally doubling the directivity. Secondly, the nearly degenerate mode that exists in a dielectric waveguide is shorted out [22], so the DIG can be designed to support only one mode. The ground plane introduces conductor loss for the fundamental mode since it has a magnetic field component tangential to the conducting surface. Fabrication problems inherent to DIG's are the presence of an air gap between the ground and dielectric material, and the requirement to use lossy adhesives to bond these two materials together. The fabricated DIG must be connected to the test equipment, therefore an input transition is required to couple the energy from a coaxial cable to the DIG.

\section{Insular dielectric image guide}

The difference between the insular DIG and the DIG is the addition of a lower permittivity substrate inserted between the dielectric guide and the ground plane. The energy is guided in the line, but the substrate is intended to reduce conductor loss by acting as a buffer layer between the ground and the maximum tangential magnetic field components of the fundamental mode [23]. Generally conductor loss is lower for an insular DIG when the energy is tightly confined just above the ground plane. For a loosely bound mode, the mode profile of the insular DIG looks very similar to that of a DIG, and therefore has similar conductor loss [24]. In this thesis work the mode is loosely bound. In comparison to the DIG, this type of transmission line would increase the complexity and load the DRA, thereby changing the resonant frequency. 


\section{Microstrip}

Microstrip line is a mature technology at microwave frequencies [25]. Fabrication techniques such as photolithograpy and etching have been applied to make cost-effective microstrip line circuits [25]. As the frequency increases into the EHF band the substrate permittivity, substrate thickness and strip width must decrease to suppress higher order modes. These dimensions can become prohibitively small. Attenuation increases with frequency and is primarily from conductor loss and the excitation of surface-waves in the substrate. A low-loss transition for coaxial cable to microstrip is relatively easy to design.

\section{Coplanar waveguide}

Coplanar waveguides (CPW) offer the advantage of having all of the conductors on one side of the substrate, thereby decreasing the dependence of the performance of the line from the substrate parameters. A common configuration is to use a conductor backed substrate, but at high frequencies the substrate will be very thin and an additional ground plane would effect the operation of the CPW. Without a conductor backed substrate there will be

unwanted back radiation. The major drawback of the CPW is the presence of two fundamental modes that exist with no cut-off frequency. To suppress one of these modes an air-bridge is required to keep the potential on both ground planes equal in polarity [26]. An air-bridge is shown on the CPW in Figure 3.1(d). These bridges present discontinuities to the line, therefore they cause 
radiation and the excitation of surface-waves. Air-bridges add complexity to the manufacturing process.

\section{Slotline}

The slotline is the complement of the microstrip line. A slotline supports a quasi- $T E_{10}$ type dominant mode, which is similar to the $T E_{10}$ mode of the rectangular waveguide. The dominant field components are similar to the DRA, therefore is useful for coupling into the DRA. The slotline is more dispersive and lossier than microstrip line [26]. The same issues arise with regard to a conductor backed substrate or lack thereof as with the CPW.

\subsubsection{Survey Results}

The DIG was chosen to use as the feed network for the DRA array due to its low-loss properties and the similarities in the fields between the fundamental modes of the DIG and DRA. These similarities help facilitate coupling, but only apply when the DRA is oriented such that the equivalent magnetic dipole of the mode is perpendicular to the DIG. The fields are shown inside the DIG and DRA in Figure 3.2. The microstrip line was chosen to feed the DRA array to compare the performance to the DIG fed array.

The equipment used to test the antenna had coaxial cables as the transmission medium. For the microstrip line, mounting a k-connector to the substrate 


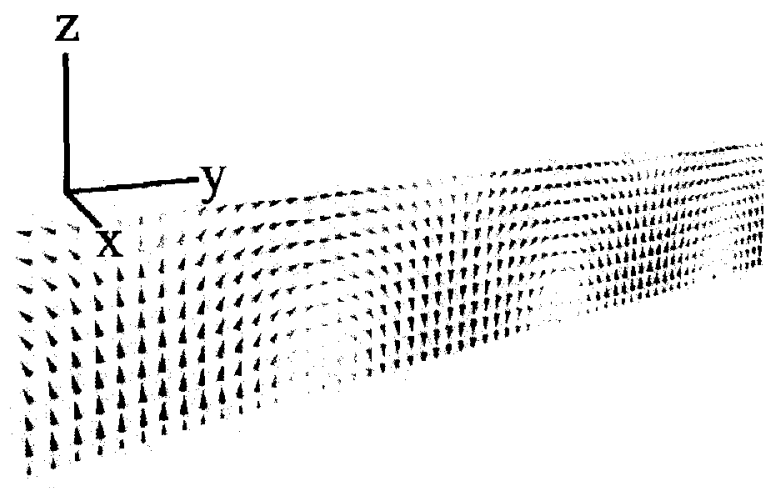

(a) $E_{11}^{z}$ of DIG

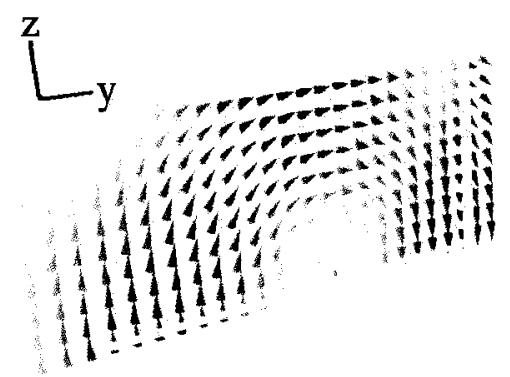

(b) $T E_{\delta 11}^{x}$ of DRA

Figure 3.2: Electric field of fundamental DIG and DRA modes

provided an adequate transition to couple the energy into the feed network. The DIG proved to be more of a challenge since coaxial-to-DIG transitions were not as common as k-connectors.

\subsection{Characterization of the DIG}

The most important properties of a transmission line for use as a DRA array feed are the loss and coupling properties. The guided wavelength is also of importance since the element spacing is based on this value to get a specific phase distribution. The guided wavelength of the DIG should not force the spacing between radiating elements to be too close since mutual coupling would be significant, nor should it force the spacing to be too large such that grating lobes become visible. Also, the DIG must be designed to only support a single mode since higher order modes will couple less power into the DRA due to their mismatch in field configurations. If not The field profile of the fundamental 
mode is shown in Figure 3.3.
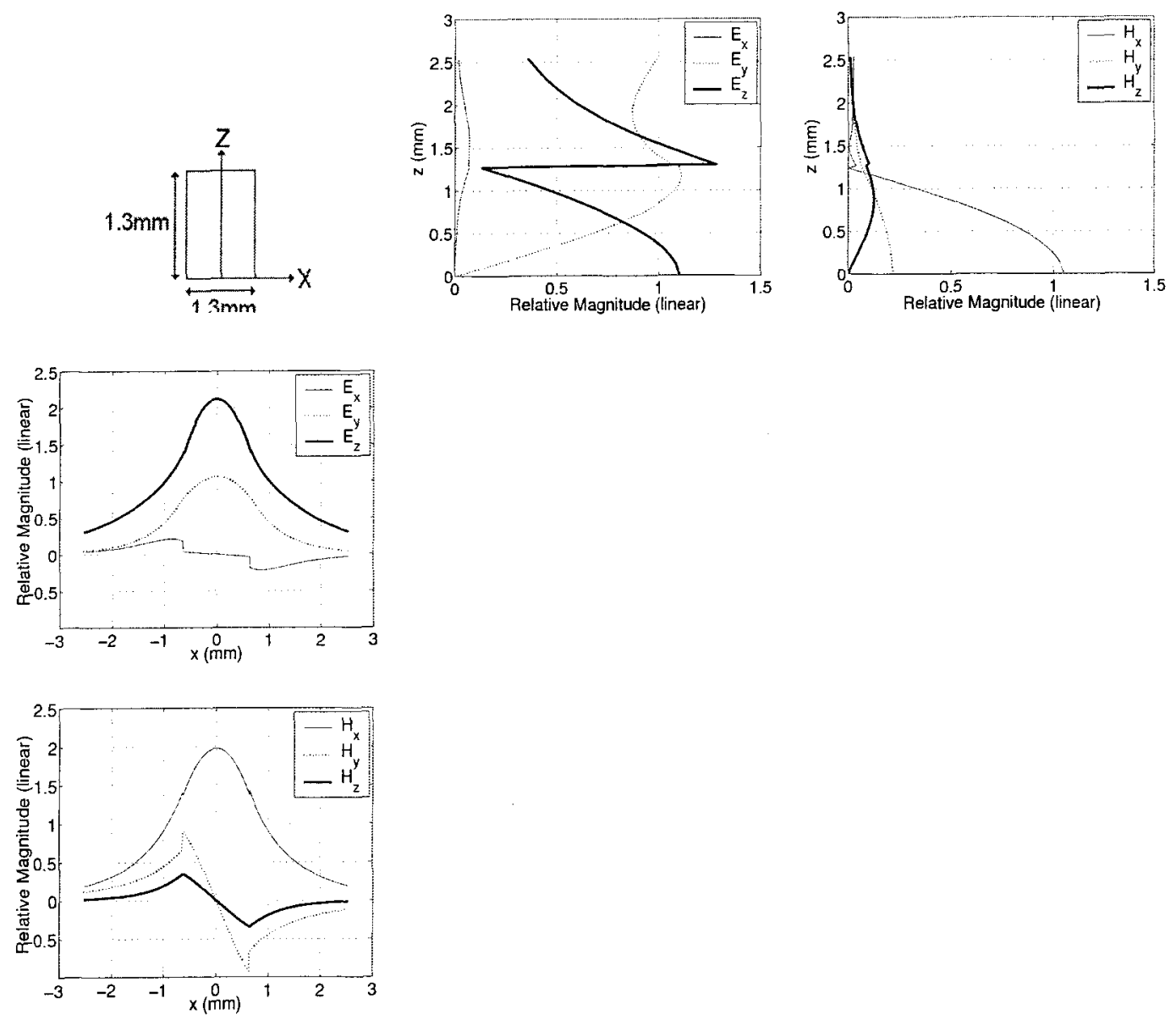

Figure 3.3: Field profile of $E_{11}^{z}$ mode 


\subsubsection{Characteristic Parameter}

A DIG with lossless materials can be characterized by a single parameter. The parameter can be expressed as either the guided wavelength $\left(\lambda_{g}\right)$, phase velocity $\left(v_{p}\right)$ or axial propagation constant $\left(\beta_{y}\right)$. The relationships between these three parameters are shown in (3.1). The terms $\lambda_{o}, c$ and $k_{o}$ represent the free space wavelength, speed of light and free space wave-number, respectively.

$$
\frac{\lambda_{g}}{\lambda_{o}}=\frac{v_{p}}{c}=\frac{k_{o}}{\beta_{y}}
$$

The characteristic parameter has two important ramifications. Firstly, the guided wavelength of the DIG plays a critical role with regard to the phase distribution along the array. For broadside radiation the spacing must be some integer multiple of $\lambda_{g}$ to ensure the elements are in phase. Gain degradation will occur due to mutual coupling if the element spacing is too small, or due to the onset of grating lobes if the spacing is too large. From array theory the relationship between the phase progression $(\beta)$, element spacing $(d)$ and main beam direction with respect to broadside $\left(\theta_{o}\right)$ is given by $(3.2)$.

$$
-\beta=k_{o} d \sin \theta_{o}
$$

The phase progression is a function of the element spacing and the guided wavelength of the DIG.

$$
\beta=\left(d-\lambda_{g}\right) \frac{2 \pi}{\lambda_{g}}
$$


The second important ramification of the characteristic parameter is that it determines how loosely bound the energy is to the DIG. A loosely bound mode refers to a propagating mode where most of the energy is travelling outside of the dielectric material, and a tightly bound mode refers to that where most of the energy is confined within the dielectric material. For good power coupling from the DIG into the DRA's it is beneficial to have a loosely bound mode on the DIG.

$$
\begin{aligned}
& \text { Tightly bound: } \frac{\lambda_{g}}{\lambda_{o}}=\frac{v_{p}}{c}=\frac{k_{o}}{\beta_{y}}<<1 \\
& \text { Loosely bound: } \frac{\lambda_{g}}{\lambda_{o}}=\frac{v_{p}}{c}=\frac{k_{o}}{\beta_{y}} \approx 1
\end{aligned}
$$

Designing a DIG with a loosely bound mode does not guarantee the conditions required for good coupling. As an example the cross-section of two DIG's are shown in Figure 3.4. Also shown in this Figure are the magnitudes of the $z$-directed electric field for the fundamental mode. Both DIG's have similar values of $k_{o} / \beta_{y}$ but most of the energy external to the first guide is propagating above the DIG, and for the second guide most is propagating alongside the DIG. The latter case will have better coupling since the field strength is stronger at the location of the DRA's.

The major drawback of a loosely bound surface-wave is the accompanying decrease in feed efficiency. A surface-wave is classified as loosely bound if it has a phase velocity $\left(v_{p}\right)$ that is near that in free space $(c)$. Due to this characteristic the energy will easily couple into radiating modes $\left(v_{p}=c\right)$ at discontinuities. 


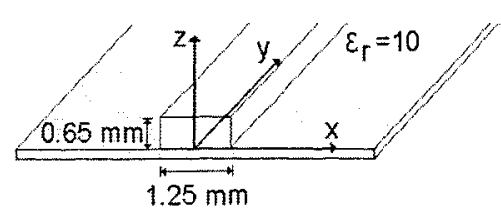

(a) Small aspect ratio

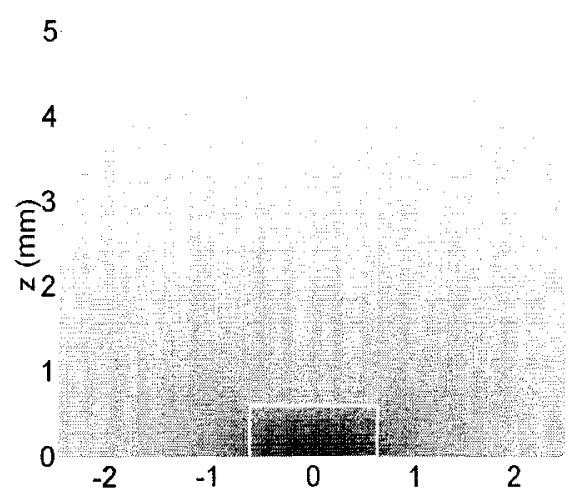

(c) Fields for small aspect ratio

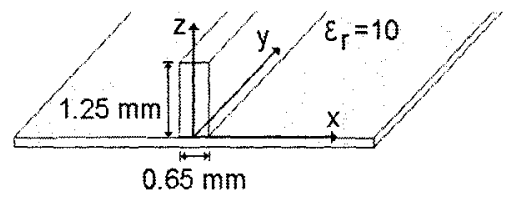

(b) Large aspect ratio

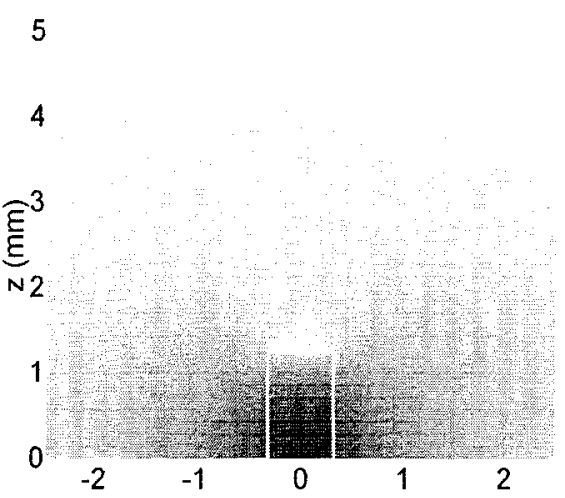

(d) Fields for large aspect ratio

Figure 3.4: $H_{x}$ around DIG's with similar propagation constants

The transition from a coaxial cable to the DIG contains discontinuities, therefore radiation losses will be significant for a DIG that supports a loosely bound mode.

The characteristic parameter that is generally calculated for DIG's is $\beta_{y}[17$, $27,28,29]$. The first method used to calculate $\beta_{y}$ analytically for rectangular DIG's was developed by Marcatili in 1969 [17]. In this method it was assumed that the mode was tightly confined to the DIG. In 1970, Knox and Toulious introduced the effective dielectric constant (EDC) method [27]. The EDC technique is relatively simple to apply to DIG's since the most complicated step is calculating the propagation constant of dielectric slabs. Two major assumptions are made in the EDC method of analysis. Firstly, it is assumed that 
the width of the DIG is greater than the height [28]. Secondly, it is assumed that the surface-wave mode is tightly bound [28]. Both of these conditions contradict the conditions required for good coupling, therefore do not apply to the DIG's in this work. A rigorous mode-matching method was employed to determine $\beta_{y}$ based on work by Solbach and Wolff in 1978 [29]. The final results from [29] were not complete enough to directly code, therefore the derivation was repeated for a more general case, and is attached as appendix A. This derivation was completed for two parallel DIG's. The dimensions and permittivity of the two guides can be different. To calculate $\beta_{y}$ for an isolated DIG, the separation distance between the DIG's is set to a large value. The derivation was coded into MATLAB. The output of the code was the propagation constants of all propagating modes as well as the field distributions in and around the DIG's. The code was validated for the fundamental mode using Empire and the results from [29].

\subsubsection{Material Losses}

DIG loss is due to non-ideal material properties. These properties include: finite conductivity of ground plane, non-zero dissipation factor of dielectric materials and surface roughness on both the dielectric material and the ground plane. These non-ideal material properties cause measurable loss that varies with the design parameters, such as the DIG dimensions, permittivity and frequency. The goal of this section was to investigate DIG loss. Surface roughness loss was not investigated. 


\section{Conductor loss}

The $E_{11}^{z}$ mode of a DIG has a relatively large $H_{x}$ component at the interface of the ground plane and dielectric. This component is tangential to the ground plane, therefore induces an electric current. The ground plane has a finite conductivity, therefore the induced current will dissipate energy by means of heat. The attenuation constant for conductor loss in a transmission line $\left(\alpha_{c}\right)$ is given by (3.5) [27]. In this equation $W_{l}$ represents the power lost per unit length, and $W_{t}$ represents the power transmitted by the transmission line per unit length.

$$
\alpha_{c}=\frac{W_{l}}{2 W_{t}}
$$

The power lost per unit length depends on the magnitude of the induced surface current in the metal, and the conductive property of the metal. A perturbation technique was used that assumed that the finite conductivity of the ground plane did not significantly change the fields of the $E_{11}^{z}$ mode. The induced surface current was determined from an electromagnetic boundary condition. This boundary condition is shown in (3.6), where $\vec{J}_{s}$ is the surface

current, $\vec{H}_{t a n}$ is the magnetic fields tangential to the surface of the conductor, and $\hat{n}$ is a unit vector perpendicular to the surface of the conductor. Both $\vec{J}_{s}$ and $\vec{H}_{t a n}$ and in units of $\mathrm{A} / \mathrm{m}$.

$$
\begin{aligned}
\vec{J}_{s} & =\hat{n} \times \vec{H} \\
\therefore & \left|J_{s}\right|=\left|H_{\text {tan }}\right|
\end{aligned}
$$


The power dissipated in a resistive medium is governed by Ohms law. The dissipation per unit length is given by (3.7), where $R_{s}$ is the surface resistance of the metal.

$$
W_{l}(x)=\frac{R_{s}\left|J_{s}(x)\right|^{2}}{2}
$$

To use (3.6) in (3.7) the magnitude of the tangential magnetic field was required. The tangential $\mathrm{H}$-field varies at the interface along the $x$-direction, and is the vector summation of the $H_{x}$ and $H_{y}$ components.

$$
\left|H_{t a n}(x)\right|=\sqrt{\left|H_{x}(x)\right|^{2}+\left|H_{y}(x)\right|^{2}}
$$

To calculate the sum of the total tangential field the expression in (3.8) was integrated over the width of the ground plane. Combining (3.6) - (3.8) gives an expression for $W_{l}$ as a function of the tangential magnetic fields and the surface resistivity. This expression is shown in (3.9). The relationship between surface resistivity and conductivity $(\sigma)$ is given by $(3.10)$.

$$
\begin{gathered}
W_{l}=\frac{R_{s}}{2} \int_{\text {ground plane }}\left(\left|H_{x}(x)\right|^{2}+\left|H_{y}(x)\right|^{2}\right) d x \\
R_{s}=\sqrt{\frac{2 \pi f \mu_{0}}{2 \sigma}}
\end{gathered}
$$

The power guided by the DIG was calculated by integrating the Poynting vector, $\vec{S}$ over the entire cross-section of the open guide. This cross-section 
corresponded to the $x z$-plane.

$$
\begin{aligned}
& \vec{S}=\frac{1}{2} \operatorname{Re}\left\{\vec{E} \times \vec{H}^{*}\right\} \\
& \therefore W_{t}=\frac{1}{2} \int_{x z-\text { plane }} \hat{y} \cdot \operatorname{Re}\left\{\vec{E} \times \vec{H}^{*}\right\} d S \\
& =\frac{1}{2} \int_{x z-\text { plane }}\left|\operatorname{Re}\left\{E_{x}(x, z) H_{z}^{*}(x, z)-E_{z}(x, z) H_{x}^{*}(x, z)\right\}\right| d S
\end{aligned}
$$

Substituting the results from (3.9) and (3.11) into (3.5) yields the expression for conductor loss in terms of the electric and magnetic fields, and the surface resistivity. The units for $\alpha_{c}$ are nepers $/ \mathrm{m}$.

$$
\alpha_{c}=\frac{\frac{R_{s}}{2} \int_{\text {ground plane }}\left(\left|H_{x}(x)\right|^{2}+\left|H_{y}(x)\right|^{2}\right) d x}{2 \times \frac{1}{2} \int_{x z-\text { plane }}\left|\operatorname{Re}\left\{E_{x}(x, z) H_{z}^{*}(x, z)-E_{z}(x, z) H_{x}^{*}(x, z)\right\} d S\right|}
$$

The field components required for (3.12) are available from the mode-matching code based on the derivation in appendix $\mathrm{A}$.

\section{Dielectric loss}

The attenuation due to dielectric loss for a DIG $\left(\alpha_{d}\right)$ is related to the power confinement in the guide as well as the permittivity and the loss tangent $(\tan \delta)$

of the material. The dielectric loss in an infinite isotropic medium $\left(\alpha_{d 0}\right)$ is calculated using (3.13). For a DIG this loss would be a good approximation if 
the propagating mode was tightly bound.

$$
\alpha_{d 0}=\frac{\pi \sqrt{\epsilon_{r}} \tan \delta}{\lambda_{0}}
$$

For the $E_{11}^{z}$ mode of the DIG there exists evanescent regions outside of guide. These regions contain power that is propagating along the axial direction. This power is propagating in air as opposed to the dielectric material, hence experiences free space permittivity and negligible dissipation. In this work the DIG's were designed to support loosely bound modes, therefore a significant amount of the power propagates outside of the dielectric material and (3.13) is not a valid approximation.

A common method for calculating dielectric loss is by introducing a complex dielectric constant $\left(\bar{\epsilon}_{r}\right)$ into the derivation of the modal propagation constants $[27,30]$. The result is a complex propagation constant $\left(k_{y}\right)$.

$$
\begin{aligned}
& \overline{\epsilon_{r}}=\epsilon_{r}(1-j \tan \delta) \\
& k_{y}=\beta_{y}-j \alpha_{d}
\end{aligned}
$$

The first step to calculate $k_{y}$ was applying the mode-matching analysis for a lossless DIG to determine $\beta_{y}$. The complex permittivity was then introduced, and a complex component was added to the propagation constant $\left(\alpha_{d}\right)$. The real part of $k_{y}$ was kept constant, and the complex part was varied to find the zero of the characteristic equation. 


\subsubsection{Results for Specific DIG's}

All of the previous work published for this type of antenna has encountered problems with coupling significant amounts of power from the DIG into the DRA's. The two conditions for good coupling between the DIG and DRA were discussed in the previous section:

- The DIG must support a loosely bound mode.

- The aspect ratio of the DIG must be relatively large.

A DIG with a small cross-section and low permittivity supports a loosely bound surface-wave. To ensure that a relatively simple manufacturing process could be used, the dimensions of the DIG could not be too small. Furthermore, since dielectric material comes in standard thicknesses, it is preferable to have one dimension that corresponds to one of these standard values. THe material characteristics were based on Rogers RT6010 $\left(\epsilon_{r}=10.2 \pm 0.25, \tan \delta=0.0023\right)$ and RT6006 $\left(\epsilon_{r}=6.15 \pm 0.15, \tan \delta=0.0027\right)$. This material comes in thicknesses of $100,75,50,25$ and 10 mil. The latter two values are so small that the manufacturing process would have been prohibitively complex for a long DIG.

Table 3.1 summarizes four DIG geometries and their characteristics. Simulations with an EM solver were used to validate the theory. The EM solver is discussed in the following section. The design of the surface-wave launcher used in the simulations is discussed in section 3.4. 


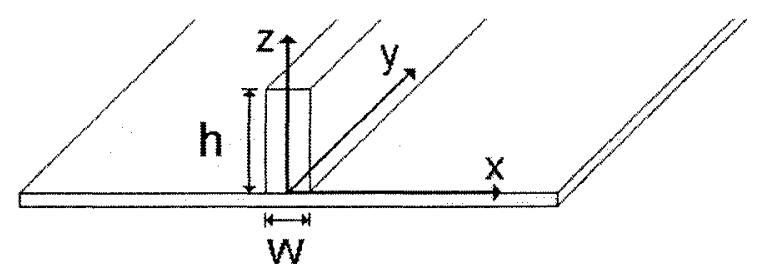

Figure 3.5: Design parameters of DIG

Table 3.1: Design parameters for the DIG's

\begin{tabular}{|c|c|c|c|c|c|c|}
\hline Case & $\mathrm{h}(\mathrm{mm})$ & $\mathrm{w}(\mathrm{mm})$ & $\epsilon_{r}$ & $\lambda_{g}(\mathrm{~mm})$ & $\alpha_{d}(\mathrm{~dB} / \mathrm{cm})$ & $\alpha_{c}(\mathrm{~dB} / \mathrm{cm})$ \\
\hline \hline$i$ & 1.27 & 1.27 & 10 & 6.52 & 0.26 & 0.027 \\
\hline$i i$ & 1.27 & 1.905 & 10 & 5.19 & 0.33 & 0.029 \\
\hline$i i i$ & 1.905 & 1.27 & 6 & 7.63 & 0.17 & 0.011 \\
\hline$i v$ & 2.54 & 1.0 & 10 & 5.05 & 0.63 & 0.001 \\
\hline
\end{tabular}

\subsection{Empire}

The purpose of this section was to validate the simulation tool that was used throughout this thesis work. The simulator used to predict the EM behaviour was Empire [31]. This 3-D field simulator is based on the finite difference time domain (FDTD) method. The FDTD method replaces the derivatives in Ampere's and Faraday's laws with finite differences [32]. The space and time domain are discretized to stagger the electric and magnetic fields in these domains. The time domain is discretized into time steps. The fields for a specific time step are calculated based on the solution of the fields at the previous time step. Empire allows the user to choose the total number of time steps. The model is excited with a Gaussian pulse with a user-defined bandwidth. The 3-D geometry is meshed into rectangular blocks called Yee 
cells. For non-rectangular objects many Yee cells are needed to represent the actual geometry, which results in dense meshing.

A 6-element DRA array that was designed at the Communications Research Centre Canada was simulated and measured to validate the software. The array was series fed by a microstrip line. The dimensions of the array are shown in Figure 3.6. The simulated and measured results are compared in Figures 3.7-3.8.

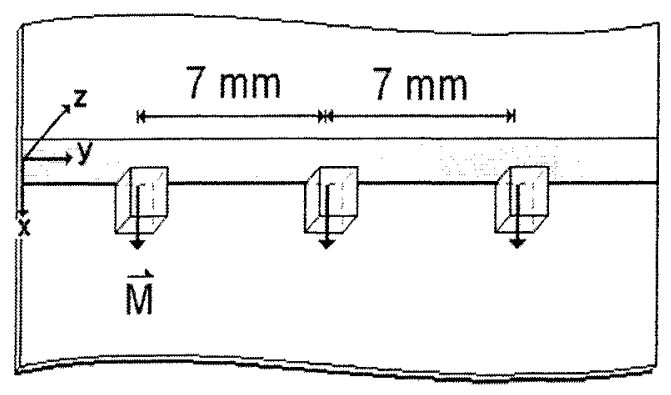

(a) Array dimensions

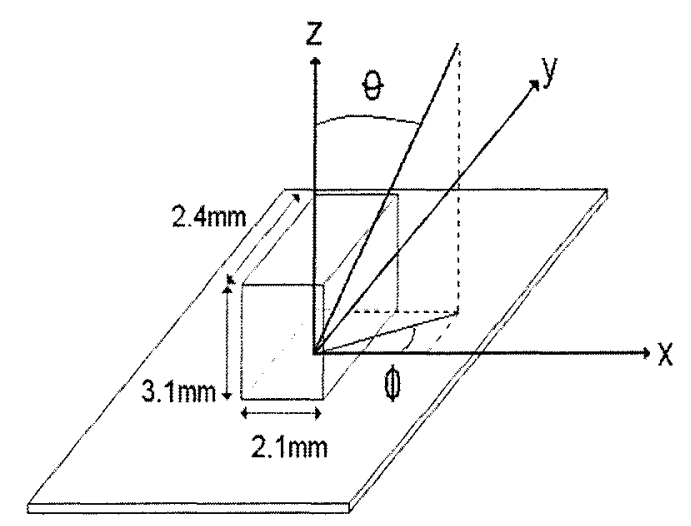

(b) DRA dimensions

Figure 3.6: Dimensions of microstrip fed DRA array for software validation

The microstrip line was connected to the receiver with k-connectors. Absorber material was placed near the connectors to reduce the effect of the connectors on the radiation pattern. 


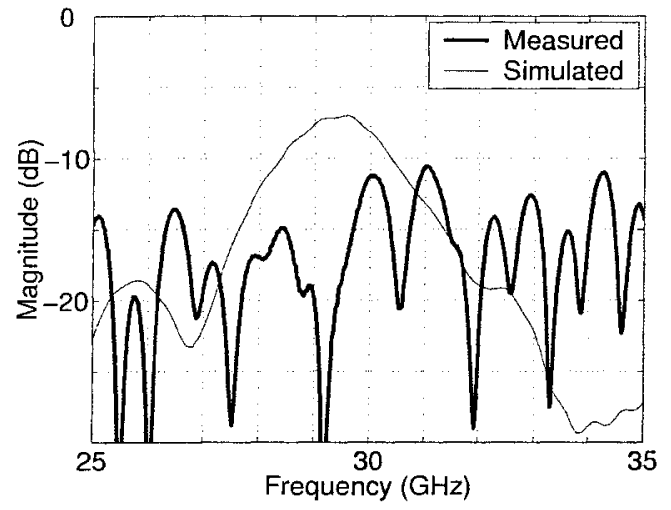

(a) $s_{11}$

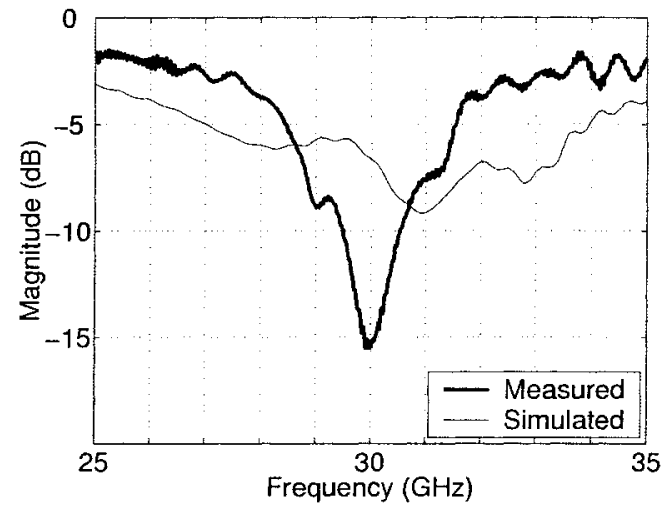

(b) $s_{21}$

Figure 3.7: S-parameters for microstrip fed DRA array

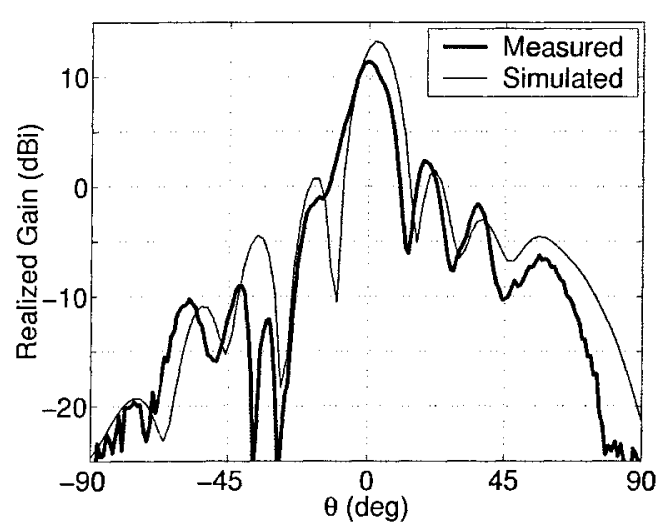

(a) $E_{\phi}$ in the H-plane $\left(\phi=90^{\circ}\right)$

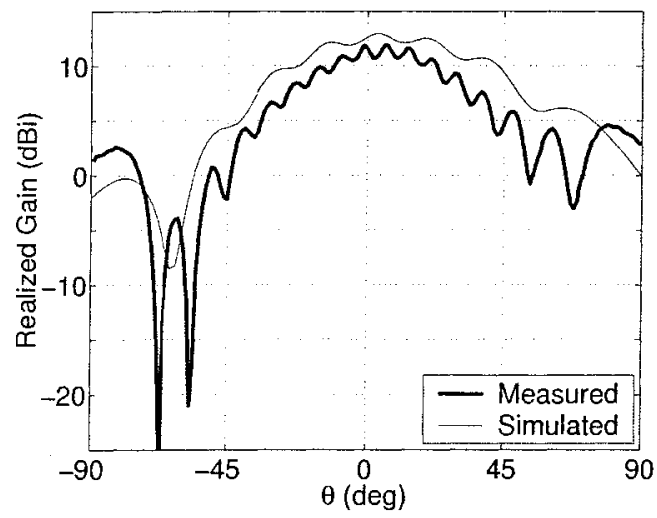

(c) $E_{\theta}$ in the E-plane $\left(\phi=0^{\circ}\right)$

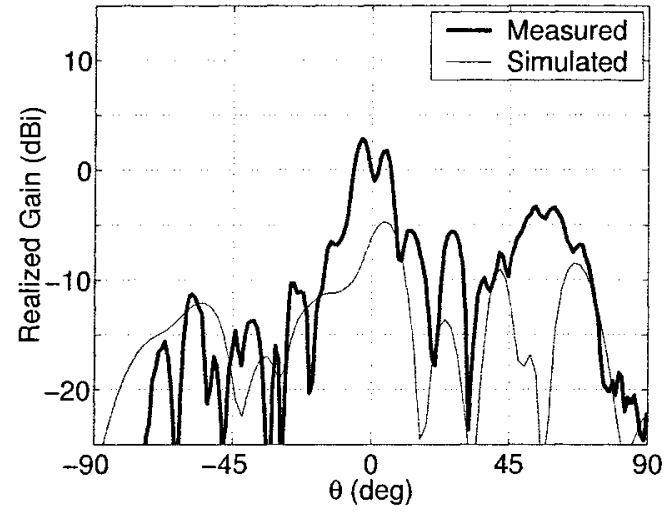

(b) $E_{\theta}$ in the H-plane $\left(\phi=90^{\circ}\right)$

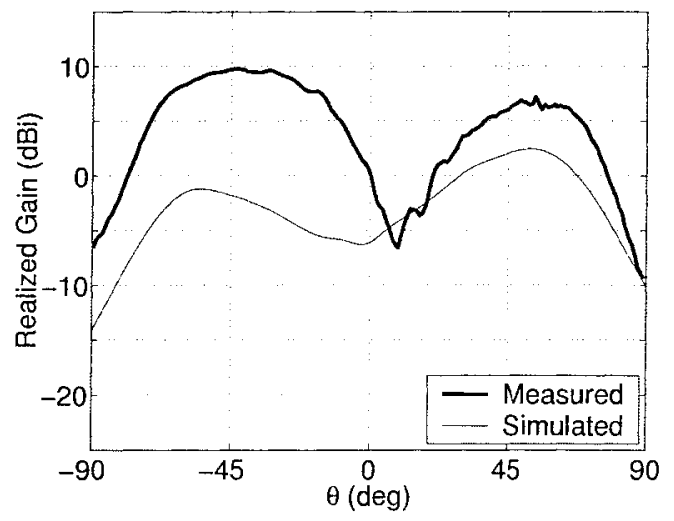

(d) $E_{\phi}$ in the E-plane $\left(\phi=0^{\circ}\right)$

Figure 3.8: Radiation patterns at $30 \mathrm{GHz}$ for microstrip fed DRA array 
The s-parameter results in Figure 3.7 show some disagreement between the simulated and measured results. The return loss for the measured result does not show a reflection larger than $-10 \mathrm{~dB}$. The simulated results show a strong reflection at approximately $29.5 \mathrm{GHz}$, which is due to the periodic loading of the DRA's. The measured results indicate that the element spacing is not exactly the same between the DRA's. The DRA's were placed by hand, therefore it was expected to have some variation in the element spacing. The simulated $s_{21}$ result shows that the DRA's are resonant around $31 \mathrm{GHz}$, but the measured show resonance at $30 \mathrm{GHz}$. This discrepencey could be due to the permittivity of the substrate or DRA's not being equal to the values used in the simulation.

Lossy materials were not introduced in simulation due to a lack of confidence in the software results when using finite conductivty. The loss of the connectors was also not accounted for in the simulation results. These losses are apparent by the difference in magnitude of the $s_{21}$ curves at resonance, and the difference between the maximum gain curves in Figures 3.8(a) and 3.8(c). The loss of the microstrip line was analytically calculated to be $0.9 \mathrm{~dB}$ at $30 \mathrm{GHz}$ over its $15 \mathrm{~cm}$ length. The line without the DRA's was measured to isolate the loss from the connectors. At $30 \mathrm{GHz}$ this loss was calculated to be approximately $1 \mathrm{~dB}$ per connector.

In Figure 3.8(a) the sidelobes and main beam have a similar shape for the measured and simulated results. The additional ripples in Figure 3.8(c) are due to the difference in the size of the actual ground plane and that used in the simulation. There is significant error in the simulated cross-polarization 
patterns, as shown in Figures 3.8(b) and 3.8(d). The cross-polarization is mainly due to the excitation of higher order modes in the DRA's. The location of the DRA's with respect to the microstrip line has a large impact on how the DRA is excited. Due to the primitive method used to position the DRA's, the position of each DRA with respect to the microstrip line is slightly different. In the simulation each DRA was placed perfectly flush to the line, but for the actual array there were small gaps between the line and some of the DRA's, also some overlap for other DRA's.

From these results, Empire has proven to be useful in predicting the copolarized radiation patterns of DRA arrays. The discrepancy between the remaining measurements highlight the sensitivity of the results with respect to the manufacturing process.

\subsection{Surface-Wave Launcher for the DIG}

To launch energy into a DIG over a broad bandwidth, a smooth transition is required that matches the dominant electric and magnetic field components of the DIG to those of the launcher. A variety of these transitions have been designed in the past, but all have drawbacks when considered for this work. Some of the designs have inherent fabrication complexities that make them unattractive. The transitions must be compatible with the test equipment's coaxial cables. The cables used were k-cables, which were selected based on the operating frequency of $30 \mathrm{GHz}$. 


\subsubsection{Launcher Survey}

This section discusses some of the transition designs for DIG's that have been reported in the literature. These transitions are shown in Figure 3.9. A new transition is presented, which addresses issues that are specific to this work.

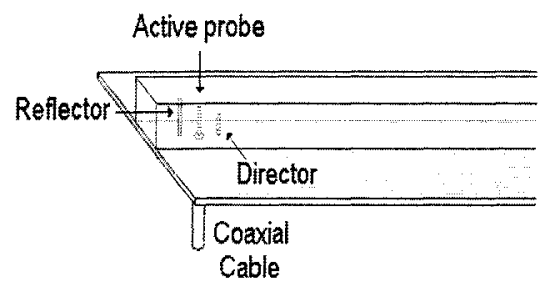

(a) Array of internal probes

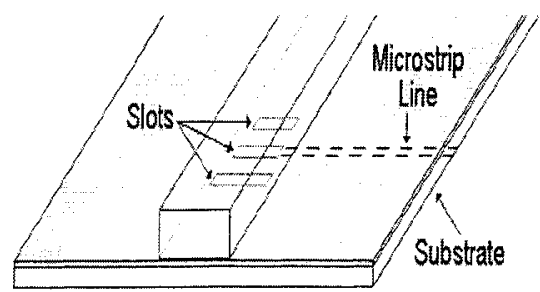

(c) Slot coupled microstrip

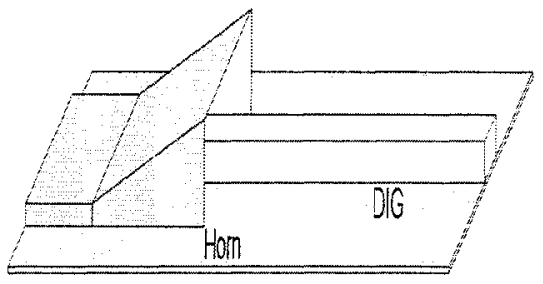

(b) Waveguide and horn

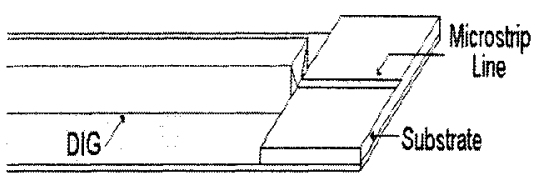

(d) Bent microstrip

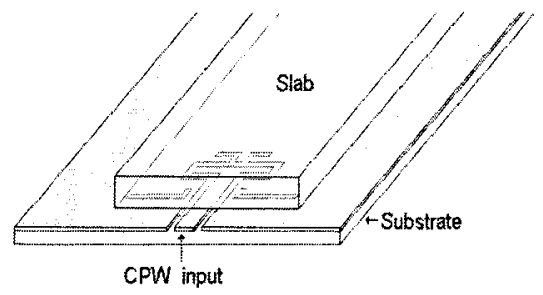

(e) CPW slab launcher

Figure 3.9: DIG transitions

\section{Direct probe feed}

The fields near the probe extruding out of the ground plane have similar components to those of the fundamental mode in the DIG. The first loca- 
tion for the probe that was considered was inside of the DIG. The probe is omni-directional, therefore a reactive component was required to maximize the energy in one direction. This was accomplished by using an array of probes in a Yagi-Uda array [33], shown in Figure 3.9(a). Having the probes inside the DIG makes fabrication increasingly complex as the width of the DIG becomes the same order of magnitude as the diameter of the probe, as is the case in this thesis work.

A different method to feed the DIG directly with a probe is to put the probe near one end of the DIG, but external to it. For a specific probe height the DIG will be acceptably matched to the probe resulting in some of the energy going into the guide. The probe is omni-directional, but with a DIG butted against it the fields will concentrate more towards the DIG. A method to concentrate more fields in the direction of the DIG is by moving the probe away from it's centre position in the hole in the ground plane, and towards the DIG. This feed method is shown in Figure 3.10.

A thick plate was used as the ground plane. A k-cable sized hole was milled from the bottom of the plate, but did not penetrate all of the way through. A larger hole was milled from the top that was slightly offset in the direction away from the DIG. This configuration resulted in a higher concentration of fields on one side of the probe, thereby transferring more power into the DIG than for the case of a single hole with the probe centred. The insertion loss corresponding to Figure 3.10 with and without the holes being offset is shown in Figure 3.11. To determine the insertion loss both ends of the DIG had 
identical coaxial transitions. The DIG used for these results corresponds to case $i$ in Table 3.1 .

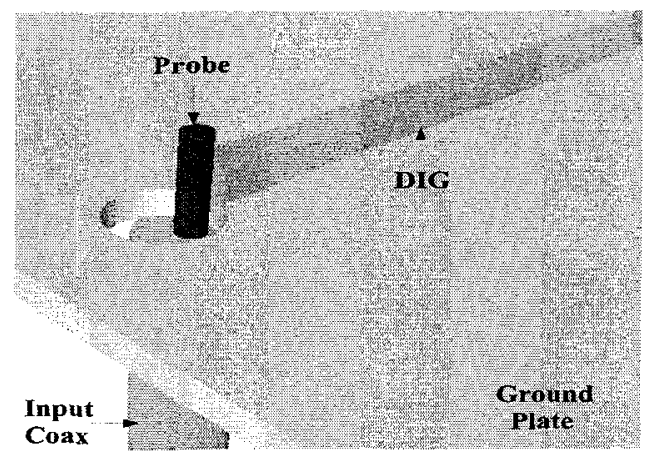

(a) 3-D Image

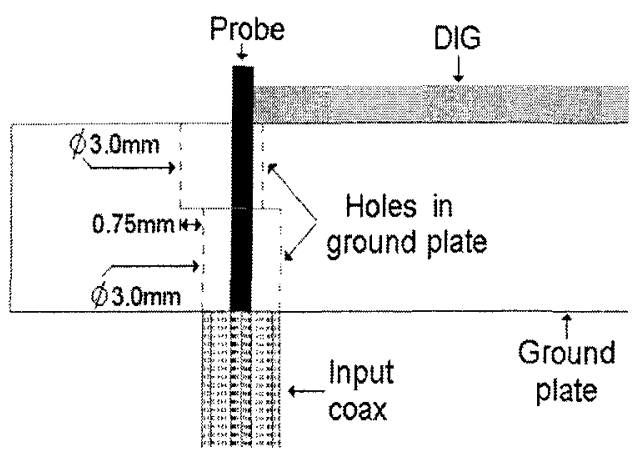

(b) Cross-section of transition (dimensions correspond to case $i$ of Table 3.1

Figure 3.10: New coaxial cable to DIG transition

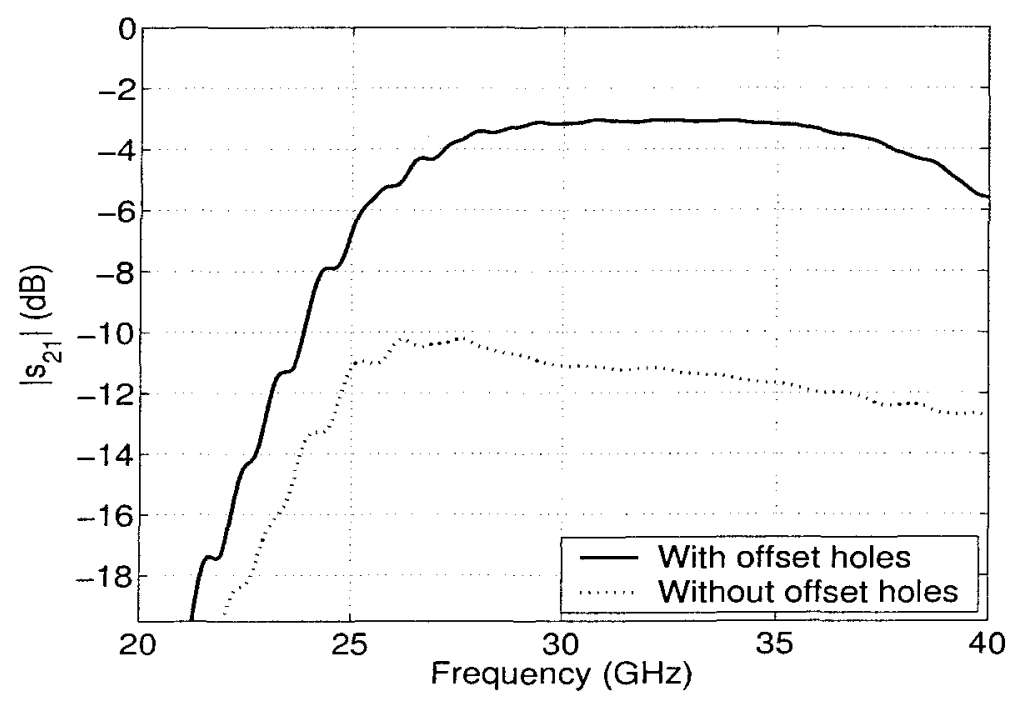

Figure 3.11: Insertion loss for setup in Figure 3.10

Each design parameter controls a certain aspect of the transition. The upper hole radius was used to control the concentration of fields towards one edge of the hole. The field concentration should match the mode profile of $E_{11}^{z}$ mode of the DIG. As this radius decreases, the energy spreads more around the 
edges of the hole, but when it increases the energy concentrates more between the probe and the nearest point to the edge of the hole. The offset between the two holes also effects the field concentration, but can cause an impedance mismatch. The probe height is adjusted to cancel the reactive component of the input impedance.

\section{Metal rectangular waveguide}

An early solution was to taper the image guide into a pyramidal horn, fed by a rectangular waveguide, shown in Figure 3.9(b) [22]. This approach boasted very good results over relatively large bandwidths [34]. All of the power launched in the guide travelled in one direction and there were many design variables to tune the device. A modification made to this design was to remove the horn and directly feed the tapered DIG with a rectangular waveguide [35]. The drawbacks of these transitions were the bulkiness and fabrication complexity associated with building a horn and rectangular waveguide components in the EHF band.

\section{Microstrip slot-coupled}

To use a slot-coupled feed architecture, the DIG is mounted on a ground plane, which is mounted on a substrate. A microstrip line is etched on the bottom of this substrate and the slots are etched out of the ground plane. There are various slot architectures that have be used $[36,37,38,39,40]$. One 
type is a Yagi-Uda array of slots, which launches the majority of the energy in one direction inside the DIG [37], shown in Figure 3.9(c). These slots were conceptualized to be fed with a microstrip line but the authors used a tapered dielectric waveguide to excite the centre slot to improve the coupling. A drawback of this design is that as the frequency increases, more energy will couple into surface-waves in the substrate.

\section{Flared microstrip on top of ramped DIG}

Two microstrip-to-DIG transitions were reported in [41] that incorporated a microstrip feed on the same side of the ground plane as the DIG. The first design was for small aspect ratios, and is shown in Figure 3.9(d). This design consisted of a microstrip line on a substrate, which is directly connected to a flared microstrip patch that is placed on one end of the DIG. The second design for larger aspect ratios included a ramped DIG between the substrate and main DIG. As the microstrip reaches closer to the top of the ramp it flares out to match the field components of the $E_{11}^{z}$ mode. The paper reported on the frequency range of 11 to $17 \mathrm{GHz}$. Within this frequency range a bandwidth of $26 \%$ was achieved defined by an insertion loss per transition better than 2 $\mathrm{dB}$ and a return loss better than $10 \mathrm{~dB}$. The second design resulted in a larger bandwidth over which the insertion loss per transition was better than $1.5 \mathrm{~dB}$.

The first design was attempted at $30 \mathrm{GHz}$ for one of the DIG's used in this work, but the abrupt transition resulted in unacceptable radiation losses. The second design had inherent fabrication complexities, and was found to be in- 
efficient to simulate using Empire. This simulator meshes the 3-D geometries into rectangular boxes, therefore ramped geometries must have dense meshing to capture the actual shape. For this transition the microstrip traces on the ramp caused the required meshing to be very dense just to ensure continuity along the microstrip line.

\section{Coplanar waveguide}

Coplanar waveguide (CPW) technology offers the advantage of a low profile transmission line integrated onto the ground plane. Figure 3.9(e) shows a configuration used for launching a surface-wave onto a dielectric slab [42]. A simple CPW can be used as a feed by using an open-ended slot. This configuration can be used to match the $\mathrm{H}$-fields of the bound mode in the DIG. The open-ended slot behaves similarly to an $x$-directed magnetic current, and the fundamental mode of the DIG has a large $H_{x}$ component at the interface with the ground plane. For loosely bound DIG's most of the energy does not travel within the DIG. This type of transition launches the energy directly into the DIG, therefore it would be an inefficient launcher.

\section{Survey Results}

One advantage of the DRA array is the low-profile characteristic, therefore the feed for the DIG should not tower above the DIG. For this reason waveguide components were not employed. A probe directly feeding the DIG was an 
attractive alternative since no other connectors, waveguide components, or microstrip traces would be required. The probe that is external to the DIG was chosen as the transition to use in this work.

\subsubsection{Results for Specific Launchers}

The insertion loss and radiation pattern of the input transition was characterized by simulations. The DIG is shown in Figure 3.12. The ground plane was omitted from this figure. This figure shows the losses within the system, which were used to calculate the loss per transition. The loss at each transition is due to reflection and radiation due to the discontinuity that each transition presents to the guided energy. Lossless materials were used in the simulations so that only the transitions contributed to the loss.

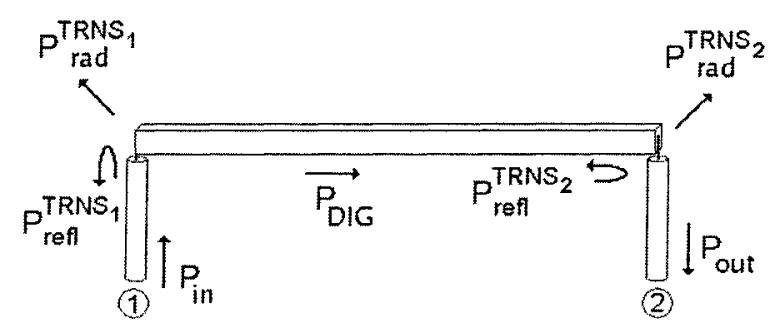

Figure 3.12: DIG without DRA's for characterization

The total loss of the system is given by the $s_{21}$ parameter. Both the input and output transitions were identical, hence by reciprocity the ratio of loss to power going into the transitions is equal. The total loss per transition $\left(\alpha_{\text {trans }}\right)$ is equal to half of the magnitude of $s_{21}$ in $\mathrm{dB}$. This relationship is shown in (3.15). The length of the DIG was $6 \lambda_{o}$, which was found to give approximately 
the same results as longer DIG's.

$$
\begin{gathered}
\alpha_{\text {trans }}=-10 \log _{10}\left(1-\frac{P_{\text {rad }}^{T R N S_{1}}+P_{\text {refl }}^{T R N S_{1}}}{P_{\text {in }}}\right) \\
=-10 \log _{10}\left(1-\frac{P_{\text {rad }}^{T R N S_{2}}+P_{\text {refl }}^{T R N S_{2}}}{P_{D I G}}\right) \\
=-\frac{s_{21}}{2} \quad[\mathrm{~dB} / \text { trans }]
\end{gathered}
$$

The loss per transition was further subdivided into radiation $\left(\alpha_{\text {rad }}\right)$ and reflection $\left(\alpha_{r e f l}\right)$ losses. The $s_{11}$ parameter was used to determine the reflection due solely to the first transition. The FDTD simulation was run for a specific number of time steps so that the portion of the excitation pulse that reflected back from the second transition was gated out.

For each of the image guides defined in Table 3.1 a transition was designed. The resulting losses from each the transition are summarized in Table 3.2. The s-parameters for case $i$ are plotted in Figure 3.13. The radiation pattern in the principle planes due solely to the first transition are shown in Figure 3.14. With DRA's present, they will be coupling most of the energy out of the DIG therefore the radiation from the second transition will be less significant. 
Table 3.2: Transition losses at $30 \mathrm{GHz}$

\begin{tabular}{|c|c|c|c|c|}
\hline Case & $\begin{array}{r}\left|s_{11}\right| \\
(\mathrm{dB})\end{array}$ & $\begin{array}{c}\left|s_{21}\right| \\
(\mathrm{dB})\end{array}$ & $\begin{array}{c}\alpha_{\text {rad }} \\
(\mathrm{dB} / \text { trans })\end{array}$ & $\begin{array}{c}\alpha_{\text {refl }} \\
(\mathrm{dB} / \text { trans })\end{array}$ \\
\hline \hline$i$ & -10.5 & -3.15 & -1.175 & -0.4 \\
\hline$i i$ & -12 & -2.50 & -0.95 & -0.3 \\
\hline$i i i$ & -27 & -3.75 & -1.875 & 0 \\
\hline$i v$ & -16 & -2.14 & -0.27 & -0.11 \\
\hline
\end{tabular}

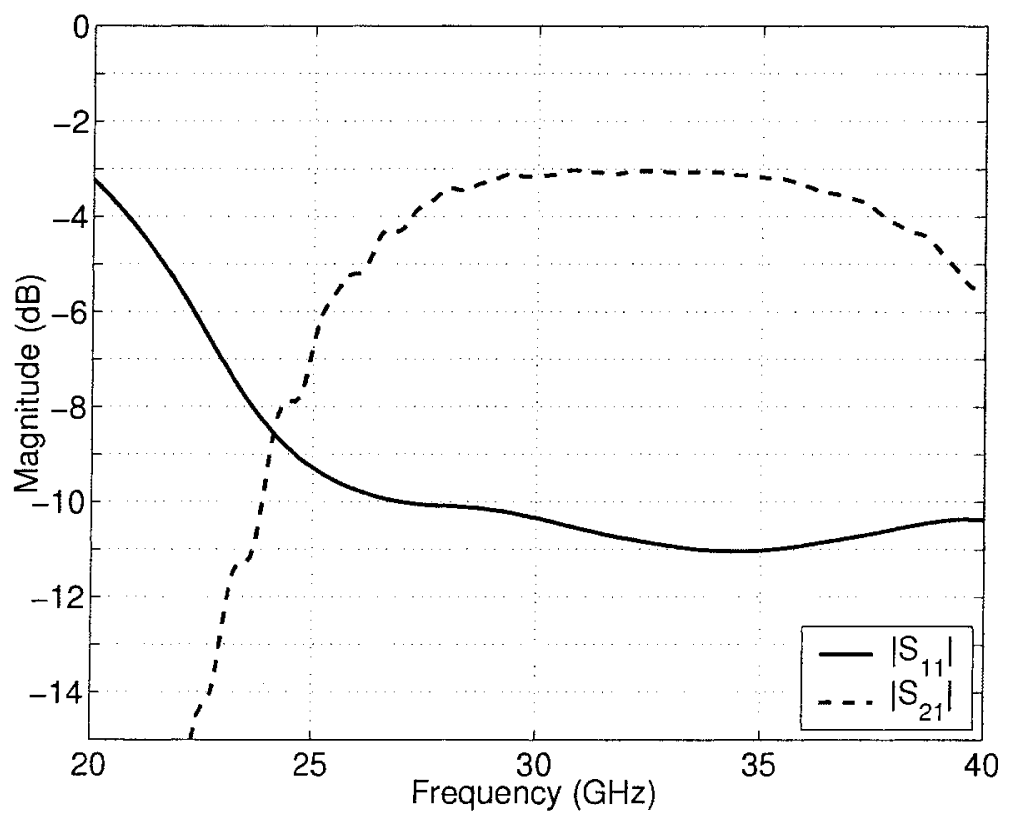

Figure 3.13: S-parameters for DIG with two identical transitions (case $i$ ) 


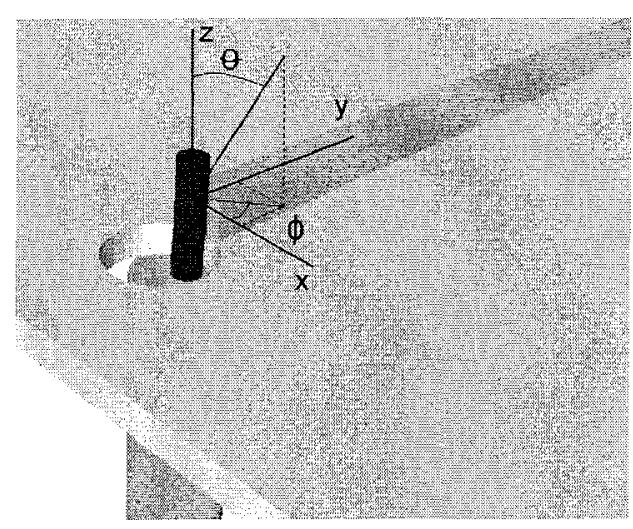

(a) Feed with $\theta$ and $\phi$

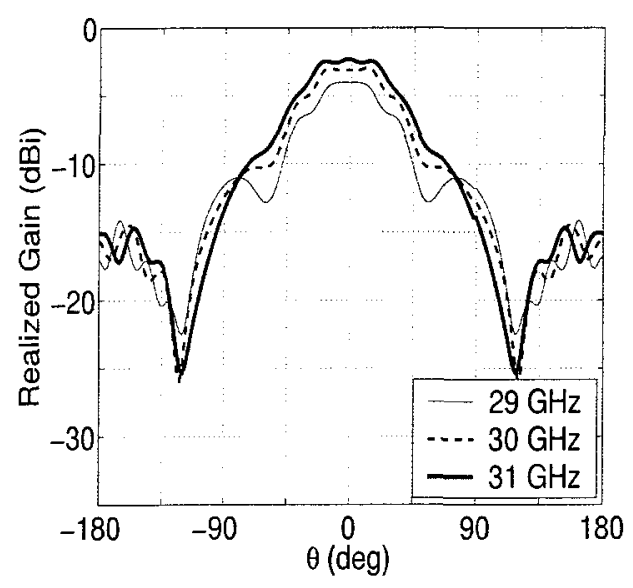

(c) $E_{\phi}$ in $\phi=0^{\circ}$ plane

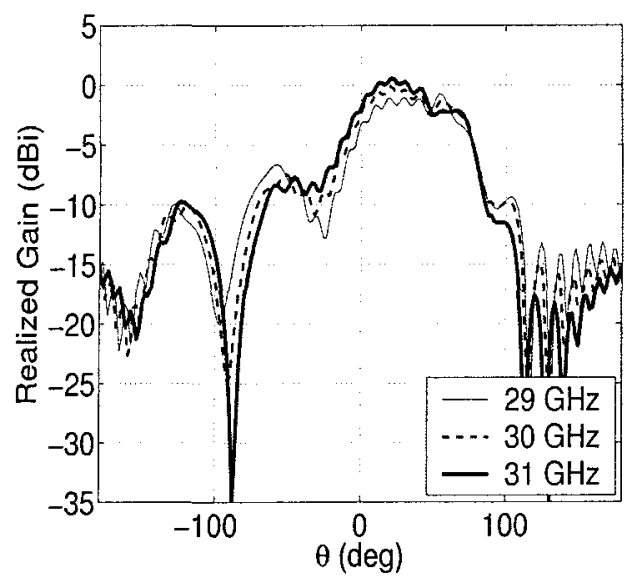

(b) $E_{\theta}$ in $\phi=90^{\circ}$ plane

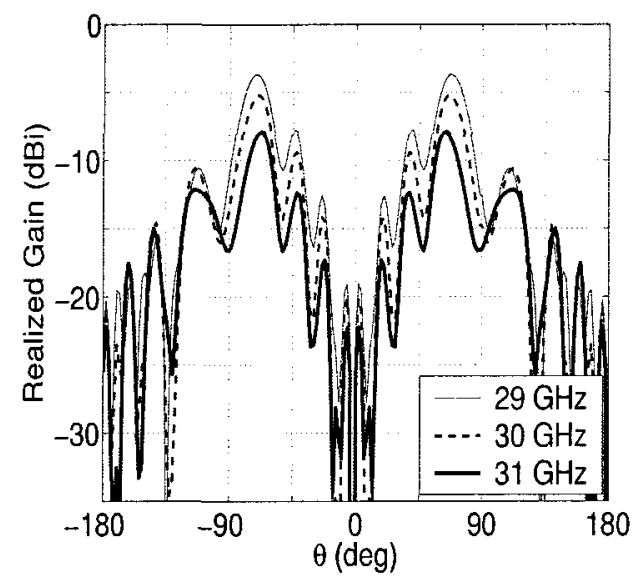

(d) $E_{\theta}$ in $\phi=0^{\circ}$ plane

Figure 3.14: Gain of the feeding port (case $i$ )

\section{Chapter Summary}

A survey was conducted of several types of open waveguiding structures to use as the feed network for the array in this thesis work. The DIG was chosen, and the equations that relate the DIG characteristics to the dimensions and permittivity were presented. A transition was designed to connect a coaxial cable to a DIG. The transition was validated with simulations. 


\section{Chapter 4}

\section{The DRA Array Fed by the DIG}

The antenna studied in this work was an array of DRA's fed by a DIG. A specific phase distribution was achieved by basing the element spacing on the DIG's guided wavelength. A specific amplitude distribution was achieved by controlling the amount of power that coupled into each DRA. This control was accomplished by varying the distance between the DIG and each DRA. Coupled mode theory was used to approximate the amount of power coupled from the DIG to the DRA's as a function of this distance. Simulations validated the analytical results, and gave insight into other characteristics of the array. The presence of the DIG complicated the operation of the DRA. The loading shifted the resonance frequency upward, and deviated the radiation pattern away from that of a short magnetic dipole. 


\subsection{The History of DIG Fed Arrays}

In 1981 Birand and Gelsthorpe introduced the concept of placing dielectric radiating elements alongside an insular DIG to realize broadside radiation [5]. It was claimed that the elements could be separated from the guide by different distances to obtain a specific amplitude distribution. In the experiment all 12 elements were separated from the insular DIG by the same distance. This distance was very small since only a small amount of power coupled into each element. The radiation pattern, normalized by the maximum value, was reported in the array plane, but not the absolute value of the gain. The maximum sidelobe level in the array plane was $11.5 \mathrm{~dB}$ below the main beam. This paper was followed up by a patent, but without any further results [43]. This type of array has not been designed with a common amplitude distribution.

A similar array design was accomplished by replacing the dielectric resonators with microstrip patch elements [6], also in 1981. The authors discussed the possibility of designing for a specific amplitude distribution by varying the distances between the patches and the DIG. A higher order mode, namely the $E_{21}^{z}$ mode, was used in the guide to maximize the power coupled into the patches. Two different arrays were fabricated to operate at $14 \mathrm{GHz}$ and at $70 \mathrm{GHz}$. Similar to Birand and Gelsthorpe's design, all elements were located equidistant from the DIG. A patent was successfully filed for this design [44].

In 1995 a low permittivity DIG $\left(\epsilon_{r}=2.55\right)$ was used as a series feed for an array of 20 DRA's at $20 \mathrm{GHz}[7]$. The DRA's were made of a higher permittivity 
material $\left(\epsilon_{r}=10.8\right)$. The DRA's were placed directly against the DIG. The element spacing resulted in broadside radiation, where a gain of $11.6 \mathrm{dBi}$ was measured (at $20.4 \mathrm{GHz}$ ). A maximum gain of $13.2 \mathrm{dBi}$ was measured at 21.8 $\mathrm{GHz}$, where the main beam had scanned off broadside by $6^{\circ}$. In the work for this paper the $T E_{1 \delta 1}^{y}$ radiating mode of the DRA was excited.

\subsection{Coupling Between the DIG and the DRA - Analytical}

The fundamental mode of the DIG in Figure 4.1(a) is the $E_{11}^{z}$ mode. This mode characteristically has dominant $E_{z}, E_{y}$, and $H_{x}$ components. The DRA has two main radiating magnetic dipole modes that can be excited by the DIG, namely the $T E_{1 \delta 1}^{y}$ and the $T E_{\delta 11}^{x}$. The latter mode has a large $H_{x}$ component, therefore overlaps well with the $E_{11}^{z}$ mode of the DIG. The electric field inside the DRA operating in this magnetic dipole mode is shown in Figure 4.1(b). Two methods were investigated to approximate the amount of power coupled from the DIG into the DRA's:

- equivalent circuit model

- coupled mode theory 


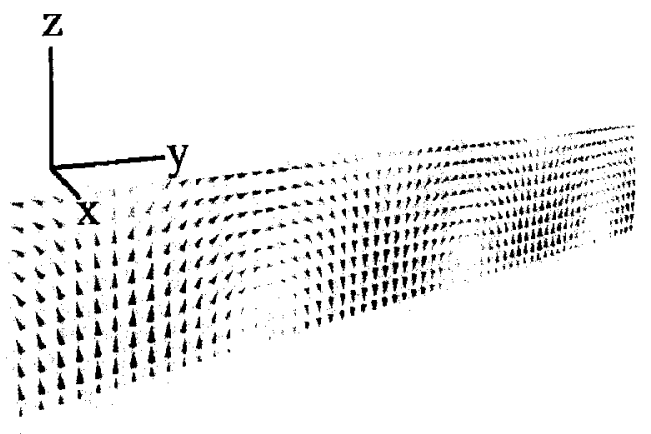

(a) $E_{11}^{z}$ of DIG

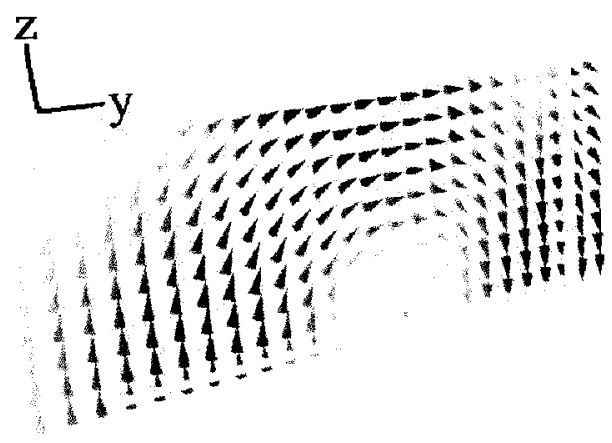

(b) $T E_{\delta 11}^{x}$ of DRA

Figure 4.1: E-field of fundamental modes (ground plane omitted)

\subsubsection{Equivalent Circuit Model}

A common method of analyzing a resonator in proximity to a transmission line is by modelling the problem with an equivalent circuit. Figure 4.2 shows this circuit where $L_{D R A}, C_{D R A}$ and $R_{D R A}$ correspond to the inductance, capacitance and resistance of the DRA, and $L_{T L}, C_{T L}$ and $R_{T L}$ correspond to those of the transmission line. The parameter $L_{M}$ is the mutual inductance between the transmission line and the DRA. The voltage generator parameters are $v_{g}$ and $R_{g}$, and the load impedance is $Z_{\text {Load }}$. This problem has been solved for

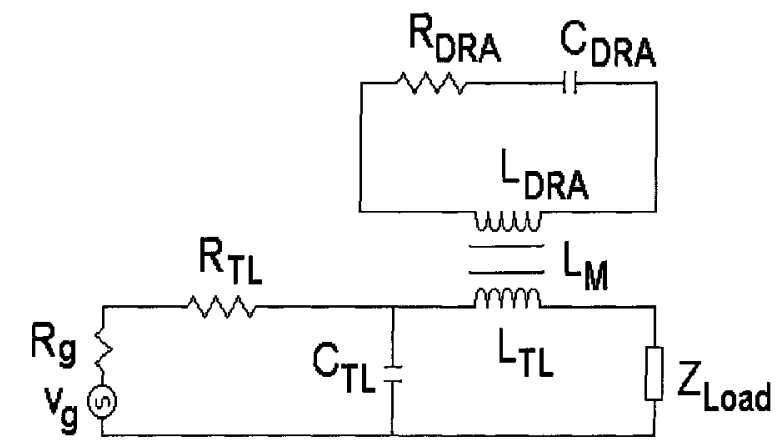

Figure 4.2: Equivalent circuit for transmission line and resonator 
the DIG and dielectric resonator [45]. The primary drawback of applying this method to a DRA instead of a dielectric resonator is that the DRA parameters $L_{D R A}, C_{D R A}$ and $R_{D R A}$ are calculated with less accuracy. The mutual inductance term $\left(L_{M}\right)$ varies as the distance between the DIG and the resonator varies, therefore contains the important information required to determine the coupling as a function of this distance. This term requires the knowledge of the magnetic fields of the transmission line and the DRA. The dielectric waveguide model can be used to calculated the fields of the DRA [10], but they will contain significant error due to the application of Marcatili's method [17]. The problems with this method were discussed in section 3.2. A rigorous modematching method was applied to the DIG cross-section to determine the fields and propagation constants. The field results contained significant error in the vicinity of the corners of the DIG. The propagation constants did not have significant error, therefore a method using the propagation constants instead of the magnetic fields would be desirable.

\subsubsection{Coupled Mode Theory}

Coupled mode theory (CMT) was used to approximate the coupling between the DRA and DIG as a function of the spacing between them [46]. The advantage of CMT was that the fields were not required. Instead, the propagation constants of an equivalent directional coupler were used to approximate the coupling. 


\section{Introduction to the directional coupler}

Two parallel DIG's form a directional coupler. Two different DIG couplers are shown in Figure 4.3 with their port numbers circled. An ideal 0 -dB coupler transfers all of the energy from one DIG to the other $\left(s_{41}=0 \mathrm{~dB}\right)$. An ideal 3$\mathrm{dB}$ coupler transfers half of the input energy to the coupled DIG while keeping the other half in the feeding DIG $\left(s_{21}=s_{41}=-3 \mathrm{~dB}\right)$. The physical difference between these couplers is the length of the coupled guide (top guide) that is within a close proximity to the feeding guide (bottom guide). The DIG's in Figure 4.3 have identical dimensions in the cross-section, and are both separated by the same distance $(s)$. The amount of power transferred to the coupled guide is controlled by varying the spacing between the guides and the length of the coupled guide within the coupling region. A common method of analysis for this type of directional coupler is based on CMT.

\section{Introduction to CMT for the directional coupler}

Marcatili investigated rectangular dielectric waveguides for use as directional couplers for integrated optics [17]. The main parameter of interest for a directional coupler is the coupling length $\left(L_{c}\right)$. This parameter is defined as half of the distance between maxima along the length of one of the guides. For couplers constructed of identical DIG's, the coupling length refers to the length of coupler required for $100 \%$ of the energy in one of the guides to transfer to the other, as shown in Figure 4.4. 


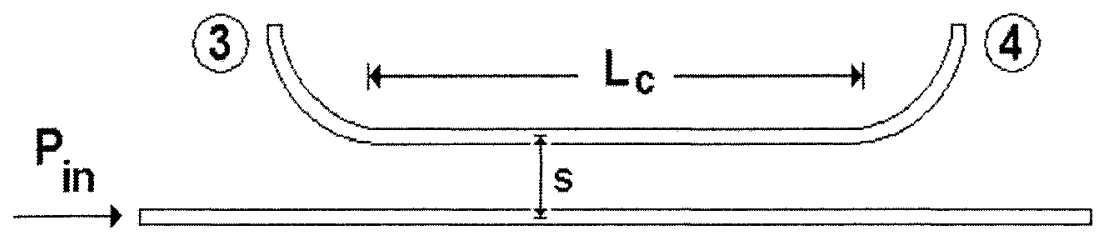

(1)

(2)

(a) 0-dB Coupler

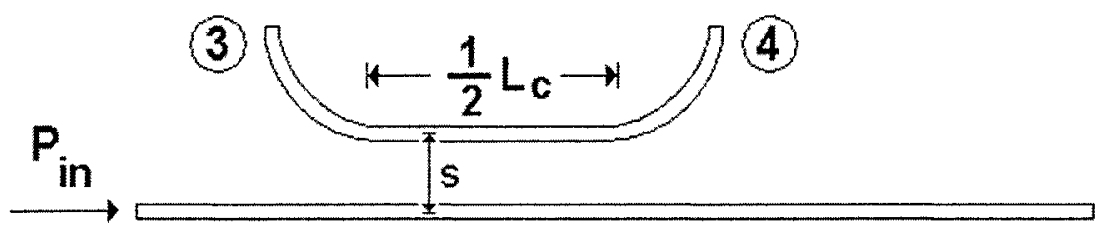

(1)

(b) 3-dB Coupler

Figure 4.3: Directional couplers constructed from DIG's

The amplitude of the electric fields in each of the guides varies sinusoidally along the length of the coupler. The equation governing the ratio of power in the coupled guide $\left(P_{2}\right)$ to the input power of the coupler $\left(P_{i n}\right)$ is given by (4.1). This function is plotted in Figure 4.5, where the variable $y$ represents the distance along the axial direction of the coupler.

$$
\frac{P_{2}}{P_{i n}}=\sin ^{2}\left(\frac{y}{L_{c}} \frac{\pi}{2}\right)
$$

To apply CMT, it was assumed that the energy in the directional coupler was propagating in its two lowest order modes, namely the $E_{11 e}^{z}$ and $E_{110}^{z}$ modes. The amplitude profile of these even and odd modes is shown in Figure 4.6. These modes have propagation constants $\beta_{e}$ and $\beta_{o}$ that depend on the DIG 


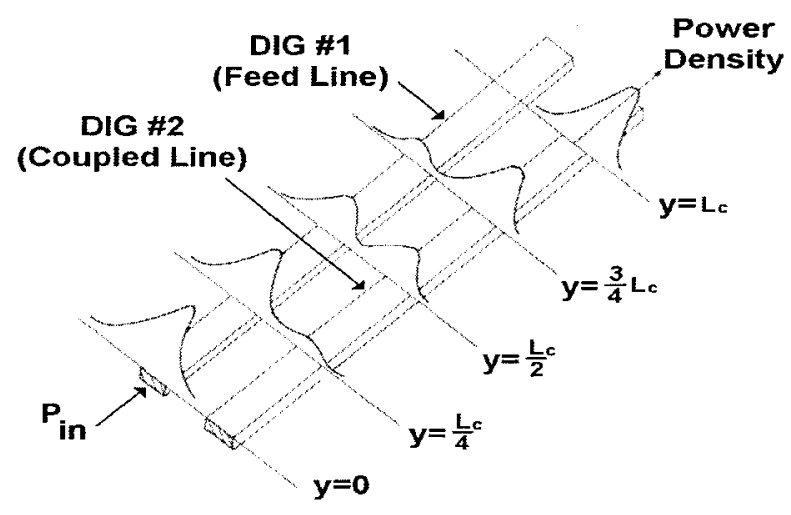

Figure 4.4: Power density along coupler

geometry $(h$ and $w)$, permittivity $\left(\epsilon_{r}\right)$ and spacing between the DIG's $(s)$.

At the input of the directional coupler all of the power is guided by the feed line (DIG \#1). The summation of the mode profiles from Figure 4.6 results in a mode profile for the coupler that is similar to the initial condition at the input where all of the power is in one DIG. The summation of these modes is shown in Figure 4.7(a). Qualitatively, this mode summation shows that the energy is propagating equally in the $E_{11 e}^{z}$ and $E_{11 o}^{z}$ modes, and at the input these modes are in phase with respect to the mode profiles in Figure 4.6.

After moving along the axial direction of the coupler for a distance $L_{c}$ all of the power has been transferred to the coupled line (DIG \#2). At this point, the subtraction of the mode profiles from Figure 4.6 results in a mode profile for the coupler that is similar to the situation at $y=L_{c}$ where all of the power is in the coupled DIG. The subtraction of these modes is shown in Figure 4.7(b). Subtracting the mode profiles is equivalent to them being $180^{\circ}$ out of phase with respect to the mode profiles in Figure 4.6. 


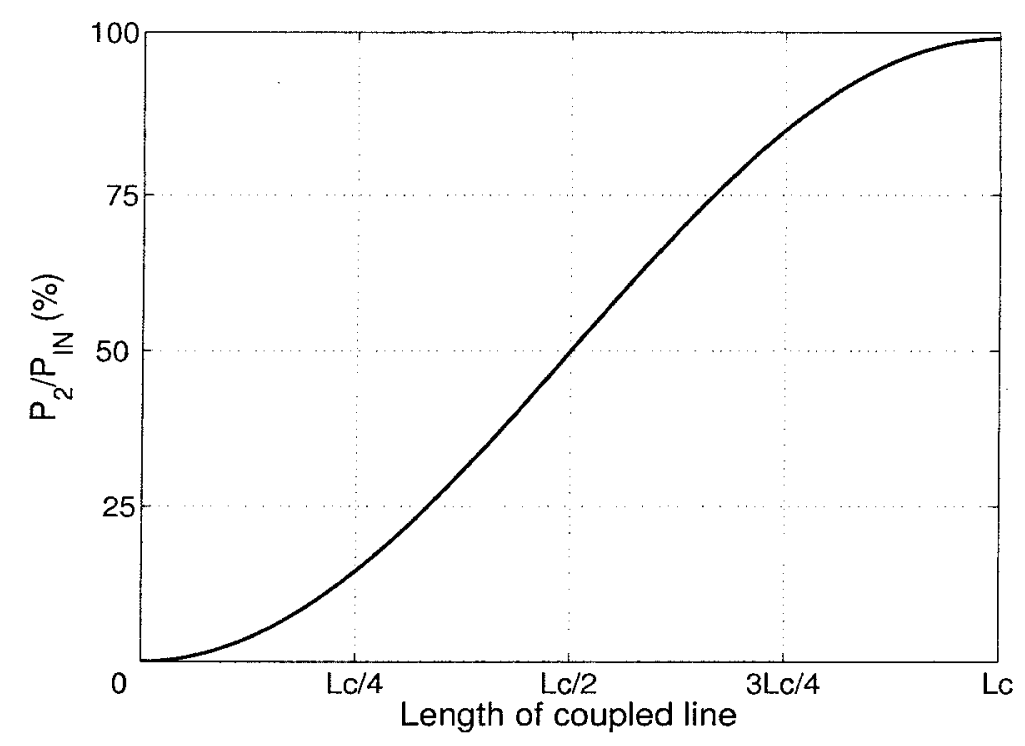

Figure 4.5: Power along coupler

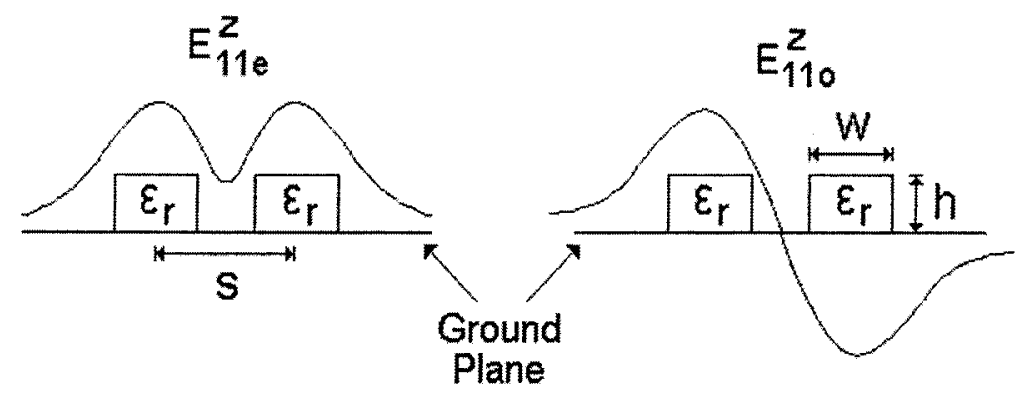

Figure 4.6: $E_{z}$ component of lowest order even and odd modes

As $s$ becomes very small the mode profiles change significantly from those in Figure 4.6. Furthermore, the odd mode has a cut-off frequency which increases as $s$ decreases. These results mean that the accuracy of CMT deteriorates for closely spaced DIG's. 

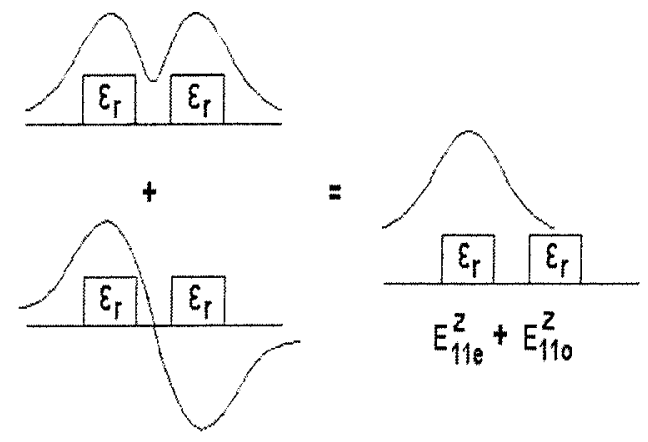

(a) Modes in phase
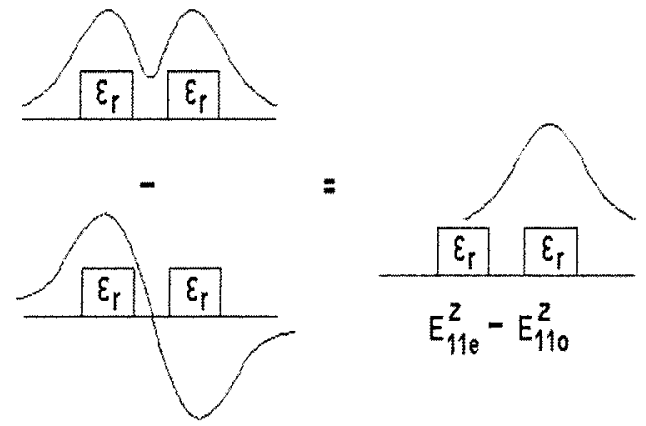

(b) Modes $180^{\circ}$ out of phase

Figure 4.7: Summation of even and odd modes

The coupling length is calculated from the propagation constants [47]:

$$
L_{c}=\frac{\pi}{\beta_{e}-\beta_{o}}
$$

Equations (4.1) and (4.2) can be used to determine the properties of a DIG directional coupler including the variation of power along it's axial direction from only the propagation constants; $\beta_{e}$ and $\beta_{o}$. These values are the eigenvalues of the coupler's cross-section. The eigenfunctions that correspond to these eigenvalues are the mode profiles of the $E_{11 e}^{z}$ and $E_{110}^{z}$ modes.

Several analysis methods have been used to solve the eigenvalue problem for two parallel DIG's [17, 27, 29]. A rigorous mode-matching solution was used [29] to calculate $\beta_{e}$ and $\beta_{o}$ for different DIG geometries. The details of this method are contained in appendix A.

For a particular pair of DIG's, the propagation constants were calculated for different values of $s$. A typical relationship between $L_{c}$ and $s$ is show in Figure 
4.8. The DIG parameters that were used to obtain the results in Figure 4.8 correspond to case $i$ of Table 3.1 .

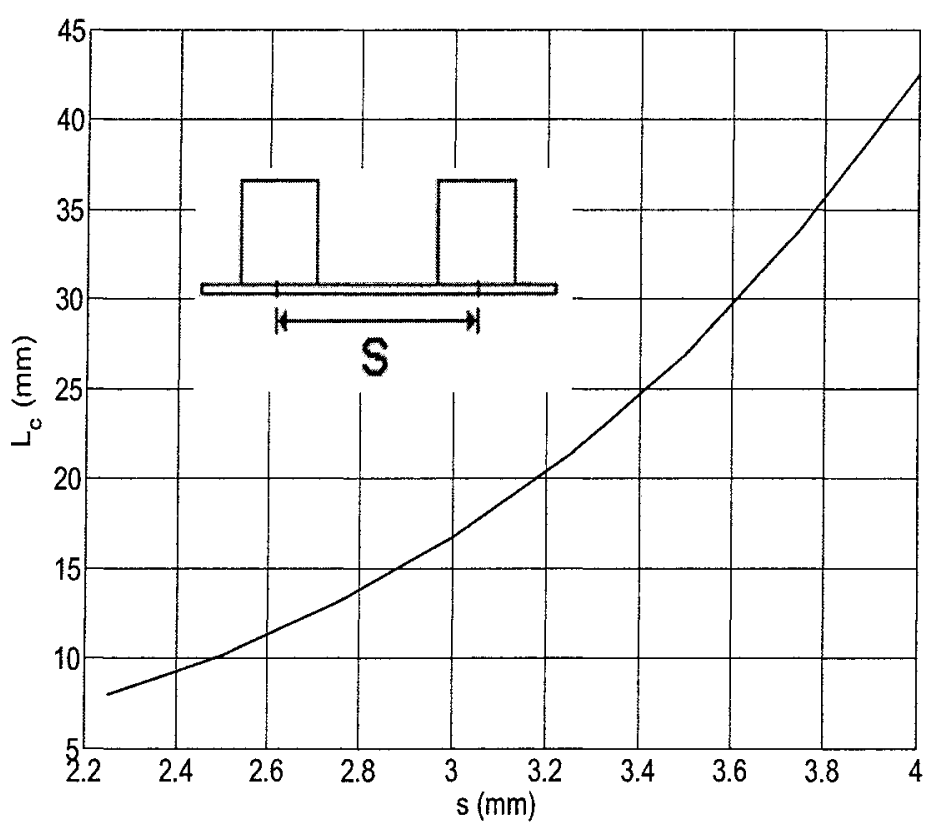

Figure 4.8: Typical coupling length curve

Figure 4.8 and (4.1) can be used to determine how much power transfers into the coupled guide for a specific coupler length and spacing.

If the two DIG's in the coupler are not identical then $L_{c}$ is calculated in the same manner, but the power will not be equally divided into the even and odd modes. This results in a coupler that cannot transfer $100 \%$ of its power between the guides. This type of coupler applied to the DIG fed DRA array is discussed in section 5.1.3. 


\section{CMT for the DIG with the DRA}

The DRA can be considered as a truncated DIG therefore a cross-section encompassing both the DIG and a DRA is identical to that of a directional coupler, as shown in Figure 4.9. CMT was applied to this cross-section to approximate the coupling properties between the DIG and DRA.

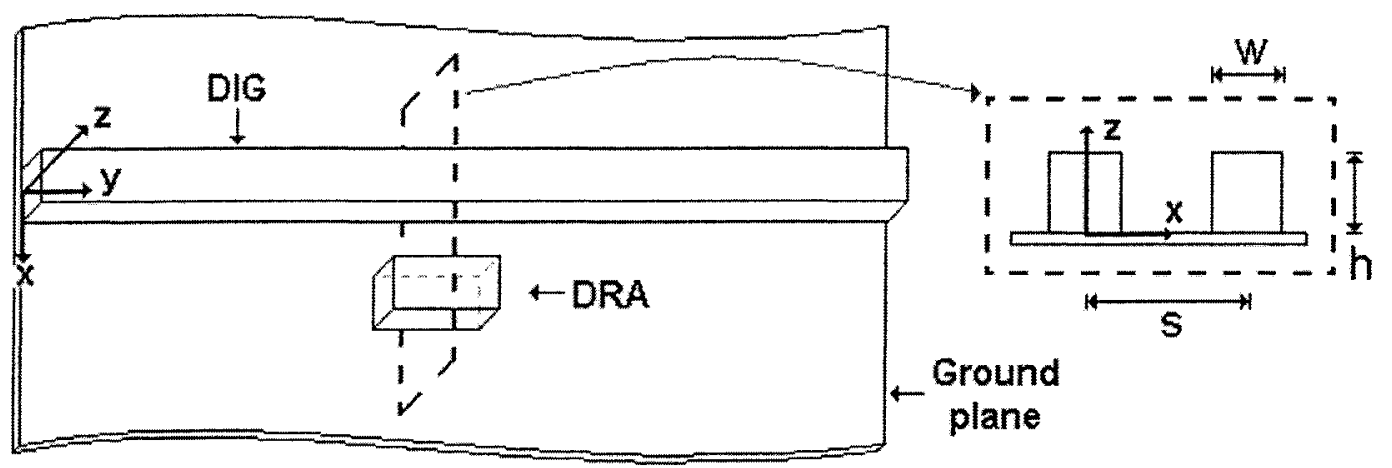

Figure 4.9: Cross-section of DRA near DIG with identical cross-sections

Two of the DRA's dimensions and its permittivity were made identical to the DIG to allow for the direct application of (4.1). Using this equation, for a DRA with a length of $L_{D R A}$, the power coupled into the DRA $\left(P_{D R A}\right)$ from the DIG $\left(P_{D I G}\right)$ is:

$$
\frac{P_{D R A}}{P_{D I G}} \approx \sin ^{2}\left(\frac{L_{D R A}}{L_{c}} \frac{\pi}{2}\right)
$$

Typically the length of the DRA is less than $L_{c}$. From (4.3), a long DRA will couple more energy out of the DIG than a shorter one. The DRA's length cannot be arbitrary since it must resonate at the operating frequency. The dielectric waveguide model (DWGM) is the common method of calculating 
the resonant frequency of a rectangular DRA [10]. Due to the significant inductive loading by the DIG on the DRA, the DWGM does not accurately predict the resonant frequency when the DRA is in close proximity to a DIG. The magnitude of this loading varies with the DRA-DIG spacing, therefore the length of DRA required for resonance is a function of $s$. As $s$ increases the error from the DWGM decreases.

In the vicinity of the DRA the energy is propagating in the $E_{11 e}^{z}$ and $E_{110}^{z}$ modes. As this energy reaches the end of the DRA, some of it is reflected back. This reflection is necessary for the DRA to be resonant and it sets up a standing wave inside the DRA. The fields in the DRA for the $T E_{\delta 11}^{x}$ mode are shown in Figure 4.1(b). The fields show half a cycle along the $y$-dimension. To get this half-cycle variation the length of the DRA must be half of the wavelength of the mode that the energy is propagating in. There are different reflection coefficients for the even and odd modes with regard to the discontinuity that the end of the DRA presents. The discontinuity is asymmetric, therefore reflects the odd mode more than the even mode. The standing wave in the DRA is due mostly to the reflection of the odd mode, therefore the length of the DRA must be approximately $1 / 2 \lambda_{110}$ for resonance. The resonant length was determined by simulations to validate this approximation. The comparison between the two results for each of the DIG's in Table 3.1 is shown in Figure 4.10.

The relationship between the DRA length and $s$ were combined to produce the relationship between the $s$ and the power coupled from the DIG into the 


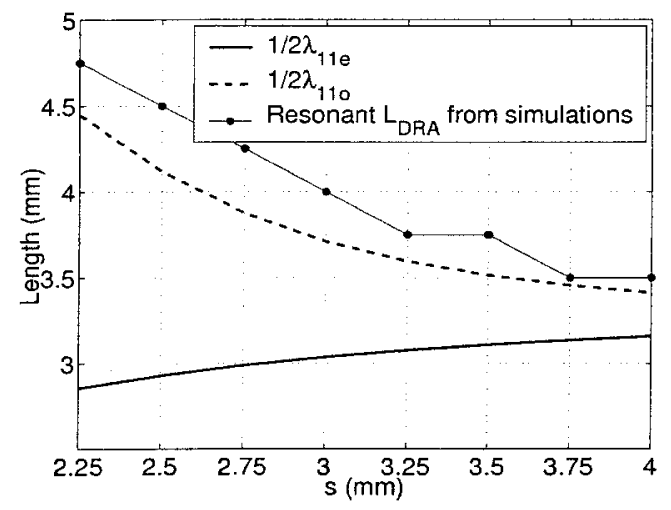

(a) Case $i$

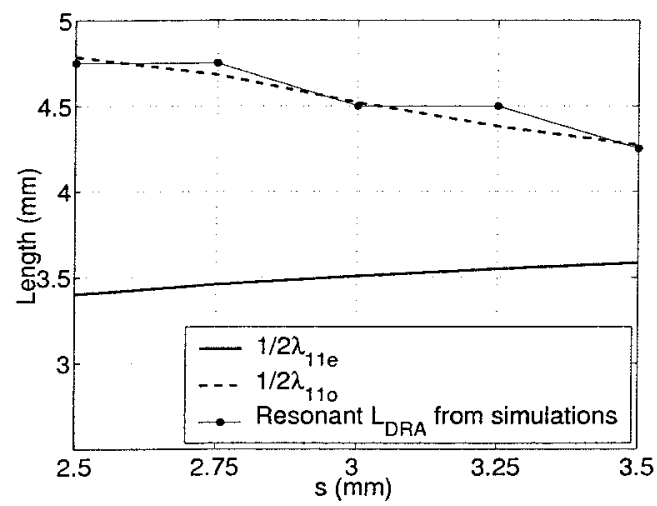

(c) Case $i i i$

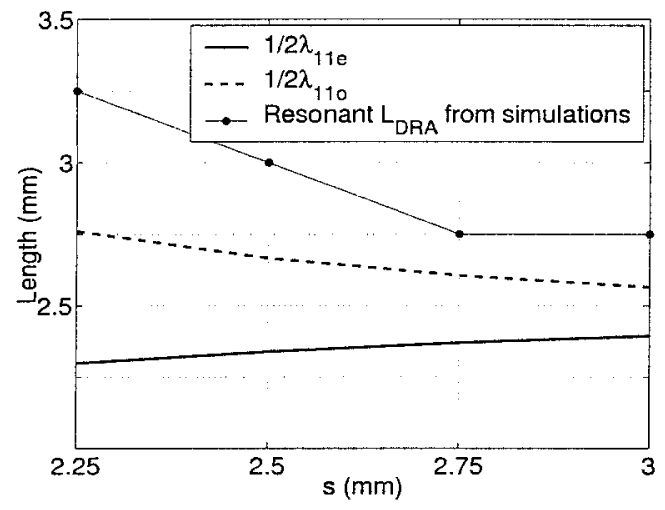

(b) Case $i i$

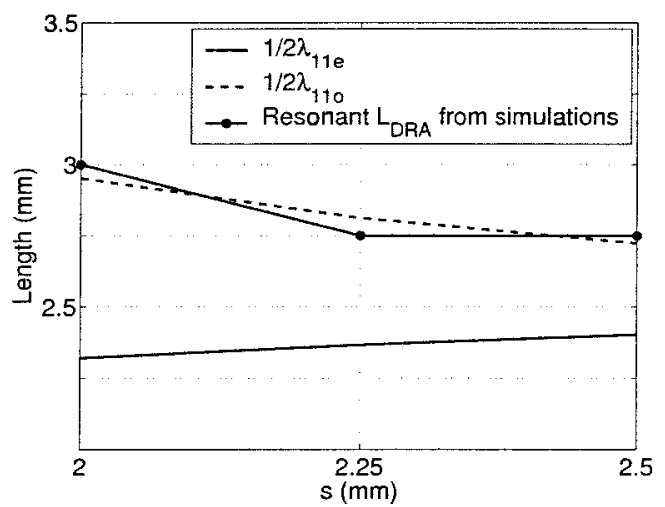

(d) Case $i v$

Figure 4.10: Comparison between mode wavelengths and simulated resonant length

DRA. This relationship is plotted in Figure 4.11. The DIG parameters used to create this figure correspond to case $i$ of Table 3.1. An antenna designer can use this graph to determine the value of $s$ required to couple a specific amount of power into a DRA. The designer must use that value of $s$ to calculated $1 / 2 \lambda_{11 \text { o }}$ to get the required DRA length.

From the required amplitude distribution of the array, the required ratio of power to couple into each DRA from the DIG can be calculated. In an array 


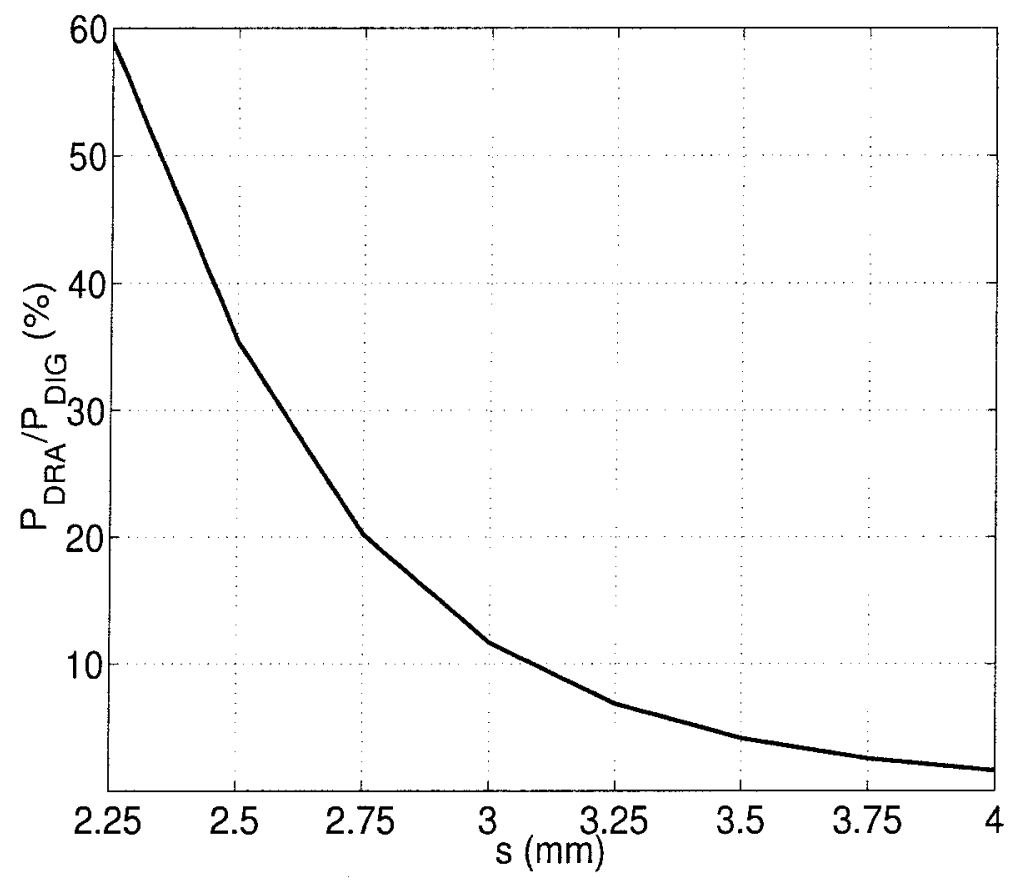

Figure 4.11: Power coupled from the DIG to a DRA

environment the power in the DIG at the location of each DRA is different. The power in the DIG at the point just before the $n^{\text {th }}$ DRA is represented by $P_{D I G_{n}}$. The other terms that acquired an $n$-subscript were the DRA length, DRA-DIG spacing and required power for the DRA $\left(L_{D R A_{n}}, s_{n}\right.$ and $P_{D R A_{n}}$, respectively).

To apply CMT for the case of a DIG-to-DRA instead of a DIG-to-DIG, it was assumed that the DRA's did not present large discontinuities to the mode on the DIG at the centre frequency, and each of the DRA's had a low quality factor. These assumptions translate into the DRA's not behaving like a resonator to the transmission line. If the assumptions were incorrect, then a large amount of energy would be reflected by the DRA. These assumptions 
were verified in section 4.3 by comparing the return loss with and without the DRA's present.

\subsection{Coupling Between the DIG and the DRA - Simulation}

The EM simulator used to validate the analytical approximation was Empire. This solver applied the FDTD method. The input port was excited with a user-defined pulse, which the solver then propagated through the structure. The number of time steps was user-defined.

\subsubsection{Test Setup}

The problem shown in Figure 4.12 was simulated with and without a DRA present. The $s_{21}$ was compared between the two cases. The difference between the $s_{21}$ values for both cases represented how much less power was transmitted through to the output port due to the presence of a DRA. The drop in power was attributed to radiation and return losses.

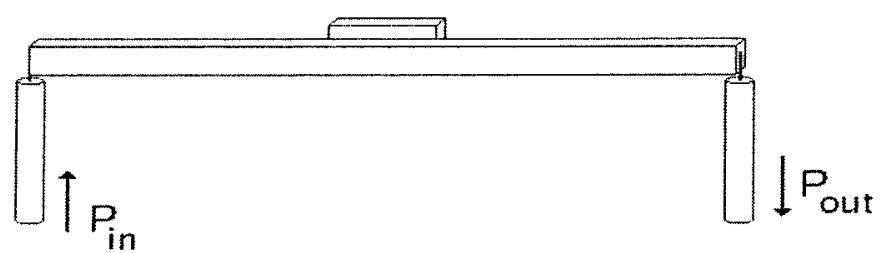

Figure 4.12: Power budget used to calculate total losses (ground plane omitted) 
The simulation setup used to isolate the radiation and return losses is depicted in Figure 4.13. Perfectly matched layers (PML) were used to absorb most of the energy in the DIG. The PML's allowed the second port to be omitted, which otherwise would have resulted in additional radiation and return losses. The materials in the simulation had no losses. The variables in Figure 4.13 correspond to the proceeding list of definitions.

$\boldsymbol{P}_{\text {in }}$ Input power

$\boldsymbol{P}_{\text {refl }}^{\boldsymbol{T R} \boldsymbol{R} \boldsymbol{N}}$ Reflected power due to the input transition

$\boldsymbol{P}_{\text {rad }}^{\boldsymbol{T} \boldsymbol{R} N \boldsymbol{S}}$ Radiated power due to the input transition

$\boldsymbol{P}_{\text {refl }}^{D \boldsymbol{R A}}$ Reflected power in the DIG due to the DRA

$\mathbf{P}_{\text {co-pol }}^{\text {DRA }}$ Co-polarized radiated power due to the DRA

$\mathbf{P}_{\mathbf{x}-\text { pol }}^{\text {DRA }}$ Cross-polarized radiated power due to the DRA

$\mathbf{P}_{\text {DIG }}$ Forward propagating power in the DIG directly after the input transition

\section{Losses due to the DIG input transition}

The loss due to the transition was decomposed into radiated and reflected loss. Both types were determined in section 3.4 where the DIG transitions were characterized. The relevant parameters for four different DIG's, each with an optimized transition are given in Table 3.2. 


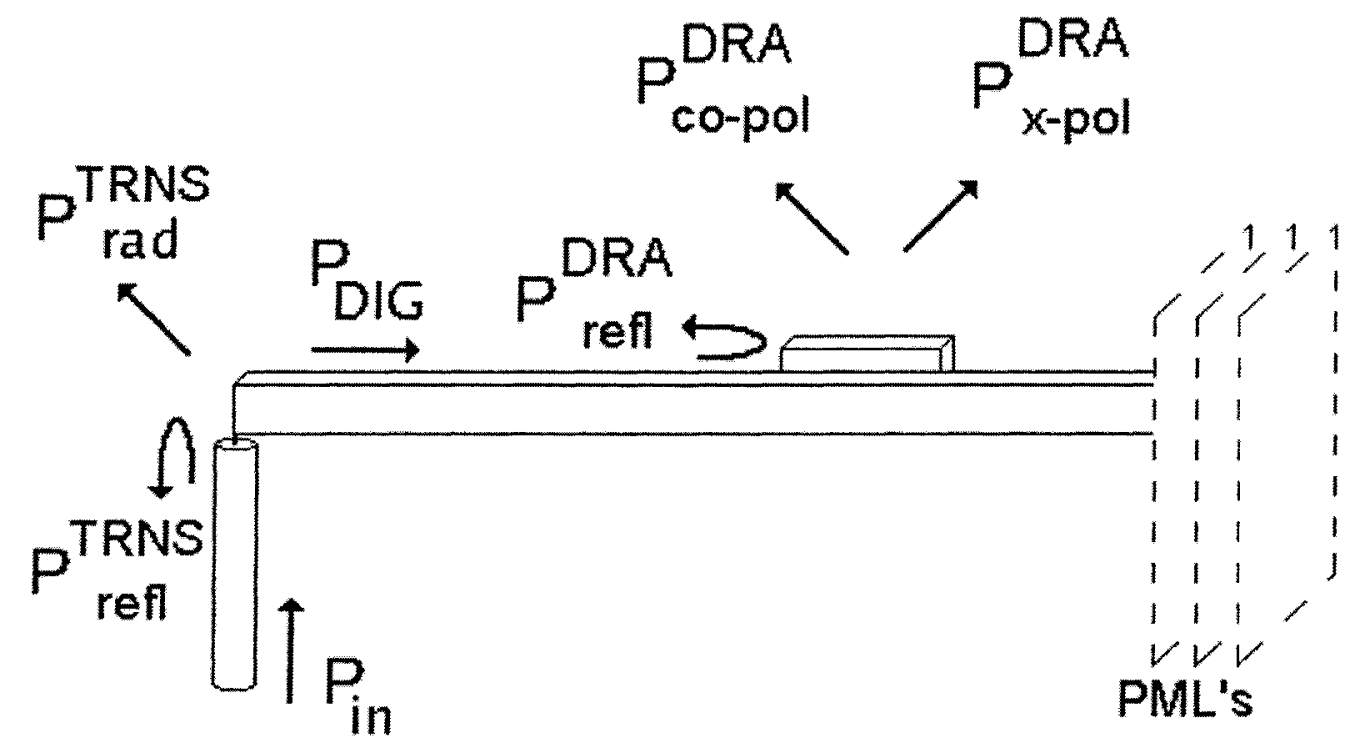

Figure 4.13: Power budget used to isolate losses in simulation results

\section{Return loss at DRA}

The presence of the DRA presented a discontinuity to the energy propagating in the bound mode of the DIG, therefore some of the energy reflected back towards the input port. The magnitude of the reflected wave increased as the spacing between the DRA and DIG decreased. To isolate this reflection, the time domain responses from the simulator were used to gate out all other reflections for the setup shown in Figure 4.13. Figure 4.14(a) shows the total reflected response in the time domain without a DRA present. This reflection is due solely to the input transition. Figure 4.14(b) shows the reflected response with a DRA present. The difference between these two time domain responses was used to isolate the reflection due solely to the DRA, which is shown in Figure 4.14(c). The discrete Fourier transform was applied to this time domain 
response to produce the frequency domain response in Figure 4.14(d). This figure is the $s_{11}$ due to the DRA. This reflection coefficient was calculated at the input port, but was required at the DRA location. In other words the ratio $\frac{P_{r e f l-a t-i n p u t}^{D R A}}{P_{\text {in }}}$ was calculated, but $\frac{P_{r e f l}^{D R A}}{P_{D I G}}$ was required. The term $P_{r e f l-a t-i n p u t}^{D R A}$ is measured at the input port, and is the remnants of $P_{\text {refl }}^{D R A}$ after it passes through the input transition. The relationships required to transform the reflection coefficient from the input to the DIG are:

$$
\begin{aligned}
& P_{D I G}=T^{2} \times P_{i n} \\
& P_{\text {refl-at-input }}^{D R A}=T^{2} \times P_{\text {refl }}^{D R A}
\end{aligned}
$$

The parameter $T$ is the transmission coefficient from the input coaxial cable to the DIG. It's definition is given by (4.5). All of the terms in (4.4) - (4.6) are on a linear scale.

$$
T^{2}=\left(1-\frac{P_{r a d}^{T R N S}+P_{r e f l}^{T R N S}}{P_{i n}}\right)
$$

The return loss at the DRA location is related to the return loss at the coaxial cable input by the relationships in (4.4).

$$
\frac{P_{r e f l}^{D R A}}{P_{D I G}}=\frac{1}{T^{4}} \frac{P_{r e f l-a t-i n p u t}^{D R A}}{P_{i n}}
$$

or in the logarithmic scale:

$$
\left.\frac{P_{r e f l}^{D R A}}{P_{D I G}}\right|_{d B}=\left.\frac{P_{r e f l-a t-i n p u t}^{D R A}}{P_{i n}}\right|_{d B}-10 \log _{10} T^{4}
$$


The results for several DRA's are shown in Table 4.1. The case number in the first column corresponds to the DIG parameters in Table 3.1. The last column in the Table shows the return loss due to the DRA is very small, and therefore can be neglected.

Table 4.1: Reflection due to presence of DRA at $30 \mathrm{GHz}$

\begin{tabular}{|c|c|c|c|}
\hline Case & $\mathrm{s}(\mathrm{mm})$ & $\left|s_{11}\right|$ due to $P_{\text {refl }}^{D R A}(\mathrm{~dB})$ & $\frac{P_{\boldsymbol{r} \text { efl }}^{\text {DA }}}{P_{\text {DIG }}}(\mathrm{dB})$ \\
\hline \hline$i$ & 2.25 & -18.0 & -0.032 \\
\hline$i$ & 2.5 & -25.8 & -0.005 \\
\hline$i$ & 2.75 & -34.4 & -0.001 \\
\hline$i v$ & 2.0 & -16.5 & -0.138 \\
\hline$i v$ & 2.25 & -23.5 & -0.027 \\
\hline$i v$ & 2.5 & -28.6 & -0.008 \\
\hline
\end{tabular}




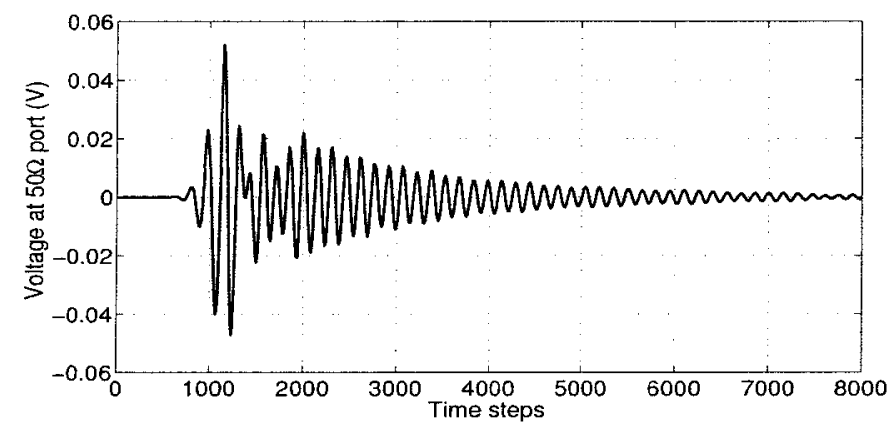

(a) Reflection time domain voltage at port 1 without DRA

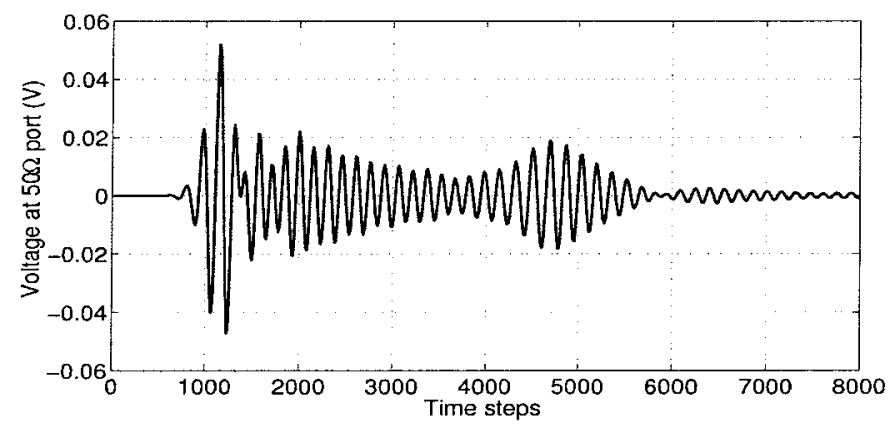

(b) Reflection time domain voltage at port 1 with DRA

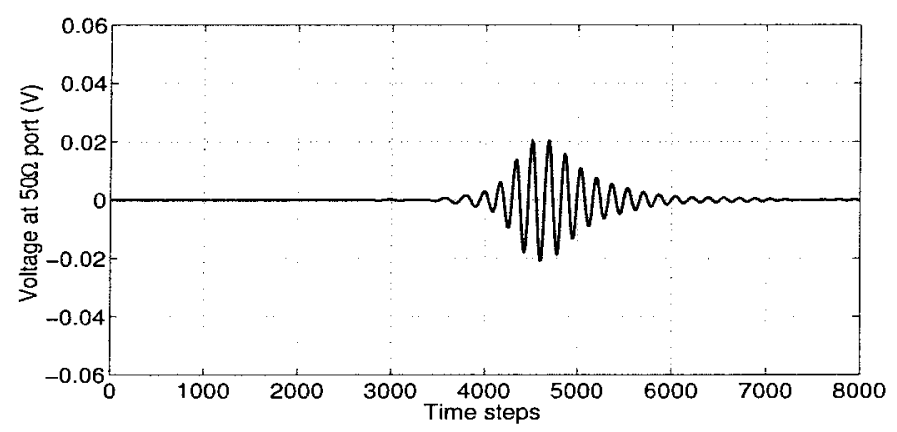

(c) Modified reflection time domain voltage at port 1

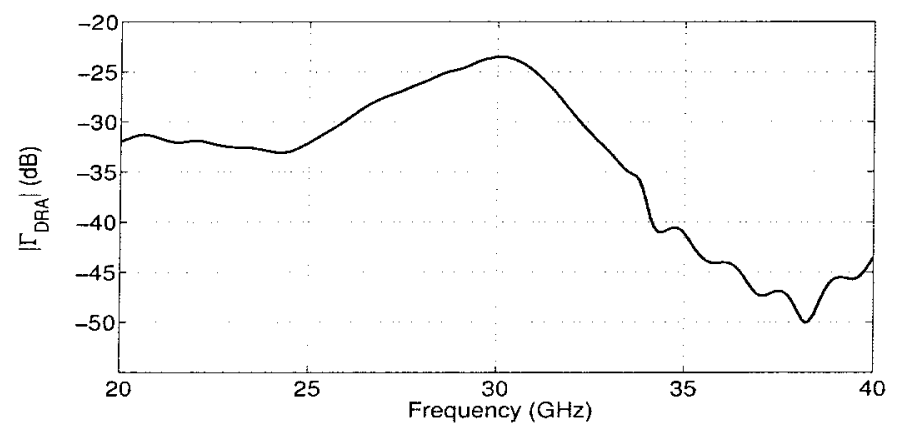

(d) Reflection at port 1 due solely to presence of DRA

Figure 4.14: Manipulation of time domain data to isolate DRA reflection 


\section{Radiation losses at DRA}

The radiation due to the presence of the DRA is due to the excitation of the desired $T E_{\delta 11}^{x}$ mode, the excitation of undesired radiating modes, and spurious radiation due to the discontinuity that the DRA presents. The co-polarized (co-pol) radiation was assumed to be solely due to the desired radiating mode of the DRA. The cross-polarized (x-pol) radiation was made up of other contributions.

The total radiation loss from the DRA was calculated by applying conservation of energy to the setup in Figure 4.12. The power budget for this problem was used to calculate the radiation losses from the DRA, and is given by (4.8) in the logarithmic scale.

$$
\begin{aligned}
s_{21} & =\sum \operatorname{losses}_{d B} \\
& =2 \times\left.\frac{P_{r a d}^{T R N S}+P_{r e f l}^{T R N S}}{P_{i n}}\right|_{d B}+\left.\frac{P_{x-p o l}^{D R A}+P_{c o-p o l}^{D R A}}{P_{D I G}}\right|_{d B} \\
& \left.\therefore \frac{P_{x-p o l}^{D R A}+P_{c o-p o l}^{D R A}}{P_{D I G}}\right|_{d B}=s_{21}-2 \times\left.\frac{P_{r a d}^{T R N S}+P_{r e f l}^{T R N S}}{P_{i n}}\right|_{d B}
\end{aligned}
$$

The simulation results from the setup in Figure 4.13 were used to decompose the total radiation loss from the DRA into co-pol and x-pol components. The first step in determining the co-pol radiation due solely to the DRA was subtracting out the radiation due to the input transition $\left(E^{\text {no } D R A}\right)$ from the total 
radiation pattern $\left(E^{t o t}\right)$.

$$
\begin{aligned}
& E_{c o-p o l}=E_{c o-p o l}^{t o t}-E_{c o-p o l}^{n o D A} \\
& E_{x-p o l}=E_{x-p o l}^{t o t}-E_{x-p o l}^{n o D R A}
\end{aligned}
$$

Empire was used to calculate the far-field at 5 degree increments for both $\theta$ and $\phi$ over an entire unit sphere. The ratio of co-pol radiated power to the total radiated power was calculated by numerically integrating the 3-D far-field patterns for both the co-pol radiation and the total radiation. One case was repeated using a 3 degree increment for both angles and the results differed by a small amount, thereby justifying the coarser 5 degree step. The equations used to numerically integrate the fields are shown in (4.10)-(4.12).

The average radiated power is given by [8]:

$$
\begin{aligned}
P_{a v e} & =\frac{1}{2 \eta_{o}} \iint_{\Omega}\left(E_{c o-p o l}^{2}+E_{x-p o l}^{2}\right) d \Omega \\
& =\frac{1}{2 \eta_{o}} \int_{0}^{2 \pi} \int_{0}^{\pi}\left(E_{c o-p o l}^{2}+E_{x-p o l}^{2}\right) r^{2} \sin \theta d \theta d \phi \\
& \approx \frac{r^{2}}{2 \eta_{o}} \sum_{n=1}^{N} \sum_{m=1}^{M}\left[E_{c o-p o l}^{2}\left(\theta_{n}, \phi_{m}\right)+E_{x-p o l}^{2}\left(\theta_{n}, \phi_{m}\right)\right] \sin \theta_{n} \Delta \phi \Delta \theta
\end{aligned}
$$

Similarly the co-pol radiated power is:

$$
P_{\text {co-pol }} \approx \frac{r^{2}}{2 \eta_{o}} \sum_{n=1}^{N} \sum_{m=1}^{M} E_{c o-p o l}^{2}\left(\theta_{n}, \phi_{m}\right) \sin \theta_{n} \Delta \phi \Delta \theta
$$


The ratio of co-pol radiated power to the total radiated power was obtained by dividing the result from equation 4.11 by that of 4.10 . This ratio is shown below.

$$
\frac{P_{\text {co-pol }}}{P_{\text {total }}} \approx \frac{\sum_{n=1}^{N} \sum_{m=1}^{M} E_{c o-p o l}^{2}\left(\theta_{n}, \phi_{m}\right)}{\sum_{n=1}^{N} \sum_{m=1}^{M}\left[E_{c o-p o l}^{2}\left(\theta_{n}, \phi_{m}\right)+E_{x-p o l}^{2}\left(\theta_{n}, \phi_{m}\right)\right]}
$$

\subsubsection{Results}

The ratio of co-pol power radiated by the DRA $\left(P_{c o-p o l}^{D R A}\right)$ to the power in the DIG $\left(P_{D I G}\right)$ was considered to be a satisfactory approximation for the amount of power that is coupled from the DIG into the $T E_{\delta 11}^{x}$ mode of the DRA. The losses for several different DIG's with resonant DRA's located at different distances from them are summarized in Table 4.2 .

The last two columns in Table 4.2 report the analytical and simulation results for the power coupling into a single DRA. The graphs in Figure 4.15 compare the simulation results to the analytical approximation. The common factor among the results is that the analytical approximation appears to break down when the DIG-DRA spacing is very small. This result is expected since CMT for DIG couplers also breaks down when the guides are very close. The analytical and simulation results converge for DIG's with small cross-section dimensions and permittivity. A parameter that accounts for the dimensions and permittivity is the phase velocity $\left(v_{p}\right)$ of the DIG. The value of $v_{p} / c$ is shown for different DIG's in Figure 4.15, with the larger values corresponding 
Table 4.2: Summary of losses for a single DRA for various cases

\begin{tabular}{|c|c|c|c|c|c|c|}
\hline Case & $\begin{array}{c}\mathrm{s} \\
(\mathrm{mm})\end{array}$ & $\begin{array}{l}L_{D R A} \\
(\mathrm{~mm})\end{array}$ & $\begin{array}{c}s_{21} \\
(\mathrm{~dB})\end{array}$ & $\frac{P_{\text {co-pol }}^{D R A}}{P_{\text {total }}^{D R A}}(\%)$ & $\begin{array}{l}\text { Simulated } \\
\frac{P_{c o-p o l}^{D R A}}{P_{D I G}}(\%)\end{array}$ & $\begin{array}{l}\text { Analytical } \\
\frac{P_{c o-p o l}^{D R A}}{P_{D I G}}(\%)\end{array}$ \\
\hline $\bar{i}$ & 2.25 & 4.75 & -6.0 & $\overline{884.1}$ & $\overline{440.5}$ & 64.70 \\
\hline$i$ & 2.5 & 4.5 & -5.0 & 85.9 & 29.8 & 41.18 \\
\hline$i$ & 2.75 & 4.25 & -4.1 & 87.1 & 17.1 & 23.97 \\
\hline$i$ & 3.0 & 4.0 & -3.8 & 87.9 & 12.2 & 13.46 \\
\hline$i$ & 3.25 & 3.75 & -3.53 & 89.0 & 7.45 & 7.45 \\
\hline$i$ & 3.5 & 3.75 & -3.31 & 88.6 & 3.2 & 4.70 \\
\hline$i i$ & 2.5 & 3.0 & -4.1 & 74.4 & 22.9 & 14.10 \\
\hline$i i$ & 2.75 & 2.75 & -3.3 & 74.9 & 12.6 & 6.61 \\
\hline$i i$ & 3.0 & 2.75 & -2.86 & 80.1 & 6.4 & 3.62 \\
\hline$i i i$ & 2.5 & 4.75 & -5.40 & 74.9 & 23.7 & 35.2 \\
\hline$i i i$ & 2.75 & 4.75 & -4.97 & 77.5 & 19.0 & 28.6 \\
\hline$i i i$ & 3.0 & 4.5 & -4.64 & 78.0 & 14.5 & 19.0 \\
\hline$i i i$ & 3.25 & 4.5 & -4.53 & 80.9 & 13.3 & 13.6 \\
\hline$i i i$ & 3.5 & 4.25 & -4.21 & 81.8 & 8.2 & 8.7 \\
\hline$i v$ & 2.0 & 3.0 & -2.7 & 85.6 & 30.8 & 17.69 \\
\hline$i v$ & 2.25 & 2.75 & -1.4 & 84.1 & 11.5 & 8.12 \\
\hline$i v$ & 2.5 & 2.75 & -1.19 & 86.2 & 8.1 & 4.41 \\
\hline
\end{tabular}

to the smaller cross-sections and lower permittivity. When two dimensions of a DRA are decreased, then the third must increase to maintain resonance. When these two dimensions are in the cross-section, then this third dimension is parallel to the DIG. As this dimension increases the DRA becomes more slender and better represents a long DIG. The analytical approximation assumes the DRA is a DIG, therefore this result was expected. 


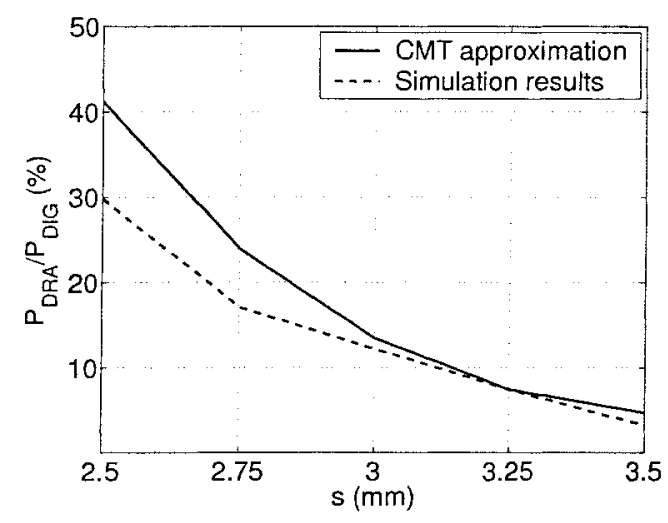

(a) Case $i: v_{p} / c=0.65$

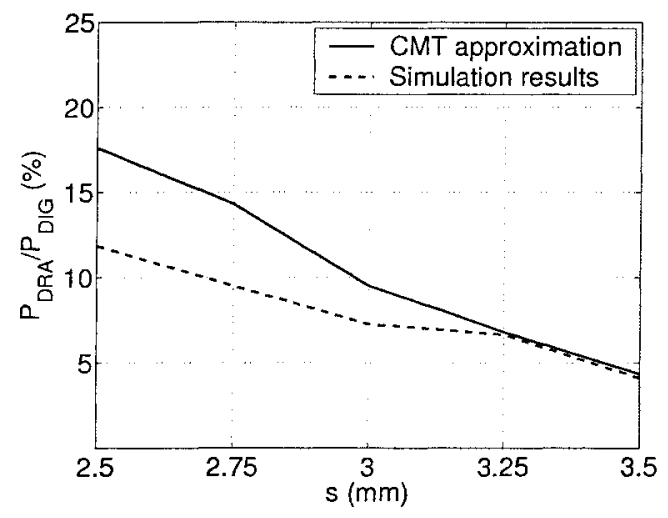

(c) Case $i i i: v_{p} / c=0.76-\mathrm{fix}$

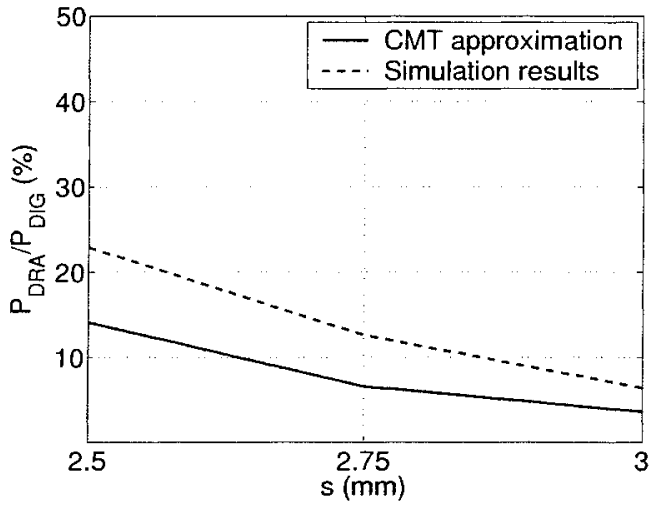

(b) Case $i i: v_{p} / c=0.52$

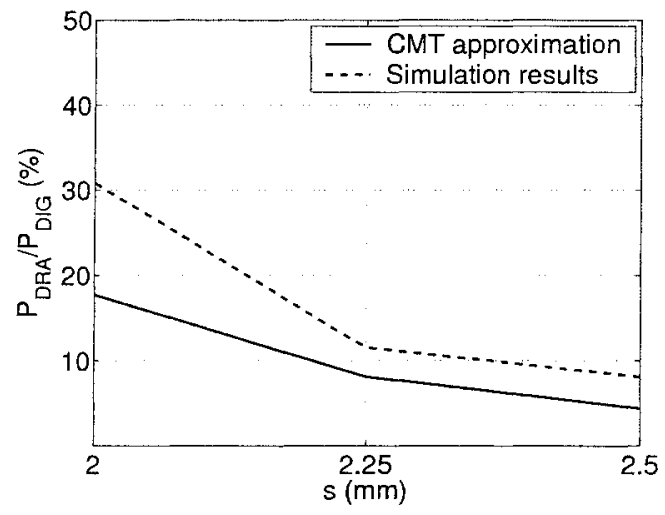

(d) Case $i v: v_{p} / c=0.51$

Figure 4.15: Comparison of simulation results to analytical approximation

\subsection{Specific Amplitude Distributions}

Specific amplitude distributions are applied to antenna arrays primarily for sidelobe control or maximizing the gain. Several common amplitude distributions are listed below along with some of their characteristics.

Uniform: The uniform amplitude distribution provides the maximum gain. For arrays with more than 5-6 elements, the highest sidelobes are ap- 
proximately $13 \mathrm{~dB}$ below the main beam. All elements are excited with equal weights.

Binomial: The amplitude weights of the binomial array are the binomial coefficients. There is a very large variation between the minimum and maximum coefficients for a large binomial array, therefore it is difficult to realize in practice. The array factor has no sidelobes, but has a relatively wide main lobe.

Dolph-Chebyshev: For a maximum specified sidelobe level, the minimum beam width will be achieved when all of the sidelobes are equal to that maximum level [8]. This principle is adopted by the Chebyshev array to minimize the main lobe while controlling the sidelobe level.

Taylor: The maximum sidelobe level for a specific number of lobes nearest to the main lobe is a design parameter for a Taylor distribution. The roll-off of the sidelobe levels for the lobes further away decays as $\sin (x) / x$. The Villeneuve distribution implements the Taylor distribution for a discrete array [48].

A non-resonant transmission line was considered. This type of line has an output port that absorbs most of the energy incident upon it. Figure 4.16 shows an example of this type of array with three radiating elements. The DIG has been divided into three unit cells, each corresponding to a DRA. The length of each unit cell is equivalent to the element spacing of the array $(d)$. Each unit cell couples a fraction of the power from the DIG into the DRA $\left(p_{n}\right)$ 


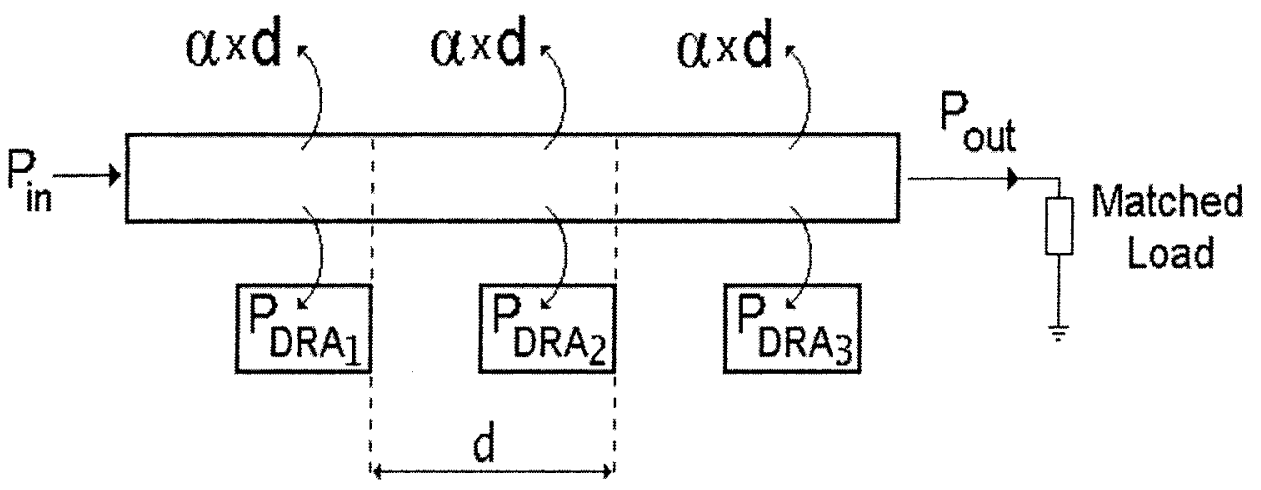

Figure 4.16: Array fed by non-resonant DIG

and dissipates some energy due to material losses $(\alpha \times d)$. These variables are defined mathematically in (4.13).

$$
\begin{aligned}
& p_{n}=\frac{P_{D R A_{n}}}{P_{D I G_{n}}} \\
& \alpha=\alpha_{c}+\alpha_{d} \quad(\mathrm{~dB} / \mathrm{cm})
\end{aligned}
$$

For maximum efficiency $100 \%$ of the input power must be radiated, but this would require the last array element to extract all of the remaining power from the DIG. This requirement was found to be impractical, even with lossless materials. The fraction of power that is left in the guide is given by $\frac{P_{o u t}}{P_{i n}}$. The required power distribution is calculated using the required amplitude coefficients $\left(A_{n}\right)$, as shown in (4.14). 


$$
\frac{P_{D R A_{n}}}{P_{\text {in }}}=\left(1-\frac{P_{\text {out }}}{P_{\text {in }}}\right) \times \frac{A_{n}^{2}}{\sum_{m=1}^{N} A_{m}^{2}}
$$

After an amplitude distribution is specified, the values of $p_{n}$ must be determined for each DRA. These values are used to locate each DRA at a specific distance away from the DIG. The following equations show the derivation of the relationship between $p_{n}$ and the other array parameters.

$$
\begin{aligned}
& P_{D R A_{1}}=P_{i n}\left(10^{-\alpha d / 10}\right) p_{1} \\
& P_{D R A_{2}}=P_{i n}\left(10^{-\alpha d / 10}\right)^{2}\left(1-p_{1}\right) p_{2} \\
& \Rightarrow P_{D R A_{n}}=P_{i n}\left(10^{-\alpha d / 10}\right)^{n} p_{n} \prod_{m=1}^{n-1}\left(1-p_{m}\right) \\
& \therefore p_{1}=\frac{P_{D R A_{1}}}{P_{i n}} \times 10^{\alpha d / 10} \\
& \& \quad p_{n}=\frac{P_{D R A_{n}}}{P_{i n}} \times 10^{n \alpha d / 10} \times \prod_{m=1}^{n-1}\left(1-p_{m}\right)^{-1} \text { for } n=2,3 \ldots N
\end{aligned}
$$

Equations (4.14) and (4.15) are used to determine the required amount of power to couple into each element. Using the design data for the equivalent directional coupler obtained by CMT and the DIG loss, the length and spacing of each DRA can be determined for a specified amplitude distribution. 


\subsubsection{DRA Array with Uniform Amplitude Distribution}

An 8-element array was designed with a uniform amplitude distribution. The array was to radiate $75 \%$ of the input power, which was based on the expected material losses. These specifications are represented mathematically as:

$$
\begin{aligned}
& A_{n}=1 \quad \text { for all } n \\
& \frac{P_{\text {out }}}{P_{\text {in }}}=0.25
\end{aligned}
$$

The dimensions of the DIG were chosen to correspond to case $i$ of Table 3.1. The losses of this guide were reported in Table 3.1. The array specifications were entered into (4.14) and (4.15) along with the loss properties of the DIG. The array's element spacing was chosen to be the minimum value where mutual coupling effects were not significant. Mutual coupling effects were determined by simulation. An element spacing of $d=0.8 \mathrm{~mm}$ was chosen. Table 4.3 displays the parameters $A_{n}$ and $p_{n}$ for all the elements of the array. To realize the required $p_{n}$ values, the length and separation distance for each DRA was chosen from the coupling curve of Figure 4.8. The DRA length and spacing were discretized into $0.25 \mathrm{~mm}$ increments to make fabrication simpler. This discretization also discretizes the actual $p_{n}$ values, therefore they will not ex-

actly match the required values, hence the predicted $p_{n}$ does not equal the required $p_{n}$ in Table 4.3. 
Table 4.3: 8-element array with uniform amplitude distribution

\begin{tabular}{|c|c|c|c|c|c|}
\hline $\mathrm{n}$ & $A_{n}$ & $\begin{array}{c}\text { Required } \\
p_{n} \%\end{array}$ & $s_{n}(\mathrm{~mm})$ & $L_{D R A_{n}}(\mathrm{~mm})$ & $\begin{array}{c}\text { Predicted } \\
p_{n} \%\end{array}$ \\
\hline \hline 1 & 1.0 & 9.8 & 3.25 & 3.5 & 6.51 \\
\hline 2 & 1.0 & 11.3 & 3.0 & 3.75 & 11.9 \\
\hline 3 & 1.0 & 13.2 & 3.0 & 3.75 & 11.9 \\
\hline 4 & 1.0 & 15.9 & 2.75 & 3.75 & 19 \\
\hline 5 & 1.0 & 19.6 & 2.75 & 3.75 & 19 \\
\hline 6 & 1.0 & 25.4 & 2.75 & 4.0 & 21.45 \\
\hline 7 & 1.0 & 35.5 & 2.5 & 4.0 & 33.69 \\
\hline 8 & 1.0 & 57.4 & 2.25 & 4.5 & 59.93 \\
\hline
\end{tabular}

\section{Expected versus Simulation}

The expected radiation pattern was obtained using ARPS, an array plotting software. The DRA's were modeled by small apertures in an infinite ground plane. The predicted values of $p_{n}$ from Table 4.3 were used to determine the predicted $A_{n}$ values. The patterns are shown in Figure 4.17. In this figure a grating lobe is visible, which is due to the element spacing. The grating lobe was deemed acceptable since the array was designed to validate the analytical analysis method. This method does not account for mutual coupling effects, therefore it was important that these effects were negligible. Empire was used to simulate the array. The simulated radiation patterns are shown in Figure 4.18. Figure 4.18 shows the radiation pattern with the feed radiation subtracted. The feed radiation was subtracted so the radiation patterns could be fairly compared to those in Figure 4.17. To analyze the bandwidth, the simulated radiation pattern is reported in Figure 4.19 for several frequencies. 


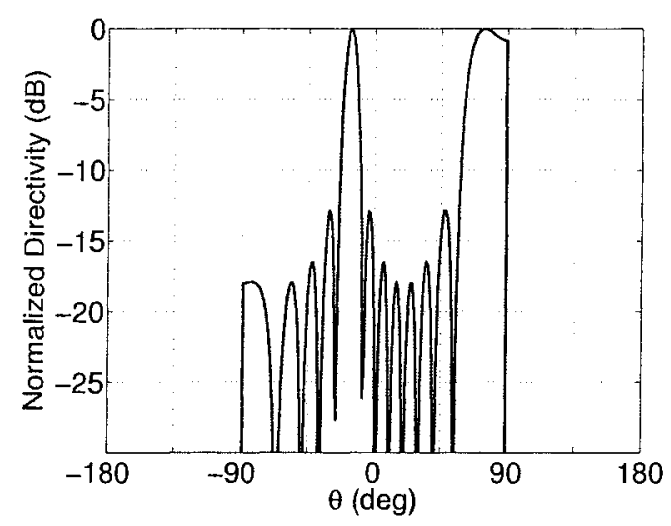

(a) E-plane

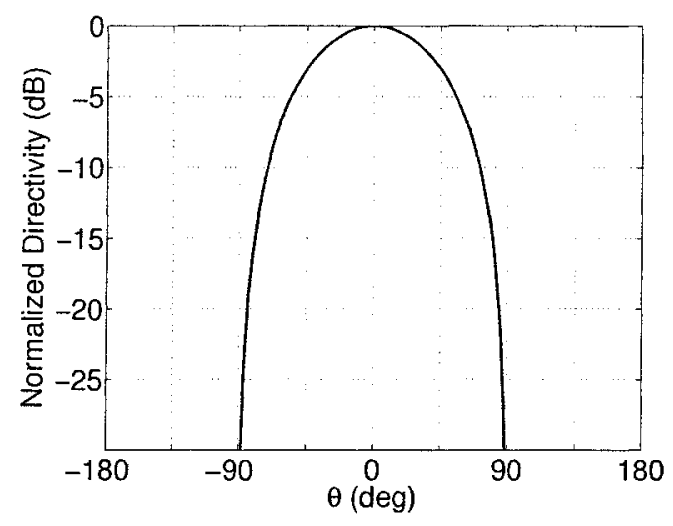

(b) H-plane

Figure 4.17: 8-element array with uniform amplitude distribution - ARPS

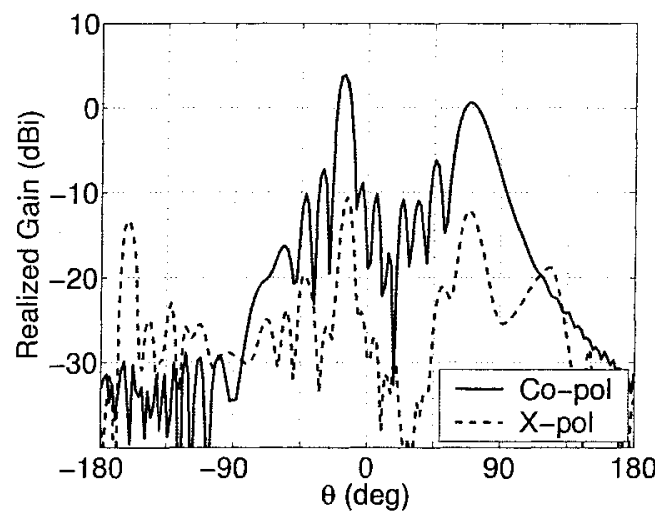

(a) E-plane

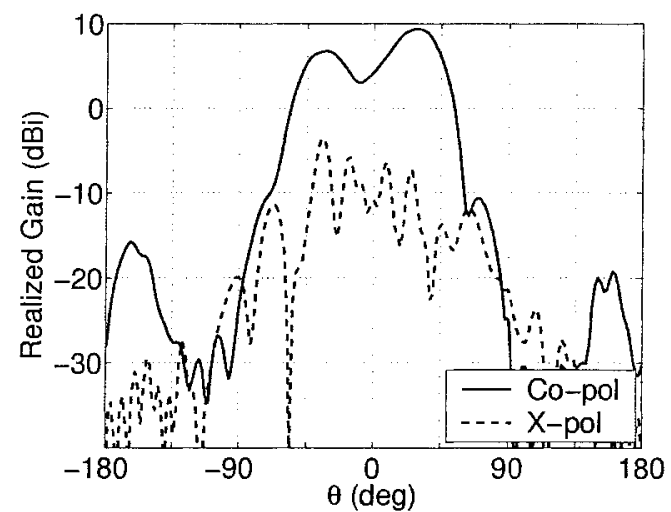

(b) H-plane

Figure 4.18: 8-element array with uniform amplitude distribution - Empire

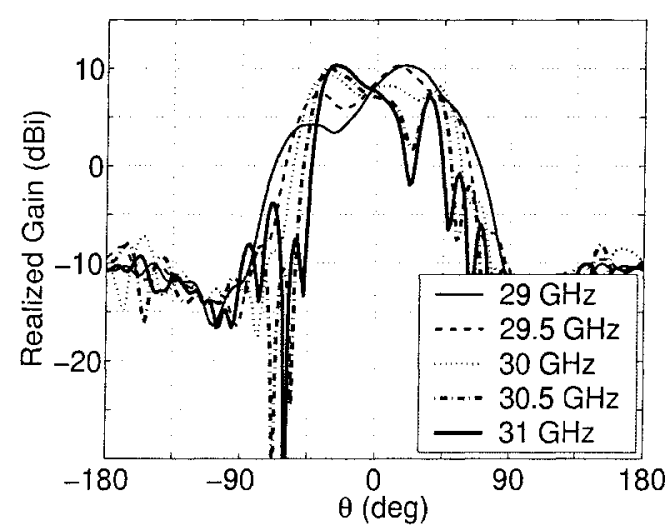

Figure 4.19: Simulated gain with amplitude distribution in the H-plane 
The maximum sidelobe level of the array was expected to be $13 \mathrm{~dB}$ below the main beam, and the simulation result in Figure 4.32(a) shows a sidelobe level of $-11 \mathrm{~dB}$ on one side of the main beam and $-13 \mathrm{~dB}$ on the other. Good agreement exists between the expected and simulated location of the main beam and grating lobe. This result indicates that the correct phase distribution was achieved across the array. In the H-plane there is a large ripple in the co-polarized curve. This effect is due to the presence of the DIG and decreases the gain in the $\theta=0^{\circ}$ direction. This issue is dealt with in section 5.1.

\subsubsection{DRA Array with Chebyshev Amplitude Distribution}

The characteristic feature of the Dolph-Chebyshev array factor is equal amplitude sidelobes. The amplitude coefficients were determined using ARPS. An 8-element array was designed which was to have sidelobes at $30 \mathrm{~dB}$ below the peak. The required amplitude coefficients for the array elements are shown in Table 4.4. The fraction of the input power that was radiated was designed to be $75 \%$.

$$
\frac{P_{\text {out }}}{P_{\text {in }}}=0.25
$$

The same DIG dimensions and permittivity were used as with the uniform amplitude distribution in the preceding section. 
Table 4.4: 8-element array with Chebyshev amplitude distribution

\begin{tabular}{|c|c|c|c|c|c|}
\hline $\mathrm{n}$ & $A_{n}$ & $\begin{array}{c}\text { Required } \\
p_{n} \%\end{array}$ & $s_{n}(\mathrm{~mm})$ & $L_{D R A_{n}}(\mathrm{~mm})$ & $\begin{array}{c}\text { Predicted } \\
p_{n} \%\end{array}$ \\
\hline \hline 1 & 0.579 & 5.16 & 3.5 & 3.5 & 4.0 \\
\hline 2 & 0.660 & 7.36 & 3.25 & 3.5 & 6.5 \\
\hline 3 & 0.875 & 14.55 & 3.0 & 3.75 & 12.0 \\
\hline 4 & 1.0 & 23.16 & 2.75 & 3.75 & 19.0 \\
\hline 5 & 1.0 & 31.39 & 2.5 & 4.0 & 34.0 \\
\hline 6 & 0.875 & 36.47 & 2.5 & 4.0 & 34.0 \\
\hline 7 & 0.660 & 34.01 & 2.5 & 4.0 & 34.0 \\
\hline 8 & 0.579 & 41.31 & 2.5 & 4.25 & 37.0 \\
\hline
\end{tabular}

\section{Ideal versus Simulation}

The ideal radiation pattern was obtained using ARPS. The patterns are shown in Figure 4.20. The DRA's were modeled by small apertures, and an infinite ground plane approximation applied. The loss due to the input transition and material losses must be accounted for when comparing the directivity of this result to the realized gain from simulations and measured radiation patterns. The simulated radiation patterns for the array are shown in Figure 4.21. In these plots the radiation due to the feed has been subtracted from the total radiation.

The radiation pattern in Figure 4.21(a) has a maximum sidelobe level of 16 $\mathrm{dB}$ below the main peak on one side of the main beam and $12 \mathrm{~dB}$ on the other. These sidelobe levels are lower than that for the array in the previous section with a uniform amplitude distribution, therefore some sidelobe control has been demonstrated. The intended level was $30 \mathrm{~dB}$ down, which is not close 


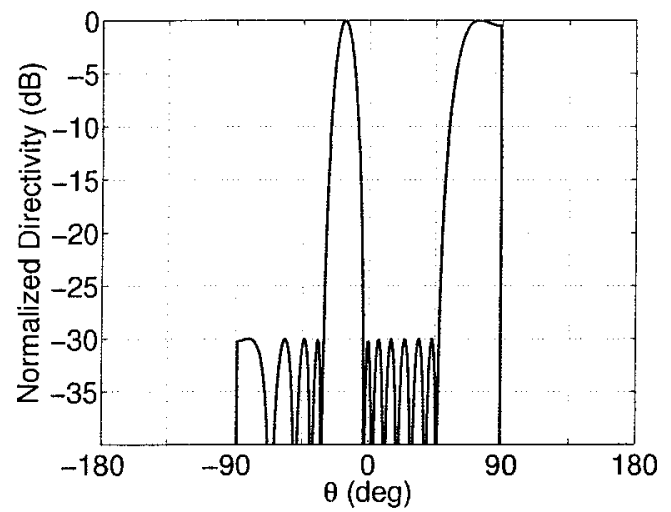

(a) E-plane

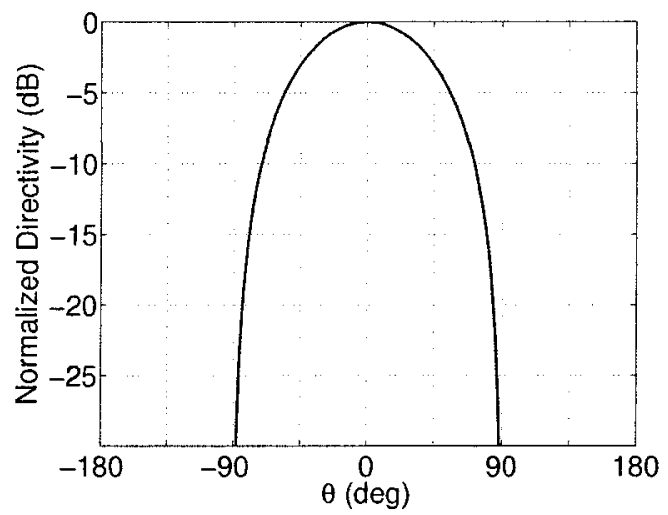

(b) H-plane

Figure 4.20: 8-element array with Chebyshev amplitude distribution - ARPS

to that achieved. The discretization of the DRA sizes and spacings attributed to this error. Furthermore, accurate amplitude excitation of the elements was not expected from the CMT approximation for the DRA's that were located closely to the DIG. The finite size ground plane caused interference to the radiation pattern, as well as some mutual coupling.

The bandwidth was investigated by comparing the radiation pattern in the H-plane at different frequencies. These patterns are shown in Figure 4.22. In this figure there is a relatively large variation in the radiation pattern as the frequency deviates from $30 \mathrm{GHz}$. It was found that as the main beam moves away from the $\theta=0^{\circ}$ direction the radiation pattern deteriorates from that of an ideal short magnetic dipole. This result is due to the interference with the DIG. 


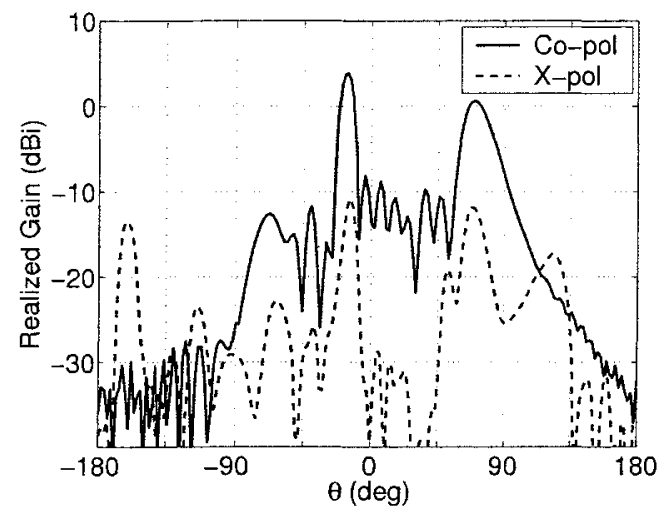

(a) E-plane

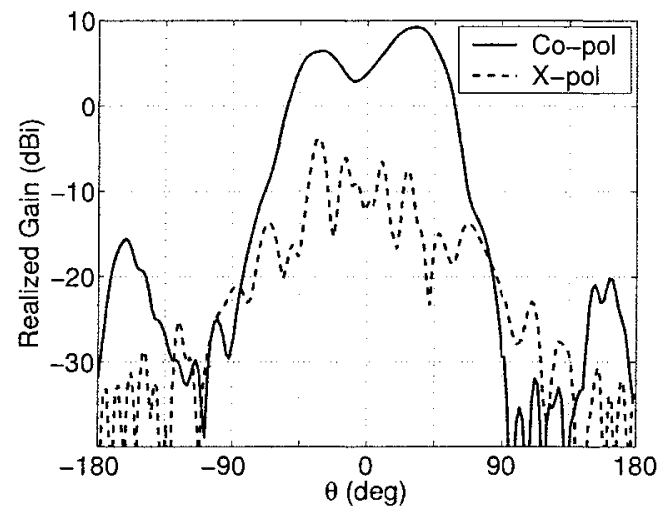

(b) H-plane

Figure 4.21: 8-element array with Chebyshev amplitude distribution - Empire

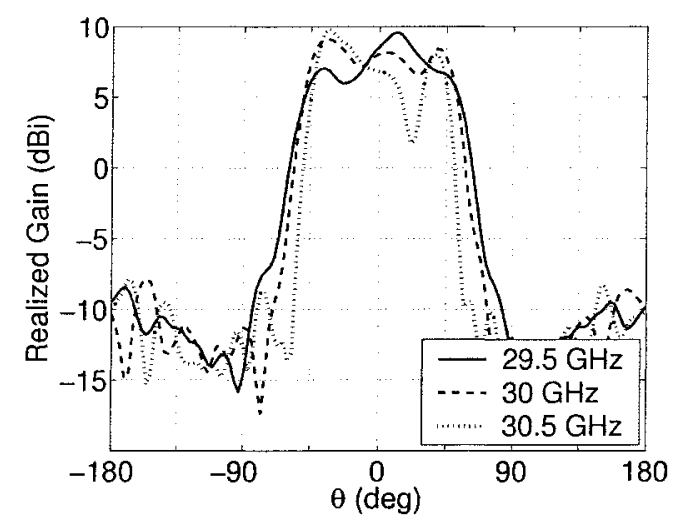

Figure 4.22: Simulated gain with Chebyshev distribution in the H-plane

\subsubsection{DRA Array with Taylor Amplitude Distribution}

The design guidelines were used to generate a 16-element array with a Taylor amplitude distribution at $60 \mathrm{GHz}$. The DIG dimensions and permittivity were chosen such that the DIG supported a loosely bound mode $\left(v_{p} / c \approx 0.8\right)$ and did not have a small aspect ratio $(h / w>1)$. A substrate with $\epsilon_{r}=3$ was chosen to ensure the DRA's had a relatively low quality factor. The dimensions 
of the guide were $h=1.905 \mathrm{~mm}$, and $w=1 \mathrm{~mm}$. The material properties were calculated based on those of Roger's RT/duroid $6002\left(\epsilon_{r}=2.94 \pm 0.040\right.$, $\tan \delta=0.0012)$. The guided wavelength and loss value of this guide are:

$$
\begin{aligned}
& \lambda_{g}=4.07 \mathrm{~mm} \\
& \alpha=0.08 \mathrm{~dB} / \mathrm{cm}
\end{aligned}
$$

The Taylor distribution for continuous apertures produces the Taylor pattern. This pattern can be produced by discrete arrays by applying the Villeneuve distribution [48]. For this distribution the number of equal amplitude sidelobes adjacent to the main beam are specified. Furthermore, the level of these sidelobes with respect to the main beam is specified. The specifications for the array designed in this section were:

i. the first 3 sidelobes adjacent to the main beam are to be $25 \mathrm{~dB}$ down from the main lobe

ii. $80 \%$ of the input power is to be radiated $\left(P_{\text {out }} / P_{\text {in }}=0.8\right)$

The power coupling properties of a DRA in proximity to this DIG was predicted using CMT, and the results are shown in Figure 4.23(a). The length of the DRA was made to correspond to $1 / 2 \lambda_{11}$, which is a function of $s$. The required amplitude distribution and required coupling values for the array are shown in the second and third columns of Table 4.5. The corresponding values of $L_{D R A_{n}}$ and $s_{n}$ were chosen from Figure 4.23(b) to meet the array requirements. 


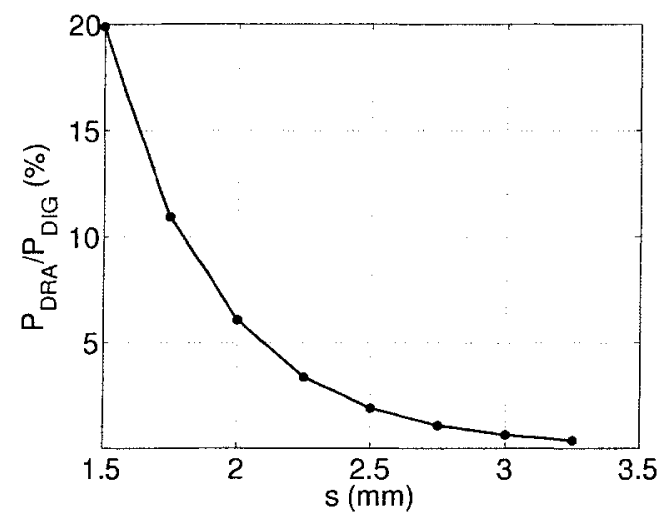

(a) Power coupling

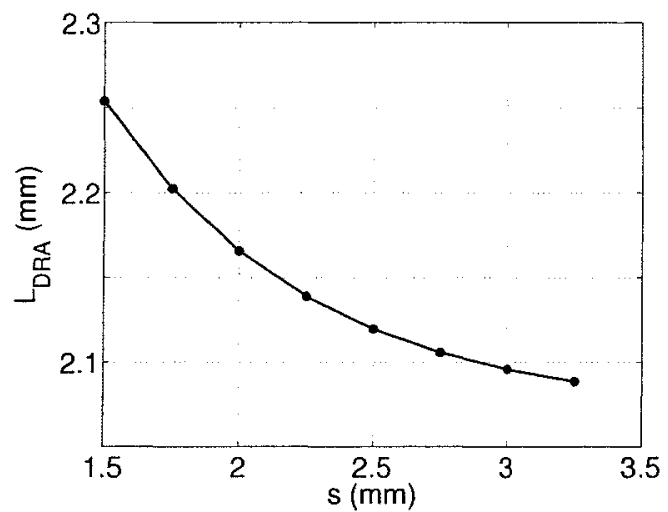

(b) DRA length

Figure 4.23: CMT results for DIG at $60 \mathrm{GHz}$

The expected radiation patterns were obtained using ARPS, and are shown in Figures 4.24(a)-4.24(b). Empire was used to simulate the array to compare it with the expected pattern. The simulated radiation patterns are shown in Figures $4.24(\mathrm{c})-4.24(\mathrm{~d})$. In this figure the feed radiation has been subtracted from the total radiation pattern. 
Table 4.5: 16-element array with Taylor amplitude distribution

\begin{tabular}{|c|c|c|c|c||c|c|c|c|c|}
\hline $\mathrm{n}$ & $A_{n}$ & $\begin{array}{c}p_{n} \\
(\%)\end{array}$ & $\begin{array}{c}s_{n} \\
(\mathrm{~mm})\end{array}$ & $\begin{array}{c}L_{D R A_{n}} \\
(\mathrm{~mm})\end{array}$ & $\mathrm{n}$ & $A_{n}$ & $\begin{array}{c}p_{n} \\
(\%)\end{array}$ & $\begin{array}{c}s_{n} \\
(\mathrm{~mm})\end{array}$ & $\begin{array}{c}L_{D R A_{n}} \\
(\mathrm{~mm})\end{array}$ \\
\hline \hline 1 & 0.36 & 1.2 & 2.7 & 2.1 & 9 & 1.0 & 17.1 & 1.5 & 2.4 \\
\hline 2 & 0.42 & 1.7 & 2.6 & 2.1 & 10 & 0.96 & 19.2 & 1.5 & 2.4 \\
\hline 3 & 0.52 & 2.7 & 2.4 & 2.2 & 11 & 0.88 & 20.2 & 1.5 & 2.4 \\
\hline 4 & 0.65 & 4.2 & 2.2 & 2.2 & 12 & 0.77 & 19.5 & 1.5 & 2.4 \\
\hline 5 & 0.77 & 6.4 & 2.0 & 2.2 & 13 & 0.65 & 17.1 & 1.5 & 2.4 \\
\hline 6 & 0.88 & 8.9 & 1.8 & 2.3 & 14 & 0.52 & 13.5 & 1.7 & 2.3 \\
\hline 7 & 0.96 & 11.7 & 1.7 & 2.3 & 15 & 0.42 & 10.2 & 1.8 & 2.3 \\
\hline 8 & 1.0 & 14.5 & 1.6 & 2.4 & 16 & 0.36 & 8.6 & 1.9 & 2.3 \\
\hline
\end{tabular}

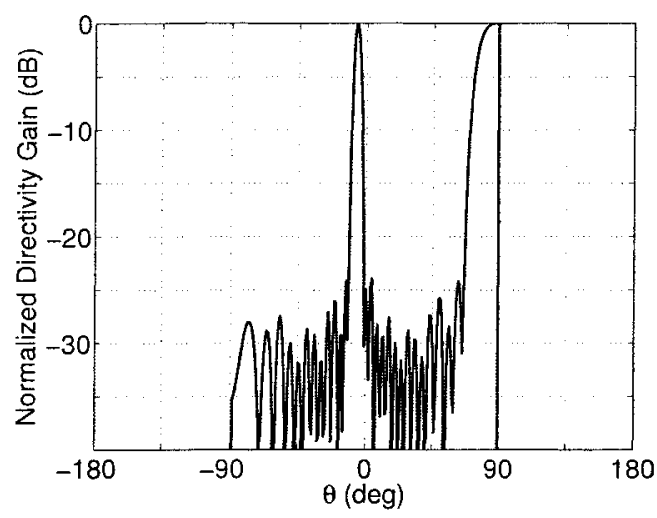

(a) ARPS: E-plane

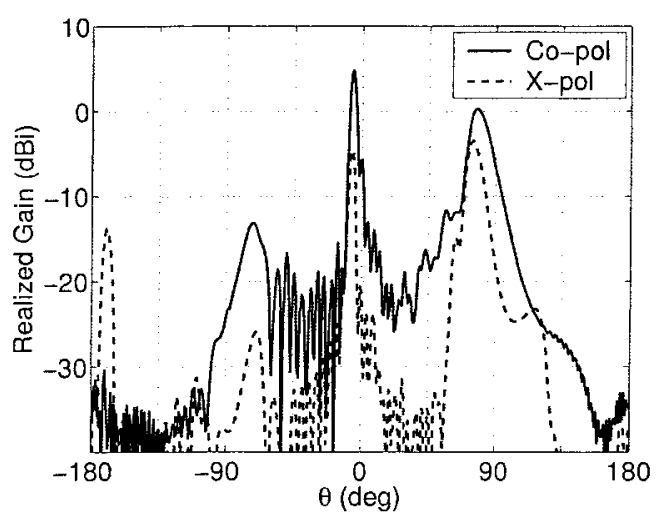

(c) Empire: E-plane

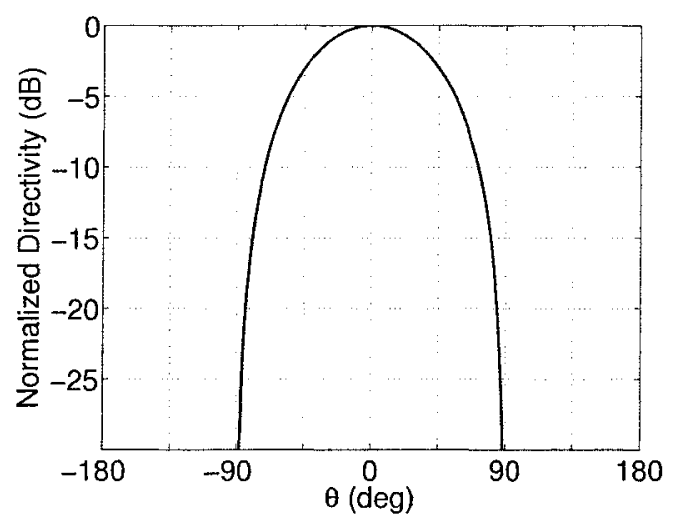

(b) ARPS: H-plane

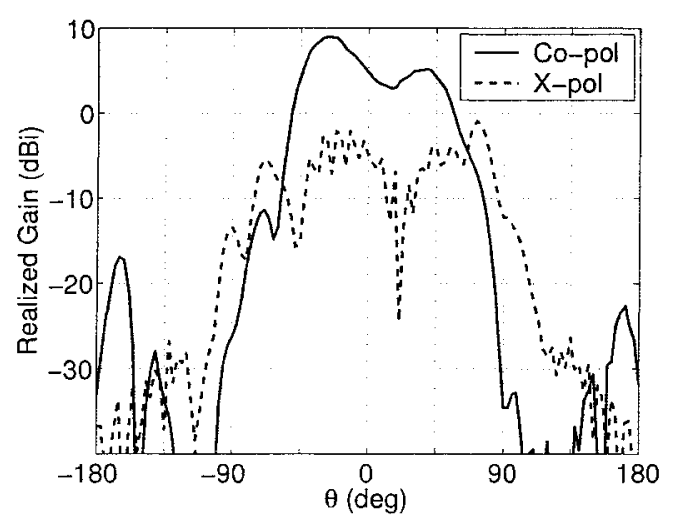

(d) Empire: H-plane

Figure 4.24: 16-element array with Taylor amplitude distribution 
The simulated radiation pattern in the array plane in Figure 4.24(c) shows sidelobe behaviour that is better than the case of an array with a uniform distribution. Specifically, the maximum sidelobe level on one side of the main beam is $20 \mathrm{~dB}$, but is only $10.5 \mathrm{~dB}$ below on the other side of the main beam. The adjacent sidelobes to this large one are $18 \mathrm{~dB}$ below the main beam. The location of the main beam from thee simulated results matches the expected result, but the location of the grating lobe shows some error. The direction of the grating lobe was expected to be parallel to the ground plane, but for the case of a finite ground plane it is common for the beam to tilt slightly up. This behaviour is consistent with the results. The H-plane pattern shows signs of the DIG interference that was common to the arrays designed in the preceeding sections.

\subsection{The DRA Array Fed by Microstrip}

The advantages of the DIG fed DRA array were investigated by comparing it's performance to a microstrip fed DRA array. An array of this type with a Taylor amplitude distribution was reported in the literature [49]. This 10element array was operated at $8.2 \mathrm{GHz}$, where a maximum gain of $13.2 \mathrm{dBi}$ was measured. The amplitude distribution was achieved by varying the spacing between the microstrip line and each DRA. An equivalent circuit consisting of a transmission line loaded with shunt conductances was used to analyze the array. The required shunt conductances to achieve the specified Taylor dis- 
tribution were known. DRA's were added to the array one-by-one with each being adjusted so that the required conductance of the entire line met the required value. This design process was done experimentally with a network analyzer. This array demonstrated the feasibility of microstrip line as a series feed for a DRA array at $8.2 \mathrm{GHz}$. At a higher frequency the energy would have been more tightly bound to the substrate, thereby deteriorating the coupling into the DRA's. Furthermore, as the frequency increases microstrip line dimensions become smaller and material losses increase. These challenges were tackled to design an array similar to that in [49], but at $30 \mathrm{GHz}$.

\subsubsection{Preliminary Design Considerations}

For good coupling the microstrip line must support a loosely bound mode, therefore the dimensions of the microstrip line and substrate must be small. The minimum dimensions are constrained by conductor loss, fabrication limitations and the characteristic impedance. The latter constraint corresponds to the $50 \Omega$ coaxial cables that were part of the test equipment. It was desirable not to have a $1 / 4$-wave transformer due to the accompanying radiation and surface-wave excitation. A trade-off was conducted and two microstrip lines were chosen to use as feeds for DRA arrays. The parameters for two different lines are shown in Table 4.6. The variables in Table 4.6 correspond to the diagram in Figure 4.25. The substrate material was based on Rogers 5880 $\left(\epsilon_{r}=2.16, \tan \delta=0.001\right)$. The metal trace was copper $\left(\sigma=5.88 \times 10^{7} \mathrm{~S} / \mathrm{m}\right)$. The transmission line loss was calculated from closed form expressions in [50]. 


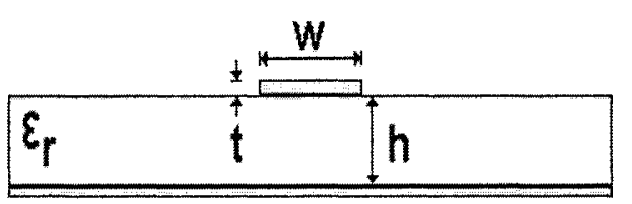

Figure 4.25: Microstrip line parameters

Table 4.6: Microstrip line dimensions

\begin{tabular}{|c|c|c|c|c|c|c|c|}
\hline Case & $\mathrm{W}(\mathrm{mm})$ & $\epsilon_{r}$ & $\mathrm{~h}(\mathrm{~mm})$ & $\mathrm{t}(\mathrm{um})$ & $Z_{o}$ & $\lambda_{g}(\mathrm{~mm})$ & $\alpha_{\text {total }}(\mathrm{dB} / \mathrm{cm})$ \\
\hline \hline$i$ & 2 & 2.2 & 0.635 & 35 & 51.7 & 7.2 & 0.0576 \\
\hline$i i$ & 0.75 & 2.2 & 0.254 & 35 & 50.4 & 7.3 & 0.0897 \\
\hline
\end{tabular}

The orientation of the DRA's with respect to the microstrip line was investigated. The two orientations considered are shown in Figure 4.26.

The $H_{x}$ and $H_{y}$ components of the microstrip line just above it are shown in Figure 4.27. The dominant component at the location of the DRA's is $H_{y}$. The dominant component of the $T E_{1 \delta 1}^{y}$ mode in the DRA is $H_{y}$, therefore this orientation was used.

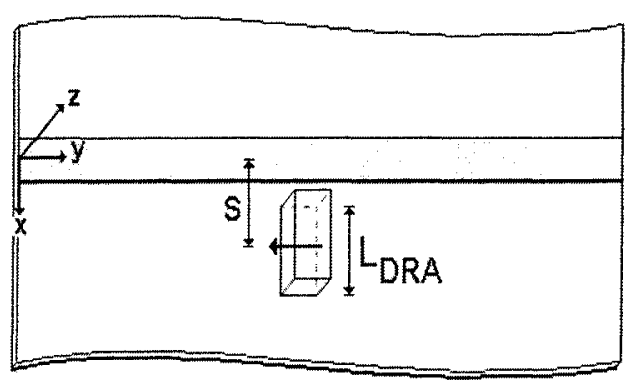

(a) $T E_{1 \delta 1}^{y}$

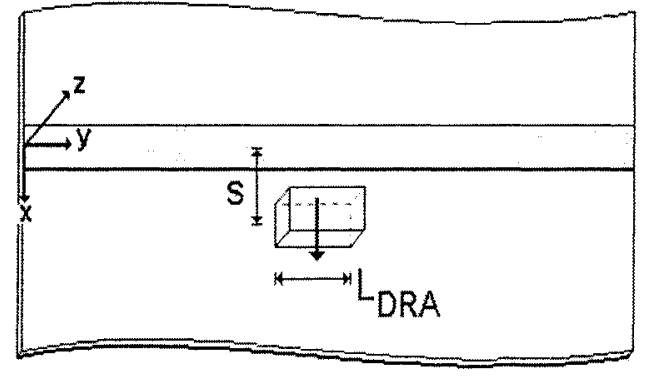

(b) $T E_{\delta 11}^{x}$

Figure 4.26: Orientations of DRA's with respect to microstrip line

The DRA's were placed on a substrate as opposed to directly on the ground 


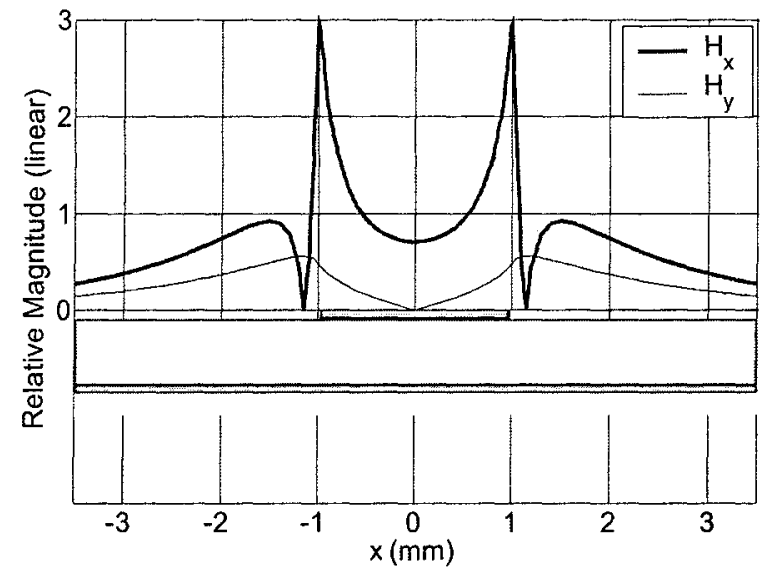

Figure 4.27: Relevant H-field components of the microstrip line

plane as was the case with the DIG fed array. This substrate affects the resonant frequency of the DRA. The combination of the substrate and the loading of the microstrip line onto the DRA resulted in disagreement between the resonant frequency compared to that predicted by the DWGM.

Preliminary simulation results showed that the DRA's had to be in contact with the microstrip line for acceptable coupling. DRA's that were near but not in contact with the microstrip generally coupled out less than $5 \%$ of the energy from the feed line. The dimensions were varied, and the relative permittivity of the DRA's was increased from 10 to 30, but the same coupling levels resulted. The primary effect of this result was that an amplitude distribution could not be achieved across the array by placing the DRA's at different distances from the transmission line at $30 \mathrm{GHz}$.

For case $i i$ in Table 4.6 the microstrip line was relatively narrow, therefore to be in contact with the line the DRA could either be located flush against 
one side, or centred on the line. The radiation patterns for the two locations were different and are shown in Figure 4.28. The dimensions of the DRA were different for the two locations to ensure resonance occurred at $30 \mathrm{GHz}$.

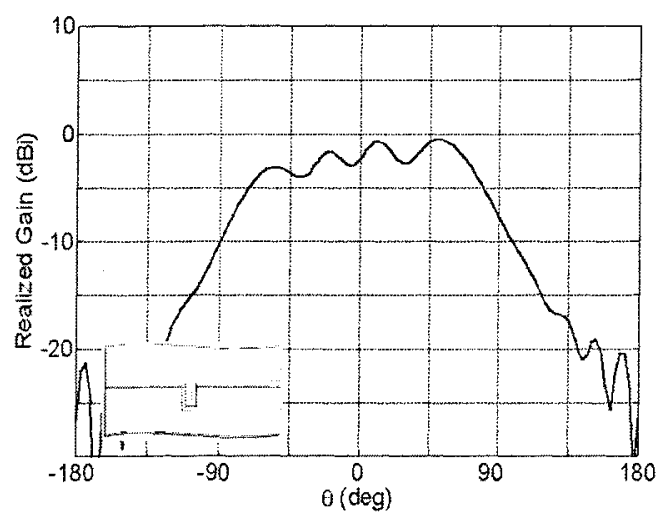

(a) DRA flush to one side of microstrip

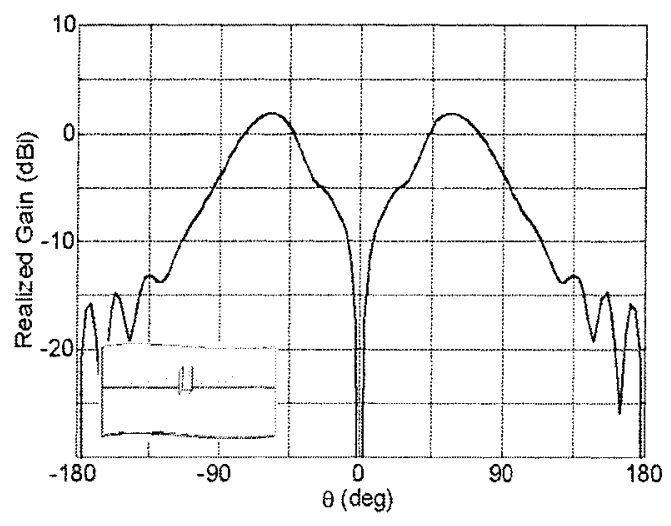

(b) DRA centred on microstrip

Figure 4.28: Radiation patterns of $E_{\theta}$ for a single DRA in the $\phi=0^{\circ}$ plane

The radiation pattern from Figure 4.28(a) is typical for a short magnetic dipole oriented parallel to the $y$-axis. The pattern from Figure 4.28(b) is typical for a short vertical dipole oriented parallel to the $z$-axis. These results indicated that the $T E_{1 \delta 1}^{y}$ mode is being excited for the DRA that is flush to the microstrip line, and the $T M_{11 \delta}^{z}$ mode for the DRA centred over the line. In this work the $T E_{1 \delta 1}^{y}$ mode was of interest since the direction of maximum radiation $\left(\theta=0^{\circ}\right)$ is the same as for the DIG fed array. 


\subsubsection{Coupling Between Microstrip and the DRA - Simulation}

An EM simulator was used to determine the power coupling characteristics between a microstrip line and a DRA. A power budget for the problem is shown in Figure 4.29. Absorbing ports were used in the simulations, which resulted in no insertion or radiation losses due to the ports.

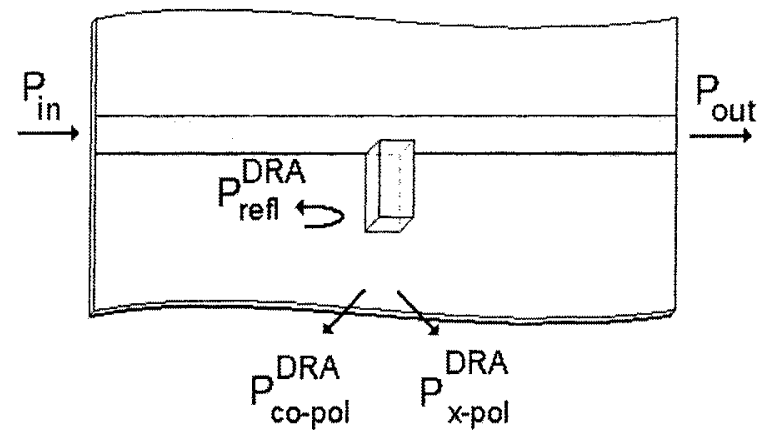

Figure 4.29: Design variables for microstrip series feed DRA array

The total radiation loss due to the DRA is calculated from (4.19).

$$
\frac{P_{c o-p o l}^{D R A}+P_{x-p o l}^{D R A}}{P_{\text {in }}}=\left(1-\frac{P_{o u t}}{P_{\text {in }}}-\frac{P_{r e f l}^{D R A}}{P_{\text {in }}}\right)
$$

The s-parameter results were used to calculate the terms on the right side of (4.19). The relationships between the s-parameters and these terms are:

$$
10 \log _{10}\left(\frac{P_{\text {out }}}{P_{\text {in }}}\right)=s_{21}[d B] \quad 10 \log _{10}\left(\frac{P_{\text {refl }}^{D R A}}{P_{\text {in }}}\right)=s_{11}[d B]
$$


The ratio of $\mathrm{x}$-pol and co-pol to total radiation was acquired from the 3-D farfield pattern. The equations that showed how the patterns were numerically integrated to calculate the ratios were shown in section 4.3 .

To be consistent with the simulated DIG arrays, two of the DRA dimensions were fixed at $1.27 \mathrm{~mm}$, and the permittivity at $\epsilon_{r}=10$. The length was varied to determine the resonant frequency. Simulations were conducted for both microstrip lines of Table 4.6. The results are shown in Table 4.7. The simulation results were recorded at $30 \mathrm{GHz}$. The length of the DRA was varied to get a range of coupling values. The radiation patterns for DRA's that are near resonance are similar to those at resonance, as shown in Figure 4.30.

Table 4.7: Summary of losses for a single DRA for various cases

\begin{tabular}{|c|c|c|c|c|c|}
\hline Case & $L_{D R A}(\mathrm{~mm})$ & $s_{11}(\mathrm{~dB})$ & $s_{21}(\mathrm{~dB})$ & $\frac{P_{c o-p o l}^{D R A}}{P_{\text {total }}^{D R A}}(\%)$ & $\frac{P_{c o-p o l}^{D R A}}{P_{\text {in }}}(\%)$ \\
\hline \hline$i$ & 2.0 & -17.3 & -0.83 & 94.9 & 14.747 \\
\hline$i$ & 2.5 & -18.1 & -0.92 & 95.55 & 16.76 \\
\hline$i$ & 3.0 & -20.1 & -0.757 & 95.57 & 14.35 \\
\hline$i$ & 3.5 & -22.1 & -0.559 & 95.11 & 10.90 \\
\hline$i i$ & 1.905 & -16.0 & -0.22 & 96.65 & 2.35 \\
\hline$i i$ & 2.54 & -14.9 & -0.65 & 98.31 & 10.48 \\
\hline$i i$ & 3.175 & -17.6 & -0.72 & 98.29 & 13.31 \\
\hline$i i$ & 3.81 & -18.8 & -0.35 & 96.64 & 6.21 \\
\hline
\end{tabular}

By varying the length of the DRA, the radiating dipole moves away from resonance and the amplitude decreases. This variation of the DRA length was used to achieve an amplitude distribution across the array. Figure 4.30(b) shows that if the length is too far away from the resonant length then the pattern deviates significantly from that of a short magnetic dipole. 


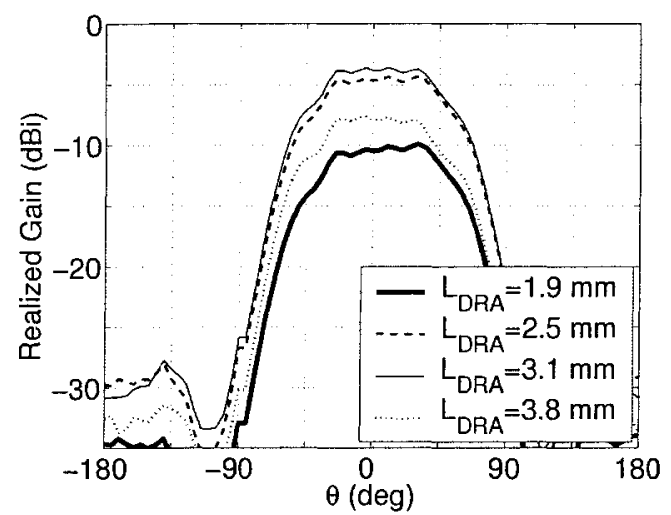

(a) $E_{\phi}$ in the $\phi=90^{\circ}$ plane

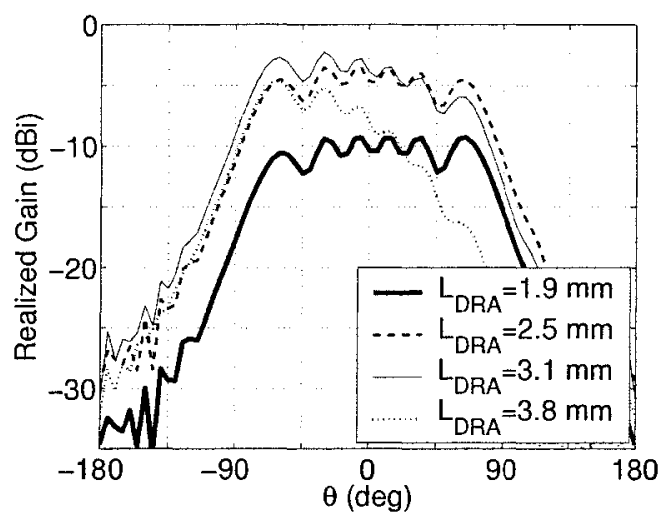

(b) $E_{\theta}$ in the $\phi=0^{\circ}$ plane

Figure 4.30: Radiation patterns for DRA's of different lengths for case $i i$

\subsubsection{Comparison to DIG Excited DRA Array}

The last column of Table 4.7 shows a small range of coupling values for each microstrip line. This limited range is not adequate to design an array with a common amplitude distribution. An array was designed, and all of the elements were identical. The microstrip line parameters correspond to case $i$ of Table 4.6 and the DRA's parameters correspond to the second row of Table 4.7. Using identical elements that are all located at the same distance from the feed line results in an array with a decaying amplitude distribution, which was introduced in section 1.2. The decay factor is a function of the coupling into the DRA and the microstrip line losses. The simulation results are shown in Figure 4.31-4.33. 


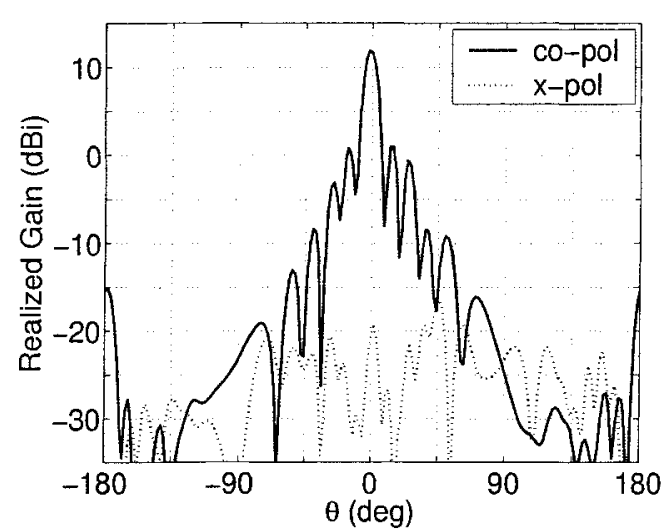

(a) $\phi=90^{\circ}$

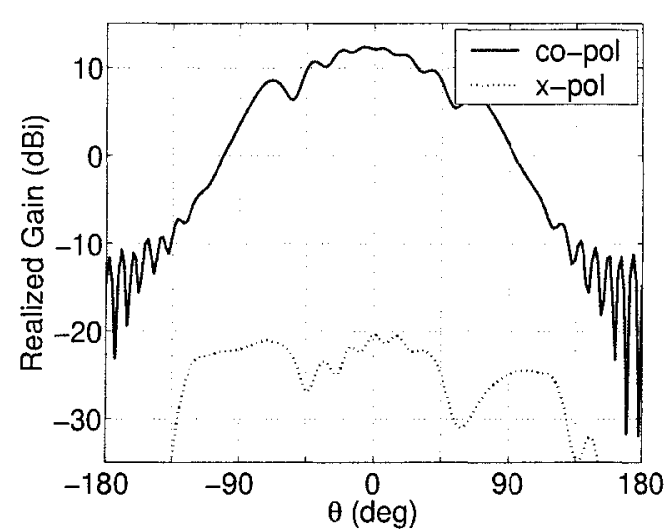

(b) $\phi=0^{\circ}$

Figure 4.31: Radiation patterns for microstrip line fed DRA array at $30 \mathrm{GHz}$

\section{Discussion of Results}

The results in Figure 4.31 show a superior x-pol level and better symmetry in the $\phi=0^{\circ}$ plane in comparison to the DIG fed arrays in the previous sections. The variation of the maximum gain over $29-31 \mathrm{GHz}$ is approximately $1.3 \mathrm{~dB}$. If this variation is acceptable then the bandwidth is only limited by frequency scanning.

The return loss is relatively large at the centre frequency. The reflections due to each of the DRA's add in phase thereby degrading the input match. The fields of the DIG were similar to those of the DRA therefore there was less reflection for the DIG fed DRA array.

The major drawback of the microstrip line fed DRA array was the inability to achieve a specific amplitude distribution at high frequencies. The simplicity of fabrication and connecting to a coaxial cable made the microstrip line a good 


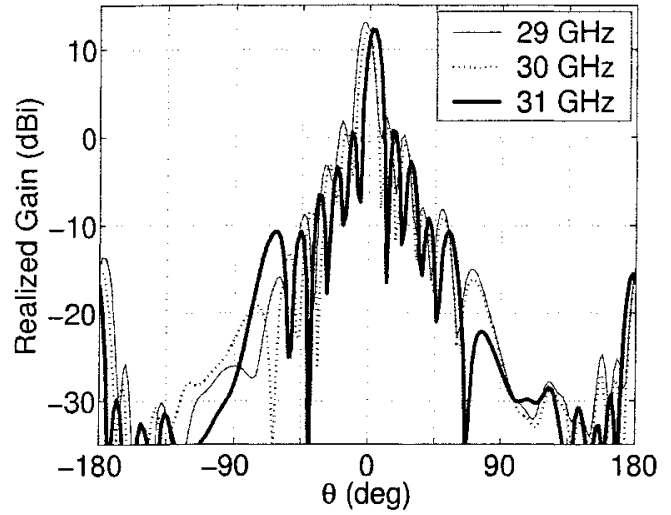

(a) $\phi=90^{\circ}$

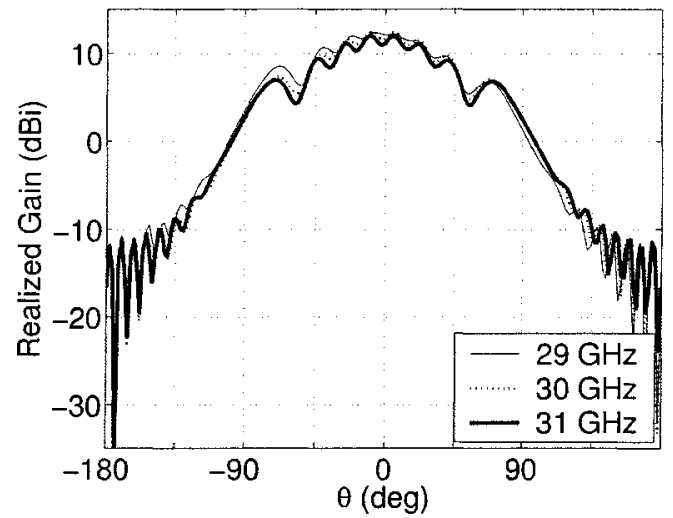

(b) $\phi=0^{\circ}$

Figure 4.32: Co-pol radiation patterns for microstrip line fed DRA array

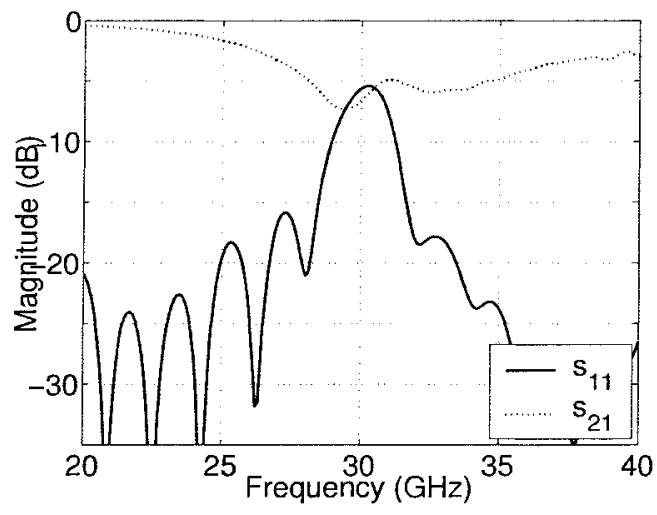

Figure 4.33: S-parameters for microstrip line fed DRA array

candidate for a series fed DRA array. This feed was used in section 5.3 for an investigation of DRA phase shift properties since it was more efficient to simulate in Empire. 


\section{Chapter Summary}

The purpose of this chapter was to show that CMT could be applied to approximate the coupling between a DIG and a DRA. This approximation was validated with simulations. The design rules were created to govern the design parameters of the DIG and DRA's of a DIG fed DRA array. The design rules and CMT were applied to design several different arrays with specific amplitude distributions. The DIG fed DRA array was compared to the microstrip line fed DRA array. 


\section{Chapter 5}

\section{Array Performance Improvements}

Several modifications have been proposed for the DRA array to improve its performance. The main beam was tilted away from the $z$-axis in all of the DIG fed arrays that were designed in chapter 4 . Methods that add symmetry with respect to the $z$-axis have been investigated to bring the maximum gain towards the $\theta=0^{\circ}$ direction. A modification has been introduced for the rectangular DRA that reduces the $\mathrm{x}$-pol by suppressing undesired radiating modes. The frequency scanning phenomenon inherent to non-resonant series fed arrays is undesirable for some applications. Design rules have been introduced to design DRA arrays that reduce the magnitude of the main beam scanning with frequency. 


\subsection{Maximizing DRA Gain at $\theta=0^{\circ}$}

Preliminary simulations showed that the close proximity of the DIG to the DRA caused the pattern to deviate from that of a short magnetic dipole. The degradation to the ideal pattern is shown in Figure 5.1 for an arbitrary geometry. The goal of this section was to devise a method to ensure the maximum gain for the DRA occurred at $\theta=0^{\circ}$.

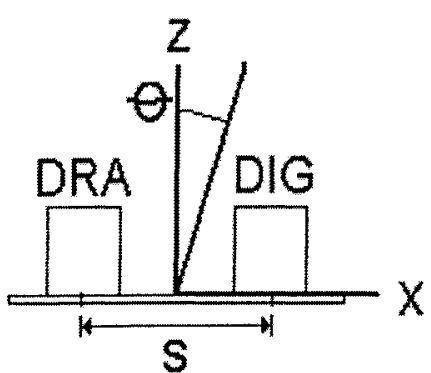

(a) DIG and DRA cross-section

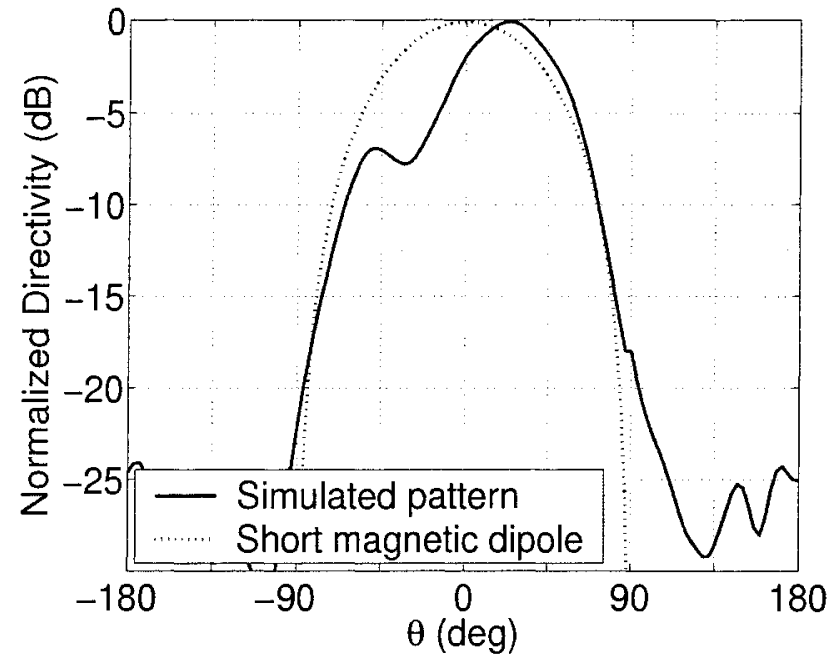

(b) Ideal and simulated patterns in the $\phi=0^{\circ}$ plane

Figure 5.1: Effect of DIG loading on radiation pattern

\subsubsection{Parasitic DRA's}

The addition of a dielectric material near a radiating element tends to alter the radiation pattern. This effect is observed in the case in Figure 5.1(a) as the DIG tends to tilt the main beam away from the $\theta=0^{\circ}$ axis. A second DRA was placed beside the original DRA to add symmetry in the $x z$-plane 
$\left(\phi=0^{\circ}\right)$. This configuration is shown in Figure 5.2. It was hypothesized that the symmetry that the additional DRA introduces in the $\phi=0^{\circ}$ plane would tilt the direction of maximum radiation beam back towards $\theta=0^{\circ}$.

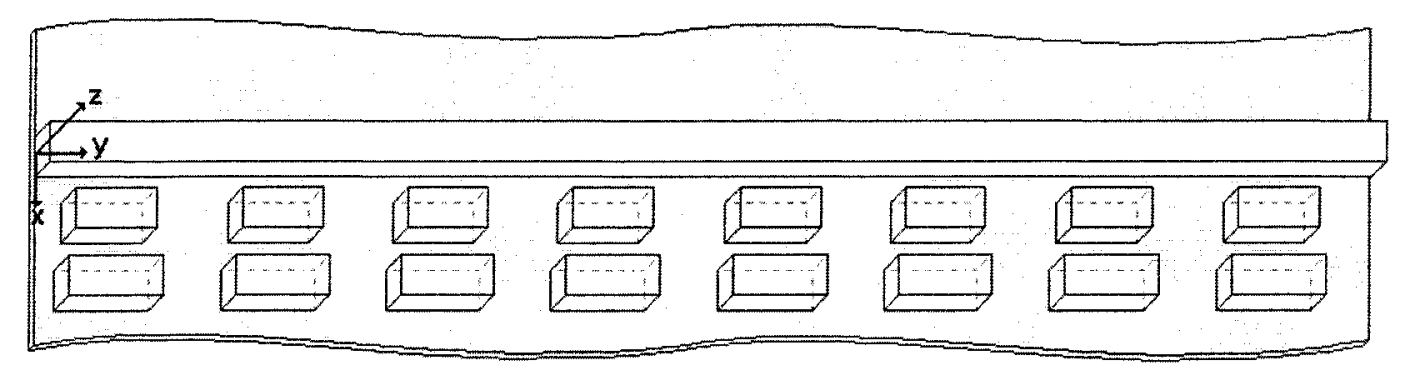

Figure 5.2: Lattice with parasitic DRA's

As an alternative to using additional DRA's for adding symmetry, two other options were explored and are shown in Figure 5.3. Figure 5.3(a) shows the addition of a second DIG, but this was not used for two reasons. First, the two DIG's make a directional coupler, therefore significant power coupling would occur between the two DIG's. Second, the space between each DRA and the original DIG varies, therefore not all of the elements would be centred between the two DIG's. This would not introduce the desired symmetry in the $\phi=0^{\circ}$ plane. Another option that was declined was the use of a slab, shown in Figure 5.3(b). The slab could be machined so that the air gap between each DRA and the DIG would be equal to the gap between that DRA and the slab. It was found that a significant amount of power was coupling into the slab, thereby degrading the gain of the array.

The cross-section of the DIG and a DRA in Figure 5.2 is similar to three parallel DIG's. The analysis from section 4.2 was based on two parallel DIG's, 


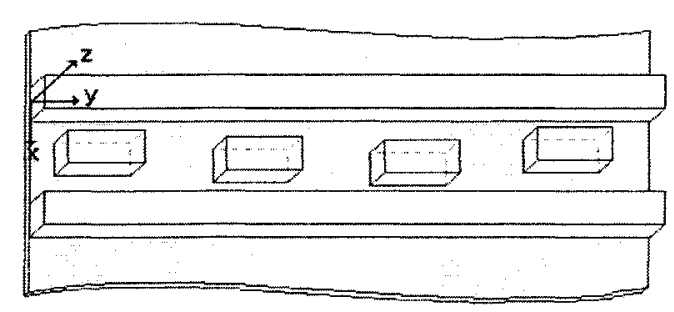

(a) Second DIG

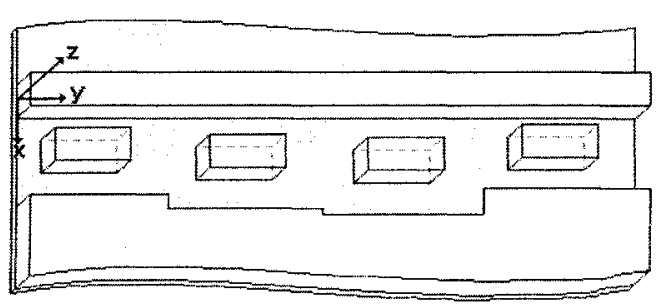

(b) Slab

Figure 5.3: Failed options for symmetric loading

so it could not be directly applied to this case. The additional DRA loads the original, consequently increasing the resonant frequency of the original DRA. The parasitic DRA adds symmetry to the original DRA in the $\phi=0^{\circ}$ plane, but this symmetry deteriorates as $\theta$ departs from $0^{\circ}$. The useful range of $\theta$ may be improved by increasing the length of the parasitic DRA, but is limited by power coupling from the DIG into this DRA.

Simulations were run to determine the resonant length of the DRA with a parasitic DRA present. The simulations were repeated for different DIG-DRA spacings $(s)$. The simulated radiation pattern for a single pair of DRA's are shown in Figure 5.4. In this figure the gain has been normalized by the maximum gain for the case when $s=2.5 \mathrm{~mm}$. The patterns in Figure 5.4 show that the DRA's maximum gain has been moved to $\theta=0^{\circ}$ for small values of $s$.

The radiation patterns for different values of $s$ change significantly. In an array environment, this translates into non-identical elements with different radiation patterns. Basic array theory cannot be applied to this type of array since the required amplitude distribution depends on the excitation of each 


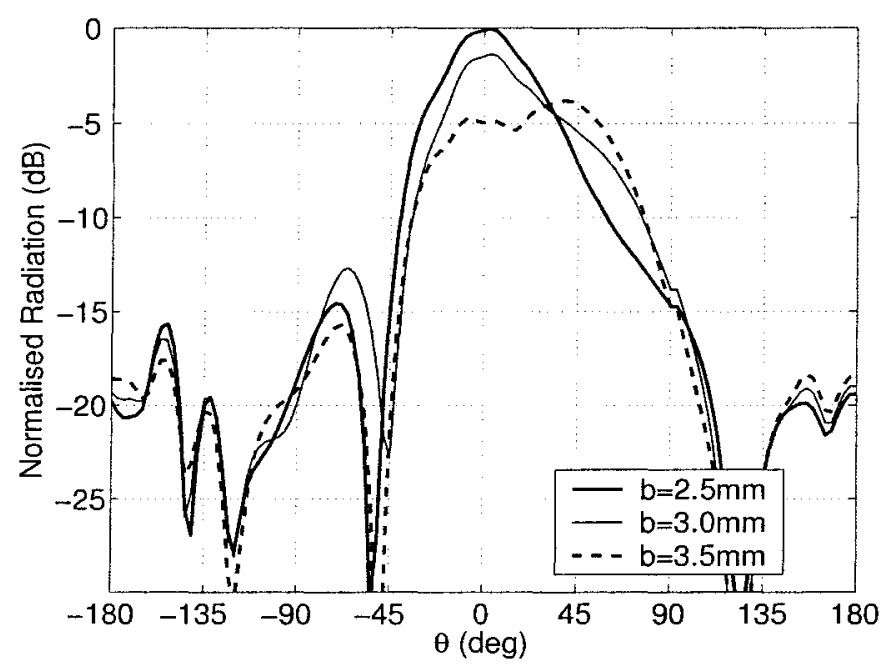

Figure 5.4: Radiation patterns for parasitically loaded DRA in the $\phi=0^{\circ}$ plane

element as well as the relative directivity of each element in the array plane. The additional DRA's are useful in an array where a specific amplitude distribution is not required since all elements can be located at the same distance from the DIG.

\subsubsection{Staggered DRA's}

A configuration that adds some symmetry in the $\phi=0^{\circ}$ plane with respect to $\theta=0^{\circ}$ was accomplished by alternating the side of the DIG that sequential DRA's were located. As a comparison, this configuration and the nonalternating one are shown in Figure 5.5. This type of array is similar to two parallel linear arrays. The problem with this design is that the elements have different element patterns depending on which side of the DIG they are on. 
The beam is tilted off the $\theta=0^{\circ}$ axis in one direction for elements on one side, and the opposite direction for those on the other side. The product of these element patterns with the array factor is a heart shaped pattern in the $\phi=0^{\circ}$ plane, which is shown for an 8-element array in Figure 5.6. Staggering the DRA's was unsuccessful at maximizing the gain at $\theta=0^{\circ}$.

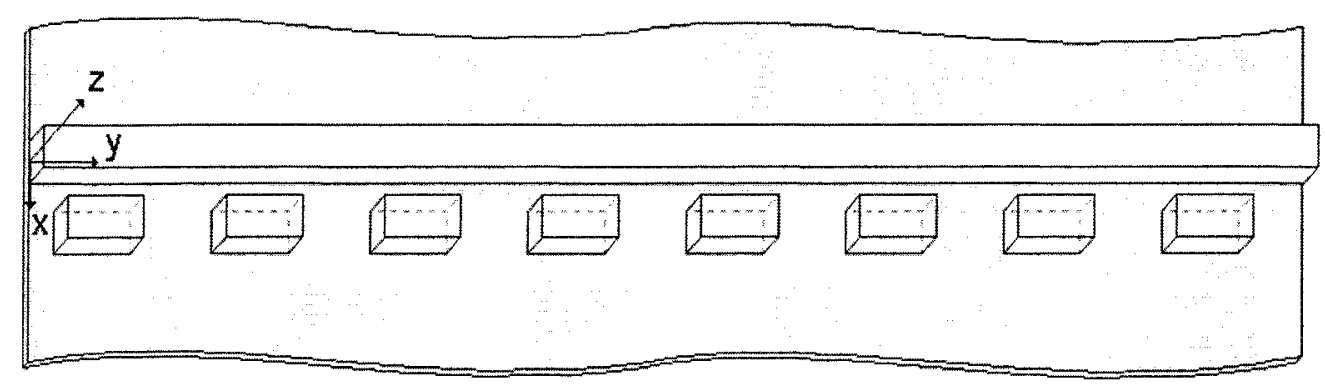

(a) Original lattice

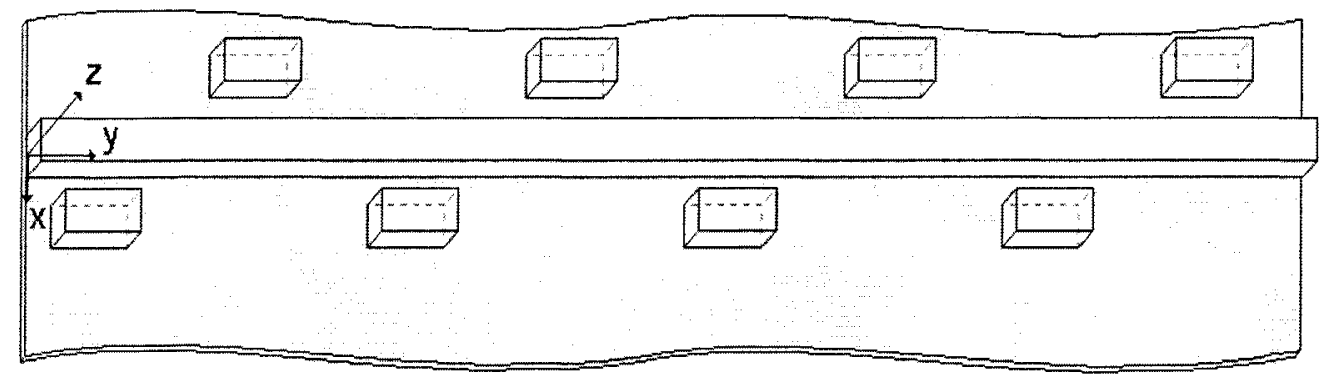

(b) Staggered lattice

Figure 5.5: Lattices of 8-element arrays

\subsubsection{Different Cross Sections Between DRA and DIG}

To reduce the interference that the DIG causes to the DRA it is desirable to reduce the dimensions and permittivity of the DIG. The limitations on minimum values for these parameters was discussed in section 3.2. The cross-section of 


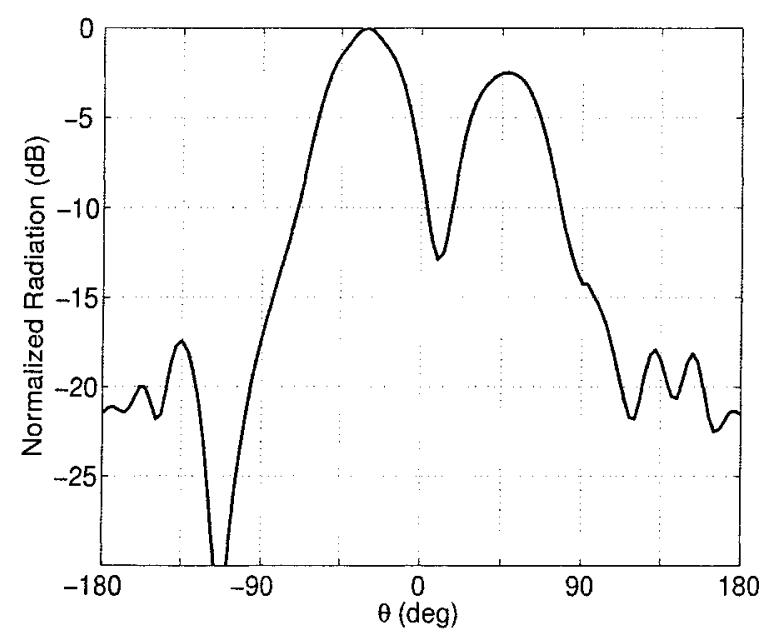

Figure 5.6: Radiation pattern for staggered DRA array in $\phi=0^{\circ}$ plane

the DRA and DIG was chosen to be identical so that only the propagation constants were required to apply CMT to approximate the coupling. Asymmetric DIG couplers have also been analyzed using CMT, but since there is no plane of symmetry the modes are classified as even-like or odd-like [51]. The main differences compared to symmetric couplers are a shorter coupling length, and the lack of being able to transfer $100 \%$ of the energy between the guides. The coupling length $\left(L_{c}\right)$ is calculated in the same manner as in section 4.2 , but an addiontal factor $(1-\delta)$ is inserted into (4.1) to account for the maximum power transfer.

$$
\frac{P_{2}}{P_{\text {in }}}=(1-\delta) \sin ^{2}\left(\frac{y}{L_{c}} \frac{\pi}{2}\right)
$$

The summation of the modes was used for identical DIG's to demonstrate how $100 \%$ of the power will transfer from the input guide to the coupled guide. The summation of modes for the asymmetric case shows how $100 \%$ of the power cannot be transferred. The fundamental even and odd modes are shown in 

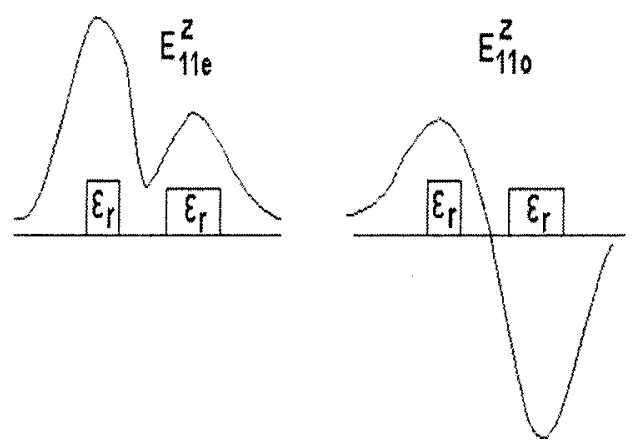

Figure 5.7: $E_{z}$ component of even and odd modes for asymmetric DIG's Figure 5.7.

The summation of the mode profiles are shown in Figure 5.8 for two nonidentical DIG's. At the input of the coupler all of the power is in one of the DIG's therefore the even mode must be multiplied by some factor $m$ so that the summation of the amplitudes of the mode profiles meets this input condition. When the modes are $180^{\circ}$ out of phase, then the summation of the mode profiles is that shown in Figure 5.8(b). In this figure not all of the power is in the coupled guide, therefore $100 \%$ power transfer does not occur. For the DRA array, it is not necessary to transfer $100 \%$ of the power into one DRA, therefore a DRA with a different cross-section to the DIG is an option. DIG fed DRA arrays that have different cross-section dimensions and permittivities with regards to Figure 4.9 have already been designed [7]. To apply CMT the $\delta$ factor must be calculated, but requires the fields of the even and odd modes of the equivalent DIG coupler. A design requiring the calculation and weigthing of the modal fields is more complex than calculating just the propagation constants. This type of antenna configuration may result 


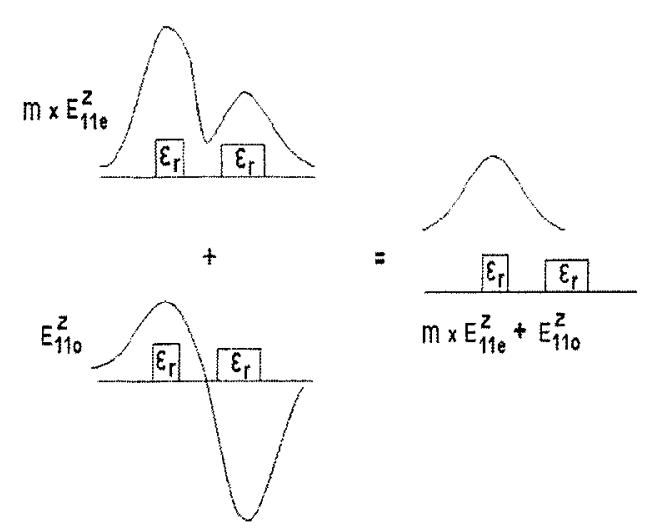

(a) Modes in phase

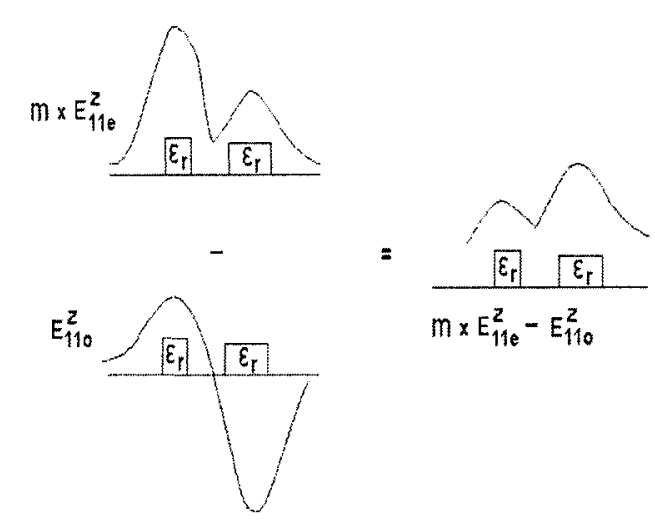

(b) Modes $180^{\circ}$ out of phase

Figure 5.8: Summation of even and odd modes for asymmetric coupler in an antenna with improved performance, but will be at the expense of design complexity.

\subsection{Reduction of Cross-Polarization}

There were two main contributors to radiated cross-polarized (x-pol) energy: the DIG feed and the excitation of higher order modes in the DRA. The input probe radiates similarly to a vertical electric dipole, which has a null in the in the $\theta=0^{\circ}$ direction. For an array with a main beam near the broadside direction, the $\mathrm{x}$-pol due to the input probe should be significantly below the main beam. The x-pol radiation pattern of the input probe is shown in Figure $3.14(d)$.

Due to the hybrid nature of the fields inside the DIG and the low permittivity of the DRA, modes other than the $T E_{\delta 11}^{x}$ were excited in the DRA. The 
main effects of these additional modes were interference with the co-polarized (co-pol) radiation pattern and contributions to x-pol radiation. A method of suppressing these additional modes has been developed that is unique to the way that the DRA is fed in this work. The DRA is fed along its entire length by the DIG, so inserting a metal square inside the DRA, normal to the $y$-direction, will not severely affect the power distribution between the two halves of the DRA. This modification is shown in Figure 5.9. This metal square suppresses the $T E_{1 \delta 1}^{y}$ magnetic dipole mode and the $T M_{11 \delta}^{z}$ electric dipole mode. These modes were also suppressed in earlier work done by Tam and Murch [52], but at the expense of a decrease in the radiation space by half. The radiation patterns of an array with and without the metal shorts are shown in Figures 5.10(a) and 5.10(b) at $30 \mathrm{GHz}$. These patterns show a maximum deviation of $0.6 \mathrm{~dB}$ to the co-pol gain and approximately $5 \mathrm{~dB}$ improvement to the $\mathrm{x}$-pol gain over a relatively wide range.

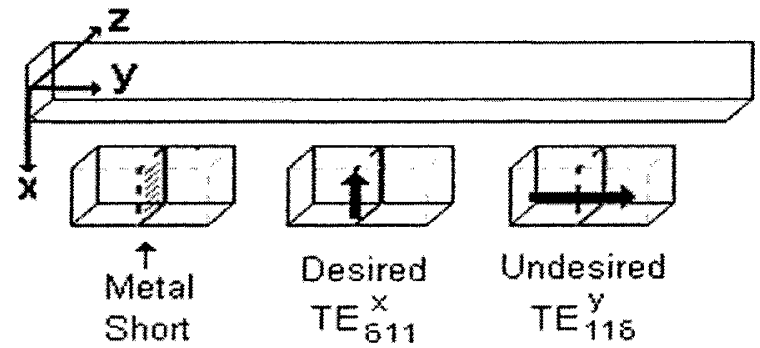

Figure 5.9: Metal shorts

These patterns only show the effect in the principle plane, so to demonstrate the effectiveness of the shorts an analysis of the 3-D far-field radiation pattern was conducted. The effect of the shorts were investigated on one of the 


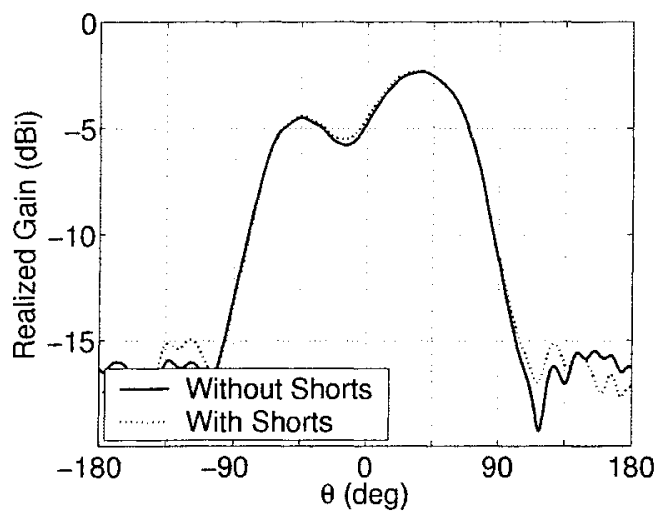

(a) Co-pol gain

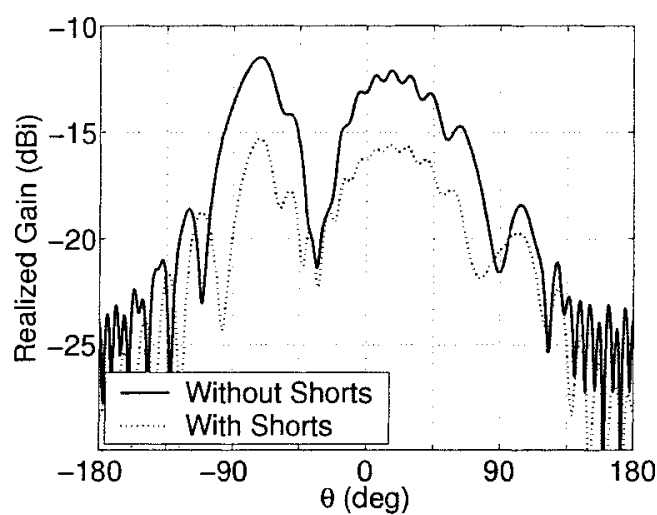

(b) X-pol gain

Figure 5.10: Radiation pattern in $\theta=0^{\circ}$ plane with x-pol reduction

arrays designed in section 4.4.2. The array had a Chebyshev amplitude distribution across the elements, and the element spacing was $0.8 \lambda_{o}=1.25 \lambda_{g}$. The radiation from the coaxial-to-DIG transition was subtracted from the total radiation pattern. At $30 \mathrm{GHz}$ the maximum co-pol gain of the array with the shorts was about $1 \mathrm{~dB}$ larger than the array without the shorts. Without the metal shorts, some of the undesired radiating modes contribute to the co-pol radiation, therefore can constructively or destructively interfere with the copol patter. For this array the maximum co-pol gain improved by adding the short, therefore the undesired radiating modes were destructively interfering in the direction of maximum gain. The maximum $\mathrm{x}$-pol gain of the modified array had improved by about $2 \mathrm{~dB}$. At $31 \mathrm{GHz}$ the maximum x-pol gain of the shorted array was about $3.5 \mathrm{~dB}$ better than the original array.

An array with a Taylor amplitude distribution was designed in section 4.4.3 at $60 \mathrm{GHz}$. The shorts were added to this array, and the results in the $\mathrm{H}$-plane of the main beam are shown in Figure 5.11. Over most of the angular range the 
$\mathrm{x}$-pol level has been reduced. In the $\theta=0^{\circ}$ direction the $\mathrm{x}$-pol had improved by almost $10 \mathrm{~dB}$, and the co-pol gain by $1 \mathrm{~dB}$.

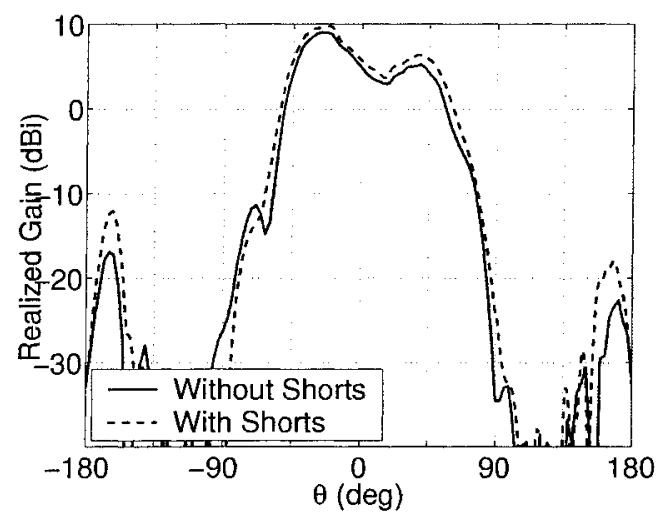

(a) Co-pol gain

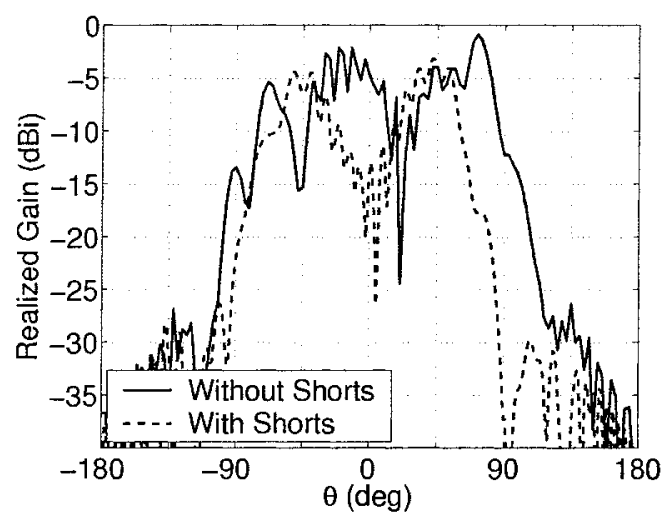

(b) X-pol gain

Figure 5.11: X-pol reduction for Taylor array at $60 \mathrm{GHz}$

These results show that the introduction of the shorts does improve the $\mathrm{x}$-pol level of the array. The patterns show that the direction of the maximum x-pol gain changes due to the addition of the shorts. This effect should be taken into account to meet $\mathrm{x}$-pol specifications in specific directions.

\subsection{Reduction of Frequency Scanning}

Frequency scanning is a characteristic of an array with a travelling wave type feed. The main beam direction is frequency dependent. Other methods have been reported to reduce the frequency scanning in series fed arrays. The feed line can be made resonant by open or short-circuiting one end [49]. This configuration is bandwidth limited since the input is matched at the operating 
frequency, and the distance between maxima of the feed line energy will correspond to the element locations at the operating frequency. A second method of reducing frequency scanning is by using phase shifters between the feed line and each element. These phase shifters can be controlled to counteract the frequency dependent phase progression in the feed line. Phase shifters increase the complexity of the array, especially for the arrays in this work since the radiating elements are not directly connected to the feed line.

\section{Microstrip Series Feed}

For a linear array of identical radiators fed by a microstrip line, the phase difference between the elements is a function of frequency and the lines properties. From array theory, the relationship between the phase progression between elements $(\beta)$ and the direction of the main beam in the array plane with respect to the broadside direction $\left(\theta_{o}\right)$ is given by:

$$
-\beta=k_{o} d \sin \theta_{o}
$$

The phase progression for a microstrip-fed array with an element spacing $d$ is:

$$
\beta=-\frac{2 \pi}{\lambda_{g}}\left(\lambda_{g}-d\right)
$$

The term $\lambda_{g}$ is the guided wavelength of the microstrip line as is approximated using (5.4). 


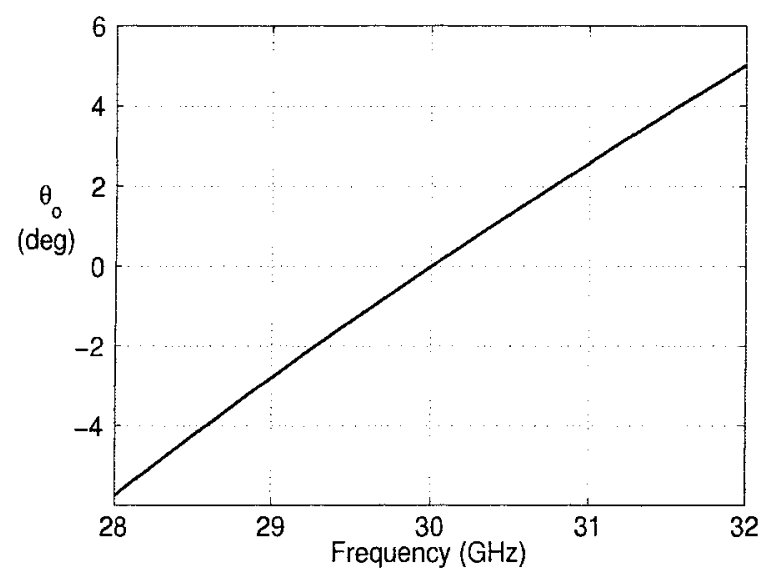

Figure 5.12: Frequency scanning properties of a linear array

$$
\lambda_{g}=\frac{\lambda_{o}}{\sqrt{\epsilon_{e f f}}}
$$

The relative effective permittivity $\left(\epsilon_{e f f}\right)$ for the microstrip line is assumed to be constant over the operating bandwidth. Manipulating (5.2)-(5.4) gives the relationship:

$$
\sin \theta_{o}=\frac{\sqrt{\epsilon_{e f f}}}{d}\left(\frac{\lambda_{o}}{\sqrt{\epsilon_{e f f}}}-d\right)
$$

Equation (5.5) shows the dependency of the main beam angle on frequency. Figure 5.12 shows this relationship for the microstrip line corresponding to case $i$ in Table 4.6 , with $d=7.5 \mathrm{~mm}$.

Phase shifters could be added to the elements of an array fed by this microstrip line as shown in Figure 5.13. The requirement of the phase shifters would be to counteract the phase progression of the microstrip line. The actual phase shifts required $\left(\phi_{n}\right)$ is given by (5.6). This equation shows that $\phi_{n}$ is frequency 


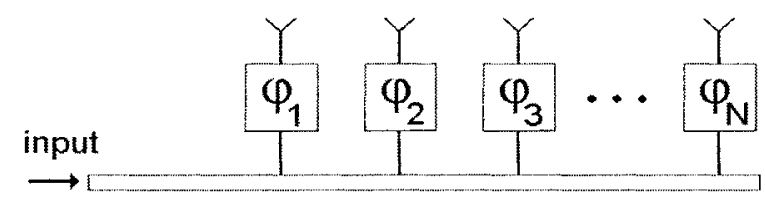

Figure 5.13: Series-fed array employing phase shifters for each element dependent, and due to the $n$ term, it also depends on the element.

$$
\begin{aligned}
\phi_{n} & =-(n-1) \beta \\
& =2 \pi(n-1)\left(1-\frac{d \sqrt{\epsilon_{e f f}}}{\lambda_{o}}\right)
\end{aligned}
$$

\section{DRA's as Phase Shifters - Theory}

A DRA has an inherent phase shift that is frequency dependent. The phase delay characteristics are based on the geometry, material properties and the type of loading due to the feeding mechanism. The DRA can be considered as a bandpass filter between the transmission line and free space. The frequency dependent phase shift is demonstrated by considering the phase response for several bandpass filters, as shown in Figure 5.14. At any frequency the slopes of all of the curves have the same sense, but different magnitudes.

Equation 5.6 shows that for a specific element ( $n$ is fixed) the required phase shift must increase as frequency decreases, and vice versa. This is the relationship that is oberserved in Figure 5.14. If the phase response for each DRA could satisfy (5.6) over some bandwidth, then the array would not scan with frequency. 


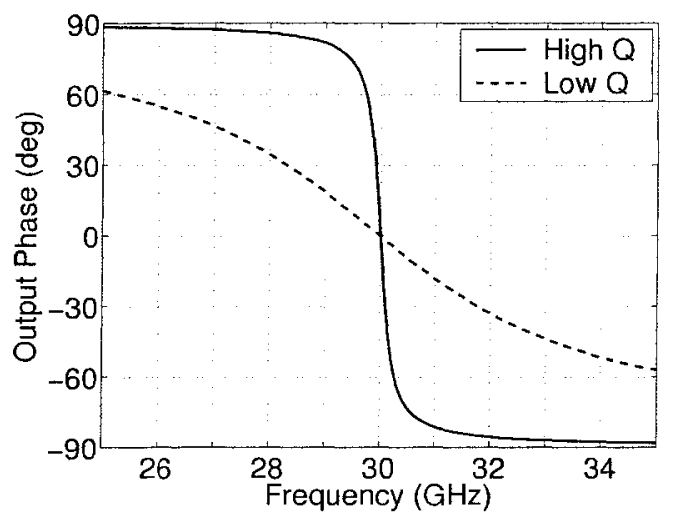

(a) $f_{o}=30 \mathrm{GHz}$

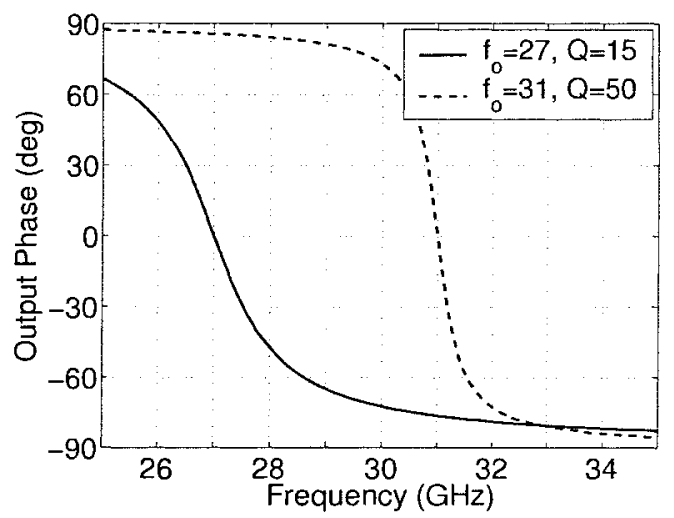

(b) Arbitrary resonators

Figure 5.14: Typical output phase for bandpass filters with $0^{\circ}$ input phase

Design guidelines were generated to reduce the frequency scanning of a seriesfed array. The operating bandwidth is defined by the lower frequency $\left(f_{l}\right)$ and the higher frequency $\left(f_{h}\right)$. The power radiated by the elements of an array must be in phase in the far-field in the direction of the main beam at $f_{l}$ and $f_{h}$. The terms $\lambda_{g_{l}}$ and $\lambda_{g_{h}}$ refer to the guided wavelength of the transmission line at the lower and higher frequency.

\section{At the lower frequency:}

To reduce frequency scanning different DRA's were used in an array, but the inherent phase shift of different DRA's is generally not equal at a specific frequency. This result is demonstrated by Figure 5.14(b) since the two curves are not overlapping. If a fixed element spacing $(d)$ was applied to an array of different DRA's, then the radiated phase of each element may significantly vary. The location of each element deviated from that of a linear array to account for the differences in the phase responses. The phase difference was 
calculated at $f_{l}$, and relative to the output phase of the first element in the array $\left(\phi_{n_{l}}-\phi_{1_{l}}\right)$. Equation (5.7) gives the offset of each element $\left(\Delta_{n}\right)$ relative to the equivalent linear array with an element spacing based on the main beam direction. A positive value of $\Delta_{n}$ corresponds to shifting the element closer towards the input. The input direction is shown in Figure 5.13.

$$
\Delta_{n}=\left(\phi_{1_{l}}-\phi_{n_{l}}\right) \frac{\lambda_{g_{l}}}{2 \pi}
$$

The actual spacing between the first element $(n=1)$ and each of the DRA's is given by (5.8). The variable $d_{n}$ is defined in Figure 5.15.

$$
d_{n}=(n-1) \lambda_{g_{l}}+\Delta_{n}
$$

At the higher frequency:

The magnitude of the phase progression along the array increases with frequency, but the transfer function phase response of the DRA's decreases (5.14). The input phase at each element relative to the first element is $d_{n} \frac{2 \pi}{\lambda_{g_{h}}}$. To counteract the variation in input phase, each element must have an inherent shift equal in magnitude but opposite in sign. The required phase shift relative to that of the first element is given by (5.9).

$$
\phi_{1_{h}}-\phi_{n_{h}}=d_{n} \frac{2 \pi}{\lambda_{g_{h}}}-2 \pi(n-1)
$$


Equations (5.7)-(5.8) are substituted into (5.9) to get:

$$
\phi_{1_{h}}-\phi_{n_{h}}=\frac{\lambda_{g_{l}}}{\lambda_{g_{h}}}\left[2 \pi(n-1)+\left(\phi_{1_{l}}-\phi_{n_{l}}\right)\right]-2 \pi(n-1)
$$

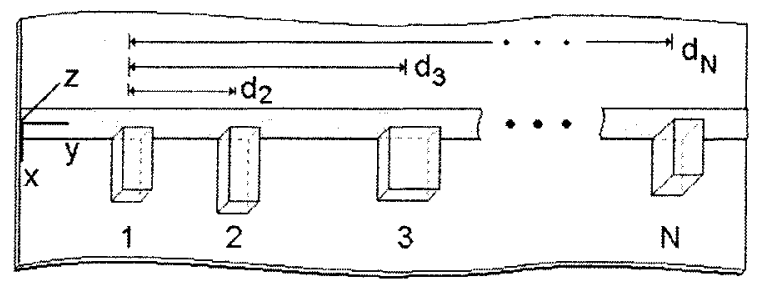

Figure 5.15: General diagram of N-element array

If DRA elements can be chosen to satisfy (5.10), then the array's main beam will point in the same direction at $f_{l}$ and $f_{h}$.

\section{DRA's as Phase Shifters - Design}

The Q-factor and resonant frequency are sufficient to calculate the value of $\phi_{n_{l}}$ and $\phi_{n_{h}}$ for a DRA. Approximations for these parameters are described in [10] for isolated DRA's. To validate this idea more accurate values of $\phi_{n_{l}}$ and $\phi_{n_{h}}$ were required. FDTD simulations were conducted using Empire for a single DRA fed by a microstrip line, as shown in Figure 4.29. The dimensions and permittivity of the DRA were varied and the far-field phase was recorded directly above the DRA $\left(\theta=0^{\circ}\right)$ for each case. The phase was normalized to the input phase at the DRA for each frequency point. The phase results were recorded from 29 to $31 \mathrm{GHz}$ at $0.5 \mathrm{GHz}$ intervals. A routine was coded in MATLAB to pick the DRA's that best satisfy (5.10). 
An 8-element array was designed to minimize the frequency scanning from 29 to $30 \mathrm{GHz}$. The results were compared to an 8-element array of identical elements all fed in phase. The simulated radiation patterns are shown in Figure 5.16 for three discrete points. The scan angle is plotted against frequency over a range that extends past the intended band. The results for the beam peak location for both arrays is shown in Figure 5.17. The dimensions and permittivity of the DRA's are shown in Table 5.1. The parameter $L_{D R A}$ is defined in Figure 4.26(a), $h_{D R A}$ is the height in the z-dimension, and $w_{D R A}$ is the width in the y-dimension. The microstrip parameters correspond to case $i$ of Table 4.6 .

Table 5.1: DRA parameters for array with reduced frequency scanning

\begin{tabular}{|c|c|c|c|c|}
\hline DRA & $\epsilon_{r}$ & $L_{D R A}(\mathrm{~mm})$ & $h_{D R A}(\mathrm{~mm})$ & $w_{D R A}(\mathrm{~mm})$ \\
\hline \hline 1 & 20 & 2.54 & 1.91 & 1.27 \\
\hline 2 & 12 & 3.81 & 2.54 & 1.27 \\
\hline 3 & 10 & 4.45 & 1.91 & 2.54 \\
\hline 4 & 10 & 3.81 & 1.91 & 1.27 \\
\hline 5 & 6 & 3.81 & 2.54 & 1.27 \\
\hline 6 & 12 & 3.81 & 1.27 & 1.27 \\
\hline 7 & 12 & 3.81 & 1.27 & 1.27 \\
\hline 8 & 12 & 3.81 & 1.27 & 1.27 \\
\hline
\end{tabular}




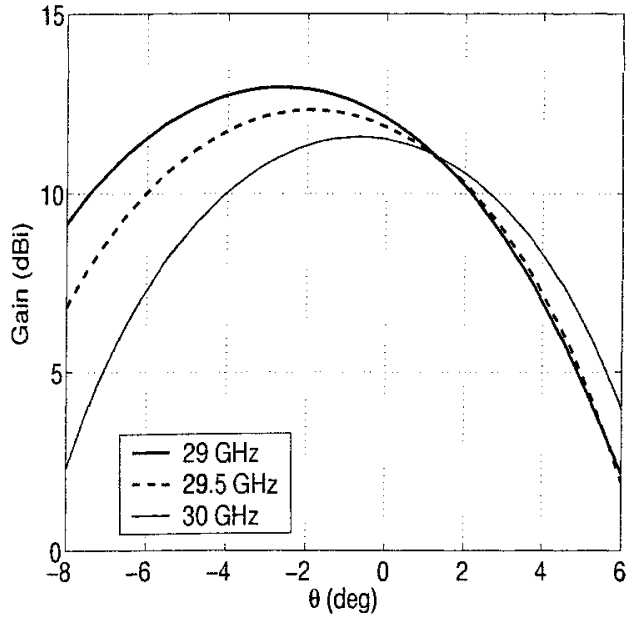

(a) Array of identical elements

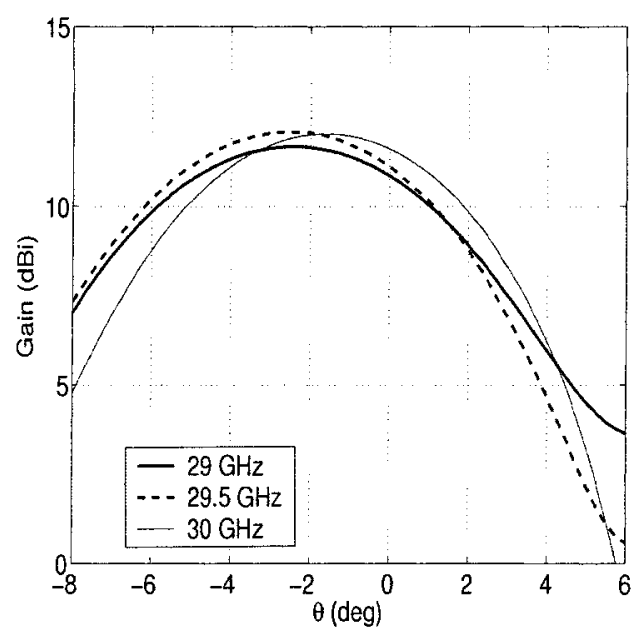

(b) Applied criteria for minimum frequency scan

Figure 5.16: Gain radiation patterns for microstrip fed DRA arrays

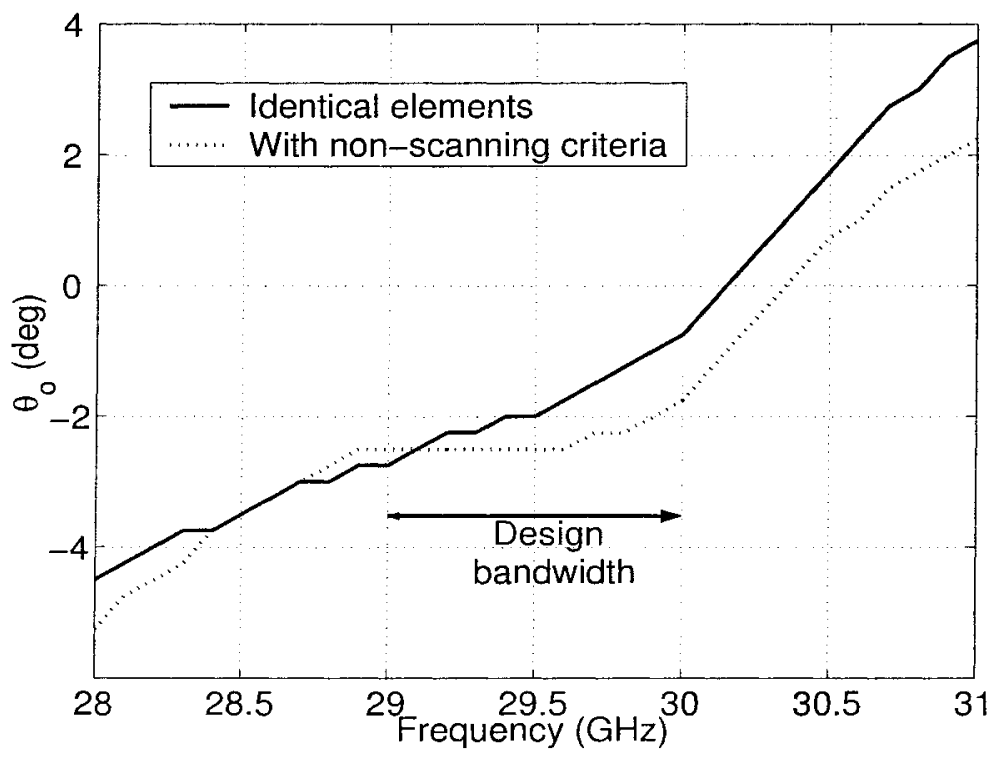

Figure 5.17: Beam peak location versus frequency 
The gain bandwidth was investigated by plotting the gain in a specific direction as a function of frequency. The gain was considered in the array plane in the $\theta=-2^{\circ}$ direction, which corresponded to the direction of the main beam at the centre frequency. The gain curve for the array designed with reduced frequency scanning is plotted against that curve for the array of identical elements. The gain for the modified array shows less variation of gain over the bandwidth than for the case of identical elements. This improvement was attributed to the reduced frequency scanning and the variation of element spacing for the modified array.

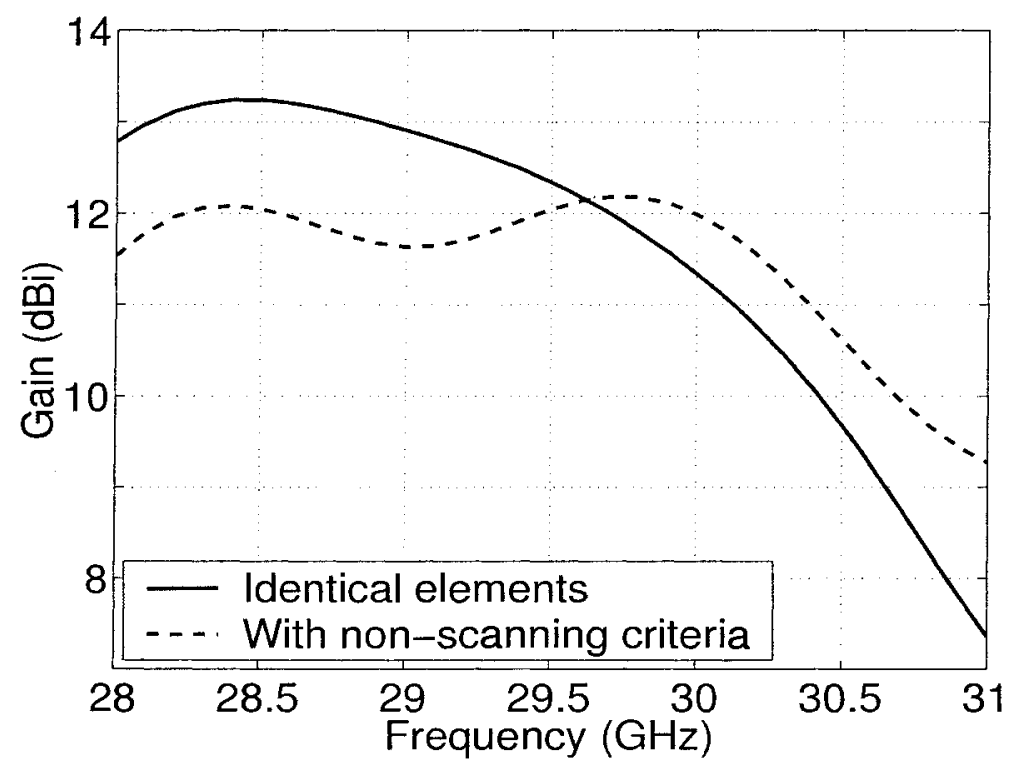

Figure 5.18: Gain in the $\phi=90^{\circ}$ plane in the $\theta=-2^{\circ}$ direction 


\section{Chapter Summary}

Several array improvements were proposed. Parasitic DRA's were applied to ensure the maximum directivity of the element pattern of a DRA in the vicinity of a DIG is at $\theta=0^{\circ}$. Metal shorts were added to the DRA's to reduce the x-pol. Lastly, a set of guidelines were introduced to design a non-resonant series fed array that scans less with frequency than a linear array with identical elements and uniform spacing. 


\section{Chapter 6}

\section{Conclusion}

Design guidelines have been established to design a DIG fed DRA analytically. These guidelines were used to design this type of array with standard amplitude and phase distributions. A microstrip fed DRA array was investigated for comparison to the DIG case. At high frequencies significant amounts of power coupled into the DRA's from the microstrip line, but there was not as much range in coupling values as with the array fed by a DIG. Several performance enhancing modifications were introduced.

\section{Design Guidelines}

The DIG dimensions and permittivity should be picked to ensure that the DIG supports a loosely bound mode, but not too loose. The ratio $v_{p} / c$ was in the range 0.7-0.9 throughout this thesis work. The dielectric waveguide 
model can be used as a first order approximation to calculate the resonant length of the DRA. If the DIG's surface-wave is too loosely bound, then the length of the DRA will be so long that the conditions for grating lobes will be satisfied. Furthermore, if the elements are very long then the physical gap between them will be small and mutual coupling will become significant. The parameters required from the selected DIG are the losses and characteristic parameter. Coupled mode theory (CMT) is then applied to the DIG and DRA to approximate the power coupled into the DRA as a function of the spacing between them. The required amount of power to couple into each DRA is a function of the required amplitude distribution. The application of CMT worked best for DIG's that supported a loosely bound surface-wave.

\section{Array Improvements}

One consequence of the DIG in proximity to the DRA's is a tilt of the maximum gain away from $\theta=0^{\circ}$. If a specific amplitude distribution is not required, then the maximum gain can be pointed in the $\theta=0^{\circ}$ direction by employing parasitic DRA's. One source of x-pol was due to undesired radiating modes that were excited by the hybrid mode of the DIG. Some of these undesired modes can be shorted-out with the addition of a thin metallic rectangle inside the DRA. The frequency dependent phase shift inherent to DRA's was used to counteract the phase progression of a series-fed array. By removing the phase progression in the array the frequency scanning can be reduced. 


\section{Summary of Contribution}

Several contributions were made in this thesis, and are listed below.

1. The DIG fed DRA array was analyzed using CMT. This method proved useful for approximating the power coupling between a DIG and a DRA.

2. CMT theory was used to obtain the first DIG fed DRA arrays with sidelobe control by means of a standard amplitude distribution.

3. Cross-polarized radiation was suppressed by a modifiying a rectangular DRA. The modification was the addition of a metal shorting plate.

4. Parasitic DRA's were used to symmetrically load the original DRA to improve the element pattern when in the vicinity of a DIG.

5. Frequency reduction for a non-resonant series fed array was demonstrated without the use of active components. 


\section{Appendix A}

\section{Mode-Matching Derivation - Two Parallel DIG's}

The modes for two infinitely long DIG's are solved for using a mode-matching formulation. The cross-section of the DIG's were placed in a rectangular waveguide of height $d$ and width $2 p$ to simplify the problem. The effect of these electric walls diminishes as the dimensions $d$ and $p$ increase. Conversely, as these parameters increase the numerical computations become more expensive. For the general case each DIG has a different width, height and permittivity. 


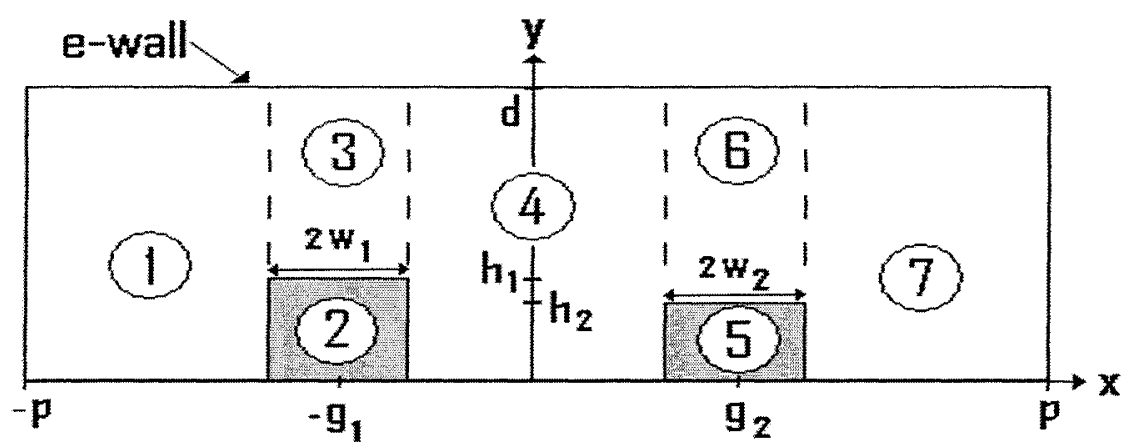

Figure A.1: Cross section of two DIG's in a waveguide

\section{Vector Field Potentials}

The electric and magnetic vector potentials are related to the electric and magnetic scalar potential functions $\left(\phi^{e}\right.$ and $\left.\phi^{h}\right)$ by:

$$
\begin{gathered}
\vec{A}=\hat{y} j \omega \epsilon_{o} \phi^{e} \\
\vec{F}=\hat{y} j \omega \mu_{o} \phi^{h}
\end{gathered}
$$

The perfect electric condutor around the perimeter was enforced by properly selecting the scalar potentials in the regions adjacent to the walls. The summation for each of the scalar potential functions must include an infinite number of terms for an exact answer. To make the numerical computation feasible each of the summations were carried out from the value of $v$ indicated up to $N$. The value of $N$ was the same for all potential functions, and the actual value was determined by studying the convergence of the final solution with respect to $N$. 
The scalar potential functions for each region in Figure A.1 are shown:

$$
\begin{aligned}
\phi_{1}^{e} & =\sum_{v=0} A_{v} \sin \left(\beta_{1 x v}(-x-p)\right) \cos \left(\beta_{1 y v} y\right) \\
\phi_{1}^{h} & =\sum_{v=0} \tilde{A}_{v} \cos \left(\tilde{\beta}_{1 x v}(-x-p)\right) \sin \left(\tilde{\beta}_{1 y v} y\right) \\
\phi_{2}^{e} & =\sum_{v=1}\left[B_{v} \cos \left(\beta_{2 x v}\left(-x-g_{1}\right)\right)+B_{v}^{\prime} \sin \left(\beta_{2 x v}\left(-x-g_{1}\right)\right)\right] \cos \left(\beta_{2 y v} y\right) \\
\phi_{2}^{h} & =\sum_{v=1}\left[\tilde{B}_{v} \sin \left(\tilde{\beta}_{2 x v}\left(-x-g_{1}\right)\right)+\tilde{B}_{v}^{\prime} \cos \left(\tilde{\beta}_{2 x v}\left(-x-g_{1}\right)\right)\right] \sin \left(\tilde{\beta}_{2 y v} y\right) \\
\phi_{3}^{e} & =\sum_{v=1}\left[C_{v} \cos \left(\beta_{3 x v}\left(-x-g_{1}\right)\right)+C_{v}^{\prime} \sin \left(\beta_{3 x v}\left(-x-g_{1}\right)\right)\right] \cos \left(\beta_{3 y v}(y-d)\right) \\
\phi_{3}^{h} & =\sum_{v=1}\left[\tilde{C}_{v} \sin \left(\tilde{\beta}_{3 x v}\left(-x-g_{1}\right)\right)+\tilde{C}_{v}^{\prime} \cos \left(\tilde{\beta}_{3 x v}\left(-x-g_{1}\right)\right)\right] \sin \left(\tilde{\beta}_{3 y v}(y-d)\right) \\
\phi_{4}^{e} & =\sum_{v=0}\left[D_{v} \cos \left(\beta_{4 x v} x\right)+D_{v}^{\prime} \sin \left(\beta_{4 x v} x\right)\right] \cos \left(\beta_{4 y v} y\right) \\
\phi_{4}^{h} & =\sum_{v=0}\left[\tilde{D}_{v} \sin \left(\tilde{\beta}_{4 x v} x\right)+\tilde{D}_{v}{ }^{\prime} \cos \left(\tilde{\beta}_{4 x v} x\right)\right] \sin \left(\tilde{\beta}_{4 y v} y\right) \\
\phi_{5}^{e} & =\sum_{v=1}\left[E_{v} \cos \left(\beta_{5 x v}\left(x-g_{2}\right)\right)+E_{v}^{\prime} \sin \left(\beta_{5 x v}\left(x-g_{2}\right)\right)\right] \cos \left(\beta_{5 y v} y\right) \\
\phi_{5}^{h} & =\sum_{v=1}\left[\tilde{E}_{v} \sin \left(\tilde{\beta}_{5 x v}\left(x-g_{2}\right)\right)+\tilde{E}_{v}^{\prime} \cos \left(\tilde{\beta}_{5 x v}\left(x-g_{2}\right)\right)\right] \sin \left(\tilde{\beta}_{5 y v} y\right) \\
\phi_{7}^{e} & =\sum_{v=1}\left[F_{v} \cos \left(\beta_{6 x v}\left(x-g_{2}\right)\right)+F_{v}^{\prime} \sin \left(\beta_{6 x v}\left(x-g_{2}\right)\right)\right] \cos \left(\beta_{6 y v}(y-d)\right) \\
\phi_{6}^{h} & =\sum_{v=1}^{h}\left[\tilde{F}_{v} \sin \left(\tilde{\beta}_{6 x v}\left(x-g_{2}\right)\right)+\tilde{F}_{v}{ }^{\prime} \cos \left(\tilde{\beta}_{6 x v}\left(x-g_{2}\right)\right)\right] \sin \left(\tilde{\beta}_{6 y v}(y-d)\right) \\
& =\sum_{v=0} G_{v} \sin \left(\tilde{G}_{7 x v} \cos \left(\tilde{\beta}_{7 x v}(x-p)\right) \sin \left(\tilde{\beta}_{7 y v} y\right)\right. \\
&
\end{aligned}
$$

These fields were solved in each of the seven regions. The electric and magnetic field components are calculated from the following equations: 


$$
\begin{aligned}
& \vec{E}=-j \omega \mu_{o} \vec{A}+\frac{1}{j \omega \epsilon} \nabla \nabla \cdot \vec{A}-\nabla \times \vec{F} \\
& \vec{H}=-j \omega \epsilon \vec{F}+\frac{1}{j \omega \mu_{o}} \nabla \nabla \cdot \vec{F}+\nabla \times \vec{A}
\end{aligned}
$$

\section{Region 1}

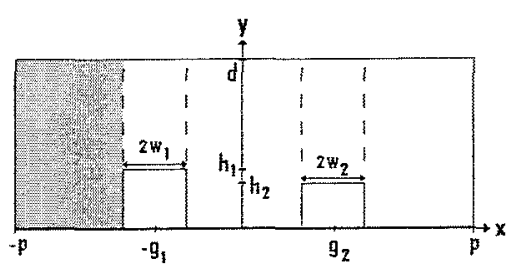

$$
\begin{aligned}
E_{1}^{x} & =\sum_{v=0} \beta_{1 x v} \beta_{1 y v} A_{v} \cos \left(\beta_{1 x v}(-x-p)\right) \sin \left(\beta_{1 y v} y\right) \\
& +\omega \mu_{o} \beta_{z} \sum_{v=0} \tilde{A}_{v} \cos \left(\tilde{\beta}_{1 x v}(-x-p)\right) \sin \left(\tilde{\beta}_{1 y v} y\right) \\
E_{1}^{y} & =\sum_{v=0}\left(k_{o}^{2}-\beta_{1 y v}^{2}\right) A_{v} \sin \left(\beta_{1 x v}(-x-p)\right) \cos \left(\beta_{1 y v} y\right) \\
E_{1}^{z}= & j \beta_{z} \sum_{v=0} \beta_{1 y v} A_{v} \sin \left(\beta_{1 x v}(-x-p)\right) \sin \left(\beta_{1 y v} y\right) \\
& -j \omega \mu_{o} \sum_{v=0} \tilde{\beta}_{1 x v} \tilde{A}_{v} \sin \left(\tilde{\beta}_{1 x v}(-x-p)\right) \sin \left(\tilde{\beta}_{1 y v} y\right) \\
H_{1}^{x} & =-\omega \epsilon_{o} \beta_{z} \sum_{v=0} A_{v} \sin \left(\beta_{1 x v}(-x-p)\right) \cos \left(\beta_{1 y v} y\right) \\
& +\sum_{v=0} \tilde{\beta}_{1 x v} \tilde{\beta}_{1 y v} \tilde{A}_{v} \sin \left(\tilde{\beta}_{1 x v}(-x-p)\right) \cos \left(\tilde{\beta}_{1 y v} y\right) \\
H_{1}^{y} & =\sum_{v=0}\left(k_{o}^{2}-\tilde{\beta}_{1 y v}^{2}\right) \tilde{A}_{v} \cos \left(\tilde{\beta}_{1 x v}(-x-p)\right) \sin \left(\tilde{\beta}_{1 y v} y\right) \\
H_{1}^{z}= & -j \omega \epsilon_{o} \sum_{v=0} \beta_{1 x v} A_{v} \cos \left(\beta_{1 x v}(-x-p)\right) \cos \left(\beta_{1 y v} y\right) \\
& -j \beta_{z} \sum_{v=0} \tilde{\beta}_{1 y v} \tilde{A}_{v} \cos \left(\tilde{\beta}_{1 x v}(-x-p)\right) \cos \left(\tilde{\beta}_{1 y v} y\right)
\end{aligned}
$$




\section{Region 2}

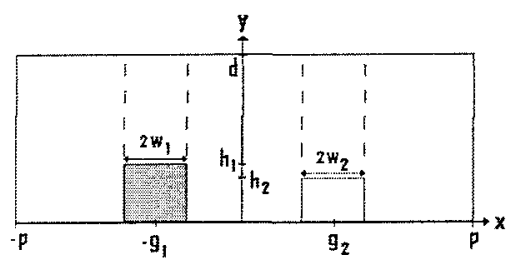

$$
\begin{aligned}
E_{2}^{x} & =-\frac{1}{\epsilon_{r 1}} \sum_{v=1} \beta_{2 x v} \beta_{2 y v}\left[B_{v} \sin \left(\beta_{2 x v}\left(-x-g_{1}\right)\right)-B_{v}^{\prime} \cos \left(\beta_{2 x v}\left(-x-g_{1}\right)\right)\right] \sin \left(\beta_{2 y v} y\right) \\
& +\omega \mu_{o} \beta_{z} \sum_{v=1}\left[\tilde{B}_{v} \sin \left(\tilde{\beta}_{2 x v}\left(-x-g_{1}\right)\right)+\tilde{B}_{v}{ }^{\prime} \cos \left(\tilde{\beta}_{2 x v}\left(-x-g_{1}\right)\right)\right] \sin \left(\tilde{\beta}_{2 y v} y\right) \\
E_{2}^{y}= & \sum_{v=1}\left(k_{o}^{2}-\frac{\beta_{2 y v}^{2}}{\epsilon_{r 1}}\right)\left[B_{v} \cos \left(\beta_{2 x v}\left(-x-g_{1}\right)\right)+B_{v}^{\prime} \sin \left(\beta_{2 x v}\left(-x-g_{1}\right)\right)\right] \cos \left(\beta_{2 y v} y\right) \\
E_{2}^{z}= & j \frac{\beta_{z}}{\epsilon_{r 1}} \sum_{v=1} \beta_{2 y v}\left[B_{v} \cos \left(\beta_{2 x v}\left(-x-g_{1}\right)\right)+B_{v}^{\prime} \sin \left(\beta_{2 x v}\left(-x-g_{1}\right)\right)\right] \sin \left(\beta_{2 y v} y\right) \\
& +j \omega \mu_{o} \sum_{v=1} \tilde{\beta}_{2 x v}\left[\tilde{B}_{v} \cos \left(\tilde{\beta}_{2 x v}\left(-x-g_{1}\right)\right)-\tilde{B}_{v}{ }^{\prime} \sin \left(\tilde{\beta}_{2 x v}\left(-x-g_{1}\right)\right)\right] \sin \left(\tilde{\beta}_{2 y v} y\right) \\
H_{2}^{x}= & -\omega \epsilon_{o} \beta_{z} \sum_{v=1}\left[B_{v} \cos \left(\beta_{2 x v}\left(-x-g_{1}\right)\right)+B_{v}^{\prime} \sin \left(\beta_{2 x v}\left(-x-g_{1}\right)\right)\right] \cos \left(\beta_{2 y v} y\right) \\
& -\sum_{v=1} \tilde{\beta}_{2 x v} \tilde{\beta}_{2 y v}\left[\tilde{B}_{v} \cos \left(\tilde{\beta}_{2 x v}\left(-x-g_{1}\right)\right)-\tilde{B}_{v}{ }^{\prime} \sin \left(\tilde{\beta}_{2 x v}\left(-x-g_{1}\right)\right)\right] \cos \left(\tilde{\beta}_{2 y v} y\right) \\
H_{2}^{y}= & \sum_{v=1}\left(k_{o}^{2} \epsilon_{r 1}-\tilde{\beta}_{2 y v}^{2}\right)\left[\tilde{B}_{v} \sin \left(\tilde{\beta}_{2 x v}\left(-x-g_{1}\right)\right)+\tilde{B}_{v}^{\prime} \cos \left(\tilde{\beta}_{2 x v}\left(-x-g_{1}\right)\right)\right] \sin \left(\tilde{\beta}_{2 y v} y\right) \\
H_{2}^{z}= & j \omega \epsilon_{o} \sum_{v=1} \beta_{2 x v}\left[B_{v} \sin \left(\beta_{2 x v}\left(-x-g_{1}\right)\right)-B_{v}^{\prime} \cos \left(\beta_{2 x v}\left(-x-g_{1}\right)\right)\right] \cos \left(\beta_{2 y v} y\right) \\
& -j \beta_{z} \sum_{v=1} \tilde{\beta}_{2 y v}\left[\tilde{B}_{v} \sin \left(\tilde{\beta}_{2 x v}\left(-x-g_{1}\right)\right)+\tilde{B}_{v}{ }^{\prime} \cos \left(\tilde{\beta}_{2 x v}\left(-x-g_{1}\right)\right)\right] \cos \left(\tilde{\beta}_{2 y v} y\right)
\end{aligned}
$$




\section{Region 3}

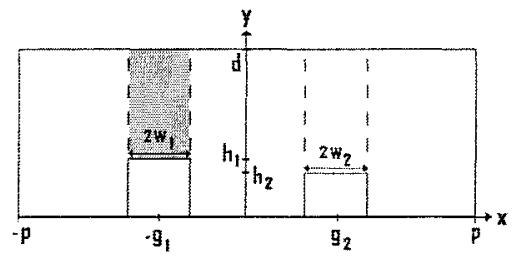

$$
\begin{aligned}
E_{3}^{x} & =-\sum_{v=1} \beta_{3 x v} \beta_{3 y v}\left[C_{v} \sin \left(\beta_{3 x v}\left(-x-g_{1}\right)\right)-C_{v}^{\prime} \cos \left(\beta_{3 x v}\left(-x-g_{1}\right)\right)\right] \sin \left(\beta_{3 y v}(y-d)\right) \\
& +\omega \mu_{o} \beta_{z} \sum_{v=1}\left[\tilde{C}_{v} \sin \left(\tilde{\beta}_{3 x v}\left(-x-g_{1}\right)\right)+\tilde{C}_{v}{ }^{\prime} \cos \left(\tilde{\beta}_{3 x v}\left(-x-g_{1}\right)\right)\right] \sin \left(\tilde{\beta}_{3 y v}(y-d)\right) \\
E_{3}^{y} & =\sum_{v=1}\left(k_{o}^{2}-\beta_{3 y v}^{2}\right)\left[C_{v} \cos \left(\beta_{3 x v}\left(-x-g_{1}\right)\right)+C_{v}^{\prime} \sin \left(\beta_{3 x v}\left(-x-g_{1}\right)\right)\right] \cos \left(\beta_{3 y v}(y-d)\right) \\
E_{3}^{z}= & j \beta_{z} \sum_{v=1} \beta_{3 y v}\left[C_{v} \cos \left(\beta_{3 x v}\left(-x-g_{1}\right)\right)+C_{v}^{\prime} \sin \left(\beta_{3 x v}\left(-x-g_{1}\right)\right)\right] \sin \left(\beta_{3 y v}(y-d)\right) \\
& +j \omega \mu_{o} \sum_{v=1} \tilde{\beta}_{3 x v}\left[\tilde{C}_{v} \cos \left(\tilde{\beta}_{3 x v}\left(-x-g_{1}\right)\right)-\tilde{C}_{v}{ }^{\prime} \sin \left(\tilde{\beta}_{3 x v}\left(-x-g_{1}\right)\right)\right] \sin \left(\tilde{\beta}_{3 y v}(y-d)\right) \\
H_{3}^{x}= & -\omega \epsilon_{o} \beta_{z} \sum_{v=1}\left[C_{v} \cos \left(\beta_{3 x v}\left(-x-g_{1}\right)\right)+C_{v}^{\prime} \sin \left(\beta_{3 x v}\left(-x-g_{1}\right)\right)\right] \cos \left(\beta_{3 y v}(y-d)\right) \\
& -\sum_{v=1} \tilde{\beta}_{3 x v} \tilde{\beta}_{3 y v}\left[\tilde{C}_{v} \cos \left(\tilde{\beta}_{3 x v}\left(-x-g_{1}\right)\right)-\tilde{C}_{v}{ }^{\prime} \sin \left(\tilde{\beta}_{3 x v}\left(-x-g_{1}\right)\right)\right] \cos \left(\tilde{\beta}_{3 y v}(y-d)\right) \\
H_{3}^{y}= & \sum_{v=1}\left(k_{o}^{2}-\tilde{\beta}_{3 y v}^{2}\right)\left[\tilde{C}_{v} \sin \left(\tilde{\beta}_{3 x v}\left(-x-g_{1}\right)\right)+\tilde{C}_{v}^{\prime} \cos \left(\tilde{\beta}_{3 x v}\left(-x-g_{1}\right)\right)\right] \sin \left(\tilde{\beta}_{3 y v}(y-d)\right) \\
H_{3}^{z}= & j \omega \epsilon_{o} \sum_{v=1} \beta_{3 x v}\left[C_{v} \sin \left(\beta_{3 x v}\left(-x-g_{1}\right)\right)-C_{v}^{\prime} \cos \left(\beta_{3 x v}\left(-x-g_{1}\right)\right)\right] \cos \left(\beta_{3 y v}(y-d)\right) \\
& -j \beta_{z} \sum_{v=1} \tilde{\beta}_{3 y v}\left[\tilde{C}_{v} \sin \left(\tilde{\beta}_{3 x v}\left(-x-g_{1}\right)\right)+\tilde{C}_{v}{ }^{\prime} \cos \left(\tilde{\beta}_{3 x v}\left(-x-g_{1}\right)\right)\right] \cos \left(\tilde{\beta}_{3 y v}(y-d)\right)
\end{aligned}
$$




\section{Region 4}

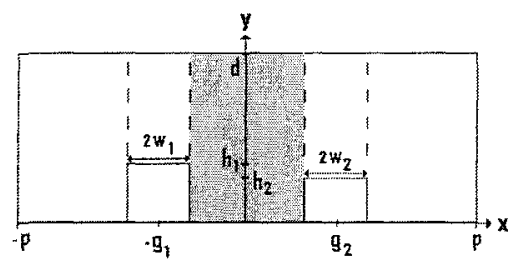

$$
\begin{aligned}
E_{4}^{x} & =\sum_{v=0} \beta_{4 x v} \beta_{4 y v}\left[D_{v} \sin \left(\beta_{4 x v} x\right)-D_{v}^{\prime} \cos \left(\beta_{4 x v} x\right)\right] \sin \left(\beta_{4 y v} y\right) \\
& +\omega \mu_{o} \beta_{z} \sum_{v=0}\left[\tilde{D}_{v} \sin \left(\tilde{\beta}_{4 x v} x\right)+\tilde{D}_{v}^{\prime} \cos \left(\tilde{\beta}_{4 x v} x\right)\right] \sin \left(\tilde{\beta}_{4 y v} y\right) \\
E_{4}^{y}= & \sum_{v=0}\left(k_{o}^{2}-\beta_{4 y v}^{2}\right)\left[D_{v} \cos \left(\beta_{4 x v} x\right)+D_{v}^{\prime} \sin \left(\beta_{4 x v} x\right)\right] \cos \left(\beta_{4 y v} y\right) \\
E_{4}^{z}= & j \beta_{z} \sum_{v=0} \beta_{4 y v}\left[D_{v} \cos \left(\beta_{4 x v} x\right)+D_{v}^{\prime} \sin \left(\beta_{4 x v} x\right)\right] \sin \left(\beta_{4 y v} y\right) \\
& -j \omega \mu_{o} \sum_{v=0} \tilde{\beta}_{4 x v}\left[\tilde{D}_{v} \cos \left(\tilde{\beta}_{4 x v} x\right)-\tilde{D}_{v}^{\prime} \sin \left(\tilde{\beta}_{4 x v} x\right)\right] \sin \left(\tilde{\beta}_{4 y v} y\right) \\
H_{4}^{x}= & -\omega \epsilon_{o} \beta_{z} \sum_{v=0}\left[D_{v} \cos \left(\beta_{4 x v} x\right)+D_{v}^{\prime} \sin \left(\beta_{4 x v} x\right)\right] \cos \left(\beta_{4 y v} y\right) \\
& +\sum_{v=0} \tilde{\beta}_{4 x v} \tilde{\beta}_{4 y v}\left[\tilde{D}_{v} \cos \left(\tilde{\beta}_{4 x v} x\right)-\tilde{D}_{v}^{\prime} \sin \left(\tilde{\beta}_{4 x v} x\right)\right] \cos \left(\tilde{\beta}_{4 y v} y\right) \\
H_{4}^{y}= & \sum_{v=0}\left(k_{o}^{2}-\tilde{\beta}_{4 y v}^{2}\right)\left[\tilde{D}_{v} \sin \left(\tilde{\beta}_{4 x v} x\right)+\tilde{D}_{v}^{\prime} \cos \left(\tilde{\beta}_{4 x v} x\right)\right] \sin \left(\tilde{\beta}_{4 y v} y\right) \\
H_{4}^{z}= & -j \omega \epsilon_{o} \sum_{v=0} \beta_{4 x v}\left[D_{v} \sin \left(\beta_{4 x v} x\right)-D_{v}^{\prime} \cos \left(\beta_{4 x v} x\right)\right] \cos \left(\beta_{4 y v} y\right) \\
& -j \beta_{z} \sum_{v=0} \tilde{\beta}_{4 y v}\left[\tilde{D}_{v} \sin \left(\tilde{\beta}_{4 x v} x\right)+\tilde{D}_{v}^{\prime} \cos \left(\tilde{\beta}_{4 x v} x\right)\right] \cos \left(\tilde{\beta}_{4 y v} y\right)
\end{aligned}
$$




\section{Region 5}

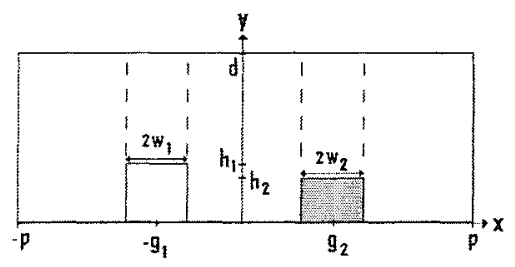

$$
\begin{aligned}
E_{5}^{x} & =\frac{1}{\epsilon_{r 2}} \sum_{v=1} \beta_{5 x v} \beta_{5 y v}\left[E_{v} \sin \left(\beta_{5 x v}\left(x-g_{2}\right)\right)-E_{v}^{\prime} \cos \left(\beta_{5 x v}\left(x-g_{2}\right)\right)\right] \sin \left(\beta_{5 y v} y\right) \\
& +\omega \mu_{o} \beta_{z} \sum_{v=1}\left[\tilde{E}_{v} \sin \left(\tilde{\beta}_{5 x v}\left(x-g_{2}\right)\right)+\tilde{E}_{v}^{\prime} \cos \left(\tilde{\beta}_{5 x v}\left(x-g_{2}\right)\right)\right] \sin \left(\tilde{\beta}_{5 y v} y\right) \\
E_{5}^{y}= & \sum_{v=1}\left(k_{o}^{2}-\frac{\beta_{5 y v}^{2}}{\epsilon_{r 2}}\right)\left[E_{v} \cos \left(\beta_{5 x v}\left(x-g_{2}\right)\right)+E_{v}^{\prime} \sin \left(\beta_{5 x v}\left(x-g_{2}\right)\right)\right] \cos \left(\beta_{5 y v} y\right) \\
E_{5}^{z}= & j \frac{\beta_{z}}{\epsilon_{r 2}} \sum_{v=1} \beta_{5 y v}\left[E_{v} \cos \left(\beta_{5 x v}\left(x-g_{2}\right)\right)+E_{v}^{\prime} \sin \left(\beta_{5 x v}\left(x-g_{2}\right)\right)\right] \sin \left(\beta_{5 y v} y\right) \\
& -j \omega \mu_{o} \sum_{v=1} \tilde{\beta}_{5 x v}\left[\tilde{E}_{v} \cos \left(\tilde{\beta}_{5 x v}\left(x-g_{2}\right)\right)-\tilde{E}_{v}{ }^{\prime} \sin \left(\tilde{\beta}_{5 x v}\left(x-g_{2}\right)\right)\right] \sin \left(\tilde{\beta}_{5 y v} y\right) \\
H_{5}^{x}= & -\omega \epsilon_{o} \beta_{z} \sum_{v=1}\left[E_{v} \cos \left(\beta_{5 x v}\left(x-g_{2}\right)\right)+E_{v}^{\prime} \sin \left(\beta_{5 x v}\left(x-g_{2}\right)\right)\right] \cos \left(\beta_{5 y v} y\right) \\
& +\sum_{v=1} \tilde{\beta}_{5 x v} \tilde{\beta}_{5 y v}\left[\tilde{E}_{v} \cos \left(\tilde{\beta}_{5 x v}\left(x-g_{2}\right)\right)-\tilde{E}_{v}^{\prime} \sin \left(\tilde{\beta}_{5 x v}\left(x-g_{2}\right)\right)\right] \cos \left(\tilde{\beta}_{5 y v} y\right) \\
H_{5}^{y}= & \sum_{v=1}\left(k_{o}^{2} \epsilon_{r 2}-\tilde{\beta}_{5 y v}^{2}\right)\left[\tilde{E}_{v} \sin \left(\tilde{\beta}_{5 x v}\left(x-g_{2}\right)\right)+\tilde{E}_{v}^{\prime} \cos \left(\tilde{\beta}_{5 x v}\left(x-g_{2}\right)\right)\right] \sin \left(\tilde{\beta}_{5 y v} y\right) \\
H_{5}^{z}= & -j \omega \epsilon_{o} \sum_{v=1} \beta_{5 x v}\left[E_{v} \sin \left(\beta_{5 x v}\left(x-g_{2}\right)\right)-E_{v}^{\prime} \cos \left(\beta_{5 x v}\left(x-g_{2}\right)\right)\right] \cos \left(\beta_{5 y v} y\right) \\
& -j \beta_{z} \sum_{v=1} \tilde{\beta}_{5 y v}\left[\tilde{E}_{v} \sin \left(\tilde{\beta}_{5 x v}\left(x-g_{2}\right)\right)+\tilde{E}_{v}{ }^{\prime} \cos \left(\tilde{\beta}_{5 x v}\left(x-g_{2}\right)\right)\right] \cos \left(\tilde{\beta}_{5 y v} y\right)
\end{aligned}
$$




\section{Region 6}

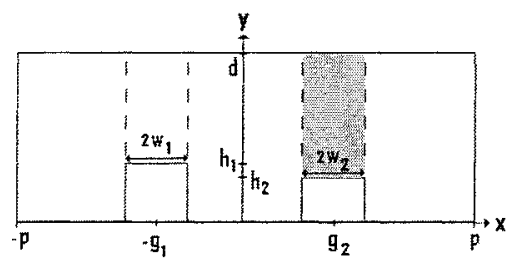

$$
\begin{aligned}
E_{6}^{x} & =\sum_{v=1} \beta_{6 x v} \beta_{6 y v}\left[F_{v} \sin \left(\beta_{6 x v}\left(x-g_{2}\right)\right)-F_{v}^{\prime} \cos \left(\beta_{6 x v}\left(x-g_{2}\right)\right)\right] \sin \left(\beta_{6 y v}(y-d)\right) \\
& +\omega \mu_{o} \beta_{z} \sum_{v=1}\left[\tilde{F}_{v} \sin \left(\tilde{\beta}_{6 x v}\left(x-g_{2}\right)\right)+\tilde{F}_{v}^{\prime} \cos \left(\tilde{\beta}_{6 x v}\left(x-g_{2}\right)\right)\right] \sin \left(\tilde{\beta}_{6 y v}(y-d)\right) \\
E_{6}^{y}= & \sum_{v=1}\left(k_{o}^{2}-\beta_{6 y v}^{2}\right)\left[F_{v} \cos \left(\beta_{6 x v}\left(x-g_{2}\right)\right)+F_{v}^{\prime} \sin \left(\beta_{6 x v}\left(x-g_{2}\right)\right)\right] \cos \left(\beta_{6 y v}(y-d)\right) \\
E_{6}^{z}= & j \beta_{z} \sum_{v=1} \beta_{6 y v}\left[F_{v} \cos \left(\beta_{6 x v}\left(x-g_{2}\right)\right)+F_{v}^{\prime} \sin \left(\beta_{6 x v}\left(x-g_{2}\right)\right)\right] \sin \left(\beta_{6 y v}(y-d)\right) \\
& -j \omega \mu_{o} \sum_{v=1} \tilde{\beta}_{6 x v}\left[\tilde{F}_{v} \cos \left(\tilde{\beta}_{6 x v}\left(x-g_{2}\right)\right)-\tilde{F}_{v}^{\prime} \sin \left(\tilde{\beta}_{6 x v}\left(x-g_{2}\right)\right)\right] \sin \left(\tilde{\beta}_{6 y v}(y-d)\right) \\
H_{6}^{x}= & -\omega \epsilon_{o} \beta_{z} \sum_{v=1}\left[F_{v} \cos \left(\beta_{6 x v}\left(x-g_{2}\right)\right)+F_{v}^{\prime} \sin \left(\beta_{6 x v}\left(x-g_{2}\right)\right)\right] \cos \left(\beta_{6 y v}(y-d)\right) \\
& +\sum_{v=1} \tilde{\beta}_{6 x v} \tilde{\beta}_{6 y v}\left[\tilde{F}_{v} \cos \left(\tilde{\beta}_{6 x v}\left(x-g_{2}\right)\right)-\tilde{F}_{v}^{\prime} \sin \left(\tilde{\beta}_{6 x v}\left(x-g_{2}\right)\right)\right] \cos \left(\tilde{\beta}_{6 y v}(y-d)\right) \\
H_{6}^{y}= & \sum_{v=1}\left(k_{o}^{2}-\tilde{\beta}_{6 y v}^{2}\right)\left[\tilde{F}_{v} \sin \left(\tilde{\beta}_{6 x v}\left(x-g_{2}\right)\right)+\tilde{F}_{v}^{\prime} \cos \left(\tilde{\beta}_{6 x v}\left(x-g_{2}\right)\right)\right] \sin \left(\tilde{\beta}_{6 y v}(y-d)\right) \\
H_{6}^{z}= & -j \omega \epsilon_{o} \sum_{v=1} \beta_{6 x v}\left[F_{v} \sin \left(\beta_{6 x v}\left(x-g_{2}\right)\right)-F_{v}^{\prime} \cos \left(\beta_{6 x v}\left(x-g_{2}\right)\right)\right] \cos \left(\beta_{6 y v}(y-d)\right) \\
& -j \beta_{z} \sum_{v=1} \tilde{\beta}_{6 y v}\left[\tilde{F}_{v} \sin \left(\tilde{\beta}_{6 x v}\left(x-g_{2}\right)\right)+\tilde{F}_{v}^{\prime} \cos \left(\tilde{\beta}_{6 x v}\left(x-g_{2}\right)\right)\right] \cos \left(\tilde{\beta}_{6 y v}(y-d)\right)
\end{aligned}
$$




\section{Region 7}

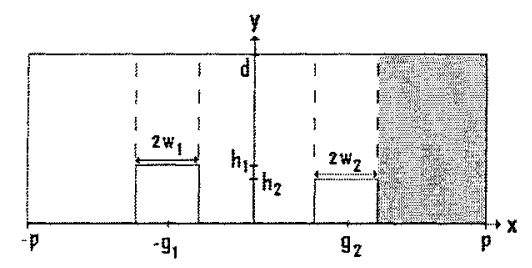

$$
\begin{aligned}
E_{7}^{x} & =-\sum_{v=0} \beta_{7 x v} \beta_{7 y v} G_{v} \cos \left(\beta_{7 x v}(x-p)\right) \sin \left(\beta_{7 y v} y\right) \\
& +\omega \mu_{o} \beta_{z} \sum_{v=0} \tilde{G}_{v} \cos \left(\tilde{\beta}_{7 x v}(x-p)\right) \sin \left(\tilde{\beta}_{7 y v} y\right) \\
E_{7}^{y} & =\sum_{v=0}\left(k_{o}^{2}-\beta_{7 y v}^{2}\right) G_{v} \sin \left(\beta_{7 x v}(x-p)\right) \cos \left(\beta_{7 y v} y\right) \\
E_{7}^{z} & =j \beta_{z} \sum_{v=0} \beta_{7 y v} G_{v} \sin \left(\beta_{7 x v}(x-p)\right) \sin \left(\beta_{7 y v} y\right) \\
& +j \omega \mu_{o} \sum_{v=0} \tilde{\beta}_{7 x v} \tilde{G}_{v} \sin \left(\tilde{\beta}_{7 x v}(x-p)\right) \sin \left(\tilde{\beta}_{7 y v} y\right) \\
H_{7}^{x} & =-\omega \epsilon_{o} \beta_{z} \sum_{v=0} G_{v} \sin \left(\beta_{7 x v}(x-p)\right) \cos \left(\beta_{7 y v} y\right) \\
& -\sum_{v=0} \tilde{\beta}_{7 x v} \tilde{\beta}_{7 y v} \tilde{G}_{v} \sin \left(\tilde{\beta}_{7 x v}(x-p)\right) \cos \left(\tilde{\beta}_{7 y v} y\right) \\
H_{7}^{y} & =\sum_{v=0}\left(k_{o}^{2}-\tilde{\beta}_{7 y v}^{2}\right) \tilde{G}_{v} \cos \left(\tilde{\beta}_{7 x v}(x-p)\right) \sin \left(\tilde{\beta}_{7 y v} y\right) \\
H_{7}^{z} & =j \omega \epsilon_{o} \sum_{v=0} \beta_{7 x v} G_{v} \cos \left(\beta_{7 x v}(x-p)\right) \cos \left(\beta_{7 y v} y\right) \\
& -j \beta_{z} \sum_{v=0} \tilde{\beta}_{7 y v} \tilde{G}_{v} \cos \left(\tilde{\beta}_{7 x v}(x-p)\right) \cos \left(\tilde{\beta}_{7 y v} y\right)
\end{aligned}
$$

In regions 1, 4 and 7 the problem space is bounded above and below by perfect electric conductors. The component of the propagation constants corresponding to the y-dimension is identical to that of a parallel plate waveguide with a spacing $d$ between the plates: 


$$
\beta_{1 y v}=\beta_{4 y v}=\beta_{7 y v}=\tilde{\beta}_{1 y v}=\tilde{\beta_{4 y v}}=\tilde{\beta_{7 y v}}=\frac{v \pi}{d} \text { for } v=0,1,2 \ldots N
$$

The general dispersion equation in region $i$ (for $i=1-7$ ) is given by:

$$
\begin{aligned}
& \beta_{i y v}^{2}+\beta_{i x v}^{2}+\beta_{z}^{2}=k_{o}^{2} \epsilon_{r i} \\
& \tilde{\beta}_{i y v}^{2}+\tilde{\beta}_{i x v}^{2}+\beta_{z}^{2}=k_{o}^{2} \epsilon_{r i}
\end{aligned}
$$

Two functions that are used throughout the remainder of the derivation are shown below with their definitions.

$$
\begin{aligned}
& \delta_{c}(n)=\delta_{c}= \begin{cases}2, & n=0 \\
1, & n \neq 0\end{cases} \\
& \delta_{s}(n)=\delta_{s}= \begin{cases}0, & n=0 \\
1, & n \neq 0\end{cases}
\end{aligned}
$$

In the proceeding derivation the terms $I$ and $\tilde{I}$ are used. The closed form expressions for these terms are shown at the end of the derivation. 
The boundary conditions at $y=h_{1}$ were enforced for $-g_{1}-w_{1}<x<$ $-g_{1}+w_{1}$

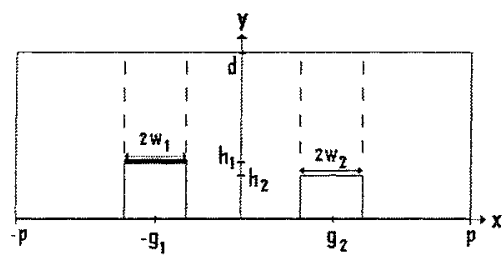

a) Match $E^{z}$ at $y=h_{1}$ for $-g_{1}-w_{1}<x<-g_{1}+w_{1}$ :

$$
\begin{aligned}
E_{2}^{z} & =E_{3}^{z} \\
& j \frac{\beta_{z}}{\epsilon_{r 1}} \sum_{v=1} \beta_{2 y v}\left[B_{v} \cos \left(\beta_{2 x v}\left(-x-g_{1}\right)\right)+B_{v}^{\prime} \sin \left(\beta_{2 x v}\left(-x-g_{1}\right)\right)\right] \sin \left(\beta_{2 y v} h_{1}\right) \\
& +j \omega \mu_{o} \sum_{v=1} \tilde{\beta}_{2 x v}\left[\tilde{B}_{v} \cos \left(\tilde{\beta}_{2 x v}\left(-x-g_{1}\right)\right)-\tilde{B}_{v}{ }^{\prime} \sin \left(\tilde{\beta}_{2 x v}\left(-x-g_{1}\right)\right)\right] \sin \left(\tilde{\beta}_{2 y v} h_{1}\right) \\
& =j \beta_{z} \sum_{v=1} \beta_{3 y v}\left[C_{v} \cos \left(\beta_{3 x v}\left(-x-g_{1}\right)\right)+C_{v}^{\prime} \sin \left(\beta_{3 x v}\left(-x-g_{1}\right)\right)\right] \sin \left(\beta_{3 y v}\left(h_{1}-d\right)\right) \\
& +j \omega \mu_{o} \sum_{v=1} \tilde{\beta}_{3 x v}\left[\tilde{C}_{v} \cos \left(\tilde{\beta}_{3 x v}\left(-x-g_{1}\right)\right)-\tilde{C}_{v}{ }^{\prime} \sin \left(\tilde{\beta}_{3 x v}\left(-x-g_{1}\right)\right)\right] \sin \left(\tilde{\beta}_{3 y v}\left(h_{1}-d\right)\right)
\end{aligned}
$$


b) Match $H^{z}$ at $y=h_{1}$ for $-g_{1}-w_{1}<x<-g_{1}+w_{1}$ :

$$
\begin{aligned}
H_{2}^{z} & =H_{3}^{z} \\
& j \omega \epsilon_{o} \sum_{v=1} \beta_{2 x v}\left[B_{v} \sin \left(\beta_{2 x v}\left(-x-g_{1}\right)\right)-B_{v}^{\prime} \cos \left(\beta_{2 x v}\left(-x-g_{1}\right)\right)\right] \cos \left(\beta_{2 y v} h_{1}\right) \\
& -j \beta_{z} \sum_{v=1} \tilde{\beta}_{2 y v}\left[\tilde{B}_{v} \sin \left(\tilde{\beta}_{2 x v}\left(-x-g_{1}\right)\right)+\tilde{B}_{v}^{\prime} \cos \left(\tilde{\beta}_{2 x v}\left(-x-g_{1}\right)\right)\right] \cos \left(\tilde{\beta}_{2 y v} h_{1}\right) \\
& =j \omega \epsilon_{o} \sum_{v=1} \beta_{3 x v}\left[C_{v} \sin \left(\beta_{3 x v}\left(-x-g_{1}\right)\right)-C_{v}^{\prime} \cos \left(\beta_{3 x v}\left(-x-g_{1}\right)\right)\right] \cos \left(\beta_{3 y v}\left(h_{1}-d\right)\right) \\
& -j \beta_{z} \sum_{v=1} \tilde{\beta}_{3 y v}\left[\tilde{C}_{v} \sin \left(\tilde{\beta}_{3 x v}\left(-x-g_{1}\right)\right)+\tilde{C}_{v}^{\prime} \cos \left(\tilde{\beta}_{3 x v}\left(-x-g_{1}\right)\right)\right] \cos \left(\tilde{\beta}_{3 y v}\left(h_{1}-d\right)\right)
\end{aligned}
$$

c) Match $E^{x}$ at $y=h_{1}$ for $-g_{1}-w_{1}<x<-g_{1}+w_{1}$ :

$$
\begin{aligned}
E_{2}^{x} & =E_{3}^{x} \\
& -\frac{1}{\epsilon_{r 1}} \sum_{v=1} \beta_{2 x v} \beta_{2 y v}\left[B_{v} \sin \left(\beta_{2 x v}\left(-x-g_{1}\right)\right)-B_{v}^{\prime} \cos \left(\beta_{2 x v}\left(-x-g_{1}\right)\right)\right] \sin \left(\beta_{2 y v} h_{1}\right) \\
& +\omega \mu_{o} \beta_{z} \sum_{v=1}\left[\tilde{B}_{v} \sin \left(\tilde{\beta}_{2 x v}\left(-x-g_{1}\right)\right)+\tilde{B}_{v}{ }^{\prime} \cos \left(\tilde{\beta}_{2 x v}\left(-x-g_{1}\right)\right)\right] \sin \left(\tilde{\beta}_{2 y v} h_{1}\right) \\
& =\sum_{v=1} \beta_{3 x v} \beta_{3 y v}\left[C_{v} \sin \left(\beta_{3 x v}\left(x+g_{1}\right)\right)+C_{v}^{\prime} \cos \left(\beta_{3 x v}\left(x+g_{1}\right)\right)\right] \sin \left(\beta_{3 y v}\left(h_{1}-d\right)\right) \\
& +\omega \mu_{o} \beta_{z} \sum_{v=1}\left[\tilde{C}_{v} \sin \left(\tilde{\beta}_{3 x v}\left(-x-g_{1}\right)\right)+\tilde{C}_{v}{ }^{\prime} \cos \left(\tilde{\beta}_{3 x v}\left(-x-g_{1}\right)\right)\right] \sin \left(\tilde{\beta}_{3 y v}\left(h_{1}-d\right)\right)
\end{aligned}
$$


d) Match $H^{x}$ at $y=h_{1}$ for $-g_{1}-w_{1}<x<-g_{1}+w_{1}$ :

$$
\begin{aligned}
& -H_{2}^{x}=-H_{3}^{x} \\
& \quad \omega \epsilon_{o} \beta_{z} \sum_{v=1}\left[B_{v} \cos \left(\beta_{2 x v}\left(-x-g_{1}\right)\right)+B_{v}^{\prime} \sin \left(\beta_{2 x v}\left(-x-g_{1}\right)\right)\right] \cos \left(\beta_{2 y v} h_{1}\right) \\
& \quad+\sum_{v=1} \tilde{\beta}_{2 x v} \tilde{\beta}_{2 y v}\left[\tilde{B}_{v} \cos \left(\tilde{\beta}_{2 x v}\left(-x-g_{1}\right)\right)-\tilde{B}_{v}^{\prime} \sin \left(\tilde{\beta}_{2 x v}\left(-x-g_{1}\right)\right)\right] \cos \left(\tilde{\beta}_{2 y v} h_{1}\right) \\
& =\omega \epsilon_{o} \beta_{z} \sum_{v=1}\left[C_{v} \cos \left(\beta_{3 x v}\left(-x-g_{1}\right)\right)+C_{v}^{\prime} \sin \left(\beta_{3 x v}\left(-x-g_{1}\right)\right)\right] \cos \left(\beta_{3 y v}\left(h_{1}-d\right)\right) \\
& \quad+\sum_{v=1} \tilde{\beta}_{3 x v} \tilde{\beta}_{3 y v}\left[\tilde{C}_{v} \cos \left(\tilde{\beta}_{3 x v}\left(-x-g_{1}\right)\right)-\tilde{C}_{v}{ }^{\prime} \sin \left(\tilde{\beta}_{3 x v}\left(-x-g_{1}\right)\right)\right] \cos \left(\tilde{\beta}_{3 y v}\left(h_{1}-d\right)\right)
\end{aligned}
$$

e) Match $D^{y}$ at $y=h_{1}$ for $-g_{1}-w_{1}<x<-g_{1}+w_{1}$ :

$$
\begin{aligned}
& \epsilon_{r 1} E_{2}^{y}=E_{3}^{y} \\
& \quad \epsilon_{r 1} \sum_{v=1}\left(k_{o}^{2}-\frac{\beta_{2 y v}^{2}}{\epsilon_{r 1}}\right)\left[B_{v} \cos \left(\beta_{2 x v}\left(-x-g_{1}\right)\right)+B_{v}^{\prime} \sin \left(\beta_{2 x v}\left(-x-g_{1}\right)\right)\right] \cos \left(\beta_{2 y v} h_{1}\right) \\
& \quad=\sum_{v=1}\left(k_{o}^{2}-\beta_{3 y v}^{2}\right)\left[C_{v} \cos \left(\beta_{3 x v}\left(x+g_{1}\right)\right)-C_{v}^{\prime} \sin \left(\beta_{3 x v}\left(x+g_{1}\right)\right)\right] \cos \left(\beta_{3 y v}\left(h_{1}-d\right)\right)
\end{aligned}
$$

f) Match $B^{y}$ at $y=h_{1}$ for $-g_{1}-w_{1}<x<-g_{1}+w_{1}$ :

$$
\begin{aligned}
H_{2}^{y} & =H_{3}^{y} \\
& \sum_{v=1}\left(k_{o}^{2} \epsilon_{r 1}-\tilde{\beta}_{2 y v}^{2}\right)\left[\tilde{B}_{v} \sin \left(\tilde{\beta}_{2 x v}\left(-x-g_{1}\right)\right)+\tilde{B}_{v}^{\prime} \cos \left(\tilde{\beta}_{2 x v}\left(-x-g_{1}\right)\right)\right] \sin \left(\tilde{\beta}_{2 y v} h_{1}\right) \\
& =\sum_{v=1}\left(k_{o}^{2}-\tilde{\beta}_{3 y v}^{2}\right)\left[-\tilde{C}_{v} \sin \left(\tilde{\beta}_{3 x v}\left(x+g_{1}\right)\right)+\tilde{C}_{v}^{\prime} \cos \left(\tilde{\beta}_{3 x v}\left(x+g_{1}\right)\right)\right] \sin \left(\tilde{\beta}_{3 y v}\left(h_{1}-d\right)\right)
\end{aligned}
$$


Manipulation of the boundary conditions at $y=h_{1}$ for $-g_{1}-w_{1}<x<$ $-g_{1}+w_{1}$

i) Summing conditions b) and c):

$$
\begin{aligned}
& \sum_{v=1} \beta_{2 x v}\left[B_{v} \sin \left(\beta_{2 x v}\left(-x-g_{1}\right)\right)-B_{v}^{\prime} \cos \left(\beta_{2 x v}\left(-x-g_{1}\right)\right)\right] \\
& \times\left(j \omega \epsilon_{o} \cos \left(\beta_{2 y v} h_{1}\right)-\frac{\beta_{2 y v}}{\epsilon_{r 1}} \sin \left(\beta_{2 y v} h_{1}\right)\right) \\
&+\beta_{z} \sum_{v=1}\left[\tilde{B}_{v} \sin \left(\tilde{\beta}_{2 x v}\left(-x-g_{1}\right)\right)+\tilde{B}_{v}^{\prime} \cos \left(\tilde{\beta}_{2 x v}\left(-x-g_{1}\right)\right)\right] \\
& \times\left(-j \tilde{\beta}_{2 y v} \cos \left(\tilde{\beta}_{2 y v} h_{1}\right)+\omega \mu_{o} \sin \left(\tilde{\beta}_{2 y v} h_{1}\right)\right) \\
&=\sum_{v=1} \beta_{3 x v}\left[C_{v} \sin \left(\beta_{3 x v}\left(-x-g_{1}\right)\right)-C_{v}^{\prime} \cos \left(\beta_{3 x v}\left(-x-g_{1}\right)\right)\right] \\
& \quad \times\left(j \omega \epsilon_{o} \cos \left(\beta_{3 y v}\left(h_{1}-d\right)\right)-\beta_{3 y v} \sin \left(\beta_{3 y v}\left(h_{1}-d\right)\right)\right) \\
& \quad+\beta_{z} \sum_{v=1}\left[\tilde{C}_{v} \sin \left(\tilde{\beta}_{3 x v}\left(-x-g_{1}\right)\right)+\tilde{C}_{v}{ }^{\prime} \cos \left(\tilde{\beta}_{3 x v}\left(-x-g_{1}\right)\right)\right] \\
& \quad \times\left(-j \tilde{\beta}_{3 y v} \cos \left(\tilde{\beta}_{3 y v}\left(h_{1}-d\right)\right)+\omega \mu_{o} \sin \left(\tilde{\beta}_{3 y v}\left(h_{1}-d\right)\right)\right)
\end{aligned}
$$


ii) Summing equations a) and d):

$$
\begin{aligned}
\beta_{z} \sum_{v=1}\left[B_{v} \cos \left(\beta_{2 x v}\left(-x-g_{1}\right)\right)+B_{v}^{\prime} \sin \left(\beta_{2 x v}\left(-x-g_{1}\right)\right)\right] \\
\quad \times\left(j \frac{\beta_{2 y v}}{\epsilon_{r 1}} \sin \left(\beta_{2 y v} h_{1}\right)+\omega \epsilon_{o} \cos \left(\beta_{2 y v} h_{1}\right)\right) \\
\quad+\sum_{v=1} \tilde{\beta}_{2 x v}\left[\tilde{B}_{v} \cos \left(\tilde{\beta}_{2 x v}\left(-x-g_{1}\right)\right)-\tilde{B}_{v}^{\prime} \sin \left(\tilde{\beta}_{2 x v}\left(-x-g_{1}\right)\right)\right] \\
\quad \times\left(j \omega \mu_{o} \sin \left(\tilde{\beta}_{2 y v} h_{1}\right)+\tilde{\beta}_{2 y v} \cos \left(\tilde{\beta}_{2 y v} h_{1}\right)\right) \\
=\beta_{z} \sum_{v=1}\left[C_{v} \cos \left(\beta_{3 x v}\left(-x-g_{1}\right)\right)+C_{v}^{\prime} \sin \left(\beta_{3 x v}\left(-x-g_{1}\right)\right)\right] \\
\quad \times\left(j \beta_{3 y v} \sin \left(\beta_{3 y v}\left(h_{1}-d\right)\right)+\omega \epsilon_{o} \cos \left(\beta_{3 y v}\left(h_{1}-d\right)\right)\right) \\
\quad+\sum_{v=1} \tilde{\beta}_{3 x v}\left[\tilde{C}_{v} \cos \left(\tilde{\beta}_{3 x v}\left(-x-g_{1}\right)\right)-\tilde{C}_{v}{ }^{\prime} \sin \left(\tilde{\beta}_{3 x v}\left(-x-g_{1}\right)\right)\right] \\
\quad \times\left(j \omega \mu_{o} \sin \left(\tilde{\beta}_{3 y v}\left(h_{1}-d\right)\right)+\tilde{\beta}_{3 y v} \cos \left(\tilde{\beta}_{3 y v}\left(h_{1}-d\right)\right)\right)
\end{aligned}
$$

ii-mod) The summation of a) and d) can be manipulated the following form:

$$
\begin{aligned}
-\beta_{z} & \sum_{v=1}\left[B_{v} \cos \left(\beta_{2 x v}\left(-x-g_{1}\right)\right)+B_{v}^{\prime} \sin \left(\beta_{2 x v}\left(-x-g_{1}\right)\right)\right] \\
& \times\left(j \omega \epsilon_{o} \cos \left(\beta_{2 y v} h_{1}\right)-\frac{\beta_{2 y v}}{\epsilon_{r 1}} \sin \left(\beta_{2 y v} h_{1}\right)\right) \\
& +\sum_{v=1} \tilde{\beta}_{2 x v}\left[\tilde{B}_{v} \cos \left(\tilde{\beta}_{2 x v}\left(-x-g_{1}\right)\right)-\tilde{B}_{v}^{\prime} \sin \left(\tilde{\beta}_{2 x v}\left(-x-g_{1}\right)\right)\right] \\
& \times\left(-j \tilde{\beta}_{2 y v} \cos \left(\tilde{\beta}_{2 y v} h_{1}\right)+\omega \mu_{o} \sin \left(\tilde{\beta}_{2 y v} h_{1}\right)\right) \\
=- & \beta_{z} \sum_{v=1}\left[C_{v} \cos \left(\beta_{3 x v}\left(-x-g_{1}\right)\right)+C_{v}^{\prime} \sin \left(\beta_{3 x v}\left(-x-g_{1}\right)\right)\right] \\
& \times\left(j \omega \epsilon_{o} \cos \left(\beta_{3 y v}\left(h_{1}-d\right)\right)-\beta_{3 y v} \sin \left(\beta_{3 y v}\left(h_{1}-d\right)\right)\right) \\
& +\sum_{v=1} \tilde{\beta}_{3 x v}\left[\tilde{C}_{v} \cos \left(\tilde{\beta}_{3 x v}\left(-x-g_{1}\right)\right)-\tilde{C}_{v}^{\prime} \sin \left(\tilde{\beta}_{3 x v}\left(-x-g_{1}\right)\right)\right] \\
& \times\left(-j \tilde{\beta}_{3 y v} \cos \left(\tilde{\beta}_{3 y v}\left(h_{1}-d\right)\right)+\omega \mu_{o} \sin \left(\tilde{\beta}_{3 y v}\left(h_{1}-d\right)\right)\right)
\end{aligned}
$$


The equations from i) and ii-mod) can be each rearranged to equal to zero (ie. LHS-RHS=0). These resulting equations can then be set equal to each other. This result is shown in vector form below, where the terms that are a function of $\mathrm{x}$ in i) have been renamed $f(x)$ and $g(x)$ in ii-mod), and those not a function of $\mathrm{x}$ are represented with $h$ terms.

$$
\left[h_{1 v} h_{2 v} h_{3 v} h_{4 v}\right] \cdot\left[\begin{array}{c}
f_{1 v}(x) \\
f_{2 v}(x) \\
f_{3 v}(x) \\
f_{4 v}(x)
\end{array}\right]=\left[\begin{array}{lll}
h_{1 v} h_{2 v} h_{3 v} h_{4 v}
\end{array}\right] \cdot\left[\begin{array}{l}
g_{1 v}(x) \\
g_{2 v}(x) \\
g_{3 v}(x) \\
g_{4 v}(x)
\end{array}\right]
$$

Since these equations must hold for all x over edge, $f_{1 v}(x)=g_{1 v}(x), f_{2 v}(x)=$ $g_{2 v}(x), f_{3 v}(x)=g_{3 v}(x)$, and $f_{4 v}(x)=g_{4 v}(x)$. From these results it can be shown that:

$$
\begin{aligned}
& \beta_{2 x v}\left[B_{v} \sin \left(\beta_{2 x v}\left(-x-g_{1}\right)\right)-B_{v}^{\prime} \cos \left(\beta_{2 x v}\left(-x-g_{1}\right)\right)\right] \\
& =-\beta_{z}\left[B_{v} \cos \left(\beta_{2 x v}\left(-x-g_{1}\right)\right)+B_{v}^{\prime} \sin \left(\beta_{2 x v}\left(-x-g_{1}\right)\right)\right] \\
& \beta_{z}\left[\tilde{B}_{v} \sin \left(\tilde{\beta}_{2 x v}\left(-x-g_{1}\right)\right)+\tilde{B}_{v}^{\prime} \cos \left(\tilde{\beta}_{2 x v}\left(-x-g_{1}\right)\right)\right] \\
& =\tilde{\beta}_{2 x v}\left[\tilde{B}_{v} \cos \left(\tilde{\beta}_{2 x v}\left(-x-g_{1}\right)\right)-\tilde{B}_{v}{ }^{\prime} \sin \left(\tilde{\beta}_{2 x v}\left(-x-g_{1}\right)\right)\right] \\
& \beta_{3 x v}\left[C_{v} \sin \left(\beta_{3 x v}\left(-x-g_{1}\right)\right)-C_{v}^{\prime} \cos \left(\beta_{3 x v}\left(-x-g_{1}\right)\right)\right] \\
& =-\beta_{z}\left[C_{v} \cos \left(\beta_{3 x v}\left(-x-g_{1}\right)\right)+C_{v}^{\prime} \sin \left(\beta_{3 x v}\left(-x-g_{1}\right)\right)\right] \\
& \beta_{z}\left[\tilde{C}_{v} \sin \left(\tilde{\beta}_{3 x v}\left(-x-g_{1}\right)\right)+\tilde{C}_{v}{ }^{\prime} \cos \left(\tilde{\beta}_{3 x v}\left(-x-g_{1}\right)\right)\right] \\
& =\tilde{\beta}_{3 x v}\left[\tilde{C}_{v} \cos \left(\tilde{\beta}_{3 x v}\left(-x-g_{1}\right)\right)-\tilde{C}_{v}{ }^{\prime} \sin \left(\tilde{\beta}_{3 x v}\left(-x-g_{1}\right)\right)\right]
\end{aligned}
$$


From these equations, after applying some triginometric identities the following equations result:

$$
\begin{aligned}
& \frac{1}{\beta_{z}}=\frac{1}{\beta_{2 x v}} \tan \left(\beta_{2 x v}\left(-x-g_{1}\right)+\tan ^{-1} \frac{B_{v}}{B_{v}^{\prime}}\right) \\
& \frac{1}{\beta_{z}}=\frac{1}{\tilde{\beta}_{2 x v}} \tan \left(\tilde{\beta}_{2 x v}\left(-x-g_{1}\right)+\tan ^{-1} \frac{\tilde{B}_{v}}{\tilde{B}_{v}^{\prime}}\right) \\
& \frac{1}{\beta_{z}}=\frac{1}{\beta_{3 x v}} \tan \left(\beta_{3 x v}\left(-x-g_{1}\right)+\tan ^{-1} \frac{C_{v}}{C_{v}^{\prime}}\right) \\
& \frac{1}{\beta_{z}}=\frac{1}{\tilde{\beta}_{3 x v}} \tan \left(\tilde{\beta}_{3 x v}\left(-x-g_{1}\right)+\tan ^{-1} \frac{\tilde{C}_{v}}{\tilde{C}_{v}^{\prime}}\right)
\end{aligned}
$$

Combining these equations:

$$
\begin{aligned}
& \frac{1}{\beta_{2 x v}} \tan \left(\beta_{2 x v}\left(-x-g_{1}\right)+\tan ^{-1} \frac{B_{v}}{B_{v}^{\prime}}\right)=\frac{1}{\beta_{3 x v}} \tan \left(\beta_{3 x v}\left(-x-g_{1}\right)+\tan ^{-1} \frac{C_{v}}{C_{v}^{\prime}}\right) \\
& \frac{1}{\tilde{\beta}_{2 x v}} \tan \left(\tilde{\beta}_{2 x v}\left(-x-g_{1}\right)+\tan ^{-1} \frac{\tilde{B}_{v}}{\tilde{B}_{v}^{\prime}}\right)=\frac{1}{\tilde{\beta}_{3 x v}} \tan \left(\tilde{\beta}_{3 x v}\left(-x-g_{1}\right)+\tan ^{-1} \frac{\tilde{C}_{v}}{\tilde{C}_{v}^{\prime}}\right)
\end{aligned}
$$

The argument of the tangent function that makes the expression zero is $m \pi$ $(|m|=0,1,2 \ldots)$. With this in mind, the only situation when this equation is satisfied is when the argument of the tan on the LHS is equal to the argument of the $\tan$ on the RHS plus some additional $m \pi$ term:

$$
\begin{aligned}
& \beta_{2 x v}\left(-x-g_{1}\right)+\tan ^{-1} \frac{B_{v}}{B_{v}^{\prime}}=\beta_{3 x v}\left(-x-g_{1}\right)+\tan ^{-1} \frac{C_{v}}{C_{v}^{\prime}}+m \pi \\
& \tilde{\beta}_{2 x v}\left(-x-g_{1}\right)+\tan ^{-1} \frac{\tilde{B}_{v}}{\tilde{B}_{v}^{\prime}}=\tilde{\beta}_{3 x v}\left(-x-g_{1}\right)+\tan ^{-1} \frac{\tilde{C}_{v}}{\tilde{C}_{v}^{\prime}}+m \pi
\end{aligned}
$$


From these results, $\mathrm{m}$ is assumed to be zero, therefore $\beta_{2 x v}=\beta_{3 x v}$ and $\tilde{\beta}_{2 x v}=$ $\tilde{\beta}_{3 x v}$. Furthermore, the ratio $\frac{B_{v}}{B_{v}^{\prime}}=\frac{C_{v}}{C_{v}^{\prime}}$, and since $B_{v}$ and $B_{v}^{\prime}$ are independent it is concluded that $C_{v}=T_{C B} B_{v}$ and $C_{v}^{\prime}=T_{C B} B_{v}^{\prime}$. Similarly for the other two expressions, $\tilde{C}_{v}=\tilde{T}_{C B} \tilde{B}_{v}$, and $\tilde{C}_{v}^{\prime}=\tilde{T}_{C B} \tilde{B}_{v}^{\prime}$. The factors $T_{C B}$ and $\tilde{T}_{C B}$ are to be determined.

From the remaining boundary conditions, the $T$ factors are calculated.

$$
\begin{array}{r}
T_{C B}=\frac{\cos \left(\beta_{2 y v} h_{1}\right)}{\epsilon_{r 1} \cos \left(\beta_{3 y v}\left(h_{1}-d\right)\right)} \\
\tilde{T}_{C B}=\frac{\sin \left(\tilde{\beta}_{2 y v} h_{1}\right)}{\sin \left(\tilde{\beta}_{3 y v}\left(h_{1}-d\right)\right)}
\end{array}
$$

Similarly the boundary conditions at $y=h_{2}$ can be enforced for $g_{2}-w_{2} \leq x \leq$ $g_{2}+w_{2}$ to obtain a simlar result. The resulting $T$ factors are shown below.

$$
\begin{array}{r}
T_{F E}=\frac{\cos \left(\beta_{5 y v} h_{2}\right)}{\epsilon_{r 2} \cos \left(\beta_{6 y v}\left(h_{2}-d\right)\right)} \\
\tilde{T}_{F E}=\frac{\sin \left(\tilde{\beta}_{5 y v} h_{2}\right)}{\sin \left(\tilde{\beta}_{6 y v}\left(h_{2}-d\right)\right)}
\end{array}
$$


The boundary conditions at $x=-g_{1}-w_{1}$ were enforced:

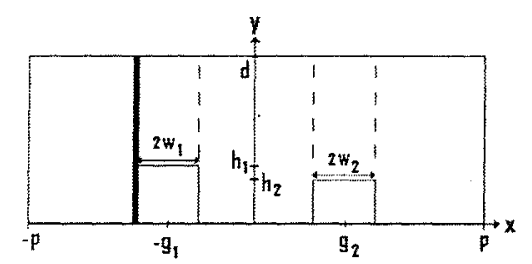

a) Match $E_{y}$ at $x=-g_{1}-w_{1}$

$$
\begin{gathered}
\int_{0}^{d} E_{1}^{y} \cos \frac{n \pi y}{d} d y=\int_{0}^{h_{1}} E_{2}^{y} \cos \frac{n \pi y}{d} d y+\int_{h_{1}}^{d} E_{3}^{y} \cos \frac{n \pi y}{d} d y \text { for } \mathrm{n}=0,1 \ldots \mathrm{N}-1 \\
\delta_{c} \frac{d}{2}\left(k_{o}^{2}-\beta_{1 y n}^{2}\right) A_{n} \sin \left(\beta_{1 x n}\left(g_{1}+w_{1}-p\right)\right) \\
\quad=\sum_{v=1}\left(\beta_{z}^{2}+\beta_{2 x v}^{2}\right)\left[B_{v} \cos \left(\beta_{2 x v} w_{1}\right)+B_{v}^{\prime} \sin \left(\beta_{2 x v} w_{1}\right)\right]\left(\frac{I_{1}^{\beta_{2}}}{\epsilon_{r 1}}+T_{C B} I_{2}^{\beta_{3}}\right)
\end{gathered}
$$

b) Match $H_{y}$ at $x=-g_{1}-w_{1}$

$$
\begin{gathered}
\int_{0}^{d} H_{1}^{y} \sin \frac{n \pi y}{d} d y=\int_{0}^{h_{1}} H_{2}^{y} \sin \frac{n \pi y}{d} d y+\int_{h_{1}}^{d} H_{3}^{y} \sin \frac{n \pi y}{d} d y \text { for } \mathrm{n}=1,2 \ldots \mathrm{N}-1 \\
\delta_{s} \frac{d}{2}\left(k_{o}^{2}-\tilde{\beta}_{1 y n}^{2}\right) \tilde{A}_{n} \cos \left(\tilde{\beta}_{1 x n}\left(g_{1}+w_{1}-p\right)\right) \\
=\sum_{v=1}\left(\beta_{z}^{2}+\tilde{\beta}_{2 x v}^{2}\right)\left(\tilde{B}_{v} \sin \left(\tilde{\beta}_{2 x v} w_{1}\right)+\tilde{B}_{v}^{\prime} \cos \left(\tilde{\beta}_{2 x v} w_{1}\right)\right)\left(\tilde{I}_{3}^{\beta_{2}}+\tilde{T}_{C B} \tilde{I}_{4}^{\beta_{3}}\right)
\end{gathered}
$$


c) Match $E_{z}$ at $x=-g_{1}-w_{1}$

$$
\begin{aligned}
& \int_{0}^{d} E_{1}^{z} \sin \frac{n \pi y}{d} d y=\int_{0}^{h_{1}} E_{2}^{z} \sin \frac{n \pi y}{d} d y+\int_{h_{1}}^{d} E_{3}^{z} \sin \frac{n \pi y}{d} d y \text { for } \mathrm{n}=1,2 \ldots \mathrm{N}-1 \\
& \\
& j \delta_{s} \frac{d}{2}\left[\beta_{z} \beta_{1 y n} A_{n} \sin \left(\beta_{1 x n}\left(g_{1}+w_{1}-p\right)\right)-\omega \mu_{o} \tilde{\beta}_{1 x n} \tilde{A}_{n} \sin \left(\tilde{\beta}_{1 x n}\left(g_{1}+w_{1}-p\right)\right)\right] \\
& \quad=j \beta_{z} \sum_{v=1}\left[B_{v} \cos \left(\beta_{2 x v} w_{1}\right)+B_{v}^{\prime} \sin \left(\beta_{2 x v} w_{1}\right)\right]\left(\frac{\beta_{2 y v}}{\epsilon_{r 1}} I_{3}^{\beta_{2}}+\beta_{3 y v} T_{C B} I_{4}^{\beta_{3}}\right) \\
& \quad+j \omega \mu_{o} \sum_{v=1} \tilde{\beta}_{2 x v}\left[\tilde{B}_{v} \cos \left(\tilde{\beta}_{2 x v} w_{1}\right)-\tilde{B}_{v}{ }^{\prime} \sin \left(\tilde{\beta}_{2 x v} w_{1}\right)\right]\left(\tilde{I}_{3}+\tilde{T}_{C B} \tilde{I}_{4}\right)
\end{aligned}
$$

d) Match $H_{z}$ at $x=-g_{1}-w_{1}$

$$
\begin{aligned}
& \int_{0}^{d} H_{1}^{z} \cos \frac{n \pi y}{d} d y=\int_{0}^{h_{1}} H_{2}^{z} \cos \frac{n \pi y}{d} d y+\int_{h_{1}}^{d} H_{3}^{z} \cos \frac{n \pi y}{d} d y \text { for } \mathrm{n}=0,1 \ldots \mathrm{N}-1 \\
& -j \delta_{c} \frac{d}{2}\left[\omega \epsilon_{o} \beta_{1 x n} A_{n} \cos \left(\beta_{1 x n}\left(g_{1}+w_{1}-p\right)\right)+\beta_{z} \tilde{\beta}_{1 y n} \tilde{A}_{n} \cos \left(\tilde{\beta}_{1 x n}\left(g_{1}+w_{1}-p\right)\right)\right] \\
& \quad=j \omega \epsilon_{o} \sum_{v=1} \beta_{2 x v}\left[B_{v} \sin \left(\beta_{2 x v} w_{1}\right)-B_{v}^{\prime} \cos \left(\beta_{2 x v} w_{1}\right)\right]\left(I_{1}^{\beta_{2}}+T_{C B} I_{2}^{\beta_{3}}\right) \\
& \quad-j \beta_{z} \sum_{v=1}\left[\tilde{B}_{v} \sin \left(\tilde{\beta}_{2 x v} w_{1}\right)+\tilde{B}_{v}{ }^{\prime} \cos \left(\tilde{\beta}_{2 x v} w_{1}\right)\right]\left(\tilde{\beta}_{2 y v} \tilde{I}_{1}^{\beta_{2}}+\tilde{\beta}_{3 y v} \tilde{T}_{C B} \tilde{I}_{2}^{\beta_{3}}\right)
\end{aligned}
$$

The boundary conditions at $x=-g_{1}+w_{1}$ were enforced:

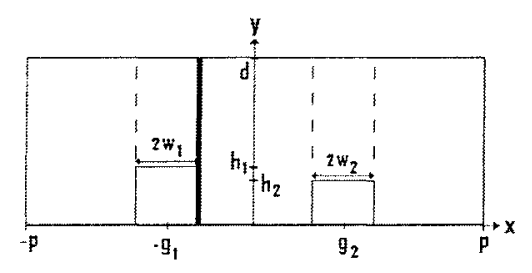


a) Match $E_{y}$ at $x=-g_{1}+w_{1}$ :

$$
\begin{gathered}
\int_{0}^{d} E_{4}^{y} \cos \frac{n \pi y}{d} d y=\int_{0}^{h_{1}} E_{2}^{y} \cos \frac{n \pi y}{d} d y+\int_{h_{1}}^{d} E_{3}^{y} \cos \frac{n \pi y}{d} d y \text { for } \mathrm{n}=0,1 \ldots \mathrm{N}-1 \\
\delta_{c} \frac{d}{2}\left(k_{o}^{2}-\beta_{4 y n}^{2}\right)\left[D_{n} \cos \left(\beta_{4 x n}\left(-g_{1}+w_{1}\right)\right)+D_{n}^{\prime} \sin \left(\beta_{4 x n}\left(-g_{1}+w_{1}\right)\right)\right] \\
\quad=\sum_{v=1}\left(\beta_{z}^{2}+\beta_{2 x v}^{2}\right)\left[B_{v} \cos \left(\beta_{2 x v}\left(-w_{1}\right)\right)+B_{v}^{\prime} \sin \left(\beta_{2 x v}\left(-w_{1}\right)\right)\right]\left(\frac{I_{1}^{\beta_{2}}}{\epsilon_{r 1}}+T_{C B} I_{2}^{\beta_{3}}\right)
\end{gathered}
$$

b) Match $H_{y}$ at $x=-g_{1}+w_{1}$ :

$$
\begin{aligned}
\int_{0}^{d} & H_{4}^{y} \cos \frac{n \pi y}{d} d y=\int_{0}^{h_{1}} H_{2}^{y} \cos \frac{n \pi y}{d} d y+\int_{h_{1}}^{d} H_{3}^{y} \cos \frac{n \pi y}{d} d y \text { for } \mathrm{n}=1,2 \ldots \mathrm{N}-1 \\
& \delta_{s} \frac{d}{2}\left(k_{o}^{2}-\tilde{\beta}_{4 y n}^{2}\right)\left[\tilde{D}_{n} \sin \left(\tilde{\beta}_{4 x n}\left(-g_{1}+w_{1}\right)\right)+{\tilde{D_{n}}}^{\prime} \cos \left(\tilde{\beta}_{4 x n}\left(-g_{1}+w_{1}\right)\right)\right] \\
& =\sum_{v=1}\left(\beta_{z}^{2}+\tilde{\beta}_{2 x v}^{2}\right)\left[\tilde{B}_{v} \sin \left(\tilde{\beta}_{2 x v}\left(-w_{1}\right)\right)+\tilde{B}_{v}^{\prime} \cos \left(\tilde{\beta}_{2 x v}\left(-w_{1}\right)\right)\right]\left(\tilde{I}_{3}^{\beta_{2}}+\tilde{T}_{C B} \tilde{I}_{4}^{\beta_{3}}\right)
\end{aligned}
$$

c) Match $E_{z}$ at $x=-g_{1}+w_{1}$ :

$$
\begin{aligned}
& \int_{0}^{d} E_{4}^{z} \sin \frac{n \pi y}{d} d y=\int_{0}^{h_{1}} E_{2}^{z} \sin \frac{n \pi y}{d} d y+\int_{h_{1}}^{d} E_{3}^{z} \sin \frac{n \pi y}{d} d y \text { for } \mathrm{n}=1,2 \ldots \mathrm{N}-1 \\
& \quad j \delta_{s} \frac{d}{2}\left[\beta_{z} \beta_{4 y n}\left[D_{n} \cos \left(\beta_{4 x n}\left(-g_{1}+w_{1}\right)\right)+D_{n}^{\prime} \sin \left(\beta_{4 x n}\left(-g_{1}+w_{1}\right)\right)\right]\right. \\
& \left.\quad-\omega \mu_{o} \tilde{\beta}_{4 x n}\left[\tilde{D}_{n} \cos \left(\tilde{\beta}_{4 x n}\left(-g_{1}+w_{1}\right)\right)-\tilde{D}_{n}{ }^{\prime} \sin \left(\tilde{\beta}_{4 x n}\left(-g_{1}+w_{1}\right)\right)\right]\right] \\
& \quad=j \beta_{z} \sum_{v=1}\left[B_{v} \cos \left(\beta_{2 x v}\left(-w_{1}\right)\right)+B_{v}^{\prime} \sin \left(\beta_{2 x v}\left(-w_{1}\right)\right)\right]\left(\frac{\beta_{2 y v}}{\epsilon_{r 1}} I_{3}^{\beta_{2}}+\beta_{3 y v} T_{C B} I_{4}^{\beta_{3}}\right) \\
& \quad+j \omega \mu_{o} \sum_{v=1} \tilde{\beta}_{2 x v}\left[\tilde{B}_{v} \cos \left(\tilde{\beta}_{2 x v}\left(-w_{1}\right)\right)-\tilde{B}_{v}{ }^{\prime} \sin \left(\tilde{\beta}_{2 x v}\left(-w_{1}\right)\right)\right]\left(\tilde{I}_{3}^{\beta_{2}}+\tilde{T}_{C B} \tilde{I}_{4}^{\beta_{3}}\right)
\end{aligned}
$$


d) Match $H_{z}$ at $x=-g_{1}+w_{1}$ :

$$
\begin{aligned}
& \int_{0}^{d} H_{4}^{z} \sin \frac{n \pi y}{d} d y=\int_{0}^{h_{1}} H_{2}^{z} \sin \frac{n \pi y}{d} d y+\int_{h_{1}}^{d} H_{3}^{z} \sin \frac{n \pi y}{d} d y \text { for } \mathrm{n}=0,1 \ldots \mathrm{N}-1 \\
& \quad-j \delta_{c} \frac{d}{2}\left[\omega \epsilon_{o} \beta_{4 x n}\left[D_{n} \sin \left(\beta_{4 x n}\left(-g_{1}+w_{1}\right)\right)-D_{n}^{\prime} \cos \left(\beta_{4 x n}\left(-g_{1}+w_{1}\right)\right)\right]\right. \\
& \left.\quad+\beta_{z} \tilde{\beta}_{4 y n}\left[\tilde{D}_{n} \sin \left(\tilde{\beta}_{4 x n}\left(-g_{1}+w_{1}\right)\right)+\tilde{D}_{n}{ }^{\prime} \cos \left(\tilde{\beta}_{4 x n}\left(-g_{1}+w_{1}\right)\right)\right]\right] \\
& \quad=j \omega \epsilon_{o} \sum_{v=1} \beta_{2 x v}\left[B_{v} \sin \left(\beta_{2 x v}\left(-w_{1}\right)\right)-B_{v}^{\prime} \cos \left(\beta_{2 x v}\left(-w_{1}\right)\right)\right]\left(I_{1}^{\beta_{2}}+T_{C B} I_{2}^{\beta_{3}}\right) \\
& \quad-j \beta_{z} \sum_{v=1}\left[\tilde{B}_{v} \sin \left(\tilde{\beta}_{2 x v}\left(-w_{1}\right)\right)+\tilde{B}_{v}{ }^{\prime} \cos \left(\tilde{\beta}_{2 x v}\left(-w_{1}\right)\right)\right]\left(\tilde{\beta}_{2 y v} \tilde{I}_{1}^{\beta_{2}}+\tilde{\beta}_{3 y v} \tilde{T}_{C B} \tilde{I}_{2}^{\beta_{3}}\right)
\end{aligned}
$$

The boundary conditions at $x=g_{2}-w_{2}$ were enforced:

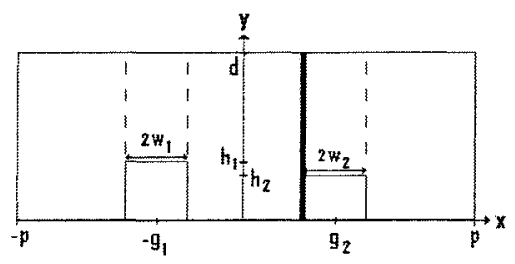

a) Match $E_{y}$ at $x=g_{2}-w_{2}$ :

$$
\begin{aligned}
\int_{0}^{d} & E_{4}^{y} \cos \frac{n \pi y}{d} d y=\int_{0}^{h_{1}} E_{5}^{y} \cos \frac{n \pi y}{d} d y+\int_{h_{1}}^{d} E_{6}^{y} \cos \frac{n \pi y}{d} d y \text { for } \mathrm{n}=0,1 \ldots \mathrm{N}-1 \\
& \delta_{c} \frac{d}{2}\left(k_{o}^{2}-\beta_{4 y n}^{2}\right)\left[D_{n} \cos \left(\beta_{4 x n}\left(g_{2}-w_{2}\right)\right)+D_{n}^{\prime} \sin \left(\beta_{4 x n}\left(g_{2}-w_{2}\right)\right)\right] \\
& =\sum_{v=1}\left(\beta_{z}^{2}+\beta_{5 x v}^{2}\right)\left[E_{v} \cos \left(\beta_{5 x v}\left(-w_{2}\right)\right)+E_{v}^{\prime} \sin \left(\beta_{5 x v}\left(-w_{2}\right)\right)\right]\left(\frac{I_{1}^{\beta_{5}}}{\epsilon_{r 2}}+T_{F E} I_{2}^{\beta_{6}}\right)
\end{aligned}
$$


b) Match $H_{y}$ at $x=g_{2}-w_{2}$ :

$$
\begin{aligned}
\int_{0}^{d} & H_{4}^{y} \sin \frac{n \pi y}{d} d y=\int_{0}^{h_{1}} H_{5}^{y} \sin \frac{n \pi y}{d} d y+\int_{h_{1}}^{d} H_{6}^{y} \sin \frac{n \pi y}{d} d y \text { for } \mathrm{n}=1,2 \ldots \mathrm{N}-1 \\
& \delta_{s} \frac{d}{2}\left(k_{o}^{2}-\tilde{\beta}_{4 y n}^{2}\right)\left[\tilde{D}_{n} \sin \left(\tilde{\beta}_{4 x n}\left(g_{2}-w_{2}\right)\right)+\tilde{D}_{n}^{\prime} \cos \left(\tilde{\beta}_{4 x n}\left(g_{2}-w_{2}\right)\right)\right] \\
& =\sum_{v=1}\left(\beta_{z}^{2}+\tilde{\beta}_{5 x v}^{2}\right)\left[\tilde{E}_{v} \sin \left(\tilde{\beta}_{5 x v}\left(-w_{2}\right)\right)+\tilde{E}_{v}{ }^{\prime} \cos \left(\tilde{\beta}_{5 x v}\left(-w_{2}\right)\right)\right]\left(\tilde{I}_{3}^{\beta_{5}}+\tilde{T}_{F E} \tilde{I}_{4}^{\beta_{6}}\right)
\end{aligned}
$$

c) Match $E_{z}$ at $x=g_{2}-w_{2}$ :

$$
\begin{aligned}
\int_{0}^{d} & E_{4}^{z} \sin \frac{n \pi y}{d} d y=\int_{0}^{h_{1}} E_{5}^{z} \sin \frac{n \pi y}{d} d y+\int_{h_{1}}^{d} E_{6}^{z} \sin \frac{n \pi y}{d} d y \text { for } \mathrm{n}=1,2 \ldots \mathrm{N}-1 \\
& j \delta_{s} \frac{d}{2}\left[\beta_{z} \beta_{4 y n}\left[D_{n} \cos \left(\beta_{4 x n}\left(g_{2}-w_{2}\right)\right)+D_{n}^{\prime} \sin \left(\beta_{4 x n}\left(g_{2}-w_{2}\right)\right)\right]\right. \\
& \left.-\omega \mu_{o} \tilde{\beta}_{4 x n}\left[\tilde{D}_{n} \cos \left(\tilde{\beta}_{4 x n}\left(g_{2}-w_{2}\right)\right)-\tilde{D}_{n}^{\prime} \sin \left(\tilde{\beta}_{4 x n}\left(g_{2}-w_{2}\right)\right)\right]\right] \\
& =j \beta_{z} \sum_{v=1}\left[E_{v} \cos \left(\beta_{5 x v}\left(-w_{2}\right)\right)+E_{v}^{\prime} \sin \left(\beta_{5 x v}\left(-w_{2}\right)\right)\right]\left(\frac{\beta_{5 y v}}{\epsilon_{r 2}} I_{3}^{\beta_{5}}+\beta_{6 y v} T_{F E} I_{4}^{\beta_{6}}\right) \\
& -j \omega \mu_{o} \sum_{v=1} \tilde{\beta}_{5 x v}\left[\tilde{E}_{v} \cos \left(\tilde{\beta}_{5 x v}\left(w_{2}+g_{2}\right)\right)+\tilde{E}_{v}^{\prime} \sin \left(\tilde{\beta}_{5 x v}\left(w_{2}+g_{2}\right)\right)\right]\left(\tilde{I}_{3}^{\beta_{5}}+\tilde{T}_{F E} \tilde{I}_{4}^{\beta_{6}}\right)
\end{aligned}
$$

d) Match $H_{z}$ at $x=g_{2}-w_{2}$ :

$$
\begin{aligned}
& \int_{0}^{d} H_{4}^{z} \cos \frac{n \pi y}{d} d y=\int_{0}^{h_{1}} H_{5}^{z} \cos \frac{n \pi y}{d} d y+\int_{h_{1}}^{d} H_{6}^{z} \cos \frac{n \pi y}{d} d y \text { for } \mathrm{n}=0,1 \ldots \mathrm{N}-1 \\
& \quad-j \delta_{c} \frac{d}{2}\left[\omega \epsilon_{0} \beta_{4 x n}\left[D_{n} \sin \left(\beta_{4 x n}\left(g_{2}-w_{2}\right)\right)-D_{n}^{\prime} \cos \left(\beta_{4 x n}\left(g_{2}-w_{2}\right)\right)\right]\right. \\
& \left.\quad+\beta_{z} \tilde{\beta}_{4 y n}\left[\tilde{D}_{n} \sin \left(\tilde{\beta}_{4 x n}\left(g_{2}-w_{2}\right)\right)+\tilde{D}_{n}{ }^{\prime} \cos \left(\tilde{\beta}_{4 x n}\left(g_{2}-w_{2}\right)\right)\right]\right] \\
& \quad=-j \omega \epsilon_{o} \sum_{v=1} \beta_{5 x v}\left[E_{v} \sin \left(\beta_{5 x v}\left(-w_{2}\right)\right)-E_{v}^{\prime} \cos \left(\beta_{5 x v}\left(-w_{2}\right)\right)\right]\left(I_{1}^{\beta_{5}}+T_{F E} I_{2}^{\beta_{6}}\right) \\
& \quad-j \beta_{z} \sum_{v=1}\left[\tilde{E}_{v} \sin \left(\tilde{\beta}_{5 x v}\left(-w_{2}\right)\right)+\tilde{E}_{v}{ }^{\prime} \cos \left(\tilde{\beta}_{5 x v}\left(-w_{2}\right)\right)\right]\left(\tilde{\beta}_{5 y v} \tilde{I}_{1}^{\beta_{5}}+\tilde{\beta}_{6 y v} \tilde{T}_{F E} \tilde{I}_{2}^{\beta_{6}}\right)
\end{aligned}
$$


The boundary conditions at $x=g_{2}+w_{2}$ were enforced:

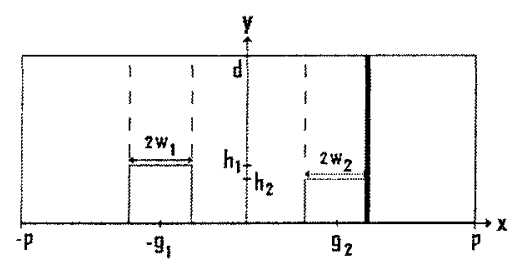

a) Match $E_{y}$ at $x=g_{2}+w_{2}$ :

$$
\begin{aligned}
\int_{0}^{d} & E_{7}^{y} \cos \frac{n \pi y}{d} d y=\int_{0}^{h_{1}} E_{5}^{y} \cos \frac{n \pi y}{d} d y+\int_{h_{1}}^{d} E_{6}^{y} \cos \frac{n \pi y}{d} d y \text { for } \mathrm{n}=0,1 \ldots \mathrm{N}-1 \\
& \delta_{c} \frac{d}{2}\left(\beta_{z}^{2}+\beta_{7 x n}^{2}\right) G_{n} \sin \left(\beta_{7 x n}\left(g_{2}+w_{2}-p\right)\right) \\
& =\sum_{v=1}\left(\beta_{z}^{2}+\beta_{5 x v}^{2}\right)\left[E_{v} \cos \left(\beta_{5 x v}\left(w_{2}\right)\right)+E_{v}^{\prime} \sin \left(\beta_{5 x v}\left(w_{2}\right)\right)\right]\left(\frac{I_{1}^{\beta_{5}}}{\epsilon_{r 2}}+T_{F E} I_{2}^{\beta_{6}}\right)
\end{aligned}
$$

b) Match $H_{y}$ at $x=g_{2}+w_{2}$ :

$$
\begin{gathered}
\int_{0}^{d} H_{7}^{y} \sin \frac{n \pi y}{d} d y=\int_{0}^{h_{1}} H_{5}^{y} \sin \frac{n \pi y}{d} d y+\int_{h_{1}}^{d} H_{6}^{y} \sin \frac{n \pi y}{d} d y \text { for } \mathrm{n}=1,2 \ldots \mathrm{N}-1 \\
\delta_{s} \frac{d}{2}\left(\beta_{z}^{2}+\tilde{\beta}_{7 x n}^{2}\right) \tilde{G}_{n} \cos \left(\tilde{\beta}_{7 x n}\left(g_{2}+w_{2}-p\right)\right) \\
\quad=\sum_{v=1}\left(\beta_{z}^{2}+\tilde{\beta}_{5 x v}^{2}\right)\left[\tilde{E}_{v} \sin \left(\tilde{\beta}_{5 x v}\left(w_{2}\right)\right)+\tilde{E}_{v}^{\prime} \cos \left(\tilde{\beta}_{5 x v}\left(w_{2}\right)\right)\right]\left(\tilde{I}_{3}^{\beta_{5}}+\tilde{T}_{F E} \tilde{I}_{4}^{\beta_{6}}\right)
\end{gathered}
$$


c) Match $E_{z}$ at $x=g_{2}+w_{2}$ :

$$
\begin{aligned}
& \int_{0}^{d} \quad E_{7}^{z} \sin \frac{n \pi y}{d} d y=\int_{0}^{h_{1}} E_{5}^{z} \sin \frac{n \pi y}{d} d y+\int_{h_{1}}^{d} E_{6}^{z} \sin \frac{n \pi y}{d} d y \text { for } \mathrm{n}=1,2 \ldots \mathrm{N}-1 \\
& \quad j \delta_{s} \frac{d}{2}\left[\beta_{z} \beta_{7 y n} G_{n} \sin \left(\beta_{7 x n}\left(g_{2}+w_{2}-p\right)\right)\right. \\
& \left.\quad+\omega \mu_{o} \tilde{\beta}_{7 x n} \tilde{G}_{n} \sin \left(\tilde{\beta}_{7 x n}\left(g_{2}+w_{2}-p\right)\right)\right] \\
& \quad=j \beta_{z} \sum_{v=1}\left[E_{v} \cos \left(\beta_{5 x v}\left(w_{2}\right)\right)+E_{v}^{\prime} \sin \left(\beta_{5 x v}\left(w_{2}\right)\right)\right]\left(\frac{\beta_{5 y v}}{\epsilon_{r 2}} I_{3}^{\beta_{5}}+\beta_{6 y v} T_{F E} I_{4}^{\beta_{6}}\right) \\
& \quad-j \omega \mu_{o} \sum_{v=1} \tilde{\beta}_{5 x v}\left[\tilde{E}_{v} \cos \left(\tilde{\beta}_{5 x v}\left(w_{2}\right)\right)-\tilde{E}_{v}{ }^{\prime} \sin \left(\tilde{\beta}_{5 x v}\left(w_{2}\right)\right)\right]\left(\tilde{I}_{3}^{\beta_{5}}+\tilde{T}_{F E} \tilde{I}_{4}^{\beta_{6}}\right)
\end{aligned}
$$

d) Match $H_{z}$ at $x=g_{2}+w_{2}$ :

$$
\begin{aligned}
\int_{0}^{d} & H_{7}^{z} \cos \frac{n \pi y}{d} d y=\int_{0}^{h_{1}} H_{5}^{z} \cos \frac{n \pi y}{d} d y+\int_{h_{1}}^{d} H_{6}^{z} \cos \frac{n \pi y}{d} d y \text { for } \mathrm{n}=0,1 \ldots \mathrm{N}-1 \\
& j \delta_{c} \frac{d}{2}\left[\omega \epsilon_{o} \beta_{7 x n} G_{n} \cos \left(\beta_{7 x n}\left(g_{2}+w_{2}-p\right)\right)\right. \\
& \left.-\beta_{z} \tilde{\beta}_{7 y n} \tilde{G}_{n} \cos \left(\tilde{\beta}_{7 x n}\left(g_{2}+w_{2}-p\right)\right)\right] \\
& =-j \omega \epsilon_{o} \sum_{v=1} \beta_{5 x v}\left[E_{v} \sin \left(\beta_{5 x v}\left(w_{2}\right)\right)-E_{v}^{\prime} \cos \left(\beta_{5 x v}\left(w_{2}\right)\right)\right]\left(I_{1}^{\beta_{5}}+T_{F E} I_{2}^{\beta_{6}}\right) \\
& -j \beta_{z} \sum_{v=1}\left[\tilde{E}_{v} \sin \left(\tilde{\beta}_{5 x v}\left(w_{2}\right)\right)+\tilde{E}_{v}^{\prime} \cos \left(\tilde{\beta}_{5 x v}\left(w_{2}\right)\right)\right]\left(\tilde{\beta}_{5 y v} \tilde{I}_{1}^{\beta_{5}}+\tilde{T}_{F E} \tilde{I}_{2}^{\beta_{6}}\right)
\end{aligned}
$$

Manipulation of the boundary conditions at $x=-g_{1}-w_{1}$.

Isolate for $A_{n}$ in a) and substitute it into c). Same procedure for $\tilde{A}_{n}$ from b). note: $\beta_{1 x n}$ is always imag, and $\mathrm{p} \rightarrow \infty$. 


$$
\begin{aligned}
& j \frac{\delta_{s}}{\delta_{c}} \frac{\beta_{z} \beta_{1 y n}}{\left(k_{o}^{2}-\beta_{1 y n}^{2}\right)} \sum_{v=1}\left(\beta_{z}^{2}+\beta_{2 x v}^{2}\right)\left[B_{v} \cos \left(\beta_{2 x v} w_{1}\right)+B_{v}^{\prime} \sin \left(\beta_{2 x v} w_{1}\right)\right]\left(I_{1}^{\beta_{2}}+T_{C B} I_{2}^{\beta_{3}}\right) \\
& -\omega \mu_{o} \frac{\tilde{\beta}_{1 x n}}{\left(k_{o}^{2}-\tilde{\beta}_{1 y n}^{2}\right)} \sum_{v=1}\left(\beta_{z}^{2}+\tilde{\beta}_{2 x v}^{2}\right)\left(\tilde{B}_{v} \sin \left(\tilde{\beta}_{2 x v} w_{1}\right)+\tilde{B}_{v}^{\prime} \cos \left(\tilde{\beta}_{2 x v} w_{1}\right)\right)\left(\tilde{I}_{3}^{\beta_{2}}+\tilde{T}_{C B} \tilde{I}_{4}^{\beta_{3}}\right) \\
& =j \beta_{z} \sum_{v=1}\left[B_{v} \cos \left(\beta_{2 x v} w_{1}\right)+B_{v}^{\prime} \sin \left(\beta_{2 x v} w_{1}\right)\right]\left(\frac{\beta_{2 y v}}{\epsilon_{r 1}} I_{3}^{\beta_{2}}+\beta_{3 y v} T_{C B} I_{4}^{\beta_{3}}\right) \\
& +j \omega \mu_{o} \sum_{v=1} \tilde{\beta}_{2 x v}\left[\tilde{B}_{v} \cos \left(\tilde{\beta}_{2 x v} w_{1}\right)-\tilde{B}_{v}^{\prime} \sin \left(\tilde{\beta}_{2 x v} w_{1}\right)\right]\left(\tilde{I}_{3}^{\beta_{2}}+\tilde{T}_{C B} \tilde{I}_{4}^{\beta_{3}}\right) \\
& \text { for } n=1,2,3 \ldots
\end{aligned}
$$

Isolate for $A_{n}$ in a) and substitute it into d). Same procedure for $\tilde{A}_{n}$ from b). note: $\beta_{1 x n}$ is always imag, and $\mathrm{p} \rightarrow \infty$.

$$
\begin{aligned}
& \omega \epsilon_{o} \frac{\beta_{1 x n}}{\left(k_{o}^{2}-\beta_{1 y n}^{2}\right)} \sum_{v=1}\left(\beta_{z}^{2}+\beta_{2 x v}^{2}\right)\left[B_{v} \cos \left(\beta_{2 x v} w_{1}\right)+B_{v}^{\prime} \sin \left(\beta_{2 x v} w_{1}\right)\right]\left(I_{1}^{\beta_{2}}+T_{C B} I_{2}^{\beta_{3}}\right) \\
& -j \frac{\delta_{c}}{\delta_{s}} \frac{\beta_{z} \tilde{\beta}_{1 y n}}{\left(k_{o}^{2}-\tilde{\beta}_{1 y n}^{2}\right)} \sum_{v=1}\left(\beta_{z}^{2}+\tilde{\beta}_{2 x v}^{2}\right)\left(\tilde{B}_{v} \sin \left(\tilde{\beta}_{2 x v} w_{1}\right)+\tilde{B}_{v}^{\prime} \cos \left(\tilde{\beta}_{2 x v} w_{1}\right)\right)\left(\tilde{I}_{3}^{\beta_{2}}+\tilde{T}_{C B} \tilde{I}_{4}^{\beta_{3}}\right) \\
& =j \omega \epsilon_{o} \sum_{v=1} \beta_{2 x v}\left[B_{v} \sin \left(\beta_{2 x v} w_{1}\right)-B_{v}^{\prime} \cos \left(\beta_{2 x v} w_{1}\right)\right]\left(I_{1}^{\beta_{2}}+T_{C B} I_{2}^{\beta_{3}}\right) \\
& -j \beta_{z} \sum_{v=1}\left[\tilde{B}_{v} \sin \left(\tilde{\beta}_{2 x v} w_{1}\right)+\tilde{B}_{v}{ }^{\prime} \cos \left(\tilde{\beta}_{2 x v} w_{1}\right)\right]\left(\tilde{\beta}_{2 y v} \tilde{I}_{1}^{\beta_{2}}+\tilde{\beta}_{2 y v} \tilde{T}_{C B} \tilde{I}_{2}^{\beta_{3}}\right)
\end{aligned}
$$

for $n=0,1,2 \ldots$

The same procedure was used to manipulate the boundary condition equations at $x=g_{2}+w_{2}$. 
First manipulation of the boundary conditions at $x=-g_{1}+w_{1}$ and at $x=g_{2}-w_{2}$. Note: $\left(g_{2}-w_{2}\right)=-\left(-g_{1}+w_{1}\right)$.

i) Sum the two c) conditions:

$$
\begin{aligned}
& j \delta_{s} d\left(\beta_{z} \beta_{4 y n} D_{n} \cos \left(\beta_{4 x n}\left(g_{2}-w_{2}\right)\right)-\omega \mu_{o} \tilde{\beta}_{4 x n} \tilde{D}_{n} \cos \left(\tilde{\beta}_{4 x n}\left(g_{2}-w_{2}\right)\right)\right) \\
& =j \beta_{z} \sum_{v=1}\left[E_{v} \cos \left(\beta_{5 x v}\left(-w_{2}\right)\right)+E_{v}^{\prime} \sin \left(\beta_{5 x v}\left(-w_{2}\right)\right)\right]\left(\frac{\beta_{5 y v}}{\epsilon_{r 2}} I_{3}^{\beta_{5}}+\beta_{6 y v} T_{F E} I_{4}^{\beta_{6}}\right) \\
& -j \omega \mu_{o} \sum_{v=1} \tilde{\beta}_{5 x v}\left[\tilde{E}_{v} \cos \left(\tilde{\beta}_{5 x v}\left(-w_{2}-g_{2}\right)\right)-\tilde{E}_{v}^{\prime} \sin \left(\tilde{\beta}_{5 x v}\left(-w_{2}-g_{2}\right)\right)\right]\left(\tilde{I}_{3}^{\beta_{5}}+\tilde{T}_{F E} \tilde{I}_{4}^{\beta_{6}}\right) \\
& +j \beta_{z} \sum_{v=1}\left[B_{v} \cos \left(\beta_{2 x v}\left(-w_{1}\right)\right)+B_{v}^{\prime} \sin \left(\beta_{2 x v}\left(-w_{1}\right)\right)\right]\left(\frac{\beta_{2 y v}}{\epsilon_{r 1}} I_{3}^{\beta_{2}}+\beta_{3 y v} T_{C B} I_{4}^{\beta_{3}}\right) \\
& +j \omega \mu_{o} \sum_{v=1} \tilde{\beta}_{2 x v}\left[\tilde{B}_{v} \cos \left(\tilde{\beta}_{2 x v}\left(-w_{1}\right)\right)-\tilde{B}_{v}^{\prime} \sin \left(\tilde{\beta}_{2 x v}\left(-w_{1}\right)\right)\right]\left(\tilde{I}_{3}^{\beta_{2}}+\tilde{T}_{C B} \tilde{I}_{4}^{\beta_{3}}\right) \\
& \text { for } n=1,2,3 \ldots
\end{aligned}
$$

ii) Subtract the d) condition at $x=-g_{1}+w_{1}$ from the d) condition at

$$
x=g_{2}-w_{2}:
$$

$$
\begin{aligned}
& -j \delta_{c} d\left(\omega \epsilon_{o} \beta_{4 x n} D_{n} \sin \left(\beta_{4 x n}\left(g_{2}-w_{2}\right)\right)+\beta_{z} \tilde{\beta}_{4 y n} \tilde{D}_{n} \sin \left(\tilde{\beta}_{4 x n}\left(g_{2}-w_{2}\right)\right)\right) \\
& =-j \omega \epsilon_{o} \sum_{v=1} \beta_{5 x v}\left[E_{v} \sin \left(\beta_{5 x v}\left(-w_{2}\right)\right)-E_{v}^{\prime} \cos \left(\beta_{5 x v}\left(-w_{2}\right)\right)\right]\left(I_{1}^{\beta_{5}}+T_{F E} I_{2}^{\beta_{6}}\right) \\
& -j \beta_{z} \sum_{v=1}\left[\tilde{E}_{v} \sin \left(\tilde{\beta}_{5 x v}\left(-w_{2}\right)\right)+\tilde{E}_{v}^{\prime} \cos \left(\tilde{\beta}_{5 x v}\left(-w_{2}\right)\right)\right]\left(\tilde{\beta}_{5 y v} \tilde{I}_{1}^{\beta_{5}}+\tilde{\beta}_{6 y v} \tilde{T}_{F E} \tilde{I}_{2}^{\beta_{6}}\right) \\
& -j \omega \epsilon_{o} \sum_{v=1} \beta_{2 x v}\left[B_{v} \sin \left(\beta_{2 x v}\left(-w_{1}\right)\right)-B_{v}^{\prime} \cos \left(\beta_{2 x v}\left(-w_{1}\right)\right)\right]\left(I_{1}^{\beta_{2}}+T_{C B} I_{2}^{\beta_{3}}\right) \\
& +j \beta_{z} \sum_{v=1}\left[\tilde{B}_{v} \sin \left(\tilde{\beta}_{2 x v}\left(-w_{1}\right)\right)+\tilde{B}_{v}{ }^{\prime} \cos \left(\tilde{\beta}_{2 x v}\left(-w_{1}\right)\right)\right]\left(\tilde{\beta}_{2 y v} \tilde{I}_{1}^{\beta_{2}}+\tilde{\beta}_{3 y v} \tilde{T}_{C B} \tilde{I}_{2}^{\beta_{3}}\right)
\end{aligned}
$$

for $n=0,1,2 \ldots$ 
iii) Sum the two a) conditions:

$$
\begin{aligned}
& \delta_{c} d\left(k_{o}^{2}-\beta_{4 y n}^{2}\right) D_{n} \cos \left(\beta_{4 x n}\left(g_{2}-w_{2}\right)\right) \\
& =\sum_{v=1}\left(\beta_{z}^{2}+\beta_{5 x v}^{2}\right)\left[E_{v} \cos \left(\beta_{5 x v}\left(-w_{2}\right)\right)+E_{v}^{\prime} \sin \left(\beta_{5 x v}\left(-w_{2}\right)\right)\right]\left(\frac{I_{1}^{\beta_{5}}}{\epsilon_{r 2}}+T_{F E} I_{2}^{\beta_{6}}\right) \\
& +\sum_{v=1}\left(\beta_{z}^{2}+\beta_{2 x v}^{2}\right)\left[B_{v} \cos \left(\beta_{2 x v}\left(-w_{1}\right)\right)+B_{v}^{\prime} \sin \left(\beta_{2 x v}\left(-w_{1}\right)\right)\right]\left(\frac{I_{1}^{\beta_{2}}}{\epsilon_{r 1}}+T_{C B} I_{2}^{\beta_{3}}\right) \\
& \text { for } n=0,1,2 \ldots
\end{aligned}
$$

iv) Subtract the b) condition at $x=-g_{1}+w_{1}$ from the b) condition at $x=g_{2}-w_{2}:$

$$
\begin{aligned}
& \delta_{s} d\left(k_{o}^{2}-\tilde{\beta}_{4 y n}^{2}\right) \tilde{D}_{n} \sin \left(\tilde{\beta}_{4 x n}\left(g_{2}-w_{2}\right)\right) \\
& =\sum_{v=1}\left(\beta_{z}^{2}+\tilde{\beta}_{5 x v}^{2}\right)\left[\tilde{E}_{v} \sin \left(\tilde{\beta}_{5 x v}\left(-w_{2}\right)\right)+\tilde{E}_{v}^{\prime} \cos \left(\tilde{\beta}_{5 x v}\left(-w_{2}\right)\right)\right]\left(\tilde{I}_{3}^{\beta_{5}}+\tilde{T}_{F E} \tilde{I}_{4}^{\beta_{6}}\right) \\
& -\sum_{v=1}\left(\beta_{z}^{2}+\tilde{\beta}_{2 x v}^{2}\right)\left[\tilde{B}_{v} \sin \left(\tilde{\beta}_{2 x v}\left(-w_{1}\right)\right)+\tilde{B}_{v}^{\prime} \cos \left(\tilde{\beta}_{2 x v}\left(-w_{1}\right)\right)\right]\left(\tilde{I}_{3}^{\beta_{2}}+\tilde{T}_{C B} \tilde{I}_{4}^{\beta_{3}}\right) \\
& \text { for } n=1,2,3 \ldots
\end{aligned}
$$


v) Isolate $D_{n}$ in iii) and $\tilde{D}_{n}$ in iv) and sub them both into i):

$$
\begin{aligned}
& j \frac{\delta_{s}}{\delta_{c}} \frac{\beta_{z} \beta_{4 y n}}{\left(k_{o}^{2}-\beta_{4 y n}^{2}\right)}\left[\sum_{v=1}\left(\beta_{z}^{2}+\beta_{5 x v}^{2}\right)\left[E_{v} \cos \left(\beta_{5 x v}\left(-w_{2}\right)\right)+E_{v}^{\prime} \sin \left(\beta_{5 x v}\left(-w_{2}\right)\right)\right]\right. \\
& \times\left(\frac{I_{1}^{\beta_{5}}}{\epsilon_{r 2}}+T_{F E} I_{2}^{\beta_{6}}\right)+\sum_{v=1}\left(\beta_{z}^{2}+\beta_{2 x v}^{2}\right)\left[B_{v} \cos \left(\beta_{2 x v}\left(-w_{1}\right)\right)+B_{v}^{\prime} \sin \left(\beta_{2 x v}\left(-w_{1}\right)\right)\right] \\
& \left.\left.\left(\frac{I_{1}^{\beta_{2}}}{\epsilon_{r 1}}+T_{C B} I_{2}^{\beta_{3}}\right)\right]-j \omega \mu_{o} \frac{\tilde{\beta}_{4 x n}}{\left(k_{o}^{2}-\tilde{\beta}_{4 y n}^{2}\right)} \cot \left(\tilde{\beta}_{4 x n}\left(g_{2}-w_{2}\right)\right)\right) \\
& \left(\sum_{v=1}\left(\beta_{z}^{2}+\tilde{\beta}_{5 x v}^{2}\right)\left[\tilde{E}_{v} \sin \left(\tilde{\beta}_{5 x v}\left(-w_{2}\right)\right)+\tilde{E}_{v}{ }^{\prime} \cos \left(\tilde{\beta}_{5 x v}\left(-w_{2}\right)\right)\right]\left(\tilde{I}_{3}^{\beta_{5}}+\tilde{T}_{F E} \tilde{I}_{4}^{\beta_{6}}\right)\right. \\
& \left.-\sum_{v=1}\left(\beta_{z}^{2}+\tilde{\beta}_{2 x v}^{2}\right)\left[\tilde{B}_{v} \sin \left(\tilde{\beta}_{2 x v}\left(-w_{1}\right)\right)+\tilde{B}_{v}^{\prime} \cos \left(\tilde{\beta}_{2 x v}\left(-w_{1}\right)\right)\right]\left(\tilde{I}_{3}^{\beta_{2}}+\tilde{T}_{C B} \tilde{I}_{4}^{\beta_{3}}\right)\right) \\
& =j \beta_{z} \sum_{v=1}\left[E_{v} \cos \left(\beta_{5 x v}\left(-w_{2}\right)\right)+E_{v}^{\prime} \sin \left(\beta_{5 x v}\left(-w_{2}\right)\right)\right]\left(\frac{\beta_{5 y v}}{\epsilon_{r 2}} I_{3}^{\beta_{5}}+\beta_{6 y v} T_{F E} I_{4}^{\beta_{6}}\right) \\
& -j \omega \mu_{o} \sum_{v=1} \tilde{\beta}_{5 x v}\left[\tilde{E}_{v} \cos \left(\tilde{\beta}_{5 x v}\left(x-g_{2}\right)\right)-\tilde{E}_{v}{ }^{\prime} \sin \left(\tilde{\beta}_{5 x v}\left(x-g_{2}\right)\right)\right]\left(\tilde{I}_{3}^{\beta_{5}}+\tilde{T}_{F E} \tilde{I}_{4}^{\beta_{6}}\right) \\
& +j \beta_{z} \sum_{v=1}\left[B_{v} \cos \left(\beta_{2 x v}\left(-w_{1}\right)\right)+B_{v}^{\prime} \sin \left(\beta_{2 x v}\left(-w_{1}\right)\right)\right]\left(\frac{\beta_{2 y v}}{\epsilon_{r 1}} I_{3}^{\beta_{2}}+\beta_{3 y v} T_{C B} I_{4}^{\beta_{3}}\right) \\
& +j \omega \mu_{o} \sum_{v=1} \tilde{\beta}_{2 x v}\left[\tilde{B}_{v} \cos \left(\tilde{\beta}_{2 x v}\left(-w_{1}\right)\right)-\tilde{B}_{v}{ }^{\prime} \sin \left(\tilde{\beta}_{2 x v}\left(-w_{1}\right)\right)\right]\left(\tilde{I}_{3}^{\beta_{2}}+\tilde{T}_{C B} \tilde{I}_{4}^{\beta_{3}}\right) \\
& \text { for } n=0,1,2 \ldots
\end{aligned}
$$


vi) Isolate $D_{n}$ in iii) and $\tilde{D}_{n}$ in iv) and sub them both into ii):

$$
\begin{aligned}
& -j \omega \epsilon_{o} \frac{\beta_{4 x n}}{\left(k_{o}^{2}-\beta_{4 y n}^{2}\right)} \tan \left(\beta_{4 x n}\left(g_{2}-w_{2}\right)\right)\left[\sum _ { v = 1 } ( \beta _ { z } ^ { 2 } + \beta _ { 5 x v } ^ { 2 } ) \left[E_{v} \cos \left(\beta_{5 x v}\left(-w_{2}\right)\right)\right.\right. \\
& \left.+E_{v}^{\prime} \sin \left(\beta_{5 x v}\left(-w_{2}\right)\right)\right]\left(\frac{I_{1}^{\beta_{5}}}{\epsilon_{r 2}}+T_{F E} I_{2}^{\beta_{6}}\right)+\sum_{v=1}\left(\beta_{z}^{2}+\beta_{2 x v}^{2}\right)\left[B_{v} \cos \left(\beta_{2 x v}\left(-w_{1}\right)\right)\right. \\
& \left.\left.+B_{v}^{\prime} \sin \left(\beta_{2 x v}\left(-w_{1}\right)\right)\right]\left(\frac{I_{1}^{\beta_{2}}}{\epsilon_{r 1}}+T_{C B} I_{2}^{\beta_{3}}\right)\right]-j \frac{\delta_{c}}{\delta_{s}} \frac{\beta_{z} \tilde{\beta}_{4 y n}}{\left(k_{o}^{2}-\tilde{\beta}_{4 y n}^{2}\right)}\left[\sum_{v=1}\left(\beta_{z}^{2}+\tilde{\beta}_{5 x v}^{2}\right)\right. \\
& \times\left[\tilde{E}_{v} \sin \left(\tilde{\beta}_{5 x v}\left(-w_{2}\right)\right)+\tilde{E}_{v}{ }^{\prime} \cos \left(\tilde{\beta}_{5 x v}\left(-w_{2}\right)\right)\right]\left(\tilde{I}_{3}^{\beta_{5}}+\tilde{T}_{F E} \tilde{I}_{4}^{\beta_{6}}\right) \\
& \left.-\sum_{v=1}\left(\beta_{z}^{2}+\tilde{\beta}_{2 x v}^{2}\right)\left[\tilde{B}_{v} \sin \left(\tilde{\beta}_{2 x v}\left(-w_{1}\right)\right)+\tilde{B}_{v}^{\prime} \cos \left(\tilde{\beta}_{2 x v}\left(-w_{1}\right)\right)\right]\left(\tilde{I}_{3}^{\beta_{2}}+\tilde{T}_{C B} \tilde{I}_{4}^{\beta_{3}}\right)\right] \\
& =-j \omega \epsilon_{o} \sum_{v=1} \beta_{5 x v}\left[E_{v} \sin \left(\beta_{5 x v}\left(-w_{2}\right)\right)-E_{v}^{\prime} \cos \left(\beta_{5 x v}\left(-w_{2}\right)\right)\right]\left(I_{1}^{\beta_{5}}+T_{F E} I_{2}^{\beta_{6}}\right) \\
& -j \beta_{z} \sum_{v=1}\left[\tilde{E}_{v} \sin \left(\tilde{\beta}_{5 x v}\left(-w_{2}\right)\right)+\tilde{E}_{v}{ }^{\prime} \cos \left(\tilde{\beta}_{5 x v}\left(-w_{2}\right)\right)\right]\left(\tilde{\beta}_{5 y v} \tilde{I}_{1}^{\beta_{5}}+\tilde{\beta}_{6 y v} \tilde{T}_{F E} \tilde{I}_{2}^{\beta_{6}}\right) \\
& -j \omega \epsilon_{o} \sum_{v=1} \beta_{2 x v}\left[B_{v} \sin \left(\beta_{2 x v}\left(-w_{1}\right)\right)-B_{v}^{\prime} \cos \left(\beta_{2 x v}\left(-w_{1}\right)\right)\right]\left(I_{1}^{\beta_{2}}+T_{C B} I_{2}^{\beta_{3}}\right) \\
& +j \beta_{z} \sum_{v=1}\left[\tilde{B}_{v} \sin \left(\tilde{\beta}_{2 x v}\left(-w_{1}\right)\right)+\tilde{B}_{v}^{\prime} \cos \left(\tilde{\beta}_{2 x v}\left(-w_{1}\right)\right)\right]\left(\tilde{\beta}_{2 y v} \tilde{I}_{1}^{\beta_{2}}+\tilde{\beta}_{3 y v} \tilde{T}_{C B} \tilde{I}_{2}^{\beta_{3}}\right) \\
& \text { for } n=0,1,2 \ldots
\end{aligned}
$$


Second manipulation of the boundary conditions at $x=-g_{1}+w_{1}$ and at $x=g_{2}-w_{2}$. Note: $\left(g_{2}-w_{2}\right)=-\left(-g_{1}+w_{1}\right)$.

i) Subtract the c) condition at $x=-g_{1}+w_{1}$ from the c) condition at $x=g_{2}-w_{2}:$

$$
\begin{aligned}
& j \delta_{s} d\left(\beta_{z} \beta_{4 y n} D_{n}^{\prime} \sin \left(\beta_{4 x n}\left(g_{2}-w_{2}\right)\right)+\omega \mu_{o} \tilde{\beta}_{4 x n} \tilde{D}_{n}^{\prime} \sin \left(\tilde{\beta}_{4 x n}\left(g_{2}-w_{2}\right)\right)\right) \\
& =j \beta_{z} \sum_{v=1}\left[E_{v} \cos \left(\beta_{5 x v}\left(-w_{2}\right)\right)+E_{v}^{\prime} \sin \left(\beta_{5 x v}\left(-w_{2}\right)\right)\right]\left(\frac{\beta_{5 y v}}{\epsilon_{r 2}} I_{3}^{\beta_{5}}+\beta_{6 y v} T_{F E} I_{4}^{\beta_{6}}\right) \\
& -j \omega \mu_{o} \sum_{v=1} \tilde{\beta}_{5 x v}\left[\tilde{E}_{v} \cos \left(\tilde{\beta}_{5 x v}\left(-w_{2}\right)\right)-\tilde{E}_{v}^{\prime} \sin \left(\tilde{\beta}_{5 x v}\left(-w_{2}\right)\right)\right]\left(\tilde{I}_{3}^{\beta_{5}}+\tilde{T}_{F E} \tilde{I}_{4}^{\beta_{6}}\right) \\
& -j \beta_{z} \sum_{v=1}\left[B_{v} \cos \left(\beta_{2 x v}\left(-w_{1}\right)\right)+B_{v}^{\prime} \sin \left(\beta_{2 x v}\left(-w_{1}\right)\right)\right]\left(\frac{\beta_{2 y v}}{\epsilon_{r 1}} I_{3}^{\beta_{2}}+\beta_{3 y v} T_{C B} I_{4}^{\beta_{3}}\right) \\
& -j \omega \mu_{o} \sum_{v=1} \tilde{\beta}_{2 x v}\left[\tilde{B}_{v} \cos \left(\tilde{\beta}_{2 x v}\left(-w_{1}\right)\right)-\tilde{B}_{v}^{\prime} \sin \left(\tilde{\beta}_{2 x v}\left(-w_{1}\right)\right)\right]\left(\tilde{I}_{3}^{\beta_{2}}+\tilde{T}_{C B} \tilde{I}_{4}^{\beta_{3}}\right)
\end{aligned}
$$

for $n=1,2,3 \ldots$

ii) Sum the two d) conditions:

$$
\begin{aligned}
& j \delta_{c} d\left(\omega \epsilon_{o} \beta_{4 x n} D_{n}^{\prime} \cos \left(\beta_{4 x n}\left(g_{2}-w_{2}\right)\right)-\beta_{z} \tilde{\beta}_{4 y n} \tilde{D}_{n}^{\prime} \cos \left(\tilde{\beta}_{4 x n}\left(g_{2}-w_{2}\right)\right)\right) \\
& =-j \omega \epsilon_{o} \sum_{v=1} \beta_{5 x v}\left[E_{v} \sin \left(\beta_{5 x v}\left(-w_{2}\right)\right)-E_{v}^{\prime} \cos \left(\beta_{5 x v}\left(-w_{2}\right)\right)\right]\left(I_{1}^{\beta_{5}}+T_{F E} I_{2}^{\beta_{6}}\right) \\
& -j \beta_{z} \sum_{v=1}\left[\tilde{E}_{v} \sin \left(\tilde{\beta}_{5 x v}\left(-w_{2}\right)\right)+\tilde{E}_{v}^{\prime} \cos \left(\tilde{\beta}_{5 x v}\left(-w_{2}\right)\right)\right]\left(\tilde{\beta}_{5 y v} \tilde{I}_{1}^{\beta_{5}}+\tilde{\beta}_{6 y v} \tilde{T}_{F E} \tilde{I}_{2}^{\beta_{6}}\right) \\
& +j \omega \epsilon_{o} \sum_{v=1} \beta_{2 x v}\left[B_{v} \sin \left(\beta_{2 x v}\left(-w_{1}\right)\right)-B_{v}^{\prime} \cos \left(\beta_{2 x v}\left(-w_{1}\right)\right)\right]\left(I_{1}^{\beta_{2}}+T_{C B} I_{2}^{\beta_{3}}\right) \\
& -j \beta_{z} \sum_{v=1}\left[\tilde{B}_{v} \sin \left(\tilde{\beta}_{2 x v}\left(-w_{1}\right)\right)+\tilde{B}_{v}{ }^{\prime} \cos \left(\tilde{\beta}_{2 x v}\left(-w_{1}\right)\right)\right]\left(\tilde{\beta}_{2 y v} \tilde{I}_{1}^{\beta_{2}}+\tilde{\beta}_{3 y v} \tilde{T}_{C B} \tilde{I}_{2}^{\beta_{3}}\right) \\
& \text { for } n=0,1,2 \ldots
\end{aligned}
$$


iii) Subtract the a) condition at $x=-g_{1}+w_{1}$ from the a) condition at $x=g_{2}-w_{2}:$

$$
\begin{aligned}
& \delta_{c} d\left(k_{o}^{2}-\beta_{4 y n}^{2}\right) D_{n}^{\prime} \sin \left(\beta_{4 x n}\left(g_{2}-w_{2}\right)\right) \\
& =\sum_{v=1}\left(\beta_{z}^{2}+\beta_{5 x v}^{2}\right)\left[E_{v} \cos \left(\beta_{5 x v}\left(-w_{2}\right)\right)+E_{v}^{\prime} \sin \left(\beta_{5 x v}\left(-w_{2}\right)\right)\right]\left(\frac{I_{1}^{\beta_{5}}}{\epsilon_{r 2}}+T_{F E} I_{2}^{\beta_{6}}\right) \\
& -\sum_{v=1}\left(\beta_{z}^{2}+\beta_{2 x v}^{2}\right)\left[B_{v} \cos \left(\beta_{2 x v}\left(-w_{1}\right)\right)+B_{v}^{\prime} \sin \left(\beta_{2 x v}\left(-w_{1}\right)\right)\right]\left(\frac{I_{1}^{\beta_{2}}}{\epsilon_{r 1}}+T_{C B} I_{2}^{\beta_{3}}\right) \\
& \text { for } n=0,1,2 \ldots
\end{aligned}
$$

iv) Sum the two b) conditions:

$$
\begin{aligned}
& \delta_{s} d\left(k_{o}^{2}-\tilde{\beta}_{4 y n}^{2}\right) \tilde{D}_{n}^{\prime} \cos \left(\tilde{\beta}_{4 x n}\left(g_{2}-w_{2}\right)\right) \\
& =\sum_{v=1}\left(\beta_{z}^{2}+\tilde{\beta}_{5 x v}^{2}\right)\left[\tilde{E}_{v} \sin \left(\tilde{\beta}_{5 x v}\left(-w_{2}\right)\right)+\tilde{E}_{v}^{\prime} \cos \left(\tilde{\beta}_{5 x v}\left(-w_{2}\right)\right)\right]\left(\tilde{I}_{3}^{\beta_{5}}+\tilde{T}_{F E} \tilde{I}_{4}^{\beta_{6}}\right) \\
& +\sum_{v=1}\left(\beta_{z}^{2}+\tilde{\beta}_{2 x v}^{2}\right)\left[\tilde{B}_{v} \sin \left(\tilde{\beta}_{2 x v}\left(-w_{1}\right)\right)+\tilde{B}_{v}^{\prime} \cos \left(\tilde{\beta}_{2 x v}\left(-w_{1}\right)\right)\right]\left(\tilde{I}_{3}^{\beta_{2}}+\tilde{T}_{C B} \tilde{I}_{4}^{\beta_{3}}\right) \\
& \text { for } n=1,2,3 \ldots
\end{aligned}
$$


v) Isolate $D_{n}^{\prime}$ in iii) and $\tilde{D}_{n}^{\prime}$ in iv) and sub them both into i):

$$
\begin{aligned}
& j \frac{\delta_{s}}{\delta_{c}} \frac{\beta_{z} \beta_{4 y n}}{\left(k_{o}^{2}-\beta_{4 y n}^{2}\right)}\left[\sum_{v=1}\left(\beta_{z}^{2}+\beta_{5 x v}^{2}\right)\left[E_{v} \cos \left(\beta_{5 x v}\left(-w_{2}\right)\right)+E_{v}^{\prime} \sin \left(\beta_{5 x v}\left(-w_{2}\right)\right)\right]\right. \\
& \times\left(\frac{I_{1}^{\beta_{5}}}{\epsilon_{r 2}}+T_{F E} I_{2}^{\beta_{6}}\right)-\sum_{v=1}\left(\beta_{z}^{2}+\beta_{2 x v}^{2}\right)\left[B_{v} \cos \left(\beta_{2 x v}\left(-w_{1}\right)\right)+B_{v}^{\prime} \sin \left(\beta_{2 x v}\left(-w_{1}\right)\right)\right] \\
& \left.\left.\times\left(\frac{I_{1}^{\beta_{2}}}{\epsilon_{r 1}}+T_{C B} I_{2}^{\beta_{3}}\right)\right]+j \omega \mu_{o} \frac{\tilde{\beta}_{4 x n}}{\left(k_{o}^{2}-\tilde{\beta}_{4 y n}^{2}\right)} \tan \left(\tilde{\beta}_{4 x n}\left(g_{2}-w_{2}\right)\right)\right) \\
& \times\left[\sum_{v=1}\left(\beta_{z}^{2}+\tilde{\beta}_{5 x v}^{2}\right)\left[\tilde{E}_{v} \sin \left(\tilde{\beta}_{5 x v}\left(-w_{2}\right)\right)+\tilde{E}_{v}{ }^{\prime} \cos \left(\tilde{\beta}_{5 x v}\left(-w_{2}\right)\right)\right]\left(\tilde{I}_{3}^{\beta_{5}}+\tilde{T}_{F E} \tilde{I}_{4}^{\beta_{6}}\right)\right. \\
& \left.+\sum_{v=1}\left(\beta_{z}^{2}+\tilde{\beta}_{2 x v}^{2}\right)\left[\tilde{B}_{v} \sin \left(\tilde{\beta}_{2 x v}\left(-w_{1}\right)\right)+\tilde{B}_{v}^{\prime} \cos \left(\tilde{\beta}_{2 x v}\left(-w_{1}\right)\right)\right]\left(\tilde{I}_{3}^{\beta_{2}}+\tilde{T}_{C B} \tilde{I}_{4}^{\beta_{3}}\right)\right] \\
& =j \beta_{z} \sum_{v=1}\left[E_{v} \cos \left(\beta_{5 x v}\left(-w_{2}\right)\right)+E_{v}^{\prime} \sin \left(\beta_{5 x v}\left(-w_{2}\right)\right)\right]\left(\frac{\beta_{5 y v}}{\epsilon_{r 2}} I_{3}^{\beta_{5}}+\beta_{6 y v} T_{F E} I_{4}^{\beta_{6}}\right) \\
& -j \omega \mu_{o} \sum_{v=1} \tilde{\beta}_{5 x v}\left[\tilde{E}_{v} \cos \left(\tilde{\beta}_{5 x v}\left(-w_{2}\right)\right)-\tilde{E}_{v}{ }^{\prime} \sin \left(\tilde{\beta}_{5 x v}\left(-w_{2}\right)\right)\right]\left(\tilde{I}_{3}^{\beta_{5}}+\tilde{T}_{F E} \tilde{I}_{4}^{\beta_{6}}\right) \\
& -j \beta_{z} \sum_{v=1}\left[B_{v} \cos \left(\beta_{2 x v}\left(-w_{1}\right)\right)+B_{v}^{\prime} \sin \left(\beta_{2 x v}\left(-w_{1}\right)\right)\right]\left(\frac{\beta_{2 y v}}{\epsilon_{r 1}} I_{3}^{\beta_{2}}+\beta_{3 y v} T_{C B} I_{4}^{\beta_{3}}\right) \\
& -j \omega \mu_{o} \sum_{v=1} \tilde{\beta}_{2 x v}\left[\tilde{B}_{v} \cos \left(\tilde{\beta}_{2 x v}\left(-w_{1}\right)\right)-\tilde{B}_{v}{ }^{\prime} \sin \left(\tilde{\beta}_{2 x v}\left(-w_{1}\right)\right)\right]\left(\tilde{I}_{3}^{\beta_{2}}+\tilde{T}_{C B} \tilde{I}_{4}^{\beta_{3}}\right)
\end{aligned}
$$


vi) Isolate $D_{n}^{\prime}$ in iii) and $\tilde{D}_{n}^{\prime}$ in iv) and sub them both into ii):

$$
\begin{aligned}
& j \omega \epsilon_{o} \frac{\beta_{4 x n}}{\left(k_{o}^{2}-\beta_{4 y n}^{2}\right)} \cot \left(\beta_{4 x n}\left(g_{2}-w_{2}\right)\right)\left[\sum _ { v = 1 } ( \beta _ { z } ^ { 2 } + \beta _ { 5 x v } ^ { 2 } ) \left[E_{v} \cos \left(\beta_{5 x v}\left(-w_{2}\right)\right)\right.\right. \\
& \left.+E_{v}^{\prime} \sin \left(\beta_{5 x v}\left(-w_{2}\right)\right)\right]\left(\frac{I_{1}^{\beta_{5}}}{\epsilon_{r 2}}+T_{F E} I_{2}^{\beta_{6}}\right)-\sum_{v=1}\left(\beta_{z}^{2}+\beta_{2 x v}^{2}\right)\left[B_{v} \cos \left(\beta_{2 x v}\left(-w_{1}\right)\right)\right. \\
& \left.\left.+B_{v}^{\prime} \sin \left(\beta_{2 x v}\left(-w_{1}\right)\right)\right]\left(\frac{I_{1}^{\beta_{2}}}{\epsilon_{r 1}}+T_{C B} I_{2}^{\beta_{3}}\right)\right]-j \frac{\delta_{c}}{\delta_{s}} \frac{\beta_{z} \tilde{\beta}_{4 y n}}{\left(k_{o}^{2}-\tilde{\beta}_{4 y n}^{2}\right)}\left[\sum_{v=1}\left(\beta_{z}^{2}+\tilde{\beta}_{5 x v}^{2}\right)\right. \\
& \times\left[\tilde{E}_{v} \sin \left(\tilde{\beta}_{5 x v}\left(-w_{2}\right)\right)+\tilde{E}_{v}{ }^{\prime} \cos \left(\tilde{\beta}_{5 x v}\left(-w_{2}\right)\right)\right]\left(\tilde{I}_{3}^{\beta_{5}}+\tilde{T}_{F E} \tilde{I}_{4}^{\beta_{6}}\right) \\
& \left.+\sum_{v=1}\left(\beta_{z}^{2}+\tilde{\beta}_{2 x v}^{2}\right)\left[\tilde{B}_{v} \sin \left(\tilde{\beta}_{2 x v}\left(-w_{1}\right)\right)+\tilde{B}_{v}^{\prime} \cos \left(\tilde{\beta}_{2 x v}\left(-w_{1}\right)\right)\right]\left(\tilde{I}_{3}^{\beta_{2}}+\tilde{T}_{C B} \tilde{I}_{4}^{\beta_{3}}\right)\right] \\
& =-j \omega \epsilon_{o} \sum_{v=1} \beta_{5 x v}\left[E_{v} \sin \left(\beta_{5 x v}\left(-w_{2}\right)\right)-E_{v}^{\prime} \cos \left(\beta_{5 x v}\left(-w_{2}\right)\right)\right]\left(I_{1}^{\beta_{5}}+T_{F E} I_{2}^{\beta_{6}}\right) \\
& -j \beta_{z} \sum_{v=1}\left[\tilde{E}_{v} \sin \left(\tilde{\beta}_{5 x v}\left(-w_{2}\right)\right)+\tilde{E}_{v}{ }^{\prime} \cos \left(\tilde{\beta}_{5 x v}\left(-w_{2}\right)\right)\right]\left(\tilde{\beta}_{5 y v} \tilde{I}_{1}^{\beta_{5}}+\tilde{\beta}_{6 y v} \tilde{T}_{F E} \tilde{I}_{2}^{\beta_{6}}\right) \\
& +j \omega \epsilon_{o} \sum_{v=1} \beta_{2 x v}\left[B_{v} \sin \left(\beta_{2 x v}\left(-w_{1}\right)\right)-B_{v}^{\prime} \cos \left(\beta_{2 x v}\left(-w_{1}\right)\right)\right]\left(I_{1}^{\beta_{2}}+T_{C B} I_{2}^{\beta_{3}}\right) \\
& -j \beta_{z} \sum_{v=1}\left[\tilde{B}_{v} \sin \left(\tilde{\beta}_{2 x v}\left(-w_{1}\right)\right)+\tilde{B}_{v}{ }^{\prime} \cos \left(\tilde{\beta}_{2 x v}\left(-w_{1}\right)\right)\right]\left(\tilde{\beta}_{2 y v} \tilde{I}_{1}^{\beta_{2}}+\tilde{\beta}_{3 y v} \tilde{T}_{C B} \tilde{I}_{2}^{\beta_{3}}\right)
\end{aligned}
$$




\section{Integrals solved in closed form}

These make up the $I$ terms in the derivation. The $\tilde{I}$ terms are the similar, with the only difference being a substitution of $\tilde{\beta}_{y v}$ for all of the $\beta_{y v}$ terms.

$$
\begin{aligned}
I_{1} & =\int_{0}^{h} \cos \left(\beta_{y v} y\right) \cos \frac{n \pi y}{d} d y \\
& =\frac{1}{(n \pi)^{2}-\left(\beta_{y v} d\right)^{2}}\left(d n \pi \cos \left(\beta_{y v} h\right) \sin \frac{n \pi h}{d}-\beta_{y v} d^{2} \sin \left(\beta_{y v} h\right) \cos \frac{n \pi h}{d}\right) \\
I_{2}= & \int_{h}^{d} \cos \left(\beta_{y v}(y-d)\right) \cos \frac{n \pi y}{d} d y \\
& =\frac{-1}{(n \pi)^{2}-\left(\beta_{y v} d\right)^{2}}\left(d n \pi \cos \left(\beta_{y v}(d-h)\right) \sin \frac{n \pi h}{d}+\beta_{y v} d^{2} \sin \left(\beta_{y v}(d-h)\right) \cos \frac{n \pi h}{d}\right) \\
I_{3}= & \int_{0}^{h} \sin \left(\beta_{y v} y\right) \sin \frac{n \pi y}{d} d y \\
= & \frac{-1}{(n \pi)^{2}-\left(\beta_{y v} d\right)^{2}}\left(d n \pi \sin \left(\beta_{y v} h\right) \cos \frac{n \pi h}{d}-\beta_{y v} d^{2} \cos \left(\beta_{y v} h\right) \sin \frac{n \pi h}{d}\right) \\
I_{4}= & \int_{h}^{d} \sin \left(\beta_{y v}(y-d)\right) \sin \frac{n \pi y}{d} d y \\
= & \frac{-1}{(n \pi)^{2}-\left(\beta_{y v} d\right)^{2}}\left(d n \pi \sin \left(\beta_{y v}(d-h)\right) \cos \frac{n \pi h}{d}+\beta_{y v} d^{2} \cos \left(\beta_{y v}(d-h)\right) \sin \frac{n \pi h}{d}\right)
\end{aligned}
$$




\section{Bibliography}

[1] T. Teshirogi and T. Yoneyama, Eds., Modern Millimter-Wave Technologies. Tokyo, Japan: Ohmsha, Ltd., 2001.

[2] M. G. Keller, M. B. Oliver, D. J. Roscoe, R. K. Mongia, Y. M. M. Antar, and A. Ittipiboon, "EHF dielectric resonator antenna array," Microwave and Optical Technology Letters, vol. 17, no. 6, pp. 345-349, Apr. 1998.

[3] Radio spectrum allocations in Canada, Industry Canada Std., 2005.

[4] P. Bhartia and I. J. Bahl, Millimeter Wave Engineering and Applications. New York, NY, USA: John Wiley and Sons, 1984.

[5] M. T. Birand and R. V. Gelsthorpe, "Experimental millimetric array using dielectric radiators fed by means of dielectric waveguide," Electronics Letters, vol. 17, no. 18, pp. 633-635, Sept. 1981.

[6] A. Henderson, E. England, and J. R. James, "New low-loss millimetrewave hybrid microstrip antenna array," in Proc. of the 11th European Microwave Conference, Amsterdam, The Netherlands, Sept. 1981, pp. 825-830.

[7] A. Petosa, R. K. Mongia, A. Ittipiboon, and J. S. Wight, "Investigation of various feed structures for linear arrays of dielectric resonator antennas," in IEEE AP-S Int. Symp. Dig., vol. 4, Newport Beach, CA, June 1995, pp. 1982-1985.

[8] C. A. Balanis, Antenna Theory Analysis and Design Second Edition. New York, NY, USA: John Wiley and Sons, Inc., 1997.

[9] R. K. Mongia, A. Ittipiboon, and M. Cuhaci, "Measurement of radiation efficiency of dielectric resonator antennas," IEEE Microwave Guided Wave Lett., vol. 4, no. 3, pp. 80-82, Mar. 1994. 
[10] R. K. Mongia and A. Ittipiboon, "Theoretical and experimental investigations on rectangular dielectric resonator antennas," IEEE Trans. Antennas Propagat., vol. 45, no. 9, pp. 1348-1356, Sept. 1997.

[11] S. A. Long, M. W. McAllister, and L. C. Shen, "The resonant cylindrical dielectric cavity antenna," IEEE Trans. Antennas Propagat., vol. 31, no. 3, pp. 406-412, May 1983.

[12] A. W. Glisson, D. Kajfez, and J. James, "Evaluation of modes in dielectric resonators using a surface integral equation formulation," IEEE Trans. Microwave Theory Tech., vol. 31, no. 12, pp. 1023-1029, Dec. 1983.

[13] D. Kajfez and P. Guillon, Eds., Dielectric Resonators. Oxford, MS, USA: Vector Fields, 1990.

[14] H. Y. Lo, K. W. Leung, K. M. Luk, and E. K. N. Yung, "Low profile equilateral-triangular dielectric resonator antenna of very high permittivity," Electronics Letters, vol. 35, no. 25, pp. 2164-2166, Dec. 1999.

[15] H. Y. Lo and K. W. Leung, "Excitation of low-profile equilateraltriangular dielectric resonator antenna using a conducting conformal strip," Microwave and Optical Technology Letters, vol. 29, no. 5, pp. 317319, June 2001.

[16] K. W. Leung, K. M. Luk, and K. Y. A. Lai, "Input impedance of hemispherical dielectric resonator antenna," Electronics Letters, vol. 27, no. 24, pp. 2259-2260, Nov. 1991.

[17] E. A. J. Marcatili, "Dielectric rectangular waveguide and directional coupler for integrated optics," Bell Syst. Tech. J., vol. 48, pp. 2071-2132, Sept. 1969.

[18] K. M. Luk and K. W. Leung, Eds., Dielectric Resonator Antennas. Baldock, UK: Research Studies Press Ltd., 2003.

[19] Y. X. Guo, K. M. Luk, and K. W. Leung, "Mutual coupling between millimeter-wave dielectric resonator antennas," IEEE Trans. Microwave Theory Tech., vol. 47, no. 11, pp. 2164-2166, Nov. 1999.

[20] K. Y. Chow, K. W. Leung, K. M. Luk, and E. K. N. Yung, "Cylindrical dielectric resonator antenna array," Electronics Letters, vol. 31, no. 18, pp. 1536-1537, Aug. 1995. 
[21] B. Henry, A. Petosa, Y. M. M. Antar, and G. A. Morin, "Mutual coupling between multisegment dielectric resonator antennas," Microwave and Optical Technology Letters, vol. 21, no. 1, pp. 46-48, Apr. 1999.

[22] D. D. King, "Properties of dielectric image lines," IEEE Trans. Microwave Theory Tech., vol. 3, no. 2, pp. 75-81, Mar. 1955.

[23] R. M. Knox, "Dielectric waveguide microwave integrated circuits - an overview," IEEE Trans. Microwave Theory Tech., vol. 24, no. 11, pp. 806-814, Nov. 1976.

[24] K. Ogusu, "Numerical analysis of the rectangular dielectric waveguide and its modifications," IEEE Trans. Microwave Theory Tech., vol. 25, no. 11 , pp. $874-885$, Nov. 1977.

[25] R. T. Davis, "Millimeter-waves: controversy brews over transmission media," Microwaves, vol. 15, no. 3, pp. 32-42, Mar. 1976.

[26] T. Rozzi and M. Mongiardo, Open Electromagnetic Waveguides. London, UK: The Institute of Electrical Engineers, 1997.

[27] P. Toulios and R. Knox, "Rectangular dielectric image lines for milimeter integrated circuits," in Proc. of the IEEE Western Electronics Show and Convention, Los Angeles, CA, Aug. 1970, pp. 1-10.

[28] S. K. Koul, Millimeter Wave and Optical Dielectric Integrated Guides and Circuits. New York, NY, USA: John Wiley and Sons, Inc., 1997.

[29] K. Solbach and I. Wolff, "The electromagnetic fields and the phase constants of dielectric image lines," IEEE Trans. Microwave Theory Tech., vol. 26, no. 4, pp. 266-274, Apr. 1978.

[30] J. Xia, S. W. McKnight, and C. Vittoria, "Propagation losses in dielectric image guides," IEEE Trans. Microwave Theory Tech., vol. 36, no. 1, pp. 155-158, Jan. 1988.

[31] EMPIRE User and Reference Manual, 2003.

[32] K. Yee, "Numerical solution of inital boundary value problems involving Maxwell's equations in isotropic media," IEEE Trans. Antennas Propagat., vol. 14, no. 3, pp. 302-307, May 1966. 
[33] W. K. McRitchie and J. C. Beal, "Yagi-Uda array as a surface-wave launcher for dielectric image lines," IEEE Trans. Microwave Theory Tech., vol. 20, no. 8, pp. 493-496, Aug. 1972.

[34] T. N. Trinh, J. A. G. Malherbe, and R. Mittra, "A metal-to-dielectric waveguide transition with application to millimeter-wave integrated circuits," in IEEE MTT-S Int. Symp. Dig., vol. 80, no. 1, May 1980, pp. 205-207.

[35] J. E. Kietzer, A. R. Kaurs, and B. J. Levin, "A V-band communications transmitter and receiver system using dielectric waveguide integrated circuits," IEEE Trans. Microwave Theory Tech., vol. 24, no. 11, pp. 797-803, Nov. 1976.

[36] J. F. Miao and T. Itoh, "Coupling between microstrip line and image guide through small apertures in the common ground plane," IEEE Trans. Microwave Theory Tech., vol. 31, no. 4, pp. 361-363, Apr. 1983.

[37] Y. Shih, J. Rivera, and T. Itoh, "Directive planar excitation of an imageguide," in IEEE MTT-S Int. Symp. Dig., vol. 81, no. 1, June 1981, pp. $5-7$.

[38] K. Solbach, "Slots in dielectric image line as mode launchers and circuit elements," IEEE Trans. Microwave Theory Tech., vol. 29, no. 1, pp. 1016, Jan. 1981.

[39] I. J. Bahl and P. Bhartia, "Aperture coupling between dielectric image lines," IEEE Trans. Microwave Theory Tech., vol. 29, no. 9, pp. 891-896, Sept. 1981.

[40] K. Solbach, "Millimeter-wave dielectric image line detector-circuit employing etched slot structure," IEEE Trans. Microwave Theory Tech., vol. 29, no. 9, pp. 953-957, Sept. 1981.

[41] H. Tehrani, M. Y. Li, and K. Chang, "Broadband microstrip to dielectric image line transitions," IEEE Microwave Guided Wave Lett., vol. 10, no. 10 , pp. 409-411, Oct. 2000.

[42] H. Hammad, Y. M. M. Antar, A. Freundorfer, and S. Mahmoud, "Uniplanar CPW-fed slot launchers for efficient $\mathrm{TM}_{0}$ surface-wave excitation," IEEE Trans. Microwave Theory Tech., vol. 51, no. 4, pp. 1234-1240, Apr. 2003. 
[43] M. T. Birand, "Millimeter wave antenna array system," United Kingdom Patent GB2 097 196, Oct. 27, 1982.

[44] J. R. James and A. Henderson, "Dielectric image waveguide antenna array," U.S. Patent 4507664 , Mar. 26, 1985.

[45] F. Farzaneh, P. Guillon, and Y. Garault, "Coupling between a dielectric image guide and a dielectric resonator," in IEEE MTT-S Int. Symp. Dig., vol. 84, no. 1, May 1984, pp. 115-117.

[46] M. Wyville, A. Petosa, and J. S. Wight, "DIG feed for DRA arrays," in IEEE AP-S Int. Symp. Dig., vol. 2b, Washington, DC, July 2005, pp. $176-179$.

[47] S. E. Miller, "Coupled wave theory and waveguide applications," Bell Syst. Tech J., vol. 33, no. 3, pp. 661-719, May 1954.

[48] A. Villeneuve, "Taylor patterns for discrete arrays," IEEE Trans. Antennas Propagat., vol. 32, no. 10, pp. 1089-1093, Oct. 1984.

[49] A. Petosa, R. K. Mongia, A. Ittipiboon, and J. S. Wight, "Design of microstrip-fed series array of dielectric resonator antennas," Electronics Letters, vol. 31, no. 16, pp. 1306-1307, Aug. 1995.

[50] C. Nguyen, Analysis Methods for RF, Microwave and Millimeter-Wave Planar Transmission Line Structures. New York, NY, USA: John Wiley and Sons, 2000.

[51] P. K. Ikalainen and G. L. Matthaei, "Design of broad-band dielectric waveguide 3-dB couplers," IEEE Trans. Microwave Theory Tech., vol. 35, no. 7, pp. 621-628, July 1987.

[52] M. T. K. Tam and R. D. Murch, "Half volume dielectric resonator antenna designs," Electronics Letters, vol. 33, no. 23, pp. 1914-1916, Nov. 1997. 